\title{
Otimização Global e o Problema de Designação de Fluxos e Capacidades
}

\author{
Marcelo Gomes de Queiroz
}

\author{
DISSERTAÇÃO DE MESTRADO APRESENTADA AO \\ INSTITUTO DE MATEMÁTICA E ESTATÍSTICA \\ DA \\ UNIVERSIDADE DE SÃO PAULO
}

Curso: Mestrado em Matemática Aplicada Área de Concentração: Ciência da Computação

Orientador: Prof. Dr. Carlos Humes Júnior

São Paulo, 1997

O aluno recebeu apoio da CAPES. 



\section{Otimização Global e o Problema de Designação de Fluxos e Capacidades}

Este exemplar corresponde à redação final da dissertação apresentada por Marcelo Gomes de Queiroz, devidamente corrigida e aprovada pela comissão julgadora.

São Paulo, setembro de 1997.

Banca examinadora:

Prof. Dr. Carlos Humes Júnior

IME - USP

Prof. Dr. Arnaldo Mandel

IME - USP

Prof. Dr. Nelson Maculan

COPPE - UFRJ 


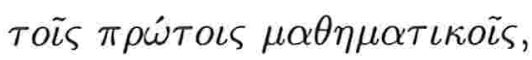
oí $\mu \varepsilon \tau \grave{\alpha} \kappa \alpha \lambda o \tilde{v} \gamma \rho \alpha ́ \alpha \mu \alpha \tau o \varsigma$

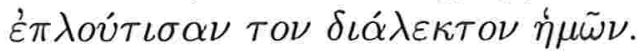


Aos primeiros matemáticos, que com suas belas letras enriqueceram nossa linguagem. 


\section{Agradecimentos}

Aos meus pais, por tudo.

Ao Carlos, pela constante dedicação, pelo modelo de trabalho e, mais ainda, pela compreensão e paciência que só poderiam vir de um amigo.

Aos amigos matemáticos, Paulo J. da S. e Silva, Dilma M. Silva e Marcelo D. Passos; e aos amigos não-matemáticos, Roberto de L. Rodrigues, Cláudia S. Gentil e Luis C. G. Oliva, por tudo o que não diz respeito a esta dissertação.

Mais ainda, a todos os outros amigos, cujos nomes aqui não cabem, pela futura compreensão em relação à presente omissão.

Aos professores Arnaldo Mandel, Carlos E. Ferreira, José Coelho de Pina, Nelson

Maculan e Yoshiko Wakabayashi, pelas importantes discussões e sugestões.

Aos professores P. R. Kumar e R. T. Rockafellar, pela inestimável oportunidade de discussão a respeito deste trabalho.

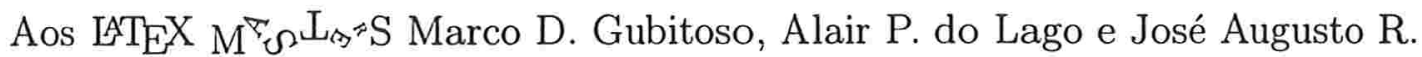
Soares, pelas várias ajudas com este programa. 



\title{
Sumário
}

Neste trabalho, é estudado o Problema de Designação de Fluxos e Capacidades em redes de computadores, sob a perspectiva de Programação Côncava. É obtida uma condição necessária para a caracterização de vértices do poliedro de fluxos multicomodidade, bem como um algorítmo para o cômputo das arestas a partir de um vértice. A partir destas ferramentas, são propostas duas heurísticas para a busca do mínimo global do problema, cujas implementações e testes computacionais são incluídos, e comparados à aplicação do algorítmo de Tŭ-Zwart a este problema.

\begin{abstract}
This work deals with the Capacity and Flow Assignment Problem for computer networks, viewed as a Concave Programming problem. A necessary condition for the characterization of extremal points of the multicommodity flow polyhedron is attained, as well as an algorithm for the computation of the edges leaving a given extremal point. With these tools, two heuristics for the search of a global minimum for the problem are proposed. Their implementation and computational tests are also included, and compared to the application of the Tuй-Zwart algorithm to this problem.
\end{abstract}





\section{Sumário}

Lista de Definições r xi

Lista de Teoremas, Proposições e Corolários xiii

Lista de Algorítmos e Heurísticas $\quad$ xv

Notações

Introdução

1 Conceitos Básicos $\quad 1$

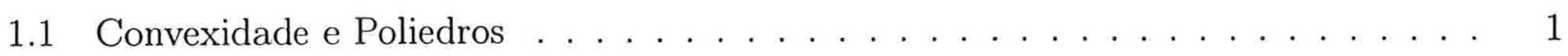

1.1 .1 O Poliedro Canônico . . . . . . . . . . . . . . . . . . . 3

1.1.2 Transformações de Poliedros . . . . . . . . . . . . . . . . . 6

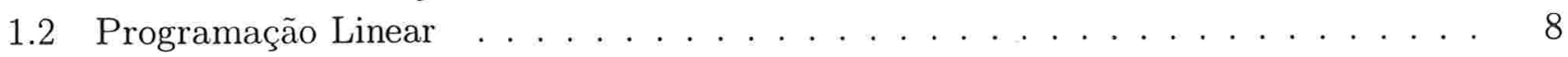

1.2.1 Dualidade em Programação Linear . . . . . . . . . . . . . . . . 9

1.2 .2 Decomposição de Dantzig-Wolfe . . . . . . . . . . . . . . . . . . . . . . . . . . . . .

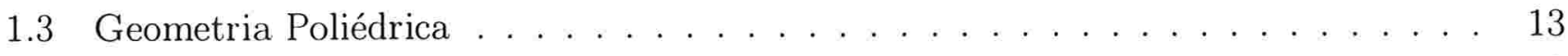

1.4 Grafos e Fluxos . . . . . . . . . . . . . . . . . . . . . . . . . . . . 18

2 O Problema Motivador $\quad 23$

2.1 A Rede LARC . . . . . . . . . . . . . . . . . . . . . . . . . . . . 23

2.2 O $(\mathrm{CFA})$ e suas Formulações . . . . . . . . . . . . . . . . . . . . . . . . . . . . . . . . . . . . . . . . . . .

2.2 .1 Descrição Informal . . . . . . . . . . . . . . . . . . . . 25

2.2 .2 Modelagem do Problema . . . . . . . . . . . . . . . . . 26

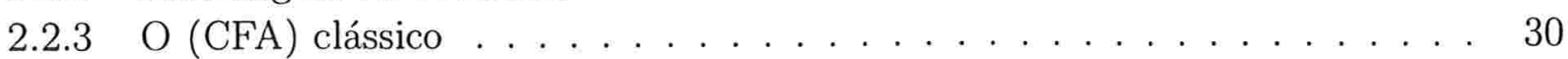

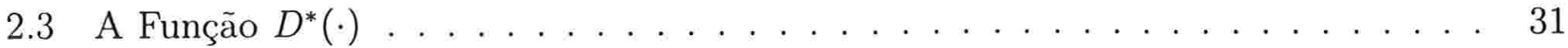

2.3.1 Técnica de linearizações externas para o cálculo de $D^{*}(\cdot) \ldots \ldots$. . . . . 38

2.4 Os Algoritmos de Tŭ́-Zwart e Falk-Hoffman . . . . . . . . . . . . . . . . . . . . 39

2.4 Tŭ-Zwart . . . . . . . . . . . . . . . . . . . . . . . 40

2.4 .2 Falk-Hoffman . . . . . . . . . . . . . . . . . . . . . . . . . . . . . . 42

2.5 Problemas de Aplicabilidade de Tŭ-Zwart ao (CFA) . . . . . . . . . . . . . . 45 
3 Estudo do Poliedro de Fluxos Multicomodidade $\quad 47$

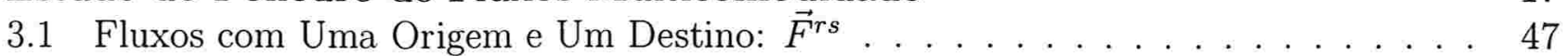

3.1 .1 Descrição de $F^{r s}$ por Cortes . . . . . . . . . . . . . . . . . . . . . . 49

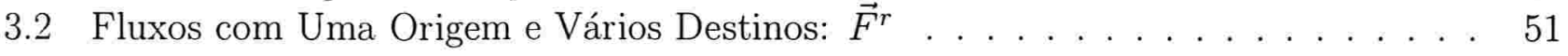

3.2 .1 Descrição de $F^{r}$ por Cortes . . . . . . . . . . . . . . . . . . . 53

3.3 Fluxos com Várias Origens e Destinos: $\overrightarrow{\mathcal{F}}$ e $\mathcal{F} \ldots \ldots \ldots$

3.3.1 Cômputo das arestas em $\mathcal{F} \ldots \ldots \ldots \ldots$. . . . . . . . . . . 65

3.3.2 Descrição de $\mathcal{F}$ por Cortes . . . . . . . . . . . . . . . . . . . . 72

4 Algorítmos e Heurísticas $\quad 77$

4.1 Heurísticas Baseadas em Tŭ-Zwart . . . . . . . . . . . . . . . . . . 77

4.2 Tŭ-Zwart Aplicado a $\vec{F}^{1} \times \vec{F}^{2} \times \ldots \times \vec{F}^{n} \ldots \ldots \ldots \ldots$

4.3 Resultados Computacionais e Análise Comparativa . . . . . . . . . . . . . . . 88

5 Conclusões e Trabalho Futuro $\quad 99$ 


\section{Lista de Definições}

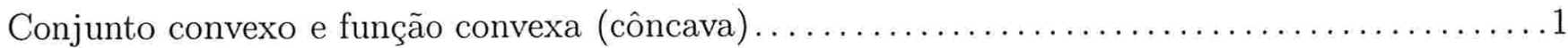

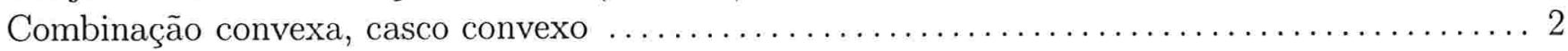

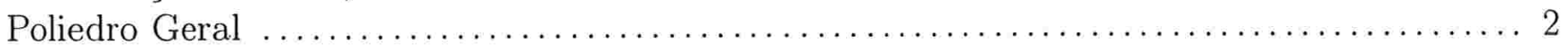

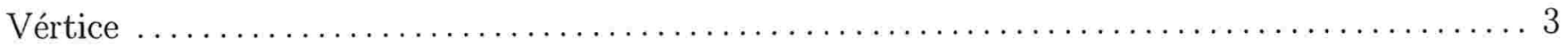

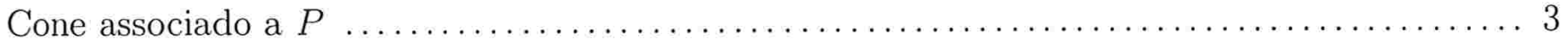

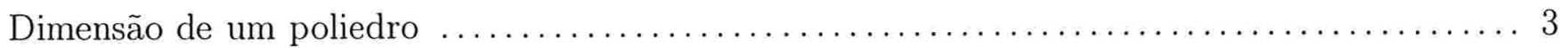

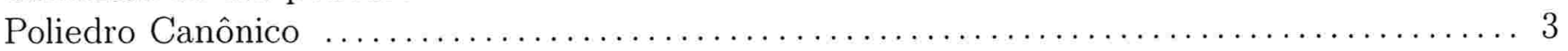

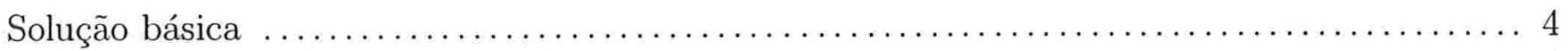

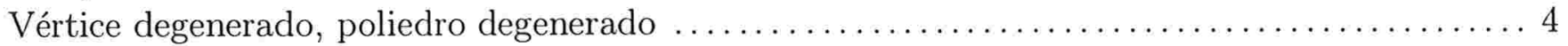

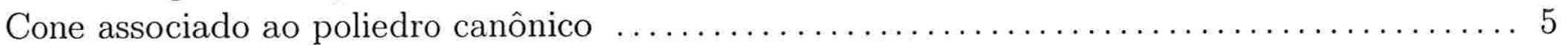

O problema canônico de programação linear $\ldots \ldots \ldots \ldots \ldots \ldots \ldots \ldots \ldots \ldots \ldots \ldots \ldots \ldots \ldots$

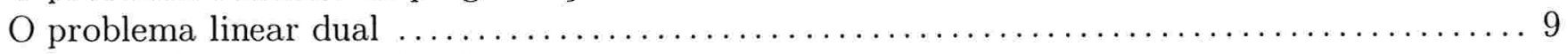

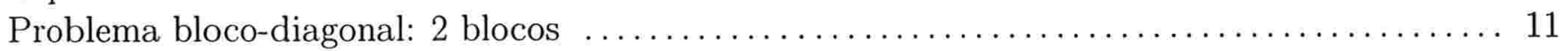

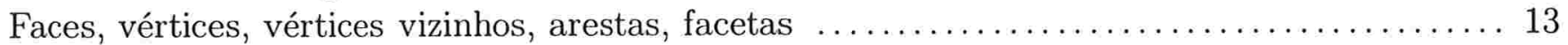

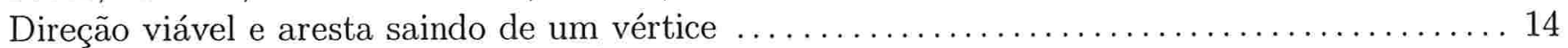

Grafos, Caminhos, Ciclos, Cortes e Árvores $\ldots \ldots \ldots \ldots \ldots \ldots \ldots \ldots \ldots \ldots \ldots \ldots \ldots \ldots \ldots \ldots$

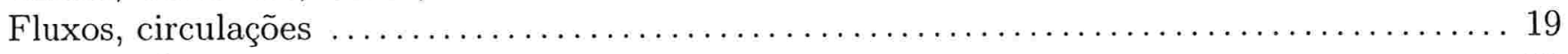

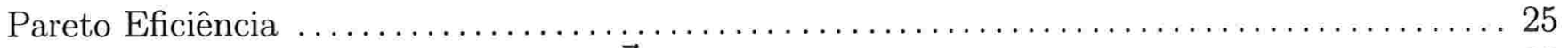

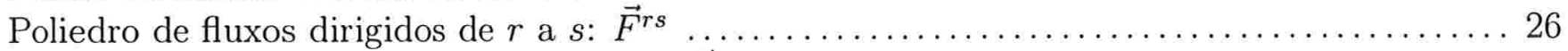

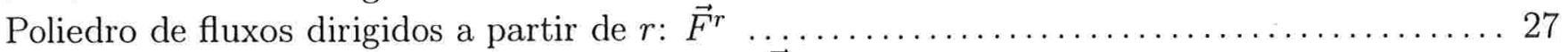

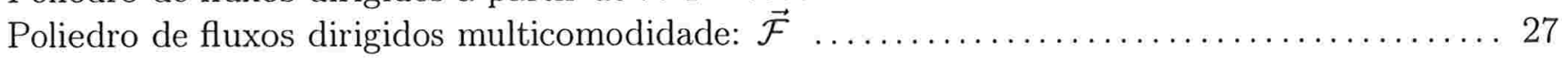

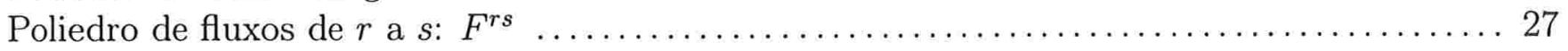

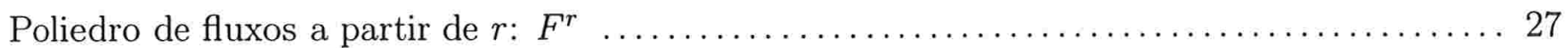

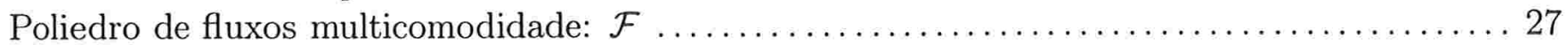

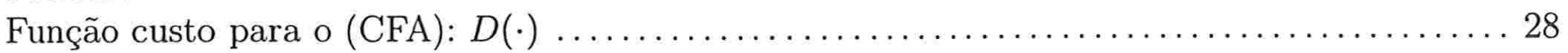

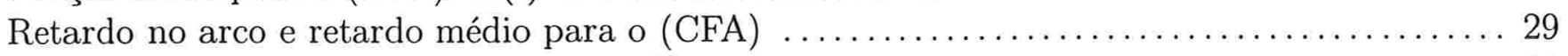

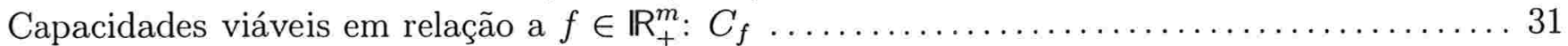

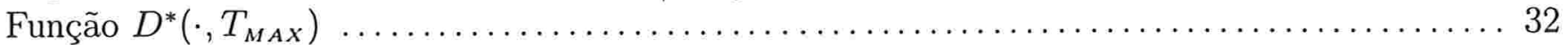

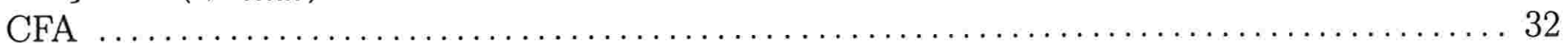

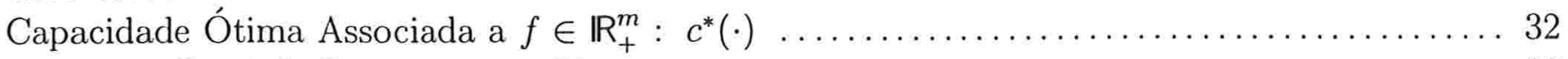

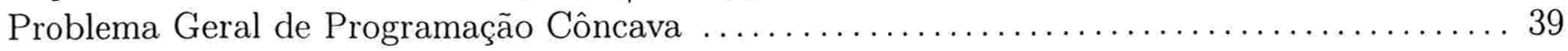

Vértices mínimos locais em relação aos seus vizinhos $\ldots \ldots \ldots \ldots \ldots \ldots \ldots \ldots \ldots \ldots \ldots \ldots . \ldots \ldots$

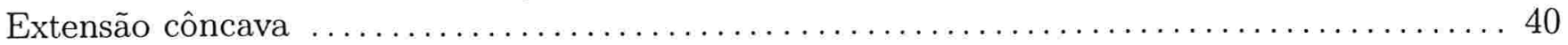

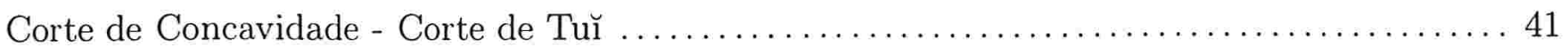

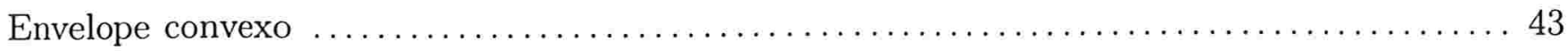




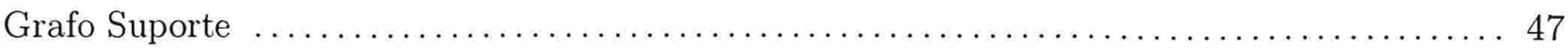

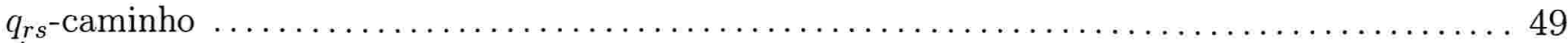

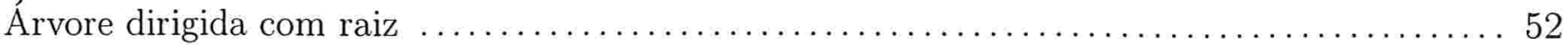

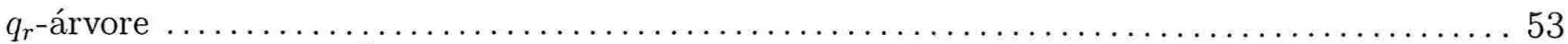

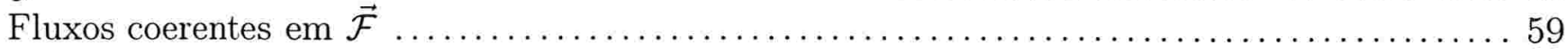

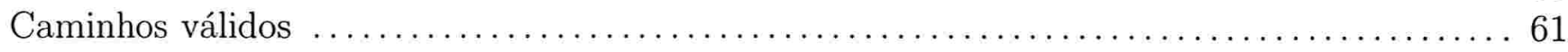

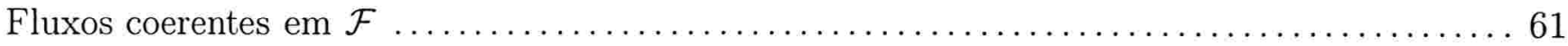

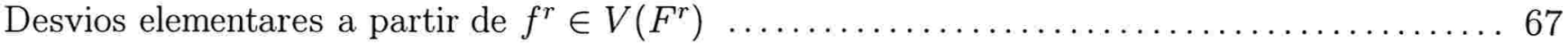

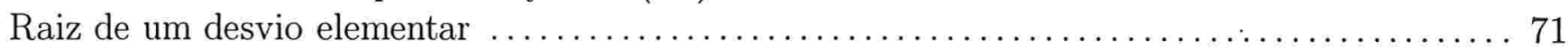

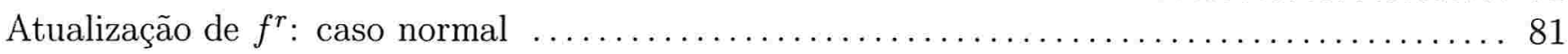

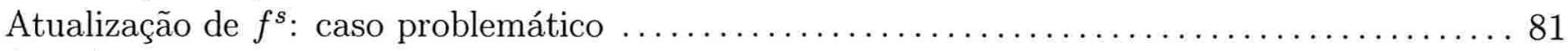

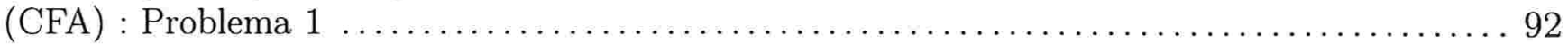

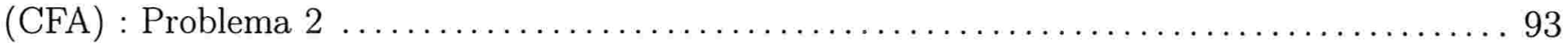

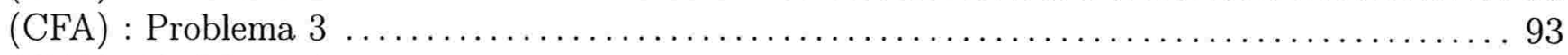

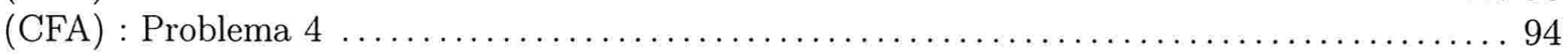

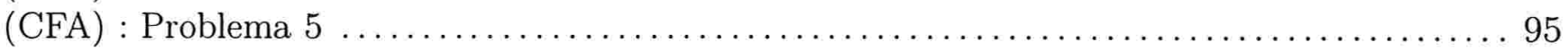

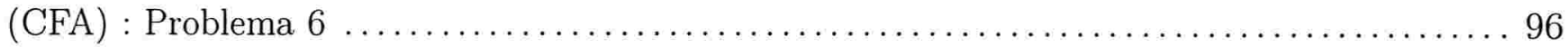

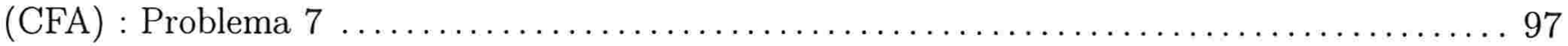




\section{Lista de Teoremas, Proposições e Corolários}

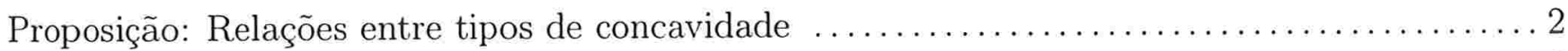

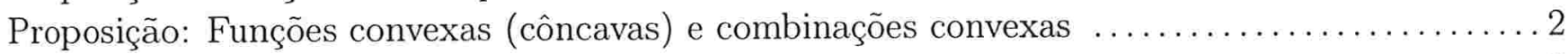

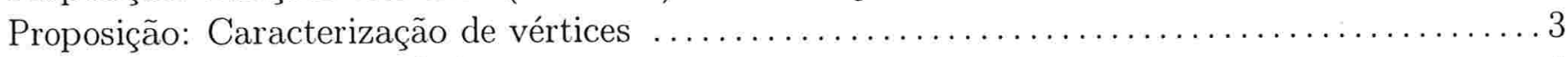

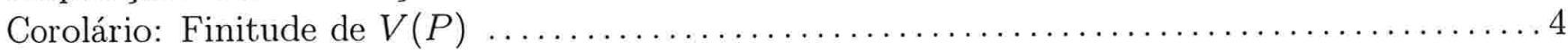

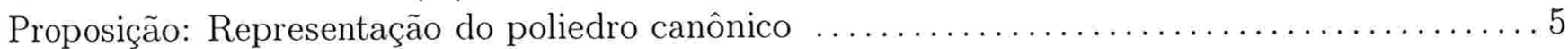

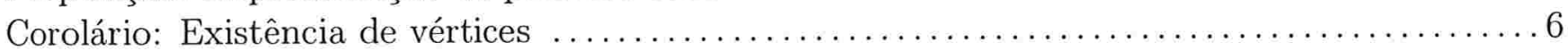

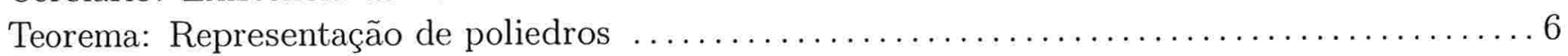

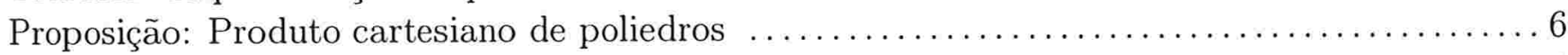

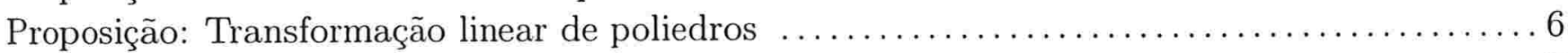

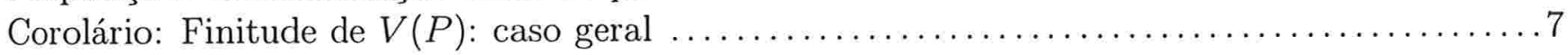

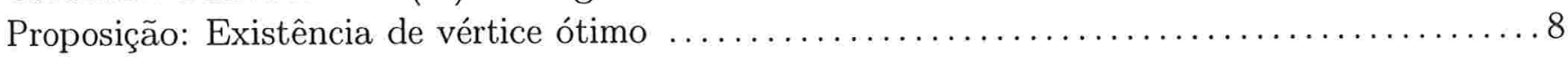

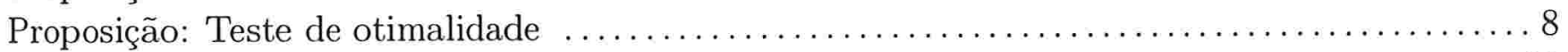

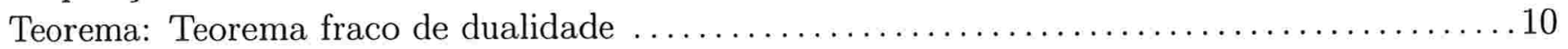

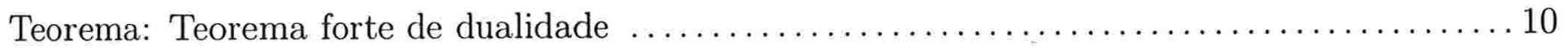

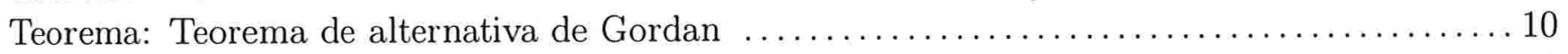

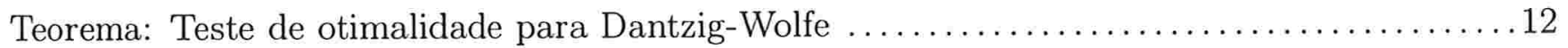

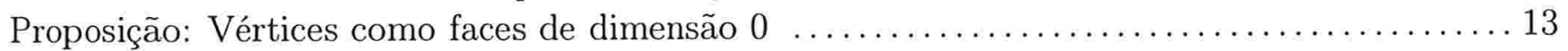

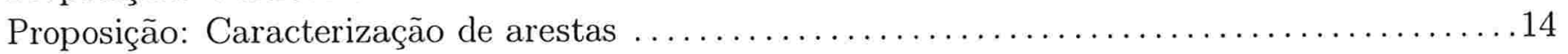

Proposição: Número de arestas saindo de $v$ : poliedro canônico $\ldots \ldots \ldots \ldots \ldots \ldots \ldots \ldots \ldots \ldots$

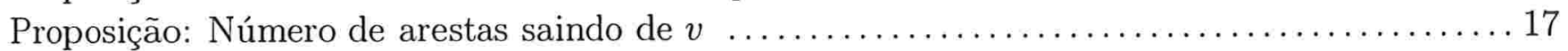

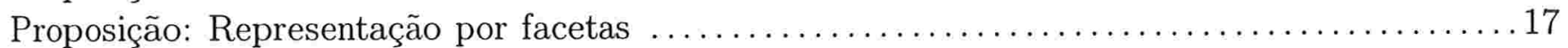

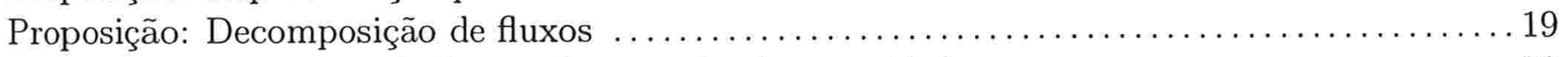

Proposição: Existência de fluxo sob restrição de capacidade nos $\operatorname{arcos} \ldots \ldots \ldots \ldots \ldots \ldots \ldots 20$

Proposição: Expressão de $c^{*}(\cdot)$ e $D^{*}(\cdot)$ para o caso de custos lineares $\ldots \ldots \ldots \ldots \ldots \ldots \ldots \ldots 33$

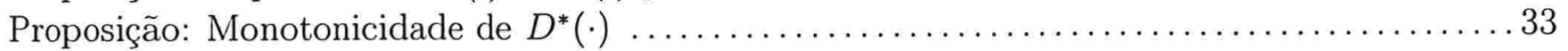

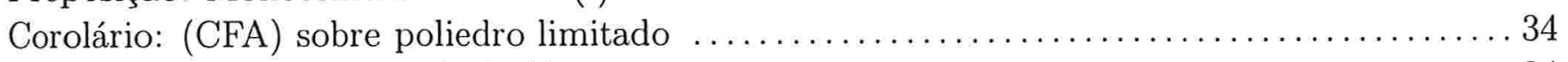

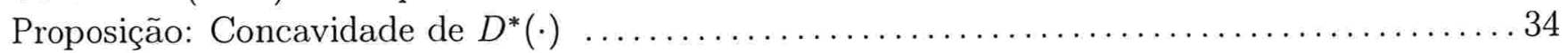

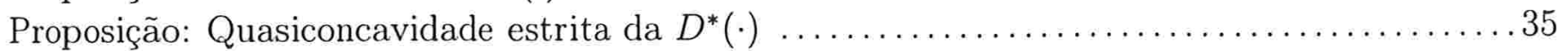

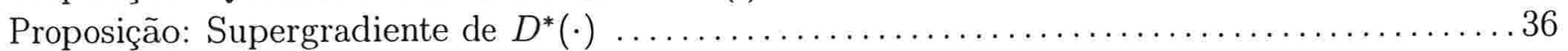

Proposição: Condição necessária de otimalidade para programação côncava $\ldots \ldots \ldots \ldots \ldots \ldots 43$

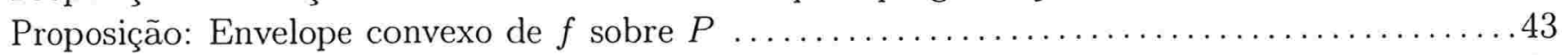

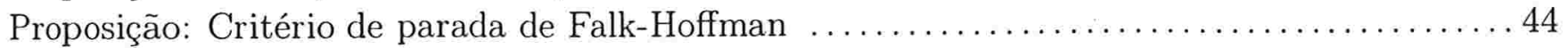


Proposição: Representação canônica de $\vec{F}^{r s}$

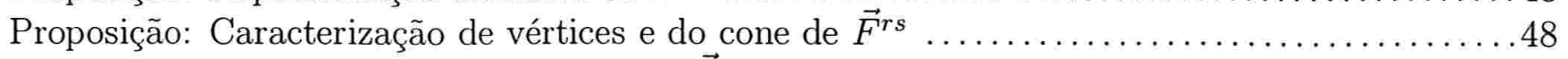

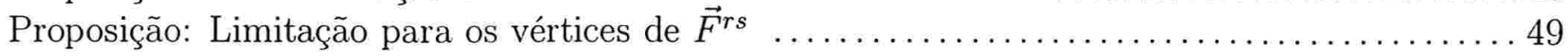

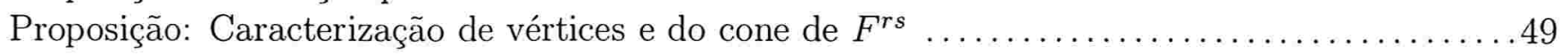

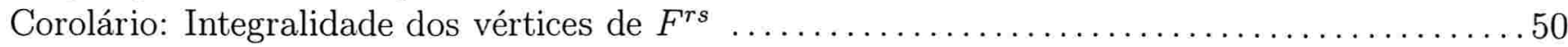

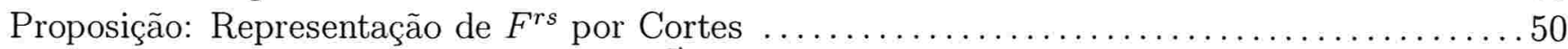

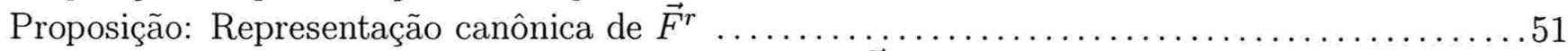

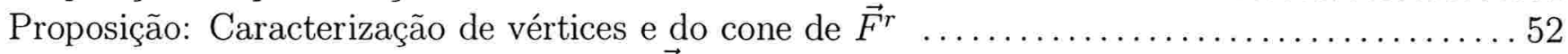

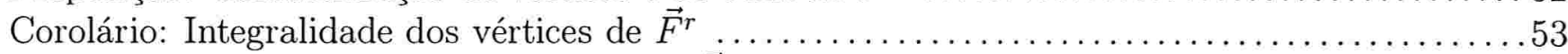

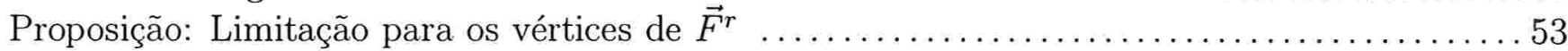

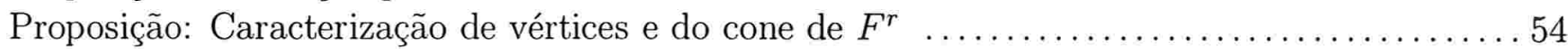

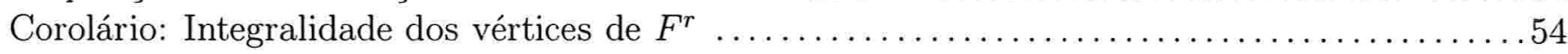

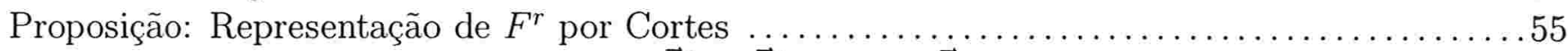

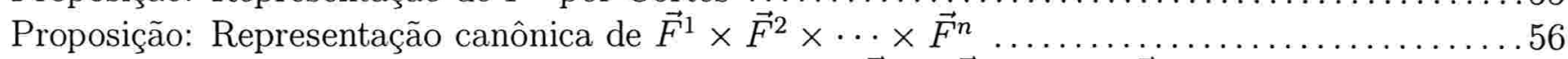

Proposição: Caracterização de vértices e do cone de $\vec{F}^{1} \times \vec{F}^{2} \times \ldots \times \vec{F}^{n} \ldots \ldots \ldots \ldots \ldots \ldots \ldots 6$

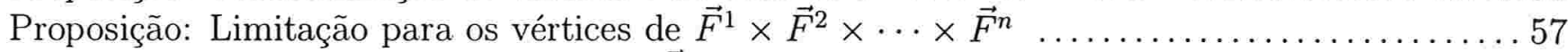

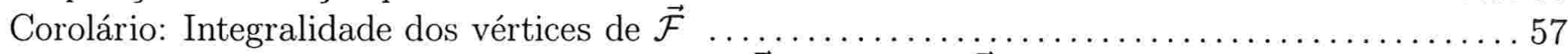

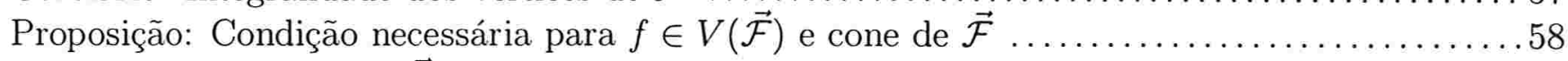

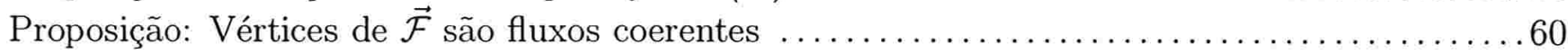

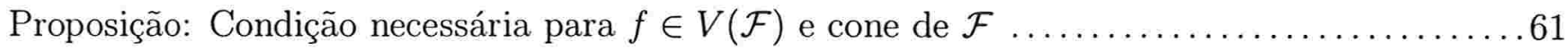

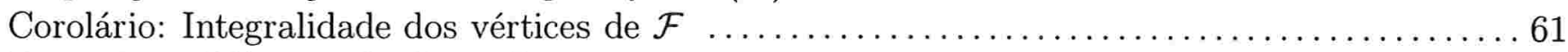

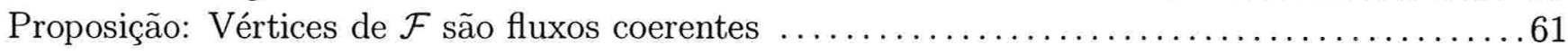

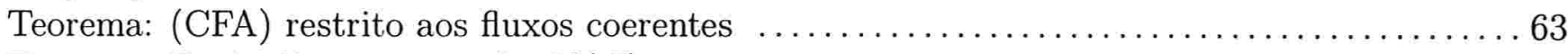

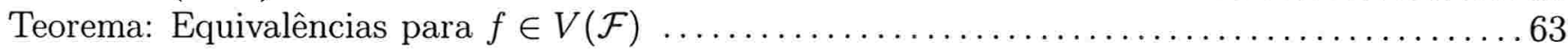

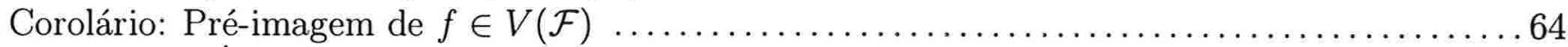

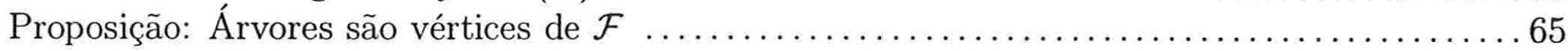

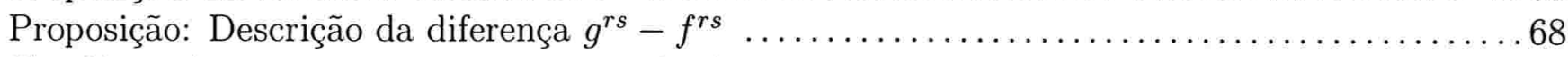

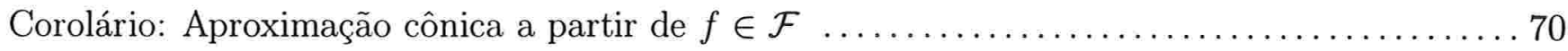

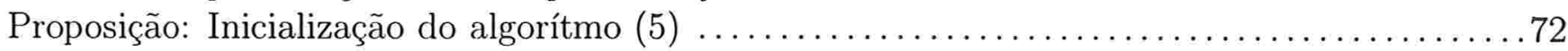

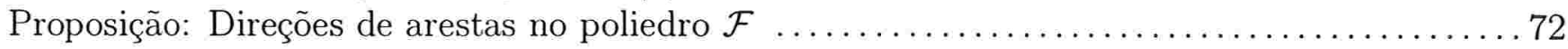

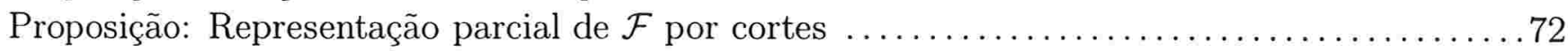

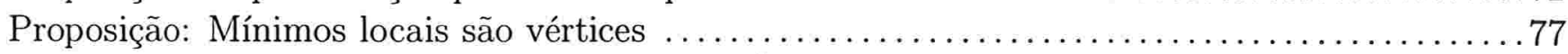

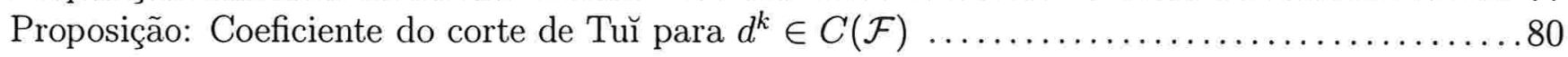




\section{Lista de Algorítmos e Heurísticas}

Algorítmo: Método inocente para o cômputo de arestas $\ldots \ldots \ldots \ldots \ldots \ldots \ldots \ldots \ldots \ldots \ldots \ldots 15$

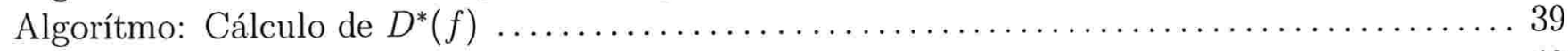

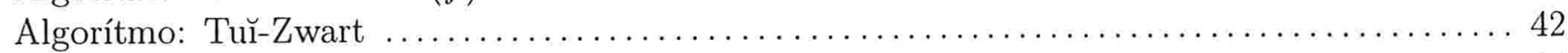

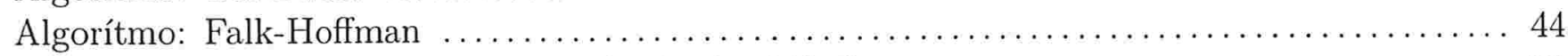

Algorítmo: Cômputo de arestas saindo de $f \in V(\mathcal{F}) \ldots \ldots \ldots \ldots \ldots \ldots \ldots \ldots \ldots \ldots \ldots \ldots \ldots \ldots \ldots$

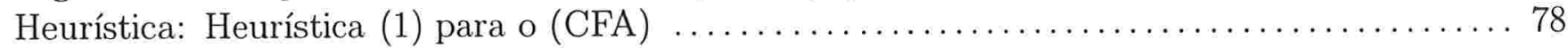

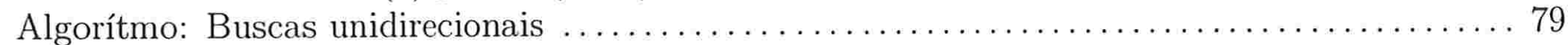

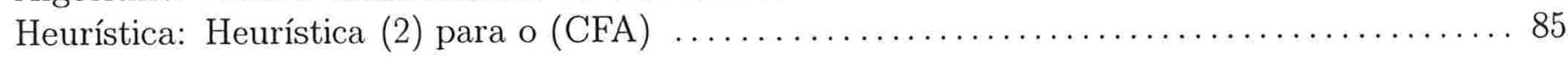




\section{Notações}

Notação 1 (Índices sobre-/sub-escritos) Se $x \in \mathbb{R}^{n}$, denotamos suas coordenadas por $x_{1}, x_{2}, \ldots, x_{n}$, ou $x=\left[\begin{array}{c}x_{1} \\ x_{2} \\ \vdots \\ x_{n}\end{array}\right]$. Todos os vetores são colunas.

Se $A \in M_{m \times n}(\mathbb{R})$ é uma matriz $m \times n$, denotamos por $A^{j}$ a j-ésima coluna de $A$, e por $A_{i}$ a i-ésima linha de $A$ :

$$
A=\left[\begin{array}{ccc}
\ddots & \vdots & \\
\cdots & a_{i j} & \cdots \\
& \vdots & \ddots
\end{array}\right]=\left[A^{1}\left|A^{2}\right| \cdots \mid A^{n}\right]=\left[\begin{array}{c}
\frac{A_{1}^{t}}{A_{2}^{t}} \\
\frac{\vdots}{A_{m}^{t}}
\end{array}\right] .
$$

Se I é um conjunto de indices, indicamos por $x_{I}=\left(x_{i}\right)_{i \in I}$ e $A_{I}=\left(A^{i}\right)_{i \in I}$. (A notação $A^{I}$, embora mais coerente, é menos freqüente na literatura).

Notação 2 (Conjunto de índices de componentes não-nulas de $x \in \mathbb{R}^{n}$ ) Denotamos por $I(x)$ o conjunto $\left\{i \in\{1,2, \ldots, n\} \mid x_{i} \neq 0\right\}$.

Notação 3 (Relações de ordem) Sejam $x \in \mathbb{R}^{n}, y \in \mathbb{R}^{n}$, e seja $\geq_{\mathbf{R}}$ a relação de ordem usual em $\mathbb{R}$. Definimos:

$$
\begin{aligned}
& x \geqq y \quad \Longleftrightarrow x_{i} \geq_{\mathbf{R}} y_{i}, \text { para } i=1,2, \ldots, n ; \\
& x \geq y \quad \Longleftrightarrow x_{i} \geq_{\mathbf{R}} y_{i}, \text { para } i=1,2, \ldots, n, \text { e } x \neq y ; \\
& x>y \Longleftrightarrow x_{i}>_{\mathbf{R}} y_{i}, \text { para } i=1,2, \ldots, n .
\end{aligned}
$$

Notação 4 (Funções) Seja $f: X \longrightarrow Y, e A \subset X$. Denotamos por $f(A)$ o conjunto

$$
f(A)=\{y \in Y \mid \exists x \in A: y=f(x)\} .
$$

Se $X$ é finito, usaremos a notação $f_{i}=f(i)$. 
xviii

Notação 5 (Bola aberta e interior) Denotamos a bola aberta de centro $\bar{x}$ e raio $\varepsilon$ por $B(\bar{x}, \varepsilon)=\left\{x \in \mathbb{R}^{n} \mid\|x-\bar{x}\|<\varepsilon\right\}$.

Se $A \subset \mathbb{R}^{n}$ denotamos o seu interior por $\operatorname{Int}(A)$ onde

$$
x \in \operatorname{Int}(A) \Longleftrightarrow \exists U \text { aberto t.q. } x \in U \subset A \text {. }
$$

Notação 6 (Soma de conjuntos) $S e A, B \subset \mathbb{R}^{n}$, denotamos por

$$
A+B=\{a+b \mid a \in A, b \in B\} .
$$

Notação 7 (Hiperplanos, semi-espaços) Para simplificar alguns pontos da apresentação, utilizaremos a notação $\{\langle a, x\rangle \leqq b\}=\left\{x \in \mathbb{R}^{n} \mid\langle a, x\rangle \leqq b\right\}$. Analogamente definimos $\{\langle a, x\rangle \geqq b\} \quad e$ $\{\langle a, x\rangle=b\}$

Notação 8 (Reais estendidos) Denotamos por $\overline{\mathbb{R}}$ o conjunto dos reais estendidos, isto é,

$$
\overline{\mathbb{R}}=\mathbb{R} \cup\{+\infty,-\infty\}
$$




\section{Introdução}

Neste trabalho, apresentamos o problema de Designação de Fluxos e Capacidades em redes de computadores, e procuramos enfatizar a potencial interação entre este problema e uma grande variedade de áreas de estudo, como Programação Linear e Não-Linear, Análise Convexa, Geometria Poliédrica (também conhecida como Combinatória Poliédrica), Teoria dos Grafos, Otimização Global e em particular Programação Côncava, para citar algumas.

Nosso principal ponto de partida é o trabalho desenvolvido por [Humes 88], que descreveu o (CFA) - Capacity and Flow Assignment - como um problema de programação côncava, isto é, como um problema de minimizar uma função côncava sobre um poliedro. Problemas desta natureza podem possuir muitos mínimos locais que não são globais, e condições locais (como, por exemplo, as baseadas em gradientes) não são suficientes para estabelecer a otimalidade (global) de um ponto. Em contrapartida, estes problemas tem a propriedade de que se existe alguma solução ótima, então existe um vértice ótimo.

O (CFA) tem porém algumas peculiaridades em relação a outros problemas de programação côncava. Em tom quase que jocoso, diríamos que no (CFA) estamos interessados em minimizar uma função côncava para a qual não conhecemos a fórmula, sobre um poliedro para o qual não conhecemos a descrição. Na realidade, o cálculo da função côncava em um ponto do poliedro corresponde a resolver um problema de programação não-linear; e o poliedro, imerso em um espaço de dimensão dada pelo número de canais na rede $(m)$, é dado por uma "projeção" de um outro poliedro, imerso em um espaço de dimensão $2 m n$, onde $n$ é o número de nós da rede.

Dentro deste contexto, apresentamos duas heurísticas para o (CFA) na dimensão "baixa" e um algoritmo para o (CFA) na dimensão "alta", juntamente com os resultados teóricos que permitiram a idealização e realização destes métodos. Outros resultados teóricos foram também incluidos, como subsídio para futuros tópicos interessantes de pesquisa.

$\mathrm{Na}$ fase de redação deste texto duas forças, de certo modo conflitantes, estiveram presentes.

A primeira delas era a tentativa de criar um texto claro e objetivo e, na medida do possível, auto-contido. Tendo como típico leitor-alvo um aluno de iniciação científica, consideramos razoável incluir um capítulo dedicado aos pré-requisitos deste trabalho, capítulo este que deveria apresentar as principais definições e resultados que seriam utilizados no restante do texto, bem como introduzir notações e nomenclaturas convenientes. Em suma, um capítulo de referência.

A outra força envolvida era a necessidade de transmitir o processo da pesquisa, que é por excelência um processo dinâmico, sempre apontando para novas direções. Alguns resultados novos do capítulo 2 utilizam em suas demonstrações noções básicas de programação não-linear (como por exemplo, condições de Kuhn-Tucker e condições de qualificação), que não foram incluidas no capítulo 1. Vários resultados do capítulo 3 foram incluidos por serem interessantes em sí, mas não foram ainda aplicados ao problema; citamos, por exemplo, a integralidade dos vértices do poliedro 
de fluxos multicomodidade (no caso das demandas serem todas inteiras), ou ainda a descrição de um subconjunto das facetas deste mesmo poliedro.

Com isto em mente, organizamos o texto assim:

Precedendo o primeiro capítulo, incluimos uma lista de notações que serão utilizadas em todo o texto. Recomendamos fortemente a leitura desta lista, pois algumas notações não são standard.

O primeiro capítulo inclui noções básicas de convexidade, poliedros, programação linear, geometria poliédrica, grafos e fluxos. Longe de pretender ensinar todos estes tópicos em 20 páginas, o primeiro capítulo pode ser usado como uma breve revisão e principalmente como referência para os principais resultados utilizados neste trabalho.

O capítulo 2 apresenta o problema motivador da dissertação. Entendemos por problema motivador não somente o problema real de designação de fluxos e capacidades que foi a origem do trabalho desenvolvido em [Humes 88], mas também as ferramentas e resultados teóricos que motivaram a direção de pesquisa que tomamos.

No terceiro capítulo estudamos vários poliedros de fluxo, de maneira hierárquica (e esperamos, didática), enquanto etapas na "construção" do poliedro de fluxos multicomodidade.

O quarto capítulo ocupa-se de agregar todos os materiais teóricos dos capitulos 2 e 3, apresentando duas heurísticas e um algoritmo para o (CFA), com detalhes de implementação e uma breve análise dos resultados computacionais obtidos.

O último capítulo é na verdade um epílogo, indicando tópicos interessantes de pesquisa a partir dos resultados e exemplos aqui compilados.

As referências encontradas no final do texto compreendem não só textos que tiveram uma aplicação imediata neste trabalho, mas também textos relacionados com a pesquisa aqui apresentada e que serão de serventia para qualquer pessoa que se interessar pelo conteúdo desta dissertação.

Todos os códigos referentes às heurísticas e ao algorítmo podem ser obtidos por ftp anônimo em ftp://ftp.ime.usp.br/pub/mqz/ 


\section{Capítulo 1}

\section{Conceitos Básicos}

Neste capítulo estaremos apresentando o material básico que utilizaremos no restante deste trabalho. Faremos referência a vários textos básicos, cujo material procuramos apresentar aqui fazendo uso de uma notação unificada. Pretendemos que este seja um capítulo de referência, cuja leitura poderá ser omitida ou adiada convenientemente.

\subsection{Convexidade e Poliedros}

Definição 1.1 (Conjunto convexo e função convexa (côncava)) Dizemos que $A \subset \mathbb{R}^{n}$ é convexo se para quaisquer $x^{1}, x^{2} \in A$ e $\lambda \in[0,1]$ temos $\lambda x^{1}+(1-\lambda) x^{2} \in A$.

Seja $X$ um conjunto convexo:

Dizemos que $f: X \longrightarrow \mathbb{R}$ é convexa $s e \forall x^{1}, x^{2} \in X, \forall \lambda \in(0,1)$,

$$
f\left(\lambda x^{1}+(1-\lambda) x^{2}\right) \leqq \lambda f\left(x^{1}\right)+(1-\lambda) f\left(x^{2}\right)
$$

$f$ é côncava se $(-f)$ é convexa, isto é, se $\forall x^{1}, x^{2} \in X, \forall \lambda \in(0,1)$,

$$
f\left(\lambda x^{1}+(1-\lambda) x^{2}\right) \geqq \lambda f\left(x^{1}\right)+(1-\lambda) f\left(x^{2}\right) .
$$

$f$ é estritamente côncava (estritamente convexa) se é côncava (convexa) e a desigualdade correspondente é obedecida estritamente, $\forall x^{1}, x^{2} \in X: x^{1} \neq x^{2}, \forall \lambda \in(0,1)$.

$f$ é quasicôncava se $\forall x^{1}, x^{2} \in X, \forall \lambda \in(0,1)$,

$$
f\left(\lambda x^{1}+(1-\lambda) x^{2}\right) \geqq \min \left\{f\left(x^{1}\right), f\left(x^{2}\right)\right\} .
$$

$f$ é estritamente quasicôncava se a última desigualdade é obedecida estritamente, $\forall x^{1}, x^{2} \in X: x^{1} \neq x^{2}, \forall \lambda \in(0,1)$.

[Mangasarian 69] fornece caracterizações interessantes de convexidade (concavidade) para funções diferenciáveis. Além disto apresenta algumas relações de verificação imediata: 
Proposição 1.2 (Relações entre tipos de concavidade) Seja $f: X \longrightarrow \mathbb{R}^{n}$. Então

$$
\begin{aligned}
f \text { é estritamente côncava } & \Longrightarrow f \text { é côncava } \\
f \text { é estritamente quasicôncava } & \Longrightarrow f \text { é quasicôncava } \\
f \text { é côncava } & \Longrightarrow f \text { é quasicôncava } \\
f \text { é estritamente côncava } & \Longrightarrow f \text { é estritamente quasicôncava }
\end{aligned}
$$

Definição 1.3 (Combinação convexa, casco convexo) Uma combinação convexa dos elementos $x^{1}, x^{2}, \ldots, x^{k} \in \mathbb{R}^{n}$ é um elemento da forma

$$
\sum_{i=1}^{k} \alpha_{i} x^{i}
$$

onde $\alpha \geqq 0$ e $\sum_{i=1}^{k} \alpha_{i}=1$.

$O$ casco convexo de um conjunto $X \subset \mathbb{R}^{n}$, denotado por $[X]$, é definido por

$$
[X]=\left\{y \in \mathbb{R}^{n} \mid y=\sum_{i=1}^{k} \alpha_{i} x^{i}, \alpha \geqq 0, \sum_{i=1}^{k} \alpha_{i}=1 \text { e } x^{i} \in X, i=1, \ldots, k\right\} .
$$

Segue imediatamente desta definição:

Proposição 1.4 (Funções convexas (côncavas) e combinações convexas) Sejam $X \subset \mathbb{R}^{n}, x^{1}, \ldots, x^{k} \subset X$, e $\alpha \geqq 0, \sum_{i=1}^{k} \alpha_{i}=1$, e $f: X \longrightarrow \mathbb{R}$, então:

$$
\begin{aligned}
f \text { é convexa } & \Longrightarrow f\left(\sum_{i=1}^{k} \alpha_{i} x^{i}\right) \leqq \sum_{i=1}^{k} \alpha_{i} f\left(x^{i}\right) ; \\
f \text { é côncava } & \Longrightarrow f\left(\sum_{i=1}^{k} \alpha_{i} x^{i}\right) \geqq \sum_{i=1}^{k} \alpha_{i} f\left(x^{i}\right) ; \\
f \text { é estritamente convexa } & \Longrightarrow f\left(\sum_{i=1}^{k} \alpha_{i} x^{i}\right)<\sum_{i=1}^{k} \alpha_{i} f\left(x^{i}\right) ; \\
f \text { é estritamente côncava } & \Longrightarrow f\left(\sum_{i=1}^{k} \alpha_{i} x^{i}\right)>\sum_{i=1}^{k} \alpha_{i} f\left(x^{i}\right) ; \\
f \text { é quasicôncava } & \Longrightarrow f\left(\sum_{i=1}^{k} \alpha_{i} x^{i}\right) \geqq \min \left\{f\left(x^{i}\right): i=1, \ldots, k\right\} ; \\
f \text { é estritamente quasicôncava } & \Longrightarrow f\left(\sum_{i=1}^{k} \alpha_{i} x^{i}\right)>\min \left\{f\left(x^{i}\right): i=1, \ldots, k\right\} .
\end{aligned}
$$

Os conceitos de hiperplano (semi-espaço) como conjunto-solução de uma equação $\langle a, x\rangle=b$ (de uma inequação $\langle a, x\rangle \leqq b$ ), serão utilizados na definição geral de poliedros:

Definição 1.5 (Poliedro Geral) Dizemos que $P \subset \mathbb{R}^{n}$ é um poliedro se $P$ puder ser escrito como intersecção finita de hiperplanos e semi-espaços, isto é, se existirem matrizes $A, C$ e vetores $b, d$ tais que

$$
P=\left\{x \in \mathbb{R}^{n} \mid A x \leqq b, C x=d\right\} .
$$


Definição 1.6 (Vértice) Seja $P$ um poliedro. Dizemos que $v \in P$ é um vértice de $P$ se não existe uma direção $h \neq 0$ tal que $(x+h, x-h) \in P \times P$. Denotamos por $V(P)$ o conjunto dos vértices de $P$. de $P$ :

A um poliedro $P$ podemos associar um cone que equivale ao conjunto de direções de ilimitação

Definição 1.7 (Cone associado a $P$ ) Se $P \subset \mathbb{R}^{n}$ é um poliedro, definimos o cone associado a $P, C(P)$, por

$$
C(P)=\left\{h \in \mathbb{R}^{n} \mid(x+\alpha h) \in P, \forall x \in P, \forall \alpha \geqq 0\right\} .
$$

Note que para todo poliedro $P \neq \emptyset$, temos que $0 \in C(P)$; note ainda que 0 não é uma direção, embora falemos de $C(P)$ como o conjunto de direções de ilimitação associadas a $P$.

$\mathrm{O}$ conceito de dimensão de um poliedro é definido a seguir.

Definição 1.8 (Dimensão de um poliedro) Seja $P \subset \mathbb{R}^{n}$ um poliedro. $A$ dimensão de $P($ denotada por $\operatorname{dim}(P))$ é definida como

$$
\operatorname{dim}(P)=\min \{d \in \mathbb{N} \mid \text { existe uma variedade linear } L \text { de dimensão } d \text { tal que } P \subset L\} \text {. }
$$

\subsubsection{O Poliedro Canônico}

Um caso particular de poliedro de grande interesse é o canônico, para o qual pode-se obter de maneira simples uma caracterização algébrica para os vértices, e pode-se garantir a finitude de $V(P)$ e a existência de vértices se $P \neq \emptyset$. Além disto conhece-se uma representação alternativa (e também finita) em função de vértices e raios extremais.

Definição 1.9 (Poliedro Canônico) Um poliedro é dito canônico se puder ser representado como

$$
P=\left\{x \in \mathbb{R}^{n} \mid A x=b, x \geqq 0\right\} .
$$

Utilizando a notação $I(v)=\left\{i \mid v_{i} \neq 0\right\}$ (conjunto de índices onde $v$ é não-nulo), temos a seguinte proposição:

Proposição 1.10 (Caracterização de vértices) Seja $P$ um poliedro canônico. Então, dado $v \in P$,

$$
v \in V(P) \Longleftrightarrow\left\{A^{j}\right\}_{j \in I(v)} \text { é L. I. (linearmente independente). }
$$


Prova : A prova pode ser fragmentada em várias equivalências, cada uma de verificação imediata, usando o fato de que $v \in P$ :

$$
\begin{aligned}
v \in V(P) & \Longleftrightarrow \nexists h \neq 0:(x+h, x-h) \in P \times P \\
& \Longleftrightarrow \nexists h \neq 0: A h=0 \text { e } I(h) \subset I(v) \\
& \Longleftrightarrow \nexists h: h_{I(v)} \neq 0 \text { e } A_{I(v)} h_{I(v)}=0 \Longleftrightarrow\left\{A^{j}\right\}_{j \in I(v)} \text { é L. I. }
\end{aligned}
$$

Esta caracterização possibilita a seguinte definição:

Definição 1.11 (Solução básica) Seja $P$ um poliedro canônico, e suponha que a matriz $A$ $(m \times n)$ tem característica plena. Dado um conjunto $B$ de $m$ indices associados a colunas L.I. de $A$, dizemos que um ponto $x \in \mathbb{R}^{n}$ é uma solução básica (associada à base $B$ ), se $A x=b$ e $I(x) \subset B$. Se além disso $x \geqq 0$, dizemos que este ponto é uma solução básica viável, e consequentemente $x$ é um vértice de $P$.

Definição 1.12 (Vértice degenerado, poliedro degenerado) Seja $A$ uma matriz $m \times n$, $b \in \mathbb{R}^{m}$ e $P=\{x \mid A x=b, x \geqq 0\}$ um poliedro canônico. Dizemos que um vértice $v \in V(P)$ é degenerado se \#I $(v)<m$, caso contrário $v$ é dito não-degenerado.

Se $P=\{x \mid A x \leqq b, x \geqq 0\} \subset \mathbb{R}^{n}$ é um poliedro de dimensão $n$, dizemos que $v \in V(P)$ é degenerado se $(v, b-A x)^{t}$ é degenerado no poliedro canônico $P=\left\{(x, y)^{t} \mid A x+I y=b,(x, y)^{t} \geqq 0\right\}$.

Se $P$ é um poliedro canônico ou $P=\{x \mid A x \leqq b, x \geqq 0\} \subset \mathbb{R}^{n}$ dizemos que $P$ é nãodegenerado se todos os seus vértices são não-degenerados.

A definição de degenerescência no caso mais geral pode ser encontrada, por exemplo, em [Emelichev, Kovalev \& Kravtsov 84].

Um importante corolário de (1.10) é:

Corolário 1.13 (Finitude de $V(P))$ Se $P \neq \emptyset$ é um poliedro canônico, então $V(P)$ é finito.

Prova : Basta usar a caracterização de vértices (1.10), e notar que o número de subconjuntos de colunas de $A$ L.I. está limitado por $\sum_{i=1}^{m}\left(\begin{array}{c}n \\ i\end{array}\right)$, onde $A$ é $m \times n$.

Este último resultado (1.13) vale para poliedros em geral, ver o corolário (1.20). 
Definição 1.14 (Cone associado ao poliedro canônico) Lembrando a definição (1.7), temos que se $P$ é canônico, então

$$
C(P)=\left\{h \in \mathbb{R}^{n} \mid A h=0, h \geqq 0\right\} .
$$

Chamamos de raios extremais de $C(P)$ os vértices do poliedro

$$
C^{\prime}(P)=\left\{h \in C(P) \mid \sum_{i=1}^{n} h_{i}=1\right\} .
$$

Denotando o casco convexo de um conjunto $X$ por $[X]$, temos:

Proposição 1.15 (Representação do poliedro canônico) Seja $P$ um poliedro canônico. Então $P=[V(P)]+C(P)$.

Prova : Se $P=\emptyset$ temos que $V(P)=\emptyset$ e o resultado segue. Considere então $P \neq \emptyset$. A inclusão $[V(P)]+C(P) \subset P$ vem imediatamente das definições, pois se $x=\sum_{i=1}^{k} \alpha_{i} v^{i}+h$, onde $v^{i} \in V(P), \alpha_{i} \geqq 0, \sum_{i=1}^{k} \alpha_{i}=1$ e $h \in C(P)$, então $A\left(\sum_{i=1}^{k} \alpha_{i} v^{i}+h\right)=\sum_{i=1}^{k} \alpha_{i} A v^{i}+A h=b$ e $\sum_{i=1}^{k} \alpha_{i} v^{i}+h \geqq 0$.

Para mostrar a inclusão contrária, considere os conjuntos $P^{i}=\{x \in P \mid \# I(x) \leqq i\}$. É claro que $P^{n}=P$, e que $P^{-1}=\emptyset$.

Mostraremos por indução que $P^{i} \subset[V(P)]+C(P), \forall i \leqq n$. A base é trivial, para $i=-1$. Suponha que o resultado vale para $i \leqq p$. Se $P^{p+1}=\emptyset$, é trivial. Caso contrário, tome $x \in P^{p+1}$. Se $x$ for um vértice, então $x=x+0 \in[V(P)]+C(P)$. Considere por outro lado que $x \notin V(P)$. Neste caso, existe um $h \neq 0$ tal que $I(h) \subset I(x)$ e $(x+h, x-h) \in P \times P$. Para simplificar assuma que $h \npreceq 0$ (neste caso bastaria considerar $-h$ ạo invés de $h$ ). Temos então 2 possibilidades:

1. Se $h \geqq 0$, então $h \in C(P)$. Tomando $\alpha=\min _{h_{i}>0} x_{i} / h_{i}$ teremos que $x-\alpha h \in P$ e $\# I(x-\alpha h)<\# I(x)$, de onde $x-\alpha h \in P^{p}$ e $\alpha h \in C(P)$, logo $x=(x-\alpha h)+(\alpha h) \in P^{p}+C(P) \subset[V(P)]+C(P)+C(P)=[V(P)]+C(P)$, pois $C(P)+C(P)=C(P)$.

2. Se $h \nsupseteq 0, \quad$ tome $\quad \alpha^{-}=\min _{h_{i}>0} x_{i} / h_{i} \quad$ e $\quad \alpha^{+}=\min _{h_{i}<0} x_{i} /\left|h_{i}\right| . \quad$ Então $x^{-}=x-\alpha^{-} h \in P^{p}$ e $x^{+}=x+\alpha^{+} h \in P^{p}, \log 0$

$$
x=\frac{\alpha^{+}}{\alpha^{+}+\alpha^{-}} x^{-}+\frac{\alpha^{-}}{\alpha^{+}+\alpha^{-}} x^{+} \in\left[P^{p}\right] \subset[[V(P)]+C(P)]=[V(P)]+C(P),
$$

pois $[Z]=Z, \forall Z$ convexo, e em particular para $Z=[V(P)]+C(P)$.

Isso completa a prova. 
Da construção apresentada na última prova segue:

Corolário 1.16 (Existência de vértices) $S e \quad P \neq \emptyset$ é um poliedro canônico, então $V(P) \neq \emptyset$.

É importante mencionar que vale um resultado análogo a (1.15) para poliedros em geral:

Teorema 1.17 (Representação de poliedros) Se $P \neq \emptyset$ é um poliedro qualquer, então existe um conjunto finito $W$ e um cone $C$ tais que $P=[W]+C$. Mais ainda, se $V(P) \neq \emptyset$, então podemos escrever $P=[V(P)]+C$.

Prova : Pode ser encontrada, por exemplo, nos textos [Ziegler 95], [Ferreira \& Wakabayashi 96].

\subsubsection{Transformações de Poliedros}

Se $P \subset \mathbb{R}^{n}$ e $Q \subset \mathbb{R}^{m}$ são poliedros, então o produto cartesiano $P \times Q \subset \mathbb{R}^{n} \times \mathbb{R}^{m}$ também é um poliedro. Além disso, se $P=[V(P)]+C(P)$ e $Q=[V(Q)]+C(Q)$, temos o seguinte resultado:

\begin{tabular}{|lrrr|}
\hline Proposição 1.18 (Produto cartesiano de poliedros) $S e$ & $P=[V(P)]+C(P)$ \\
$e$ & $Q=[V(Q)]+C(Q)$ & são $\quad$ poliedros, $\quad$ então & $V(P \times Q)=V(P) \times V(Q)$, \\
$C(P \times Q)=C(P) \times C(Q)$ & e $P \times Q=[V(P \times Q)]+C(P \times Q)$ &
\end{tabular}

Prova :
1. $\left(v_{1}, v_{2}\right) \in V(P \times Q) \Longleftrightarrow \nexists\left(h_{1}, h_{2}\right) \neq(0,0)$ : $\left(\left(v_{1}+h_{1}, v_{2}+h_{2}\right),\left(v_{1}-h_{1}, v_{2}-h_{2}\right)\right) \in P \times Q$$$
\Longleftrightarrow \nexists h_{1} \neq 0:\left(v_{1}+h_{1}, v_{1}-h_{1}\right) \in P \mathrm{e}
$$$$
\nexists h_{2} \neq 0:\left(v_{2}+h_{2}, v_{2}-h_{2}\right) \in Q
$$$$
\Longleftrightarrow v_{1} \in V(P) \text { e } v_{2} \in V(Q) \text {. }
$$
2. $\left(h_{1}, h_{2}\right) \in C(P \times Q) \Longleftrightarrow \forall\left(x_{1}, x_{2}\right) \in P \times Q, \forall \alpha \geqq 0$, $\left(x_{1}+\alpha h_{1}, x_{2}+\alpha h_{2}\right) \in \bar{P} \times Q$$$
\Longleftrightarrow \forall x_{1} \in P, \forall x_{2} \in Q, \forall \alpha \geqq 0 \text {, }
$$$$
\left(x_{1}+\alpha h_{1}\right) \in P,\left(x_{2}+\alpha \bar{h}_{2}\right) \in Q
$$$$
\Longleftrightarrow h_{1} \in C(P) \text { e } h_{2} \in C(Q) \text {. }
$$

3. $P \times Q=([V(P)]+C(P)) \times([V(Q)]+C(Q))=[V(P \times Q)]+C(P \times Q)$. Imediato a partir de (i) e (ii).

Seja $\mathcal{L}$ uma transformação linear. Então $\mathcal{L}(P)$ é um poliedro, se $P$ é um poliedro. Além disso, 
Proposição 1.19 (Transformação linear de poliedros) $S e P=[V(P)]+C(P) \subset \mathbb{R}^{n}$ é um poliedro e $\mathcal{L}: \mathbb{R}^{n} \longrightarrow \mathbb{R}^{m}$ é uma transformação linear, então $V(\mathcal{L}(P)) \subset \mathcal{L}(V(P))$ e $C(\mathcal{L}(P))=\mathcal{L}(C(P))$.

Prova : Note inicialmente que

$$
\mathcal{L}(P)=\mathcal{L}([V(P)]+C(P))=\mathcal{L}([V(P)])+\mathcal{L}(C(P))=[\mathcal{L}(V(P))]+\mathcal{L}(C(P)) .
$$

1. Seja então $x \in V(\mathcal{L}(P))$. Pela última igualdade, temos que $x=\sum_{i=1}^{p} \alpha_{i} \mathcal{L}\left(v^{i}\right)+\mathcal{L}(h)$, onde $v^{i}$ são vértices de $P, \alpha>0, \quad \sum_{i=1}^{p} \alpha_{i}=1$ e $h \in C(P)$. Afirmamos que $\mathcal{L}(h)=0$ e $\mathcal{L}\left(v^{i}\right)=\mathcal{L}\left(v^{j}\right), \forall i, j: 1 \leqq i, j \leqq p$. Suponha por contradição que $\mathcal{L}(h) \neq 0$. Neste caso teríamos que $\overline{h^{\prime}}=\mathcal{L}(h)$ é uma direção tal que $\left(x+h^{\prime}, x-h^{\prime}\right) \in \mathcal{L}(P) \times \mathcal{L}(P)$, e conseqüentemente $x$ não seria vértice. Isso mostra que $\mathcal{L}(h)=0$. Suponha agora por contradição que tenhamos $\mathcal{L}\left(v^{i}\right) \neq \mathcal{L}\left(v^{j}\right)$ para algum $i \neq j$. Então, como $\alpha>0, \quad h^{\prime}=\min \left\{\alpha_{i}, \alpha_{j}\right\}\left(v^{i}-v^{j}\right) \neq 0$ é uma direção tal que $\left(x+h^{\prime}, x-h^{\prime}\right) \in \mathcal{L}(P) \times \mathcal{L}(P)$, contradizendo o fato de $x$ ser vértice. Isto mostra que $x=\sum_{i=1}^{p} \alpha_{i} \mathcal{L}\left(v^{i}\right)=\mathcal{L}\left(v^{1}\right)$, ou seja, $x \in \mathcal{L}(V(P))$. Isto mostra que $V(\mathcal{L}(P)) \subset \mathcal{L}(V(P))$

2. Para provar a afirmação sobre os cones, mostraremos antes que $V(P)$ é um conjunto finito. Note que como $P$ é um poliedro, então ele pode ser representado na forma

$$
P=\left\{x \in \mathbb{R}^{n} \mid A x \leqq b, C x=d\right\}
$$

e pode ser obtido por uma transformação linear $\mathcal{M}$ do seguinte poliedro canônico:

$$
P^{\prime}=\left\{\begin{array}{l|l}
\left(x^{+}, x^{-}, x^{r}\right)^{t} \in \mathbb{R}^{n} \times \mathbb{R}^{n} \times \mathbb{R}^{m} & \begin{array}{l}
A x^{+}-A x^{-}+I x^{r}=b, \\
C x^{+}-C x^{-}=d, \\
\left(x^{+}, x^{-}, x^{r}\right)^{t} \geqq 0
\end{array}
\end{array}\right\} ;
$$

basta fazer $\mathcal{M}\left(x^{+}, x^{-}, x^{r}\right)=x^{+}-x^{-}$. Pelo corolário (1.13) sabemos que $V\left(P^{\prime}\right)$ é finito, e pelo item 1 sabemos que $V(P)=V\left(\mathcal{M}\left(P^{\prime}\right)\right) \subset \mathcal{M}\left(V\left(P^{\prime}\right)\right), \operatorname{logo} V(P)$ é finito.

3. Seja $h \in \mathcal{L}(C(P))$. Note que $h \in \mathcal{L}(C(P)) \Longleftrightarrow h=\mathcal{L}\left(h^{\prime}\right)$, onde $\quad h^{\prime} \in C(P)$. Então $x+\alpha h^{\prime} \in P, \forall x \in P, \forall \alpha \geqq 0, \quad \operatorname{logo}$ $\mathcal{L}(x)+\alpha \mathcal{L}\left(h^{\prime}\right) \in \mathcal{L}(P), \forall x \in P, \forall \alpha \geqq 0$. Isso mostra que $h=\mathcal{L}\left(h^{\prime}\right) \in C(\mathcal{L}(P))$. Por outro lado, como $\mathcal{L}(P)=[\mathcal{L}(\overline{\bar{V}}(P))]+\mathcal{L}(C(P))$ e $V(P)$ é finito, então $[\mathcal{L}(V(P))]$ é limitado, logo $C(\mathcal{L}(P)) \subset \mathcal{L}(C(P))$. Isto conclui a prova.

Decorre da demonstração anterior (item 2) que:

Corolário 1.20 (Finitude de $V(P)$ : caso geral) Se $P \subset \mathbb{R}^{n}$ é um poliedro qualquer então $V(P)$ é finito. 


\subsection{Programação Linear}

Nesta seção estaremos considerando o problema de minimizar uma função linear sobre um poliedro. Para problemas deste tipo vale o seguinte resultado:

Proposição 1.21 (Existência de vértice ótimo) Seja $f$ uma função côncava. Se o problema

$$
\left\{\begin{array}{cl}
\min & f(x) \\
\text { s.a. } & x \in[V(P)]+C(P)
\end{array}\right.
$$

admite solução ótima, então existe um vértice que é ótimo.

Prova: Inicialmente note que $f(x+h) \geqq f(x), \forall x \in P, \forall h \in C(P)$, pois do contrário teríamos ao longo de $h$ uma direção de ilimitação da função objetivo, contradizendo a existência de solução ótima. Por outro lado, para todo $x \in P$, podemos escrever $x=\sum_{i=1}^{p} \alpha_{i} v^{i}+h$, e como $f$ é côncava,

$$
\begin{aligned}
f(x) & =f\left(\sum_{i=1}^{p} \alpha_{i} v^{i}+h\right) \\
& \geqq f\left(\sum_{i=1}^{p} \alpha_{i} v^{i}\right) \\
& \geqq \sum_{i=1}^{p} \alpha_{i} f\left(v^{i}\right) \\
& \geqq \min _{1} \leqq i \leqq p
\end{aligned}
$$

Logo $f\left(v^{\min }\right) \leqq f\left(x^{*}\right)$, se $x^{*}$ é uma solução ótima.

Como toda função linear é côncava, segue o mesmo resultado para o problema canônico de programação linear, definido a seguir:

Definição 1.22 (O problema canônico de programação linear) Seja $A$ uma matriz $m \times n$ com característica plena. Definimos:

$$
(P L C)\left\{\begin{array}{cl}
\min & \langle c, x\rangle \\
\text { s.a. } & A x=b \\
& x \geqq 0 .
\end{array}\right.
$$

Lembrando a proposição (1.10), sabemos que a todo vértice $v$ podemos associar o conjunto de colunas $I(v)$, e se a matriz $A$ tem característica plena então podemos associar um conjunto $B$ dos índices de $m$ colunas L.I. extraidas de $A$, com $I(v) \subset B$. Um conjunto assim é dito uma base representando o vértice $v$, e denotamos por $v_{B}$ a restrição do vetor $v$ às coordenadas da base $B$, e $\tilde{v}$ a restrição às coordenadas não-básicas, isto é, que não estão em $B$.

Proposição 1.23 (Teste de otimalidade) Seja $v \in V(P), \quad$ e $B \quad \subset \quad\{1,2, \ldots, n\}$ um conjunto de $m$ colunas da matriz $A$, linearmente independentes, tais que $I(v) \subset B$. Se $\tilde{\gamma}_{j}=c_{j}-\left\langle A_{B}^{-1} A^{j}, c_{B}\right\rangle \geqq 0, \forall j \notin B$, então $v$ é otimo. 
Prova : Seja $x \in P$ qualquer. Denote por $\tilde{x}=\left(x_{i}\right)_{i \notin B}$. Então

$$
\begin{aligned}
\langle c, x\rangle & =\left\langle c_{B}, x_{B}\right\rangle+\langle\tilde{c}, \tilde{x}\rangle \\
& =\left\langle c_{B}, A_{B}^{-1}(b-\tilde{A} \tilde{x})\right\rangle+\langle\tilde{c}, \tilde{x}\rangle \\
& =\left\langle c_{B}, A_{B}^{-1} b\right\rangle+\left\langle\tilde{c}-\left(A_{B}^{-1} \tilde{A}\right)^{t} c_{B}, \tilde{x}\right\rangle \\
& =\left\langle c_{B}, A_{B}^{-1} b\right\rangle+\{\tilde{\gamma}, \tilde{x}\rangle \\
& \geqq\left\langle c_{B}, A_{B}^{-1} b\right\rangle \\
& =\langle c, v\rangle .
\end{aligned}
$$

onde $\{\tilde{\gamma}, \tilde{x}\rangle \geqq 0$, pois $\tilde{\gamma} \geqq 0$ e $\tilde{x} \geqq 0$. Logo $v$ é ótimo.

Note que definindo $\gamma=c-\left(A_{B}^{-1} A\right)^{t} c_{B}$, temos automaticamente $\gamma_{j}=0$ para $j \in B$.

Sob determinados cuidados (por exemplo, regra de Bland), podemos passar de um vértice não-ótimo para um outro vértice, no mínimo tão bom quanto o anterior, e de maneira a nunca repetir uma base. Esta é a ideia central do método simplex, cuja descrição detalhada pode ser encontrada em vários textos, como [Bazaraa \& Jarvis 77], [Dantzig 63], [Humes \& Humes 86] e [Luenberger 73].

\subsubsection{Dualidade em Programação Linear}

É fácil ver que o problema (PLC) definido em (1.22) pode ser expresso como

$$
\min _{x \geqq 0} \sup _{u \in \mathbb{R}^{m}}\langle c, x\rangle+\langle b-A x, u\rangle .
$$

ou ainda

$$
\min _{x \geqq 0} \sup _{u \in \mathbb{R}^{m}}\langle b, u\rangle+\left\langle c-A^{t} u, x\right\rangle
$$

Estamos interessados em relacionar então o (PLC) com um outro problema, chamado de dual do (PLC), definido através da seguinte expressão:

$$
\max _{u \in \mathbb{R}^{m}} \inf _{x \geqq 0}\langle b, u\rangle+\left\langle c-A^{t} u, x\right\rangle .
$$

que corresponde a

Definição 1.24 (O problema linear dual)

$$
(D L C)\left\{\begin{aligned}
\max & \langle b, u\rangle \\
s . a . & A^{t} u \leqq c
\end{aligned}\right.
$$

A relação entre os problemas (PLC) e (DLC) admite belas interpretações econômicas, que podem ser encontradas em [Bazaraa \& Jarvis 77], [Humes \& Humes 86], [Dantzig 63]. É importante frisar que o problema dual pode ser definido em geral para problemas de programação matemática, não necessariamente lineares.

Uma importante relação entre os problemas primal e dual em programação linear é que o dual do problema (DLC) é equivalente ao próprio (PLC), fato este que será usado adiante no teorema forte de dualidade (1.26).

Os dois principais resultados que iremos utilizar são: 
Teorema 1.25 (Teorema fraco de dualidade) Se $x$ é viável no (PLC) e u é viável no $(D L C)$, então $\langle c, x\rangle \geqq\langle b, u\rangle$.

Prova : $\langle c, x\rangle \geqq\left\langle A^{t} u, x\right\rangle=\langle u, A x\rangle=\langle u, b\rangle$, pois $\left(c-A^{t} u\right) \geqq 0$ e $x \geqq 0$.

Teorema 1.26 (Teorema forte de dualidade)

$$
\inf \{\langle c, x\rangle \mid A x=b, x \geqq 0\} \geqq \sup \left\{\langle b, u\rangle \mid A^{t} u \leqq c\right\}
$$

Mais ainda, se um dos problemas é viável, então vale a igualdade.

Prova : A desigualdade é consequência de (1.25), com a convenção inf $\emptyset=+\infty$ e $\sup \emptyset=-\infty$. Suponha que um dos problemas é viável. Temos três possibilidades:

1. Se um dos problemas é ilimitado, então o outro é inviável, pelo teorema fraco (1.25), e vale a igualdade;

2. Se o primal tem solução ótima, então ele possui um vértice $v$ ótimo associado à base B, por (1.21), e $\gamma=c-\left(A_{B}^{-1} A\right)^{t} c_{B} \geqq 0$. Definindo $u=\left(A_{B}^{-1}\right)^{t} c_{B}$, temos que $A^{t} u \leqq c$ e $\langle u, b\rangle=\left\langle\left(A_{B}^{-1}\right)^{t} c_{B}, b\right\rangle=\left\langle c_{B}, A_{B}^{-1} b\right\rangle=\langle c, v\rangle$, o que mostra que $u$ é ótimo para o (DLC), portanto vale a igualdade; $\mathrm{O}$ vetor $u$ aqui definido é também chamado de multiplicador dual associado à solução $v$.

3. Se o dual tem solução ótima, então existe um vértice ótimo para o problema

$$
\left\{\begin{aligned}
\max & \left\langle b, u^{+}\right\rangle-\left\langle b, u^{-}\right\rangle \\
\text {s.a. } & A^{t} u^{+}-A^{t} u^{-}+u^{r}=c \\
& u^{+}, u^{-}, u^{r} \geqq 0 .
\end{aligned}\right.
$$

cujo dual por (1.24) é equivalente ao próprio (PLC). Então usando o item (2) temos o resultado.

A partir do teorema forte de dualidade, podemos explicar o algoritmo simplex como um algoritmo que mantém a cada passo a viabilidade primal de $x$, e uma condição chamada de folgas complementares, que para este problema equivale a $x_{j}\left(c_{j}-A_{j}^{t} u\right)=0$, para $j=1, \ldots, n$, onde $u=\left(A_{B}^{-1}\right)^{t} c_{B}$; com esta invariante, o algorítmo simplex procura atingir viabilidade dual para $u$. Outra estratégia possível, manter viabilidade dual e folgas complementares, procurando atingir viabilidade primal, dá origem ao método conhecido como simplex dual, que pode ser encontrado nos textos [Bazaraa \& Jarvis 77], [Dantzig 63], [Humes \& Humes 86] e [Luenberger 73].

Uma aplicação interessante dos teoremas de dualidade que nos será útil é o seguinte teorema:

Teorema 1.27 (Teorema de alternativa de Gordan) Considere os sistemas

$$
\begin{array}{ll}
\text { (1) }\{A x=0, x \geq 0 & \text { (2) }\left\{A^{t} u>0\right.
\end{array}
$$

Então (1) admite solução $\Longleftrightarrow$ (2) não admite solução. 
Prova : Seja $\overline{1}=(1,1, \ldots, 1)^{t}$. Considere os problemas primal e dual dados por

$$
(P L C)\left\{\begin{array} { r l } 
{ \operatorname { m a x } } & { \langle 1 , x \rangle } \\
{ \text { s.a. } } & { A x = 0 } \\
{ } & { x \geqq 0 }
\end{array} \quad ( D L C ) \left\{\begin{array}{rl}
\min & \langle 0, u\rangle \\
\text { s.a. } & A^{t} u \geqq \overline{1}
\end{array}\right.\right.
$$

Então, usando o teorema (1.26),

1. (1) admite solução $\Longrightarrow($ PLC) é ilimitado $\Longrightarrow(D L C)$ é inviável $\Longrightarrow(2)$ não admite solução.

2. (1) não admite solução $\Longrightarrow(P L C)$ tem solução ótima $\Longrightarrow$ (DLC) tem solução ótima $\Longrightarrow(2)$ admite solução.

\subsubsection{Decomposição de Dantzig-Wolfe}

Neste seção mostraremos um método para a resolução de problemas lineares onde a matriz $A$ tem uma estrutura bloco-diagonal. Consideraremos o caso em que a matriz tem dois blocos; o caso geral pode ser encontrado, por exemplo, em [Dantzig \& Wolfe 60], [Dantzig 63], [Humes 78], [Bazaraa \& Jarvis 77].

Definição 1.28 (Problema bloco-diagonal: 2 blocos) Para $i=1,2$ sejam $A^{i}$ matrizes $m_{i} \times n_{i}, L^{i}$ matrizes $m_{3} \times n_{i}, c^{i} \in \mathbb{R}^{n_{i}}$, e $b^{i} \in \mathbb{R}^{m_{i}}$, para $i=1,2,3$. Definimos:

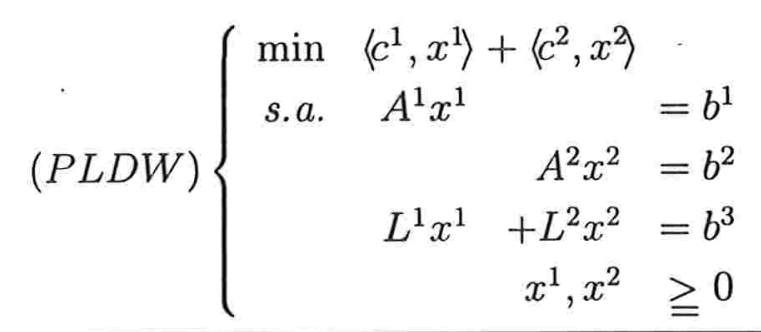

Sejam $V^{1}=\left\{v^{1}, v^{2}, \ldots, v^{k_{1}}\right\} \quad$ e $\quad V^{2}=\left\{w^{1}, w^{2}, \ldots, w^{k_{2}}\right\} \quad$ o conjunto de vértices de $P^{1}=\left\{x \mid A^{1} x^{1}=b^{1}, x^{1} \geqq 0\right\} \quad$ e $\quad P^{2}=\left\{x \mid A^{2} x^{2}=b^{2}, x^{2} \geqq 0\right\}$, respectivamente. Sejam $C^{1}=\left\{v^{k_{1}+1}, v^{k_{1}+2}, \ldots, v^{\overline{l_{1}}}\right\}$ e $C^{2}=\left\{w^{k_{2}+1}, w^{k_{2}+2}, \ldots, w^{l_{2}}\right\}$ os raios extremais associados a estes poliedros.

Então o problema original (1.28) é equivalente a

$$
\left\{\begin{aligned}
\min \quad \sum_{i=1}^{l_{1}}\left(\left\langle c^{1}, v^{i}\right\rangle\right) \alpha_{i}+\sum_{j=1}^{l_{2}}\left(\left\langle c^{2}, w^{j}\right\rangle\right) \beta_{j} & \\
\text { s.a. } \quad \sum_{i=1}^{k_{1}} \alpha_{i} & =1 \\
\sum_{j=1}^{k_{2}} \beta_{j} & =1 \\
\sum_{i=1}^{l_{1}}\left(L_{1} v^{i}\right) \alpha_{i}+\sum_{j=1}^{l_{2}}\left(L_{1} w^{j}\right) \beta_{j} & =b^{3} \\
\alpha, \beta & \geqq 0
\end{aligned}\right.
$$


Vamos assumir que a matriz deste sistema linear tem característica plena, e portanto poderemos falar em soluções básicas, como foi visto na seção (1.1).

Seja $\left(\overline{x^{1}}, \overline{x^{2}}\right)=\left(\sum_{i=1}^{l_{1}} \overline{\alpha_{i}} v^{i}, \sum_{j=1}^{l_{2}} \overline{\beta_{j}} w^{j}\right)$ uma solução básica associada à base $\mathcal{B}=\left(B_{1}, B_{2}\right)$ para o problema (1), isto é, tais que as $\left(m_{3}+2\right)$ colunas

$$
\left\{\left[\begin{array}{c}
1 \\
0 \\
L^{1} v^{i_{1}}
\end{array}\right],\left[\begin{array}{c}
0 \\
0 \\
L^{1} v^{i_{2}}
\end{array}\right],\left[\begin{array}{c}
0 \\
1 \\
L^{2} w^{j_{1}}
\end{array}\right],\left[\begin{array}{c}
0 \\
0 \\
L^{2} w^{j_{1}}
\end{array}\right] \begin{array}{c}
i_{1} \in B_{1}, i_{1} \leqq k_{1} \\
i_{2} \in B_{1}, i_{2}>k_{1} \\
j_{1} \in B_{2}, j_{1} \leqq k_{2} \\
j_{2} \in B_{2}, j_{2}>k_{2}
\end{array}\right\}
$$

sejam L.I. Seja $(s, t, u)$ o multiplicador dual (ver (1.26)) associado a esta solução, e defina $\gamma^{i}=c^{i}-\left(L^{i}\right)^{t} u, i=1,2$. Então podemos afirmar:

Teorema 1.29 (Teste de otimalidade para Dantzig-Wolfe) A solução básica $\left(\overline{x^{1}}, \overline{x^{2}}\right)$ é ótima se

$$
\begin{aligned}
& s=\min \left\langle\gamma^{1}, x^{1}\right\rangle \quad, \text { et } t=\max \left\langle\gamma^{2}, x^{2}\right\rangle \text {, } \\
& \text { s.a. } A^{1} x^{1}=b^{1} \quad \text { s.a. } \quad A^{2} x^{2}=b^{2} \\
& x^{1} \geqq 0 \quad x^{2} \geqq 0
\end{aligned}
$$

onde $\gamma^{1}=c^{1}-\left(L^{1}\right)^{t} u$ e $\gamma^{2}=c^{2}-\left(L^{2}\right)^{t} u$.

Prova : Como $(s, t, u)$ é o multiplicador associado à solução $\left(\overline{x^{1}}, \overline{x^{2}}\right)$, ele tem que satisfazer às igualdades:

$$
\begin{aligned}
s+\left(L^{1} v^{i}\right)^{t} u & =\left\langle c^{1}, v^{\imath}\right\rangle, \quad \forall i \in B_{1}, i \leqq k_{1} \\
\left(L^{1} v^{i}\right)^{t} u & =\left\langle c^{1}, v^{\imath}\right\rangle, \quad \forall i \in B_{1}, i>k_{1} \\
t+\left(L^{1} w^{j}\right)^{t} u & =\left\langle c^{2}, w^{\jmath}\right\rangle, \quad \forall j \in B_{2}, j \leqq k_{2} \\
\left(L^{1} w^{j}\right)^{t} u & =\left\langle c^{2}, w^{\jmath}\right\rangle, \quad \forall j \in B_{2}, j>k_{2}
\end{aligned}
$$

Além disso, $\left\langle\gamma^{1}, \overline{x^{1}}\right\rangle=s,\left\langle\gamma^{2}, \overline{x^{2}}\right\rangle=t$, pois

$$
\left\langle\gamma^{i}, \overline{x^{i}}\right\rangle=\left\langle c^{i}-\left(L^{i}\right)^{t} u, \sum_{j=1}^{l_{i}} \overline{\alpha_{j}} v^{j}\right\rangle=\sum_{j=1}^{l_{i}} \overline{\alpha_{j}}\left(\left\langle c^{i}, v^{j}\right\rangle-\left\langle u, L^{i} v^{j}\right\rangle\right)= \begin{cases}s, & \text { se } i=1 \\ t, & \text { se } i=2\end{cases}
$$

por (2); Multiplicando a terceira equação de (PLDW) (1.28) por $u^{t}$ obtemos, para todo par $\left(x^{1}, x^{2}\right)$ viável, $u^{t} b^{3}=u^{t} L^{1} x^{1}+u^{t} L^{2} x^{2}=\left(c^{1}-\gamma^{1}\right) x^{1}+\left(c^{2}-\gamma^{2}\right) x^{2}$, de onde concluimos que, para todo par $\left(x^{1}, x^{2}\right)$ viável,

$$
\left(\left\langle c^{1}, \overline{x^{1}}\right\rangle+\left\langle c^{2}, \overline{x^{2}}\right\rangle\right)-\left(\left\langle c^{1}, x^{1}\right\rangle+\left\langle c^{2}, x^{2}\right\rangle\right)=s+t-\left\langle\gamma^{1}, x^{1}\right\rangle-\left\langle\gamma^{2}, x^{2}\right\rangle \leqq 0 .
$$

A última desigualdade segue da hipótese da proposição. Disto concluimos que $\left\langle c^{1}, \overline{x^{1}}\right\rangle+\left\langle c^{2}, \overline{x^{2}}\right\rangle \leqq\left\langle c^{1}, x^{1}\right\rangle+\left\langle c^{2}, x^{2}\right\rangle$ para qualquer $\left(x^{1}, x^{2}\right)$ viável, logo $\left(\overline{x^{1}}, \overline{x^{2}}\right)$ é ótimo. 
Se o ponto $\left(\overline{x^{1}}, \overline{x^{2}}\right)$ não satisfaz o teste de otimalidade (1.29) então existe, sem perda de generalidade, um vértice ótimo $v^{*}$ em $P^{1}$ tal que $\left\langle\gamma^{1}, v^{*}\right\rangle<s$ ou uma direção de ilimitação $h^{*}$ tal que $\left\langle\gamma^{1}, h^{*}\right\rangle<0, A^{1} h^{*}=0$. Em ambos os casos a variável associada a esta nova coluna será uma candidata a entrar na base, substituindo uma das $\left(m_{3}+2\right)$ colunas na base $\mathcal{B}$, pois

$$
\begin{aligned}
\gamma^{\text {novo }} & =\left\langle c^{1}, v^{*}\right\rangle-\left\langle\left[\begin{array}{c}
0 / 1 \\
0 \\
L^{1} v^{*}
\end{array}\right],\left[\begin{array}{c}
s \\
t \\
u
\end{array}\right]\right\rangle \\
& =\left\{\begin{array}{llll}
\left\langle c^{1}, v^{*}\right\rangle-s-\left\langle L^{1} v^{*}, u\right\rangle=\left\langle c^{1}, v^{*}\right\rangle-s-\left\langle v^{*}, c^{1}-\gamma^{1}\right\rangle & <0 & \text { se } v^{*} \text { é vértice; } \\
\left\langle c^{1}, h^{*}\right\rangle-\left\langle L^{1} h^{*}, u\right\rangle & =\left\langle c^{1}, h^{*}\right\rangle-\left\langle h^{*}, c^{1}-\gamma^{1}\right\rangle & <0 & \text { se } h^{*} \text { é dir. de ilimitação. }
\end{array}\right.
\end{aligned}
$$

Com este método podemos conhecer a cada passo apenas $m_{3}+2$ colunas que estão na base, ao passo que o simplex aplicado diretamente ao problema original (1.28) precisaria conhecer a cada passo $m_{1}+m_{2}+m_{3}$ colunas.

\subsection{Geometria Poliédrica}

Nesta seção definiremos alguns conceitos geométricos associados a poliedros, tais como faces e arestas, e mostraremos que sob certas condições podemos representar um poliedro apenas com desigualdades associadas a facetas.

Definição 1.30 (Faces, vértices, vértices vizinhos, arestas, facetas) Seja $P$ um poliedro não-vazio, e $\langle a, x\rangle \geqq b$ um semi-espaço que contém $P$. Então o conjunto $F=P \cap\{\langle a, x\rangle=b\}$ é denominado face de $P$ gerada por $\langle a, x\rangle \geqq b$.

Verificaremos a seguir que as faces de dimensão 0 são vértices.

Às faces de dimensão 1 damos o nome de arestas.

Dois vértices $u, v \in V(P)$ são ditos vizinhos se $[u, v]$ é uma aresta.

Se $P \subset \mathbb{R}^{n}$ tem dimensão $q$, então denominamos facetas as faces de dimensão $q-1$.

Proposição 1.31 (Vértices como faces de dimensão 0)

$v \in V(P) \Longleftrightarrow \exists a, b$ tais que $P \subset\{\langle a, x\rangle \geqq b\}$ e $\{v\}=P \cap\{\langle a, x\rangle=b\}$.

\section{Prova :}

$(\Longrightarrow)$ Seja $v^{\bar{\imath}} \in V(P)=\left\{v^{1}, v^{2}, \ldots, v^{k}\right\}$, e sejam $\left\{h^{1}, h^{2}, \ldots, h^{l}\right\}$ os raios extremais de $C(P)$. Então o sistema

$$
\sum_{i=1, i \neq \bar{\imath}}^{k} \alpha_{i}\left(v^{i}-v^{\bar{\imath}}\right)+\sum_{j=1}^{l} \beta_{j} h^{j}=0,\left[\begin{array}{l}
\alpha \\
\beta
\end{array}\right] \geq 0
$$

não admite solução. Logo por Gordan (1.27) o sistema

$$
\left\langle v^{i}-v^{\overline{2}}, \phi_{\rangle}>0,\left\langle h^{j}, \phi\right\rangle>0, \forall i \neq \bar{\imath}, \forall j\right.
$$


admite uma solução $\bar{c}$. Então $\left\langle\bar{c}, v^{\imath}\right\rangle>\left\langle\bar{c}, v^{\bar{\eta}}\right\rangle, \forall i \neq \bar{\imath}$ e $\left\langle\bar{c}, h^{j}\right\rangle>0, \forall j$, o que mostra que $\langle\bar{c}, x\rangle>\left\langle\bar{c}, v^{\bar{\gamma}}, \forall x \in P, x \neq v^{\bar{\imath}}\right.$, logo $P \cap\left\{\langle\bar{c}, x\rangle=\left\langle\bar{c}, v^{\bar{\tau}}\right\rangle\right\}=\left\{v^{\bar{\imath}}\right\}$.

$(\Longleftarrow)$ Suponha, por contradição, que exista $h \neq 0$ tal que $(v+h, v-h) \in P \times P$. Então, como $P \subset\{\langle a, x\rangle \geqq b\}$, temos que $\langle a, v+h\rangle>\langle a, v\rangle$ e $\langle a, v-h\rangle>\langle a, v\rangle$, de onde concluimos que $0<\langle a, h\rangle<0$. Esta contradição mostra que $v \in V(P)$.

Introduziremos agora algumas denominações que serão utilizadas na discussão que segue:

Definição 1.32 (Direção viável e aresta saindo de um vértice) Seja $P$ um poliedro $e$ $v \in V(P)$. Dizemos que $h \neq 0$ é uma direção viável saindo de $v$ se existe $\varepsilon>0$ tal que $[v, v+\varepsilon h] \subset P$.

Dizemos que $d \neq 0$ é uma direção de aresta saindo de $v$ se $P \cap\{v+\alpha d \mid \alpha \geqq 0\}$ é uma aresta de $P$.

É fácil notar que o conjunto das direções viáveis saindo de $v$ (unido a $\{0\}$ ) é um cone convexo, ou seja, é um conjunto fechado em relação à soma e à multiplicação por um escalar não-negativo. Esta propriedade será utilizada na seguinte caracterização geométrica de arestas: uma direção viável saindo de um vértice é uma (direção de) aresta se não puder ser escrita como combinação linear positiva de outras direções viáveis saindo do mesmo vértice.

Proposição 1.33 (Caracterização de arestas) Seja $v \in V(P)$, ed $\neq 0$ uma direção viável a partir de $v$. Então

$d$ é direção de aresta saindo de $v$

$\Uparrow$

$\nexists\left\{h^{j}\right\}_{j=1}^{k}, \nexists \beta \geq 0:\{d\} \cup\left\{h^{j}\right\}_{j=1}^{k}$ é L.I. e $h^{j}$ é dir. viável a partir de $v, \forall j e$ $d=\sum_{j=1}^{k} \beta_{j} h^{j}$.

\section{Prova :}

$(\Longrightarrow)$ Seja $\langle a, x\rangle \geqq b$ o semi-espaço que gera a aresta $d$ saindo de $v$, e suponha por contradição que existam $\left\{h^{j}\right\}_{j=1}^{k}$, e $\beta \geq 0$ como no enunciado da proposição. Como $h^{j} \neq d, \forall d$, existem pelo menos dois coeficientes $\beta_{i}$ e $\beta_{j}$ não-nulos, com $h^{i} \neq h^{j}$. Mas as direções $h^{i}$ e $h^{j}$ são viáveis; usando o fato do conjunto de direções viáveis a partir de $v$ ser um cone convexo, se $\bar{\beta}=\min \left\{\beta_{i}, \beta_{j}\right\}$, então as direções $d^{1}=d-\bar{\beta} h^{i}+\bar{\beta} h^{j}$ e $d^{2}=d+\bar{\beta} h^{i}-\bar{\beta} h^{j}$ são viáveis, e $d^{1} \neq d$ e $d^{2} \neq d$. Logo pelo fato de $d$ ser uma direção de aresta saindo de $v$, teremos $\left\langle a, d^{1}\right\rangle>0$ e $\left\langle a, d^{2}\right\rangle>0$, de onde concluimos que $\langle a, d\rangle=\left\langle a, 0.5 d^{1}+0.5 d^{2}\right\rangle>0$; isto contradiz o fato de $d$ ser uma direção de aresta saindo de $v$, gerada por $\{\langle a, x\rangle=b\}$. A contradição mostra que não podem existir $\left\{h^{j}\right\}_{j=1}^{k}$, e $\beta \geq 0$ como no enunciado da proposição. 
$(\Longleftarrow)$ Seja $V(P)=\left\{v^{1}, \ldots, v^{p}\right\}$ e $v=v^{\overline{ }}$. Seja também $\left\{h^{j}\right\}_{j=1}^{l}$ o conjunto de raios extremais do cone $C(P)$. Analogamente ao que foi feito em (1.31), podemos construir o conjunto

$$
X=\left\{v^{i}-v \mid\left\{v^{i}-v, d\right\} \text { é L.I. }\right\} \cup\left\{h^{j} \mid\left\{h^{j}, d\right\} \text { é L.I. }\right\},
$$

e notamos que não podem existir $\beta \geq 0, \eta \geq 0$, e $\zeta \in \mathbb{R}$ tais que

$$
\sum_{i=1, i \neq \bar{\imath}}^{p} \beta_{i}\left(v^{i}-v^{\bar{\imath}}\right)+\sum_{j=1}^{l} \eta_{j} h^{j}+\zeta d=0
$$

Suponha por contradição que existissem $\beta \geq 0, \eta \geq 0, \zeta \in \mathbb{R}$ satisfazendo a equação acima. Teríamos 3 casos:

1. Se $\zeta=0$ teríamos que $v^{\bar{\imath}}$ não seria vértice, como em (1.31).

2. Se $\zeta>0$ então concluiríamos que $-d$ é direção viável, pois $(-d)=\sum_{i=1, i \neq \bar{\imath}}^{p} \frac{\beta_{i}}{\zeta}\left(v^{i}-v^{\bar{\imath}}\right)+\sum_{j=1}^{l} \frac{\eta_{j}}{\zeta} h^{j}$; isso contradiz o fato de $v$ ser vértice.

3. Se $\zeta<0$, então teríamos $d=\sum_{i=1, i \neq i}^{p} \frac{\beta_{i}}{\zeta}\left(v^{i}-v^{\bar{\imath}}\right)+\sum_{j=1}^{l} \frac{\eta_{j}}{\zeta} h^{j} ;$ isso contradiz a hipótese.

Já que o sistema anterior não possui solução, então o sistema alternativo:

$$
\begin{gathered}
\left\langle v^{i}-v^{\bar{i}}, \phi\right\rangle 0, \forall i \neq \bar{\imath} \\
\left\langle h^{j}, \phi\right\rangle>0, \forall j, \\
\langle d, \phi=0
\end{gathered}
$$

admite uma solução $\bar{c}$. Logo, $\left.\quad\left\langle\bar{c}, v^{2}\right\rangle\right\rangle\left\langle\bar{c}, v^{\bar{\gamma}}\right\rangle, \forall i \neq \bar{\imath}$ e $\left\langle\bar{c}, h^{j}\right\rangle>0, \forall j$, o que mostra que $\langle\bar{c}, x\rangle>\left\langle\bar{c}, v^{\bar{\gamma}}\right\rangle, \forall x \in P \backslash\{v+\alpha d \mid \alpha \geqq 0\}$, e $\langle\bar{c}, x\rangle=\langle\bar{c}, v\rangle$, $\forall x \in\{v+\alpha d \mid \alpha \geqq 0\}$, logo $P \cap\{\langle\bar{c}, x\rangle=\langle\bar{c}, v\rangle\} \subset\{v+\alpha \bar{d} \mid \alpha \geqq 0\}$; disto concluimos que $d$ é uma direção de aresta saindo de $v$, gerada por $\{\langle\bar{c}, x\rangle \geqq\langle\bar{c}, v\rangle\}$.

Esta proposição motiva o seguinte método para o cômputo de todas as arestas saindo de um dado vértice $v$ :

Algorítmo 1 (Método inocente para o cômputo de arestas) Seja $P=[V(P)]+C(P)$ um poliedro e $v \in V(P)$. Considere o método descrito abaixo:

1. Compute $X=\left\{v^{i}-v \mid v^{i} \in V(P), v^{i} \neq v\right\} \cup\{h \mid h$ é raio extremal de $C(P)\}$.

2. Para cada $d \in X$, verifique se existe $\alpha \geq 0$ tal que $d=\sum_{d^{i} \in X, d^{i} \neq d} \alpha_{i} d^{i}$. Em caso afirmativo, faça $X \longleftarrow X \backslash\{d\}$.

3. Devolva $X$.

Este método devolve em $X$ o conjunto das (direções de) arestas saindo de $v$. 
Prova : É fácil verificar que o conjunto $X$ calculado no passo 1 fornece uma aproximação cônica de $P$ a partir de $v$; isto é,

$$
P \subset\left\{x \in \mathbb{R}^{n} \mid x=v+\sum_{i=1}^{k} \beta_{i} h^{i}, \beta \geqq 0, h^{i} \in X, i=1, \ldots, k\right\} .
$$

Isto é verdade pois se $x \in P$, então $x=\sum_{i=1}^{k_{1}} \alpha_{i} v^{i}+h$, onde $h \in C(P)$; daí

$$
x=v+\sum_{i=1}^{k_{1}}\left(v^{i}-v\right)+\sum_{j=1}^{k_{2}} \beta_{j} h^{j}
$$

onde $h^{j}$ são raios extremais de $P$, e $\left(v^{i}-v\right) \in X$.

A propriedade acima não é alterada pelo passo 2, uma vez que são eliminadas apenas direções que apontam para $\operatorname{Int}(P)$, portanto apenas direções que podem ser obtidas por sua vez como combinações lineares positivas das demais direções em $X$.

Isso garante que no passo 3 o conjunto $X$ contém somente direções positivamente linearmente independentes, que por (1.33) são direções de arestas. Mas $X$ fornece uma aproximação cônica de $P$ a partir de $v$, então $X$ possui todas as direções de arestas saindo de $v$.

Outra conseqüência da proposição (1.33) é a seguinte:

Proposição 1.34 (Número de arestas saindo de $v$ : poliedro canônico) Seja $P$ o poliedro não-degenerado dado por $P=\left\{x \in \mathbb{R}^{n} \mid A x=b, x \geqq 0\right\}$, onde $A$ é uma matriz $m \times n$ de característica plena. Então a partir de qualquer vértice $v \in V(P)$ saem exatamente $n-m$ arestas.

Prova : Como $P$ é não-degenerado sabemos que $\# I(v)=m$, e que $\left\{A^{j}\right\}_{j \in I(v)}$ é L.I. Então $v$ é uma solução básica viável associada a $B=I(v)$; assuma que $B=\{1, \ldots, m\}$ para simplificar a notação. Para cada $j \notin I(v)$, a direção

$$
d^{j}=\left[\begin{array}{c}
-A_{B}^{-1} A^{j} \\
0 \\
\vdots \\
1 \\
0 \\
\vdots \\
0
\end{array}\right]
$$

onde 1 aparece na j-ésima coordenada, é viável saindo de $v$ (possivelmente ilimitada); o conjunto de direções $\left\{d^{j}\right\}_{j \notin I(v)}$ é L.I. trivialmente e além disto qualquer direção viável 
saindo de $v$ será combinação linear positiva destas direções, pelo seguinte argumento: para qualquer ponto $y \in P$, podemos escrever

$$
\begin{aligned}
y_{B} & =A_{B}^{-1} b-A_{B}^{-1} \tilde{A} \tilde{y} \\
& =v_{B}-\sum_{j \notin B} y_{j} A_{B}^{-1} A^{j} \\
& =v_{B}-\sum_{j \notin B} y_{j} d^{j} .
\end{aligned}
$$

logo as $n-m$ direções $d^{j}$ são todas as direções de arestas saindo de $v$. Isto conclui a prova.

Proposição 1.35 (Número de arestas saindo de $v$ ) Seja $P=\left\{x \in \mathbb{R}^{n} \mid A x \leqq b, x \geqq 0\right\}$ de dimensão $n$ e não-degenerado. Então a partir de qualquer vértice $v \in V(P)$ saem exatamente $n$ arestas.

Prova : Considere a representação canônica de $P$,

$$
P^{\prime}=\left\{\left(x, x^{r}\right)^{t} \in \mathbb{R}^{n} \times \mathbb{R}^{m} \mid A x+I x^{r}=b,\left(x, x^{r}\right)^{t} \geqq 0\right\}
$$

sabemos que $P^{\prime}$ é não-degenerado pois $P$ é não-degenerado. Pela proposição anterior, existem $(n+m)-m$ direções de arestas saindo de $(v, b-A v)$.

Falta então mostrar que as $n$ direções quando projetadas no espaço do poliedro original $P$, continuam sendo L.I. Isto é verdade pois a transformação linear que leva $P^{\prime} \mathrm{em} P$, dada por $\mathcal{M}\left(x, x^{r}\right)=x$, é inversível (com inversa dada por $\mathcal{M}^{-1}(x)=(x, b-A x)$ ), logo a independência linear será preservada, bem como a propriedade de qualquer direção viável saindo de $\mathcal{M}(v)$ ser combinação linear positiva destas direções projetadas.

Isto conclui a prova.

Proposição 1.36 (Representação por facetas) Seja $P \subset \mathbb{R}^{n}$ um poliedro de dimensão $n$, representado por um sistema $A x \leqq b$. Então este sistema pode ser reduzido apenas às desigualdades que definem facetas, isto é, se $\left\langle A_{\bar{\imath}}, x\right\rangle \leqq b_{\bar{\imath}}$ não define faceta, então

$$
\{x \mid A x \leqq b\}=\left\{x \mid\left\langle A_{j}, x\right\rangle \leqq b_{j}, \forall j \neq \bar{\imath}\right\}
$$

Prova : Como $P$ tem dimensão $n$, então $\operatorname{Int}(P) \neq \emptyset$. Seja $x^{0} \in \operatorname{Int}(P)$, tal que $x^{0} \in B\left(x^{0}, \varepsilon\right) \subset P$.

Suponha que o semi-espaço $\left\langle A_{\bar{\imath}}, x\right\rangle \leqq b_{\bar{\imath}}$ seja necessário ao sistema, isto é, suponha que exista algum elemento $x$ tal que $\left\langle A_{j}, x\right\rangle \leqq b_{j}, \forall j \neq \bar{\imath}$, mas $\left\langle A_{\bar{\imath}}, x\right\rangle>b_{\bar{\imath}}$. Como $P$ é convexo, então para todo $y \in B\left(x^{0}, \varepsilon\right)$ temos $\left\langle A_{\bar{\imath}}, y\right\rangle \leqq b_{\bar{\imath}}$, logo existe um $y^{\prime} \in[\{x, y\}]$ tal que $\left\langle A_{\bar{\imath}}, y\right\rangle=b_{\bar{\imath}}$. Porém $\left\{y^{\prime} \mid y \in B\left(x^{0}, \varepsilon\right)\right\} \subset P \cap\left\{\left\langle A_{\bar{\imath}}, x\right\rangle=b_{\bar{\imath}}\right\}$ tem dimensão $n-1$, logo $P \cap\left\{\left\langle A_{\bar{\imath}}, x\right\rangle=b_{\bar{\imath}}\right\}$ tem dimensão $n-1$; isto mostra que este semi-espaço define uma faceta, e conclui a prova. 


\subsection{Grafos e Fluxos}

Definição 1.37 (Grafos, Caminhos, Ciclos, Cortes e Árvores) Um grafo é um par $\mathcal{G}=(V, E)$ onde $V$ é um conjunto finito de nós e $E$ é um conjunto de pares $\{i, j\}$ chamados arcos, onde $i, j \in V, i \neq j$. Se os pares são ordenados, representamos os arcos por $(i, j)$ e dizemos que o grafo é dirigido. Todo grafo não-dirigido $\mathcal{G}=(V, E)$ pode ser transformado em um grafo dirigido $\mathcal{G}^{\prime}=\left(V, E^{\prime}\right)$ onde $E^{\prime}=\{(i, j),(j, i) \mid\{i, j\} \in E\}$.

Um caminho no grafo $\mathcal{G}=(V, E)$ é uma sequência $\left(i_{0}, e_{1}, i_{1}, e_{2}, \ldots, e_{k}, i_{k}\right)$ onde $i_{j} \in V, e_{j}=\left\{i_{j-1}, i_{j}\right\} \in E$ e $j \neq k \Longrightarrow i_{j} \neq i_{k}$. Se $\mathcal{G}$ é dirigido e $e_{j}=\left(i_{j-1}, i_{j}\right)$ então o caminho é dirigido.

Um ciclo é um caminho adicionado de uma aresta $e_{k+1}=\left\{i_{k}, i_{0}\right\}$ (ou $\left.e_{k+1}=\left(i_{k}, i_{0}\right)\right)$.

$U m$ subgrafo de um grafo $\mathcal{G}=(V, E)$ é um grafo $\mathcal{G}^{\prime}=\left(V^{\prime}, E^{\prime}\right)$ onde $V^{\prime} \subset V$ e $E^{\prime} \subset E$.

Um grafo é dito conexo se para cada par de nós $i, j$ existe um caminho entre $i$ e $j$.

As componentes conexas de um grafo são seus subgrafos conexos maximais.

Um corte de um grafo conexo $\mathcal{G}=(V, E)$ é uma partição $\left(S, S^{c}\right)$ do conjunto de nós, tal que $S, S^{c} \neq \emptyset$; associamos ao corte $\left(S, S^{c}\right)$ o conjunto $C=\left\{e=\{i, j\} \in E \mid i \in S, j \in S^{c}\right\}$, que também será chamado de corte.

Se $C$ é um corte associado a $\left(S, S^{c}\right)$, dizemos que $C$ é minimal se $\forall D \subset C, D \neq C$, temos que $(V, D)$ é conexo. Dado um subconjunto $S \subset V$, com $S \neq V$, indicaremos: $\delta(S)=\delta^{+}(S) \cup \delta^{-}(S)$, onde $\delta^{+}(S)=\{(i, j) \mid i \in S, j \notin S\}$ e $\delta^{-}(S)=\{(i, j) \mid i \notin S, j \in S\} . S e$ $S=\{v\}$ denotaremos $\delta(v)=\delta^{+}(v) \cup \delta^{-}(v)=\delta^{+}(S) \cup \delta^{-}(S)$.

Uma árvore em um grafo conexo $\mathcal{G}=(V, E)$ é um subgrafo $\mathcal{T}=\left(V, E^{\prime}\right.$ ) acíclico maximal (no número de arcos). Toda árvore tem \#V-1 arcos.

Os únicos grafos dirigidos com os quais trabalharemos são obtidos de grafos não-dirigidos segundo a definição acima.

Os nomes nó e arco substituirão neste trabalho os nomes mais comuns, vértice e aresta, para que não sejam confundidos com os conceitos já introduzidos para poliedros, na seção (1.1). Isto é necessário pois estudaremos vários poliedros construidos a partir de grafos, onde os quatro conceitos estarão presentes simultaneamente. 
Definição 1.38 (Fluxos, circulações) Seja $\mathcal{G}=(V, E)$ um grafo dirigido conexo, e se$j a b: V \longrightarrow \mathbb{R}$ tal que $\sum_{i \in V} b_{i}=0$. Um fluxo em $\mathcal{G}$ com respeito a $b$ é uma função $f: E \longrightarrow \mathbb{R}_{+}$tal que

$$
\sum_{(k, l) \in \delta^{+}(k)} f(k, l)-\sum_{(l, k) \in \delta^{-}(k)} f(l, k)=b_{k}, \forall k \in V
$$

Por conveniência representaremos esta função como um vetor $f=\left(f_{k l}\right)$, e a igualdade acima na forma matricial, $\mathcal{J} f=b$. Se $b_{k}=0, \forall k \in V$ dizemos que $f$ é uma circulação. Se $b_{i}=q, b_{j}=-q, b_{k}=0, \forall k \neq i, j$ dizemos que $f$ é um fluxo de $i$ a $j$ transportando $q$ (unidades de fluxo).

Proposição 1.39 (Decomposição de fluxos) Seja $\mathcal{G}$ um grafo dirigido conexo, $b$ um vetor tal que $b_{r}>0, b_{k} \leqq 0, \forall k \neq r, \sum_{k \in V} b_{k}=0$, e $f$ um fluxo com respeito a $b$. Então existem caminhos $\Pi_{j}^{r i}$ de $r$ a $i\left(\right.$ com $\left.b_{i}<0\right)$ e ciclos dirigidos $C^{k}$ tais que

$$
f=\sum_{i \neq r} \sum_{j=1}^{n_{i}} \alpha_{j}^{i} \Pi_{j}^{r i}+\sum_{k=1}^{l} \beta_{k} C^{k},
$$

onde $\alpha_{j}^{i}, \beta_{k} \geqq 0, \sum_{j=1}^{n_{i}} \alpha_{j}^{i}=-b_{i}, \forall i \neq r$. Além disso $\sum_{i \neq r} n_{i}+l \leqq \# V+\# E, l \leqq \# E$.

Prova : A prova é algorítmica: seja $i_{0}$ um nó qualquer tal que $b_{i_{0}}<0$. Pelas equações (1.38), existe algum arco $\left(i_{1}, i_{0}\right)$ tal que $f_{i_{1} i_{0}}>0$. Se $i_{1}=r$, paramos. Caso contrário, $b_{i_{1}} \leqq 0$, e existe algum arco $\left(i_{2}, i_{1}\right)$ tal que $f_{i_{2} i_{1}}>0$. Repetindo o argumento, em no máximo \#V passos teremos um nó $i_{k}$ tal que ou $i_{k}=r$ ou $i_{k}$ já foi visitado anteriormente. Note que o fluxo ao longo dos arcos $\left\{\left(i_{j}, i_{j-1}\right)\right\}_{j=1}^{k}$ é estritamente positivo, por construção.

Se $i_{k}=r$, redefinimos $f_{i j} \longleftarrow f_{i j}-\delta$ ao longo do caminho orientado $\left(i_{0}, i_{1}, \ldots, i_{k}=r\right)$, onde $\delta=\min \left\{-b_{i_{0}},-b_{i_{k}}, f_{i_{l+1} i_{l}} \mid l=0, \ldots, k-1\right\}>0$, e $b_{i_{0}} \longleftarrow b_{i_{0}}+\delta, b_{r} \longleftarrow b_{r}-\delta$. Se $i_{k}$ já foi visitado, temos um ciclo dirigido. Redefinimos $f_{i j} \longleftarrow f_{i j}-\delta$ ao longo do ciclo $\left(i_{p}, i_{p+1}, \ldots, i_{k}\right)$, onde $\delta=\min \left\{f_{i_{l+1} i_{l}} \mid l=p, \ldots, k-1\right\}>0$.

É claro que estes fluxos redefinidos são fluxos com respeito ao novo $b$. Além disso a cada caminho encontrado temos ou um arco cujo fluxo é zerado, ou algum $b_{k}$ é zerado; e a cada ciclo encontrado algum arco tem seu fluxo zerado.

Por esta razão após no máximo $\# E+\# V$ passos teremos que $b_{k}=0, \forall k \in V$; poderíamos pensar que os fluxos $f_{i j}$ poderiam ser todos zerados antes dos $b_{k}$, mas por (1.38) temos que $f=0 \Longrightarrow b=0$. Neste ponto, podemos escolher um arco com fluxo positivo e repetir o argumento anterior para o caso do ciclo, até que $f=0$. Sejam $\Pi_{j}^{r i}, j=1, \ldots, n_{i}$ todos os caminhos de $r$ a $i$ detectados, e $\alpha_{j}^{i}$ os valores de $\delta$ associados, para $i \neq k$; e sejam $C^{k}, k=1, \ldots, l$ os ciclos dirigidos, e $\beta_{j}$ os valores de $\delta$ associados. 
Então temos que

$$
f^{\text {final }}=0=f^{\text {inicial }}-\sum_{i \neq r} \sum_{j=1}^{n_{i}} \alpha_{j}^{i} \Pi_{j}^{r i}-\sum_{k=1}^{l} \beta_{k} C^{k},
$$

e $b_{i}^{\text {final }}=0=b_{i}^{\text {inicial }}-\sum_{j=1}^{n_{i}} \alpha_{j}^{i}, \forall i \neq r$. Além disso a cada ciclo ou caminho encontrado temos ou um arco cujo fluxo é zerado, ou um $b_{k}$ zerado. Isso mostra que $\sum_{i \neq r} n_{i}+l \leqq \# V+\# E$. E como a cada ciclo detectado pelo menos um $f_{i j}$ é zerado, temos $l \leqq \# E$.

Proposição 1.40 (Existência de fluxo sob restrição de capacidade nos arcos) Seja $\mathcal{G}$ um grafo dirigido conexo, $u \geqq 0$ um vetor de capacidades nos arcos de $\mathcal{G}$, e b um vetor tal que $b_{r}>0, b_{k} \leqq 0, \forall k \neq r, e \sum_{k \in V} b_{k}=0$. Então existe um fluxo com respeito a $b$ satisfazendo $f_{i j} \leqq u_{i j}, \forall(i, j) \in E$ se e somente se para todo subconjunto $S \subset V$ tal que $r \in S$, temos que $\sum_{i \in S} b_{i} \leqq \sum_{(i, j) \in \delta^{+}(S)} u_{i j}$.

Prova : A condição apresentada é certamente necessária, pois se $f$ é um fluxo viável com respeito à $b$ e às restrições de capacidade, então dado $S \subset V$ com $r \in S$, podemos somar as equações em (1.38) para $i \in S$, obtendo $\sum_{(i, j) \in \delta^{+}(S)} f_{i j}-\sum_{(j, i) \in \delta^{-}(S)} f_{j i}=\sum_{i \in S} b_{i}$, logo

$$
\sum_{(i, j) \in \delta^{+}(S)} u_{i j}+0 \geqq \sum_{(i, j) \in \delta^{+}(S)} f_{i j}-\sum_{(j, i) \in \delta^{-}(S)} f_{j i}=\sum_{i \in S} b_{i} .
$$

Por outro lado, considere que para todo subconjunto $S \subset V$ tal que $r \in S$, temos que $\sum_{i \in S} b_{i} \leqq \sum_{(i, j) \in \delta^{+}(S)} u_{i j}$. Considere o grafo obtido pela inclusão de todos os arcos $\{(i, r) \mid i \in \overline{\bar{V}} \backslash\{r\}\}$ que apontam para a origem $r$. Definiremos limitantes inferiores e superiores para o fluxo nestes arcos como $l_{i r}=u_{i r}=-b_{i} \geqq 0$, e para os demais arcos do grafo teremos $l_{i j}=0$ e $u_{i j}$ como no enunciado. É então imediato que se $S \subset V$ é tal que $r \in S$, então

$$
\sum_{(i, j) \in \delta^{+}(S)} u_{i j}-\sum_{(j, i) \in \delta^{-}(S)} l_{j i} \geqq 0,
$$

pois

$$
\sum_{(i, j) \in \delta^{+}(S)} u_{i j}-\sum_{(j, i) \in \delta^{-}(S)} l_{j i}=\sum_{(i, j) \in \delta^{+}(S)} u_{i j}+\sum_{i \notin S} b_{i} \geqq \sum_{i \in S} b_{i}+\sum_{i \notin S} b_{i}=0 .
$$

Iremos agora mostrar que existe uma circulação viável neste novo grafo, de onde teremos automaticamente um fluxo com respeito a $b$ satisfazendo as restrições de capacidade. Considere a circulação inicial $f=0$; esta circulação satisfaz todas as restrições de capacidade, e viola algumas restrições $f_{i j} \geqq l_{i j}$. Construiremos uma cirulação viável algoritmicamente. Para tanto, manteremos durante o algoritmo a validade da seguinte propriedade: toda vez que $f_{i j}>l_{i j}$ teremos que $f_{j i} \leqq l_{j i}$. Esta propriedade (1) poderá 
sempre ser mantida, pois toda vez que $f_{i j}>l_{i j}$ e $f_{j i}>l_{j i}$ simultaneamente poderemos subtrair $\delta=\min \left\{f_{i j}-l_{i j}, f_{j i}-l_{j i}\right\}>0$ tanto de $f_{i j}$ quanto de $f_{j i}$, sem perder nenhuma das restrições já satisfeitas.

Seja $(p, r)$ um arco tal que $f_{p r}<l_{p r}$ (note que estes são os únicos arcos que poderiam estar violando a restrição $f_{i j} \geqq l_{i j}$ ). Afirmamos que existe um caminho dirigido de $r$ até $p$ através de arcos não-saturados, isto é, ao longo do qual todas as restrições de capacidade estão folgadas $\left(f_{i j}<u_{i j}\right)$. Suponha que isto não seja verdade. Seja $S$ o conjunto dos nós alcançaveis a partir de $r$ por caminhos dirigidos não-saturados. Por construção, temos que $r \in S$ e $f_{i j}=u_{i j}>l_{i j}=0, \forall(i, j) \in \delta^{+}(S)$. Pela propriedade (1) teremos que $f_{j i} \leqq l_{j i}, \forall(j, i) \in \delta^{-}(S)$, que juntamente com $f_{p r}<l_{p r}$ e $(p, r) \in \delta^{-}(S)$ mostra que

$$
\sum_{(i, j) \in \delta^{+}(S)} u_{i j}-\sum_{(j, i) \in \delta^{-}(S)} l_{j i}<\sum_{(i, j) \in \delta^{+}(S)} f_{i j}-\sum_{(j, i) \in \delta^{-}(S)} f_{j i}=0
$$

uma contradição. Isso mostra que deverá existir um caminho dirigido de $r$ até $p$ através de arcos não-saturados. Iremos então aumentar o fluxo transportado através do ciclo $C$ formado por este caminho e o arco $(p, r)$ de uma quantidade $\delta=\min \left\{u_{i j}-f_{i j}+f_{j i}-l_{j i} \mid(i, j) \in C\right\}>0$. Se for necessário, aplicaremos o procedimento descrito acima para preservar a propriedade (1). A nova circulação obtida satisfaz todas as restrições de capacidade; além disto tem pelo menos um arco saturado a mais do que a anterior. Repetindo o argumento teremos, em no máximo \#E passos, uma circulação satisfazendo todas as restrições. Retirando os arcos $(i, r)$, para $i \in V \backslash\{r\}$ teremos um fluxo com respeito a $b$ satisfazendo todas as restrições de capacidade.

A proposição (1.40) é um lema que precede um teorema bem mais forte, conhecido como max-flow=min-cut [Ford \& Fulkerson 62]; para este texto, a proposição acima será suficiente.

Um estudo mais abrangente sobre fluxos em redes pode ser encontrado nos textos [Ahuja, Magnanti \& Orlin 93] e [Bazaraa \& Jarvis 77]. 


\section{Capítulo 2}

\section{O Problema Motivador}

Neste capítulo faremos uma breve exposição da rede LARC, motivadora do trabalho desenvolvido por [Humes 88], que é o ponto de partida desta dissertação. A partir dos importantes resultados de concavidade da função $D^{*}$ (seção (2.3)), discutiremos dois dos principais métodos em Programação Côncava, e abordaremos as dificuldades de utilização imediata dos mesmos para o problema (CFA), definido na seção (2.2.3).

\subsection{A Rede LARC}

Na década de 80, foram projetadas no Brasil algumas redes experimentais no meio acadêmico, inspiradas e amparadas pela pesquisa desenvolvida em função da rede ARPA (Departamento de Defesa dos Estados Unidos, 1969).

Uma destas foi a rede LARC (Laboratório Nacional de Redes de Computadores), cujo projeto inicial era interligar universidades e centros de pesquisa em Belo Horizonte, Brasília, Campinas, Campina Grande, Porto Alegre, Recife, Rio de Janeiro, São José dos Campos e São Paulo. Esta seria uma rede de chaveamento por pacotes, tendo por uso principal a troca de mensagens.

A abordagem padrão para projeto considera didaticamente a existência de três níveis de projeto: projeto topológico, projeto do roteamento e designação de capacidades. O projeto do roteamento e a designação de capacidades são intrinsicamente interligados, e dependem diretamente do projeto topológico.

Estes três níveis de projeto são explicados a seguir:

1. O projeto topológico consiste em determinar a localização dos nós da rede, bem como estabelecer um esquema de interconexão entre estes nós, isto é, estabelecer quais pares de nós estariam diretamente interligados por um canal de comunicação. Os nós podem corresponder a hosts, bem como a localidades unicamente dedicadas ao roteamento de mensagens na rede. É natural que no caso geral não se pensasse em interligar diretamente todos os pares de nós, pois tal solução seria economicamente inviável.

Tal projeto deveria levar em consideração fatores de segurança, como por exemplo a preservação da conectividade da rede na eventual falha de um dos canais de comunicação (biconectividade), além de fatores como economia de recursos e aumento da performance da 
rede. Devemos lembrar que a cada diferente topologia estão associadas várias possibilidades de roteamento das mensagens para os diversos pares de nós, além de várias velocidades de transmissão nos canais de comunicação utilizados. O problema global, assim colocado, é bastante difícil, pela presença de um problema combinatório (projeto topológico) juntamente com o problema do roteamento e da designação de capacidades, que em geral é não-linear e não-convexo.

No presente estudo estaremos trabalhando sob uma topologia fixada, dada juntamente com a descrição do problema. Esta topologia dada será chamada de topologia maximal, pois está associada aos arcos que podem ser usados como canais de comunicação (algumas soluções podem não usar todos os arcos disponíveis).

2. Outro aspecto do projeto é decidir a capacidade de transmissão de cada um dos canais de comunicação da rede. Estas capacidades deverão acomodar os fluxos de transmissão, ao mesmo tempo minimizando o custo total de instalação (proporcional às capacidades dos canais) e o retardo médio de transmissão das mensagens. Este último depende da relação entre os fluxos e as capacidades em cada canal e, como veremos adiante, tende a infinito sempre que qualquer dos fluxos em algum canal tende à capacidade instalada no mesmo. Isso fará com que uma das restrições de viabilidade seja

$$
\text { (fluxo no canal } i)<(\text { capacidade do canal } i) \text {, }
$$

diferentemente das usuais restrições de capacidades nos fluxos de um grafo, que não utilizam a desigualdade estrita. É importante mencionar que o conjunto de capacidades disponíveis (no mercado) para aquisição dos canais é finito, usualmente formado por múltiplos de uma certa capacidade mínima (por exemplo, $C=\{0,1.2,2.4,4.8,9.6,14.4,28.8 \mathrm{kbits} / \mathrm{seg}\}$ ), mas no nosso trabalho trabalharemos com a chamada relaxação contínua, isto é, assumiremos que o conjunto de capacidades disponíveis é $C=\mathbb{R}^{+}$.

3. A decisão dos fluxos em cada canal traz consigo uma decisão do roteamento das mensagens na rede. Assumiremos que este roteamento é fixo para cada solução, isto é, em uma dada solução a cada par de nós comunicantes (origem, destino) existirá um conjunto de caminhos no grafo associado de origem até destino e um conjunto de pesos associados aos caminhos de forma a determinar unicamente o trajeto (possivelmente distribuido) das mensagens entre este par de nós. Os valores de fluxo utilizados representarão valores médios, e dependerão portanto da demanda média de comunicação entre os diversos pares de nós. Como não existe um custo financeiro associado ao transporte propriamente dito dos fluxos, o projeto destes estará associado principalmente ao retardo médio das mensagens na rede. A modelagem dos fluxos seguirá de perto o que já foi dito na seção (1.4).

A alta inter-relação entre os dois últimos níveis de projeto nos leva a estudá-los conjuntamente (designação de fluxos e capacidades).

Devemos observar, no entanto, que o projeto topológico poderia ser integrado de forma implícita ao problema da designação dos fluxos e capacidades, ao assumirmos que a topologia disponível é a do grafo completo. Neste problema, qualquer solução para uma subtopologia será também uma solução para a topologia do grafo completo, com mesmo custo e mesmo retardo associados. Tal possibilidade não nos parece porém eficiente, por pelo menos três razões. A primeira 
está diretamente relacionada com o número de vértices associado ao problema de designação de fluxos e capacidades (ver capítulo (3)); este número em geral cresce exponencialmente em função do número de arcos disponíveis. A outra razão é a deste tratamento não utilizar nenhuma estrutura associada ao conjunto de topologias possíveis; além disto, esta abordagem não leva também em conta fatores de segurança como biconectividade.

\subsection{O (CFA) e suas Formulações}

Estamos então aptos a introduzir o problema de designação de fluxos e capacidades (com a topologia maximal dada):

\subsubsection{Descrição Informal}

Considere uma rede de computadores com $n$ nós rotulados de 1 a $n$, para a qual se conhecem valores de demanda média de comunicação entre os pares $(r, s)$ de nós, $q_{r s}>0$ (medidas, por exemplo, em kbits/seg). A hipótese de $q_{r s}$ não poder anular-se é forte, mas fundamental para garantir a não-degenerescência do poliedro $\vec{F}^{r}$ que será estudado na seção (3.2).

O problema de designação de fluxos e capacidades (CFA - do inglês Capacity and Flow Assignment) consiste em encontrar roteamentos para as mensagens originadas em $r$ e destinadas a $s$ (para todos os pares $(r, s)$ ), e capacidades para os canais de interconexão da rede, de forma a minimizar o retardo médio das mensagens e o custo de projeto.

Trata-se de um problema bicritério, de onde a noção de Pareto Eficiência surge naturalmente:

Definição 2.1 (Pareto Eficiência) Seja $X$ um conjunto e $g: X \longrightarrow \mathbb{R}^{q}$ uma função representando $q$ critérios de minimização. Um ponto $A$ é dito eficiente no sentido de Pareto se não existe outro ponto $B$ tal que $g(B) \leq g(A)$, isto é, que seja no mínimo tão bom quanto $A$ em relação a todos os critérios, mas estritamente melhor em pelo menos um deles.

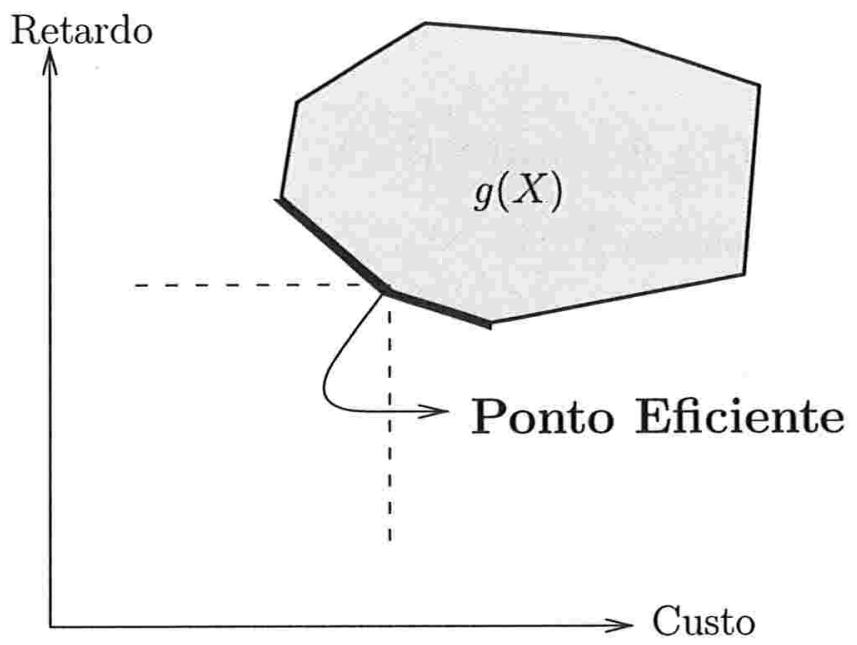


No nosso caso, um projeto de rede $A$ é Pareto Eficiente se não existir um outro projeto $B$ com retardo médio menor e custo pelo menos tão baixo quanto os de $\mathrm{A}$, ou ainda com custo menor e retardo médio pelo menos tão baixo quanto os de A, como os pontos mais escuros na figura acima.

Esta noção sugere a decomposição do problema original em dois problemas monocritério que serão definidos na seção (2.2.3), cada um deles associado a um dos critérios, e com a seguinte propriedade: se $x$ é solução de um dos dois problemas monocritério, então $x$ será um projeto eficiente para o problema original.

\subsubsection{Modelagem do Problema}

A topologia da rede pode ser convenientemente representada por um grafo conexo dirigido $\vec{G}=(V, \vec{E})$ onde $V=\{1,2, \ldots, n\}$ é o conjunto de localidades que serão conectadas e $\vec{E}$ é o conjunto de arcos $(i, j)$ representando os canais de comunicação. Para modelar os chamados canais full-duplex, que transmitem sinais nos dois sentidos, o grafo terá a propriedade de que a todo arco $(i, j)$ correponderá o arco reverso, $(j, i)$; estes dois arcos do grafo correspondem na realidade a apenas um canal de comunicação. Freqüentemente utilizaremos o grafo não-dirigido $G=(V, E)$ obtido de $\vec{G}$ pela identificação das arestas $(i, j)$ e $(j, i)$. Para simplificar a notação, representaremos $\vec{E}=\{1,2, \ldots, 2 m\}$ e $E=\{1,2, \ldots, m\}$.

Dadas as demandas de comunicação $q_{r s}>0$ entre cada par $(r, s)$ de nós da rede, pode-se modelar os fluxos a partir do conjunto de variáveis $f_{k l}^{r s} \geqq 0$ (medidos, por exemplo, em kbits/seg) correspondentes ao fluxo médio de transmissão gerada em $r$ e destinada a $s$, passando pelo arco $(k, l)$ no sentido de $k$ para $l$. Estas variáveis estão portanto modelando os fluxos no grafo dirigido.

Sob a hipótese de que todas as mensagens geradas internamente à rede (tais como retransmissões e pacotes de controle) podem ser tratadas como um overhead proporcional às demandas consideradas (estando já contabilizadas em $q_{r s}$ ), os fluxos correspondentes a cada par $(r, s)$ devem satisfazer, como visto na seção (1.4),

$$
\begin{gathered}
\sum_{l \in \delta^{+}(k)} f_{k l}^{r s}-\sum_{l \in \delta^{-}(k)} f_{l k}^{r s}=0, \forall k \in V /\{r, s\} \\
\sum_{l \in \delta^{+}(r)} f_{r l}^{r s}-\sum_{l \in \delta^{-}(r)} f_{l r}^{r s}=\sum_{l \in \delta^{-}(s)} f_{l s}^{r s}-\sum_{l \in \delta^{+}(s)} f_{s l}^{r s}=q_{r s}
\end{gathered}
$$

$\mathrm{e}$

$$
f_{k l}^{r s} \geqq 0, \forall(r, s) \in V \times V, \forall(k, l) \in \vec{E}
$$

Estas equações definem o poliedro $\vec{F}^{r s}$ :

Definição 2.2 (Poliedro de fluxos dirigidos de $r$ a $s: \vec{F}^{r s}$ )

$$
\vec{F}^{r s}=\left\{\left(f_{k l}^{r s}\right) \mid(1,(2)) \text { e (3) são satisfeitas }\right\}
$$

Para um par $(r, s)$ fixado, o conjunto das equações (1) e (2) acima é L.D., pois qualquer uma das equações em (1) ou (2) pode ser escrita como a soma das demais (trocando-se o sinal). 
Veremos adiante que fluxos que contém ciclos positivos não serão candidatos a pontos Paretoeficientes. Esta afirmação é provada utilizando a caracterização do cone associado a $\vec{F}^{r s}$ (seção (3.3)) e a hipótese de custos estritamente crescentes, e corresponde à intuição natural.

Para cada origem $r$ o poliedro $\vec{F}^{r}$ dos fluxos gerados em $r$ e destinados a qualquer outro nó é definido por

Definição 2.3 (Poliedro de fluxos dirigidos a partir de $r: \vec{F}^{r}$ )

$$
\vec{F}^{r}=\sum_{s \neq r} \vec{F}^{r s}=\left\{\left(f_{k l}^{r}\right)=\sum_{s \neq r}\left(f_{k l}^{r s}\right) \mid\left(f_{k l}^{r s}\right) \in \vec{F}^{r s}, \forall s \neq r, s \in V\right\}
$$

O poliedro de fluxos (dirigidos) multicomodidade é definido então por

Definição 2.4 (Poliedro de fluxos dirigidos multicomodidade: $\overrightarrow{\mathcal{F}}$ )

$$
\overrightarrow{\mathcal{F}}=\sum_{r \in V} \vec{F}^{r}=\left\{\left(f_{k l}\right)=\sum_{s \neq r}\left(f_{k l}^{r}\right) \mid\left(f_{k l}^{r}\right) \in \vec{F}^{r}, \forall r \in V\right\}
$$

O termo comodidade é utilizado para distingüir a diferença natural entre mensagens associadas a diferentes pares (origem,destino); estas mensagens não podem ser tratadas igualmente, embora sejam transmitidas através dos mesmos canais de comunicação (não existem automaticamente canais reservados somente a um determinado par (origem,destino)).

Note que a descrição completa do roteamento das mensagens é equivalente ao conhecimento do valor de todas as variáveis $f_{k l}^{r s}$. O poliedro $\overrightarrow{\mathcal{F}}$ sozinho não contém toda a informação relativa a este roteamento, pois a cada arco temos a soma de fluxos resultante de várias origens e destinos.

Como veremos adiante, tanto a função objetivo quanto as restrições para a designação de capacidades dependem apenas dos fluxos totais nos canais de comunicação; para estas a origem e o destino, bem como a orientação dos fluxos é irrelevante. Por esta razão serão também importantes para o nosso estudo os poliedros obtidos pela transformação linear que soma todos os fluxos que passam pelo mesmo canal de comunicação; lembremos que este foi representado por dois arcos orientados com as mesmas extremidades, mas sentidos opostos. Utilizaremos o termo projeção nos referindo a esta transformação linear. Os poliedros projetados são então definidos a seguir.

Definição 2.5 (Poliedro de fluxos de $r$ a $s: F^{r s}$ )

$$
F^{r s}=\left\{f=\left(f_{1}, f_{2}, \ldots, f_{m}\right) \mid f_{e}=f_{k l}^{r s}+f_{l k}^{r s} \text { onde } e=\{k, l\} e\left(f_{k l}^{r s}\right) \in \overrightarrow{F^{r} s}\right\} .
$$

Definição 2.6 (Poliedro de fluxos a partir de $r: F^{r}$ )

$$
F^{r}=\left\{f=\left(f_{1}, f_{2}, \ldots, f_{m}\right) \mid f_{e}=f_{k l}^{r}+f_{l k}^{r} \text { onde } e=\{k, l\} e\left(f_{k l}^{r}\right) \in \vec{F}^{r}\right\} .
$$


Definição 2.7 (Poliedro de fluxos multicomodidade: $\mathcal{F}$ )

$$
\mathcal{F}=\left\{f=\left(f_{1}, f_{2}, \ldots, f_{m}\right) \mid f_{e}=f_{k l}+f_{l k} \text { onde } e=\{k, l\} e\left(f_{k l}\right) \in \overrightarrow{\mathcal{F}}\right\} .
$$

É importante notar que para quaisquer $q_{r s}>0$ os poliedros definidos acima são sempre nãovazios, porque o grafo é conexo.

Para a modelagem das capacidades foi assumida a chamada relaxação contínua para o problema, que consiste em adotar como conjunto de capacidades viáveis todo o $\mathbb{R}_{+}$. Há portanto um valor $c_{e}$ associado a cada canal $e \in E$ e o custo de um canal com esta capacidade é dado por uma função $d_{e}\left(c_{e}\right)$ (uma para cada aresta do grafo, pois o custo não depende exclusivamente da capacidade do canal, mas também de sua extensão, do tipo da região que ele vai atravessar, etc). Assumindo que o único custo envolvido no projeto é o custo total dos canais de comunicação que devem ser instalados, o custo do projeto da rede será dado por

$$
D(c)=\sum_{e \in E} d_{e}\left(c_{e}\right)
$$

Trata-se de uma hipótese simplificadora, mas justificada pela visão abstrata da rede como um meio de troca de informações (os demais recursos teriam portanto um custo pouco ou não controlável).

Algumas hipóteses naturais sobre as funções $d_{e}(\cdot)$ são:

1. $d_{e}(0)=0, \forall e$ (não há custo envolvido em não se instalar um canal),

2. $d_{e}(\cdot)$ é contínua e estritamente crescente (canais mais rápidos são mais caros), e

3. $d_{e}(\cdot)$ é côncava (ou estritamente côncava);

Esta última hipótese está relacionada a fatores de escala (um exemplo simples: já que dois canais com capacidade $x$ em paralelo fornecem a mesma taxa de transmissão de um só de capacidade $2 x$, é natural que custo $(2 x) \leqq 2 * \operatorname{custo}(x))$. No caso da EMBRATEL é usado o modelo

$$
d_{e}\left(c_{e}\right)=l_{e} c_{e}^{\alpha}
$$

onde $\alpha \approx 0.56 \in(0,1]$ e $l_{e}>0$ depende da distância entre as localidades a serem interligadas. Este é o modelo que adotaremos no restante do trabalho:

Definição 2.8 (Função custo para o (CFA): $D(\cdot)$ )

$$
D(c)=\sum_{e \in E} l_{e} c_{e}^{\alpha}
$$

onde $l_{e}>0$, e $\alpha \in(0,1]$.

Note que a função acima definida satisfaz as três hipóteses mencionadas, e $D(\cdot)$ é estritamente côncava sempre que $\alpha<1$. Faremos constante referência aos casos de custos lineares (isto é, $\alpha=1$ ) e custos estritamente côncavos (isto é, $\alpha<1$ ). 
A modelagem do retardo em função de fluxos e capacidades é certamente o ponto mais trabalhoso da formulação do problema. Será aceito por modelo de trabalho uma rede de filas M/M/1 com roteamento dado, onde cada nó possui um buffer para armazenar as mensagens que utilizam aquele nó como ponto de passagem, esperando para a retransmissão. Esta última característica é compatível com a técnica de store-and-forward, que descrevemos brevemente a seguir.

A técnica de store-and-forward consiste em dotar cada um dos nós da rede de um buffer para armazenamento de mensagens e de um software processador da distribuição das mensagens. Uma mensagem que chega ao nó $i$ com um destino diferente de $i$, e que deve ser roteada através do canal $(i, k)$, poderá ter que esperar, no buffer, até que aquele canal esteja desocupado. O processador organizará portanto a entrada e saída das mensagens neste buffer, observando a disponibilidade dos canais e o roteamento (dado) das mensagens.

Uma condição importante para o dimensionamento dos buffers é de que o tamanho das mensagens não exceda um certo limitante superior. Note que por mensagem entendemos não só o conteúdo propriamente transmitido, mas também informações relativas ao roteamento e aos protocolos de segurança (ver por exemplo [Tanenbaum 89]) utilizados pela rede, na forma de $c a-$ beçalhos e caudas. O nome usual para este tipo de mensagem com cabeçalhos e limite no tamanho é pacote. Neste texto utilizaremos o termo mensagem com estas restrições, isto é, significando pacote. Freqüentemente trataremos a informação no canal de comunicação como um stream de bits.

Outra técnica utilizada para comunicação é a de chaveamento por circuito, ou circuito virtual. Neste caso, a cada pedido de comunicação com origem em $i$ e destinada a $j$ é alocado um caminho de canais desde $i$ até $j$, que ficam reservados para a comunicação entre este par somente, até que um dos dois nós emita um sinal de desconexão, liberando os canais para o uso do restante da rede. Esta técnica é bastante eficiente para a transferência de arquivos de grande porte, pois uma vez estabelecida a conexão, os canais serão utilizados ininterruptamente com a maior taxa de transmissão que for permitida. Porém em situações de comunicações por picos (bursty mode), os tempos para o estabelecimento e liberação do circuito virtual podem tornar-se um gargalo em relação ao tempo das transmissões propriamente ditas.

Algumas redes utilizam as duas técnicas, passando a operar com circuitos virtuais em determinados horários para a transmissão de arquivos de porte.

Nosso trabalho assumirá uma rede de filas $\mathrm{M} / \mathrm{M} / 1 \mathrm{com}$ chaveamento por pacotes, utilizando a técnica de store-and-forward. Neste modelo temos

$$
\begin{aligned}
& \text { Definição } 2.9 \text { (Retardo no arco e retardo médio para o (CFA)) Se } f \in \mathcal{F} \text {, e c } \in \mathbb{R}_{+}^{m} \\
& \text { satisfaz } c_{I(f)}>f_{I(f)} \text {, o retardo } t_{e} \text { no canal } e \in E \text { é dado por } \\
& \qquad t_{e}\left(f_{e}, c_{e}\right)= \begin{cases}\frac{f_{e}}{c_{e}-f_{e}}, & \text { se } f_{e} \neq 0 \\
0, & \text { se } f_{e}=0\end{cases} \\
& \begin{array}{l}
\text { e o retardo médio é } \\
\text { onde } \hat{q}=\sum_{(r, s) \in V \times V} q_{r s} .
\end{array}
\end{aligned}
$$


Este modelo foi extensamente estudado na literatura, e optamos por não fazer uma derivação muito detalhada aqui. Esta derivação pode ser encontrada em [Kleinrock 76], [Humes 88] e [Gerla 73], utilizando resultados teóricos de [Jackson 57].

Uma condição elementar que relaciona fluxos e capacidades é $c_{I(f)}>f_{I(f)}$, pois do contrário a quantidade de informação armazenada nos nós, esperando para ser retransmitida, cresceria indefinidamente, como é facilmente visto na definição (2.9). A restrição usual de capacidades em grafos $\left(f_{e} \leqq c_{e}\right)$ não prevê efeitos como o congestionamento das mensagens, que são fundamentais neste modelo.

\subsubsection{O (CFA) clássico}

A partir da modelagem apresentada na seção anterior, podemos enunciar a primeira versão do problema de designação de fluxos e capacidades (Capacity and Flow Assignment = CFA) assim:

$$
\left\{\begin{aligned}
& \text { Dado } G=(V, A) \text { e } q: V^{2} \rightarrow \mathbb{R}_{+} \\
& \text {"minimizar" }(T(f, c), D(c))^{t} \\
& \text { sujeito a } f \in \mathcal{F} \\
& c_{I(f)}>f_{I(f)} \\
& c_{e}=0, \forall e \notin I(f)
\end{aligned}\right.
$$

Um grande problema com esta formulação é a interpretação do termo minimizar quando aplicado a dois critérios independentes, pois o $\mathbb{R}^{2}$ não é linearmente ordenado.

Podemos então simplesmente procurar pontos pareto-eficientes. Neste caso existirão vários pontos pareto-eficientes, para os quais o critério $(T(f, c), D(c))^{t}$ não fornece nenhuma razão para preferir um ou outro.

Outra possibilidade é atribuirmos pesos positivos aos diversos critérios, transformando o problema multicritério em monocritério, através de uma combinação linear. No presente caso, poderíamos considerar minimizar a função $\alpha T(f, c)+\beta D(c)$ onde $\alpha$ e $\beta$ são constantes positivas.

Em [Lin 76], são obtidas diversas relações entre as duas abordagens; é exibido também um método de decomposição de um problema genérico com $k>1$ critérios em $k$ famílias de problemas monocritério, de forma que se $x^{*}$ é solução dos $k$ problemas monocritério então $x^{*}$ é eficiente no problema original. Embora este método não possa ser implementado de maneira direta na prática (pois os $k$ problemas se escrevem em função da solução ótima $x^{*}$ ), pode-se aliar esta descrição a um método iterativo que a partir de uma solução viável, a cada passo melhora a solução em relação a um dos critérios. O (CFA) é passível desta decomposição; um dos problemas terá o custo $D(\cdot)$ como função objetivo e uma restrição de retardo (médio) máximo, enquanto o outro terá o retardo médio $T(\cdot, \cdot)$ como função objetivo e uma restrição de custo máximo. Os problemas são formulados da seguinte maneira:

$$
P D\left(T_{\mathrm{MAX}}\right) \begin{cases}\text { minimizar } & D(c) \\ s . a . & T(f, c) \leqq T_{\mathrm{MAX}} \\ & c_{I(f)}>f_{I(f)} \\ & c_{e}=0, \forall e \notin I(f) \\ & (f, c) \in \mathcal{F} \times \mathbb{R}_{+}^{m}\end{cases}
$$




$$
P T\left(D_{\text {MAX }}\right) \begin{cases}\text { minimizar } & T(f, c) \\ s . a . & D(c) \leqq D_{\text {MAX }} \\ & c_{I(f)>f_{I(f)}} \\ & c_{e}=0, \forall e \notin I(f) \\ & (f, c) \in \mathcal{F} \times \mathbb{R}_{+}^{m}\end{cases}
$$

Inicialmente nota-se que $P D\left(T_{\text {MAX }}\right)$ é viável para qualquer $T_{\text {MAX }}>0$ e $P T\left(D_{\text {MAX }}\right)$ é viável para todo $D_{\mathrm{MAX}}>D_{0}=\inf \{D(f) / f \in \mathcal{F}\}$. A primeira afirmação é clara pois, para todo fluxo $f \in \mathcal{F}$ viável, existe uma capacidade suficientemente grande que garanta a restrição de retardo médio; isto é, existe um $M \in \mathbb{R}_{+}(M>1)$ e um vetor $c=M f$ de tal forma que

$$
T(f, c)=\frac{1}{\hat{q}} \sum_{e \in I(f)} \frac{f_{e}}{c_{e}-f_{e}}=\frac{m}{(M-1) \hat{q}} \leqq T_{\mathrm{MAX}}
$$

Para a segunda afirmação, segue da definição de $D_{0}$ e $D_{\text {MAX }}$ e do fato de $\mathcal{F} \neq \emptyset$ que existe um $f \in \mathcal{F}$ tal que $D_{0} \leqq D(f)<D_{\text {MAX }}$, e pela continuidade e monotonicidade de $D(\cdot)$ existe um $\varepsilon>0$ de tal forma que $c=(1+\varepsilon) f$ satisfaz

$$
D(f)<D(c)<D_{\mathrm{MAX}}
$$

Além disso os dois problemas, quando viáveis, tem solução ótima (a demonstração encontra-se em [Humes 88]), e mais ainda, valem as seguintes conclusões:

1. $\forall T_{\text {MAX }}>0,\left(T_{\text {MAX }}, D^{*}\left(T_{\text {MAX }}\right)\right)$ corresponde a um projeto eficiente, onde $D^{*}\left(T_{\mathrm{MAX}}\right)$ é o valor ótimo de $P D\left(T_{\mathrm{MAX}}\right)$, e

2. $\forall D_{\text {MAX }}>D_{0},\left(T^{*}\left(D_{\text {MAX }}\right), D_{\text {MAX }}\right)$ corresponde a um projeto eficiente, onde $T^{*}\left(D_{\mathrm{MAX}}\right)$ é o valor ótimo de $P T\left(D_{\mathrm{MAX}}\right)$.

Iremos trabalhar especificamente com o problema $P D\left(T_{\mathrm{MAX}}\right)$, para o qual podemos obter uma formulação, cuja função objetivo depende apenas dos fluxos, e corresponde a resolver um problema sobre as variáveis $c_{e}$, como veremos a seguir.

\subsection{A Função $D^{*}(\cdot)$}

Por conveniência, nesta seção todos os fluxos considerados estarão no $\mathbb{R}_{+}^{m}$ (e não somente em $\mathcal{F}$ ). Veremos que isto é conveniente quando introduzirmos o algorítmo de Tŭ̌-Zwart, na seção (2.4.1).

Para facilitar futuras referências, introduziremos a seguinte definição:

Definição 2.10 (Capacidades viáveis em relação a $f \in \mathbb{R}_{+}^{m}: C_{f}$ ) Para $f \in \mathbb{R}_{+}^{m}$ definimos o conjunto

$$
C_{f}=\left\{\begin{array}{l|l}
c \in \mathbb{R}_{+}^{m} & \begin{array}{l}
c_{I(f)}>f_{I(f)} \\
c_{e}=0 \text { se } f_{e}=0 \\
T(f, c) \leqq T_{M A X} .
\end{array}
\end{array}\right\}
$$


Note que o conjunto $C_{f}$ definido acima não corresponde exatamente a todas as capacidades viáveis em relação a $f$, mas somente àquelas "interessantes" no seguinte sentido: se $f \in \mathbb{R}_{+}^{m}$ e $c \in \mathbb{R}_{+}^{m}$ é tal que $c_{I(f)}>f_{I(f)}$ mas $c_{e}>0$ para algum $e \notin I(f)$, então a capacidade $c^{\prime}$ definida por

$$
c_{e}^{\prime}= \begin{cases}c_{e} & \text { se } e \in I(f) \\ 0 & \text { se } e \notin I(f)\end{cases}
$$

também satisfaz $c_{I(f)}^{\prime}>f_{I(f)}$, mas além disso satisfaz $D\left(c^{\prime}\right)<D(c)$. É claro que se uma determinada solução tem fluxo nulo em um arco, será economicamente melhor simplesmente não instalar o canal correspondente.

O problema $P D\left(T_{M A X}\right)$ pode ser reformulado como segue:

Definição 2.11 (Função $D^{*}\left(\cdot, T_{M A X}\right)$ ) Seja $T_{M A X}>0$. Definimos

$$
D^{*}\left(f, T_{M A X}\right)=\min \left\{\sum_{e \in I(f)} d_{e}\left(c_{e}\right) \mid c \in C_{f}\right\} .
$$

onde usamos min ao invés de inf pois, como já notado na seção anterior, o problema $P D\left(T_{M A X}\right)$ tem solução ótima para $T_{M A X}>0$.

Definição 2.12 (CFA)

$$
(C F A)\left\{\begin{array}{cc}
\min & D^{*}\left(f, T_{M A X}\right) \\
\text { s.a. } & f \in \mathcal{F}
\end{array}\right.
$$

Para simplificar a notação, escreveremos a partir de agora $D^{*}(f)=D^{*}\left(f, T_{M A X}\right)$, para $T_{M A X}>$ 0 da definição do problema.

Retomando nosso comentário em relação ao conjunto $C_{f}$, fica claro que a definição de $D^{*}(\cdot)$ não mudaria se permitíssemos que alguma capacidade $c_{e}>0$ com fluxo correspondente $f_{e}=0$, pelo simples fato de que tais capacidades podem sempre ser "melhoradas".

Esta reformulação tem a grande vantagem de retirar as capacidades do conjunto de pontos viáveis, fazendo com que o mesmo se reduza ao poliedro de fluxos multicomodidade. [Humes 88] provou (Fato IV.2.9) que se $D(\cdot)$ e $T(\cdot, \cdot)$ são definidas como em (2.8) e (2.9), então para cada $f \in \mathbb{R}_{+}^{m}$ existe e é único um $c^{*}(f)$ tal que $D^{*}(f)=D\left(c^{*}(f)\right)$, e que este $c^{*}(f)$ satisfaz $T\left(f, c^{*}(f)\right)=T_{M A X}$. Portanto, podemos enunciar a seguinte definição:

Definição 2.13 (Capacidade Ótima Associada a $f \in \mathbb{R}_{+}^{m}: c^{*}(\cdot)$ ) Para $f \in \mathbb{R}_{+}^{m}$ definimos

$$
c^{*}(f)=\operatorname{argmin}\left\{D(c) \mid c \in C_{f}\right\} .
$$

Esta função tem a propriedade de, para todo $f \in \mathbb{R}_{+}^{m}$,

$$
D^{*}(f)=D\left(c^{*}(f)\right) \text { e } T\left(f, c^{*}(f)\right)=T_{M A X}
$$


Para o caso de custos lineares podemos obter fórmulas fechadas para $c^{*}(\cdot)$ e $D^{*}(\cdot)$ :

Proposição 2.14 (Expressão de $c^{*}(\cdot)$ e $D^{*}(\cdot)$ para o caso de custos lineares) No caso de todas as funções $d_{e}(\cdot)$ serem lineares, isto é, $d_{e}\left(c_{e}\right)=l_{e} c_{e}$, então

$$
\begin{gathered}
c_{e}^{*}(f)=f_{e}+\frac{\sqrt{f_{e} / l_{e}} \sum_{k \in E} \sqrt{l_{k} f_{k}}}{\hat{q} T_{M A X}}, \forall e \in E, \\
D^{*}(f)=\sum_{e \in E} l_{e} f_{e}+\frac{\left(\sum_{e \in E} \sqrt{l_{e} f_{e}}\right)^{2}}{\hat{q} T_{M A X}}
\end{gathered}
$$

Prova : As condições de Kuhn-Tucker são necessárias e suficientes para o problema que define $D^{*}(f)$, ao restringirmos o problema ao subespaço $C_{f}^{\prime}=\left\{c_{I(f)} \in \mathbb{R}^{\# I(f)} \mid c \in C_{f}\right\}$, pois a função objetivo deste problema é linear e o conjunto $C_{f}^{\prime}$ tem interior (no subespaço) não-vazio, satisfazendo a condição de qualificação de Slater (em relação à restrição de retardo). Logo a capacidade ótima é dada pelo sistema

$$
(K T)\left\{\begin{array}{c}
l_{e}-\frac{u^{*} f_{e}}{\left(c_{e}^{*}-f_{e}\right)^{2}}=0, \forall e \in I(f) \\
\sum_{e \in I(f)} \frac{f_{e}}{c_{e}^{*}-f_{e}}=\hat{q} T_{M A X}
\end{array}\right.
$$

Da primeira equação descobrimos que $c_{e}^{*}=f_{e}+\sqrt{\frac{u^{*} f_{e}}{l_{e}}}, \forall e \in I(f)$ e substituindo na segunda equação vemos que $\sqrt{u^{*}}=\frac{\sum_{k \in E} \sqrt{l_{k} f_{k}}}{\hat{q} T_{M A X}}$, logo

$$
c_{e}^{*}=f_{e}+\frac{\sqrt{f_{e} / l_{e}} \sum_{k \in E} \sqrt{l_{k} f_{k}}}{\hat{q} T_{M A X}}, \forall e \in E,
$$

sendo que a mesma expressão define automaticamente $c_{e}=0$ quando $f_{e}=0$. Substituindo na expressão de $D^{*}(f)$ temos:

$$
D^{*}(f)=\sum_{e \in E} l_{e}\left(f_{e}+\frac{\sqrt{f_{e} / l_{e}} \sum_{k \in E} \sqrt{l_{k} f_{k}}}{\hat{q} T_{M A X}}\right)=\sum_{e \in E} l_{e} f_{e}+\frac{\left(\sum_{e \in A} \sqrt{l_{e} f_{e}}\right)^{2}}{\hat{q} T_{M A X}} .
$$

O seguinte resultado é de fácil verificação:

Proposição 2.15 (Monotonicidade de $D^{*}(\cdot)$ ) A função $D^{*}(\cdot)$ definida em (2.11) é estritamente crescente, isto é, sempre que $f^{1} \leq f^{2}$, teremos $D^{*}\left(f^{1}\right)<D^{*}\left(f^{2}\right)$.

Prova : Seja $\bar{c}=c^{*}\left(f^{2}\right)$ como em (2.13). Notamos que $\bar{c}_{I\left(f^{1}\right)}>f_{I\left(f^{1}\right)}^{2} \geqq f_{I\left(f^{1}\right)}^{1}$ e $T\left(f^{1}, \bar{c}\right)<T\left(f^{2}, \bar{c}\right) \leqq T_{M A X}$. Como $T(f, \cdot)$ é continua em $\bar{c}$, existe um $\varepsilon>0$ tal que

$$
c_{e}^{\prime}=\left\{\begin{array}{ll}
\bar{c}_{e}-\varepsilon & \text { se } e \in I\left(f^{1}\right) \\
0 & \text { se } e \notin I\left(f^{1}\right)
\end{array},\right.
$$


satisfaz $T\left(f^{1}, \bar{c}\right)<T\left(f^{1}, c^{\prime}\right) \leqq T_{M A X}$ e $c_{I(f)}^{\prime}>f_{I(f)}^{1}$, logo $c^{\prime} \in C_{f^{1}}$. Mas $c^{\prime} \leq \bar{c}$, logo $D\left(c^{\prime}\right)<D(\bar{c})$, e conseqüentemente

$$
D^{*}\left(f^{1}\right)=\min \left\{D(c) \mid c \in C_{f^{1}}\right\} \leqq D\left(c^{\prime}\right)<D(\bar{c})=D^{*}\left(f^{2}\right) .
$$

Este resultado permite que afirmemos

Corolário 2.16 ((CFA) sobre poliedro limitado) O problema definido em (2.12) é equivalente a

$$
(C F A)\left\{\begin{array}{lc}
\min & D^{*}(f) \\
\text { s.a. } & f \in[V(\mathcal{F})]
\end{array}\right.
$$

Prova : Basta notar que se $f=f^{\prime}+h \in \mathcal{F}$ onde $h \in C(\mathcal{F})$, então por $(2.15)$ $D^{*}(f) \leqq D^{*}\left(f^{\prime}\right)$. Logo

$$
\begin{array}{cl}
\min & D^{*}(f) \\
\text { s.a. } & f \in \mathcal{F}
\end{array}=\begin{array}{cc}
\min & D^{*}(f) \\
\text { s.a. } & f \in[V(\mathcal{F})]
\end{array} .
$$

O próximo resultado (de [Humes 88]) mostra que a função $D^{*}(\cdot)$ é côncava (estritamente côncava no caso de custos estritamente côncavos):

Proposição 2.17 (Concavidade de $D^{*}(\cdot)$ ) A função $D^{*}(\cdot)$ definida em (2.11) é côncava; além disso $D^{*}(\cdot)$ é estritamente côncava se os custos são estritamente côncavos.

Prova : Fazendo a mudança de variáveis $\rho_{e}=f_{e} / c_{e}$, temos

$$
D^{*}(f)=\inf \left\{\sum_{e \in I(f)} d_{e}\left(f_{e} / \rho_{e}\right) \mid \rho \in R(f)\right\},
$$

onde $R(f)=\left\{\rho \in \mathbb{R}^{m} \mid \sum_{e \in I(f)} \frac{\rho_{e}}{1-\rho_{e}} \leqq \hat{q} T_{M A X}\right.$ e $\left.\rho_{e} \in(0,1)\right\}$.

Considere então $f^{1}, f^{2} \in \mathbb{R}_{+}^{m}$ e $\lambda \in(0,1)$. Seja $f^{\lambda}=\lambda f^{1}+(1-\lambda) f^{2}$. Queremos mostrar que $D^{*}\left(f^{\lambda}\right)>\lambda D^{*}\left(f^{1}\right)+(1-\lambda) D^{*}\left(f^{2}\right)$.

Note inicialmente que, como $I\left(f^{1}\right) \cup I\left(f^{2}\right) \subset I\left(f^{\lambda}\right)$, temos que $R\left(f^{\lambda}\right) \subset R\left(f^{1}\right) \cap R\left(f^{2}\right)$. Lembrando que $D(\cdot)$ é côncava, teremos

$$
\begin{aligned}
D^{*}\left(f^{\lambda}\right)= & \inf \left\{\sum_{e \in I\left(f^{\lambda}\right)} d_{e}\left(f_{e}^{\lambda} / \rho_{e}\right) \mid \rho \in R\left(f^{\lambda}\right)\right\} \\
\geqq & \inf \left\{\sum_{e \in I\left(f^{\lambda}\right)} \lambda d_{e}\left(f_{e}^{1} / \rho_{e}\right)+(1-\lambda) d_{e}\left(f_{e}^{2} / \rho_{e}\right) \mid \rho \in R\left(f^{\lambda}\right)\right\} \\
\geqq & \lambda \inf \left\{\sum_{e \in I\left(f^{1}\right)} d_{e}\left(f_{e}^{1} / \rho_{e}\right) \mid \rho \in R\left(f^{\lambda}\right)\right\} \\
& +(1-\lambda) \inf \left\{\sum_{e \in I\left(f^{2}\right)} d_{e}\left(f_{e}^{2} / \rho_{e}\right) \mid \rho \in R\left(f^{\lambda}\right)\right\} \\
\geqq & \lambda D^{*}\left(f^{1}\right)+(1-\lambda) D^{*}\left(f^{2}\right),
\end{aligned}
$$

sendo que a primeira desigualdade será estrita se $D(\cdot)$ é estritamente côncava. Isto prova o enunciado. 
Se a função custo $D(\cdot)$ é linear, não podemos garantir a concavidade estrita da função $D^{*}(\cdot)$, como é erroneamente afirmado em [Humes 88]. Como contra-exemplo, tomemos a expressão fechada de $D^{*}(\cdot)$ dada em (2.14). Considere a diagonal

$$
\Delta=\left\{f \in \mathbb{R}_{+}^{m} \mid f=(z, z, \ldots, z) \text { com } z \in \mathbb{R}_{+}\right\},
$$

e note que na intersecção $\mathcal{F} \cap \Delta$ a expressão de $D^{*}(\cdot)$ se resume a

$$
D^{*}\left(\left[\begin{array}{c}
z \\
\vdots \\
z
\end{array}\right]\right)=z\left(\sum_{e \in E} l_{e}+\frac{\left(\sum_{e \in E} \sqrt{l_{e}}\right)^{2}}{\hat{q} T_{M A X}}\right),
$$

que é linear. Mas como provaremos em (3.40), a interseç̧ão $\mathcal{F} \cap \Delta$ contém uma semi-reta, e portanto $D^{*}(\cdot)$ não é estritamente côncava em $\mathcal{F}$, não o sendo também no $\mathbb{R}_{+}^{m}$.

Porém o argumento apresentado por [Humes 88] pode ser parcialmente aproveitado para estabelecer um fato que nos será bastante importante:

Proposição 2.18 (Quasiconcavidade estrita da $D^{*}(\cdot)$ ) $D^{*}(\cdot)$ é estritamente quasicôncava.

Prova : No caso de custos estritamente côncavos temos por $(2.17)$ que $D^{*}(\cdot)$ é estritamente côncava, e por (1.2) $D^{*}(\cdot)$ é estritamente quasicôncava. Falta apenas provar o enunciado para o caso de custos lineares.

Pela proposição (2.14) sabemos que $D^{*}(\cdot)$ é dada por

$$
D^{*}(f)=\sum_{e \in E} l_{e} f_{e}+\frac{\left(\sum_{e \in E} \sqrt{l_{e} f_{e}}\right)^{2}}{\hat{q} T_{M A X}} .
$$

Sejam $f^{1}, f^{2} \in \mathbb{R}_{+}^{m}$, com $f^{1} \neq f^{2}$. Sabemos que para todo $\lambda \in(0,1)$, $I\left(\lambda f^{1}+(1-\lambda) f^{2}\right)=I\left(f^{1}\right) \cup I\left(f^{2}\right)$. Seja $p=\# I\left(f^{1}\right) \cup I\left(f^{2}\right)$. Mostraremos que $D^{*}(\cdot)$ é estritamente quasicôncava no subespaço

$$
F^{\prime}=\left\{f \in \mathbb{R}_{+}^{m} \mid f=\lambda f^{1}+(1-\lambda) f^{2} \text { para algum } \lambda \in(0,1)\right\} .
$$

Para tanto, utilizaremos o hessiano da restrição de $D^{*}(\cdot)$ a $F^{\prime}$. Este é uma matriz múltipla positiva da matriz $H=\left(h_{i j}\right)$ dada por

$$
h_{i j}= \begin{cases}-\sum_{k \neq i} \sqrt{\frac{l_{k} f_{k}}{\left(l_{i} f_{i}\right)^{3}}}, & \text { para } i=j, \\ \frac{1}{\sqrt{l_{i} f_{i} l_{j} f_{j}}}, & \text { para } i \neq j .\end{cases}
$$

Seja $f \in F^{\prime}$ qualquer tal que $f_{I(f)} \in F^{\prime}$ e seja $d=f^{2}-f^{1} \neq 0$. Então (supondo $I(f)=\{1,2, \ldots, p\}$ para facilitar a notação)

$$
\begin{aligned}
d^{t} H d & =\sum_{i=1}^{p} \sum_{j \neq i}-d_{i}^{2} \sqrt{\frac{l_{j} f_{j}}{\left(l_{i} f_{i}\right)^{3}}}+\sum_{i=1}^{p} \sum_{j \neq i} d_{i} d_{j} \sqrt{\frac{1}{l_{i} f_{i} l_{j} f_{j}}} \\
& =\sum_{i=1}^{p} \sum_{j>i}\left(-d_{i}^{2} \sqrt{\frac{l_{i} f_{i}}{\left(l_{j} f_{j}\right)^{3}}}+2 d_{i} d_{j} \sqrt{\frac{1}{l_{i} f_{i} l_{j} f_{j}}}-d_{j}^{2} \sqrt{\frac{l_{j} f_{j}}{\left(l_{i} f_{i}\right)^{3}}}\right) \\
& =\sum_{i=1}^{p} \sum_{j>i}-\left(d_{i}-\frac{l_{i} f_{i}}{l_{j} f_{j}} d_{j}\right)^{2} \sqrt{\frac{l_{i} f_{i}}{\left(l_{j} f_{j}\right)^{3}}} \\
& \leqq 0
\end{aligned}
$$


Temos dois casos: $d^{t} H d=0$ e $d^{t} H d<0 . \quad$ Note que $d^{t} H d=0 \Longleftrightarrow\left(d_{i}-\frac{l_{i} f_{i}}{l_{j} f_{j}} d_{j}\right)=0, \forall i=1, \ldots, p, \forall j>i$, e neste caso ou $d \leq 0$ ou $d \geq 0$, isto é, ou $f^{2} \leq f^{1}$ ou $f^{2} \geq f^{1}$; pela monotonicidade estrita (2.15) e lembrando que $D^{*}(\cdot)$ é côncava $(2.17)$, temos que $\forall \lambda \in(0,1)$,

$$
\begin{aligned}
D^{*}\left(\lambda f^{1}+(1-\lambda) f^{2}\right) \geqq & \lambda D^{*}\left(f^{1}\right)+(1-\lambda) D^{*}\left(f^{2}\right) \\
> & \lambda \min \left\{D^{*}\left(f^{1}\right), D^{*}\left(f^{2}\right)\right\} \\
& +(1-\lambda) \min \left\{D^{*}\left(f^{1}\right), D^{*}\left(f^{2}\right)\right\} \\
= & \min \left\{D^{*}\left(f^{1}\right), D^{*}\left(f^{2}\right)\right\} .
\end{aligned}
$$

Se $d^{t} H d<0$, temos [Mangasarian 69] que $D^{*}(\cdot)$ é estritamente côncava no ponto $f$ em relação ao segmento aberto $F^{\prime}$. Logo existe um $\varepsilon>0$ tal que $(f-\varepsilon d, f+\varepsilon d) \in F^{\prime} \times F^{\prime}$ e

$$
D^{*}(f)>\frac{1}{2} D^{*}(f-\varepsilon d)+\frac{1}{2} D^{*}(f+\varepsilon d) .
$$

Além disso, como a função $D^{*}(\cdot)$ é côncava, esta será também quasicôncava (1.2). Isto significa que

$$
D^{*}(f-\varepsilon d) \geqq \min \left\{D^{*}\left(f^{1}\right), D^{*}\left(f^{2}\right)\right\}
$$

$\mathrm{e}$

$$
D^{*}(f+\varepsilon d) \geqq \min \left\{D^{*}\left(f^{1}\right), D^{*}\left(f^{2}\right)\right\}
$$

que junto com a desigualdade anterior fornece

$$
D^{*}(f)>\min \left\{D^{*}\left(f^{1}\right), D^{*}\left(f^{2}\right)\right\}
$$

completando a prova.

Outro belo resultado (de [Humes 88$]$ ) sobre a função $D^{*}(\cdot)$ segue.

Proposição 2.19 (Supergradiente de $D^{*}(\cdot)$ ) Dado $\bar{f} \in \mathbb{R}_{+}^{m}, \bar{f}>0$, seja $\bar{c}=c^{*}(\bar{f})$; o vetor $W$ definido por

$$
W_{e}=\nabla_{e} D(\bar{c})+\frac{\sqrt{\nabla_{e} D(\bar{c})} \sum_{k \in E} \sqrt{\nabla_{k} D(\bar{c}) \bar{f}_{k}}}{\hat{q} T_{M A X} \sqrt{\bar{f}_{e}}}, \forall e \in E
$$

é um supergradiente da função $D^{*}(\cdot)$ no ponto $\bar{f}$.

Prova : Defina a função $D_{L}(f)$ por

$$
D_{L}(f)=\inf _{c \in C_{f}}\langle\nabla D(\bar{c}), c-\bar{c}+D(\bar{c}) .
$$

Note inicialmente que $D_{L}(\bar{f})=D^{*}\left(\bar{f}, T_{M A X}\right)$, pois 


$$
\begin{aligned}
D_{L}(\bar{f}) & \left.=\inf _{c \in C_{\bar{f}}} \nabla \nabla(\bar{c}), c-\bar{c}\right)+D(\bar{c}) \\
& \geqq \inf _{c \in C_{\bar{f}}} D(c) \\
& =D^{*}(\bar{f}) \\
& =D(\bar{c}) \\
& \geqq \inf _{c \in C_{\bar{f}}}\left\langle\nabla D(\bar{c}), c-\bar{c}+D(\bar{c})=D_{L}(\bar{f}) ;\right.
\end{aligned}
$$

a primeira desigualdade é conseqüência da concavidade da função $D(\cdot)$, e a segunda vem do fato de $\bar{c} \in C_{\bar{f}}$.

Além disso, para todo $f \in \mathbb{R}_{+}^{m}$, temos que $D_{L}(f) \geqq D^{*}(f)$, pois

$$
\begin{aligned}
D_{L}(f) & =\inf _{c \in C_{f}}\langle\nabla D(\bar{c}), c-\bar{c})+D(\bar{c}) \\
& \geqq \inf _{c \in C_{f}} D(c) \\
& =D^{*}(f)
\end{aligned}
$$

Agora, pelas condições de Kuhn-Tucker podemos calcular $D_{L}(f)$, exatamente pelas mesmas razões e da mesma maneira que foi feito na prova da proposição (2.14). Temos então que resolver o sistema (nas variáveis $c_{e}^{*}$ e multiplicador $u^{*}$ ):

$$
(K T)\left\{\begin{aligned}
\nabla_{e} D(\bar{c})-\frac{u^{*} f_{e}}{\left(c_{e}^{*}-f_{e}\right)^{2}} & =0, \forall e \in I(f) \\
\sum_{e \in I(f) \frac{f_{e}}{c_{e}^{*}-f_{e}}} & =\hat{q} T_{M A X}
\end{aligned}\right.
$$

cuja solução é

$$
\left\{\begin{aligned}
c_{e}^{*} & =f_{e}+\frac{\sqrt{f_{e} / \nabla_{e} D(\bar{c})}}{\hat{q} T_{M A X}} \sum_{h \in I(f)} \sqrt{\nabla_{h} D(\bar{c}) f_{h}}, \forall e \in I(f) \\
\sqrt{u^{*}} & =\frac{\sum_{h \in I(f)} \sqrt{\nabla_{h} D(\bar{c}) f_{h}}}{\hat{q} T_{M A X}} .
\end{aligned}\right.
$$

Temos então uma expressão fechada para $D_{L}(f)$, fazendo $c_{e}^{*}=0$ para $f_{e}=0$ :

$$
\begin{aligned}
D_{L}(f) & =D(\bar{c})+\nabla D(\bar{c}), c^{*}-\bar{c} \\
& =D(\bar{c})-\sum_{e \in E} \nabla_{e} D(\bar{c}) \bar{c}_{e}+\sum_{e \in E} \nabla_{e} D(\bar{c}) c_{e}^{*} \\
& =D(\bar{c})-\sum_{e \in E} \nabla_{e} D(\bar{c}) \bar{c}_{e}+\sum_{e \in E}\left(\nabla_{e} D(\bar{c}) f_{e}+\frac{\left(\sum_{h \in E} \sqrt{\nabla_{h} D(\bar{c}) f_{h}}\right)^{2}}{\hat{q} T_{M A X}}\right)
\end{aligned}
$$

Com esta expressão somos capazes de calcular o gradiente $W$ da função $D_{L}(\cdot)$ no ponto $\bar{f}$ :

$$
W_{e}=\nabla_{e} D(\bar{c})+\frac{\sqrt{\nabla_{e} D(\bar{c})} \sum_{h \in E} \sqrt{\nabla_{h} D(\bar{c}) \bar{f}_{h}}}{\hat{q} T_{M A X} \sqrt{\bar{f}_{e}}}, \forall e \in E
$$

O resultado de que o gradiente de $D_{L}(\cdot)$ em $\bar{f}$ é um supergradiente de $D^{*}(\cdot)$ em $\bar{f}$ segue dos fatos de que $D^{*}(\cdot)$ é côncava $(2.17), D_{L}(\cdot) \geqq D^{*}(\cdot), D_{L}(\bar{f})=D^{*}(\bar{f})$, e $D_{L}(\cdot)$ é diferenciável em $\bar{f}$. 
Embora tenhamos restringido o resultado acima para fluxos $\bar{f}>0$, é claro que o resultado vale para qualquer $\bar{f} \in \mathcal{F}$ dentro do subespaço $F=\{f \in \mathcal{F} \mid I(f) \equiv I(\bar{f})\}$, onde as únicas coordenadas do supergradiente que estarão definidas são aquelas associadas a $\bar{f}_{e}>0$.

É importante notar o comportamento do supergradiente quando algum $f_{e}$ tende a 0 ; pela expressão dada em (2.19) vemos que $W_{e} \longrightarrow \infty$. Este comportamento, que batizamos de efeito "calha", por causa do exemplo seguinte, voltará a ser mencionado na seção (2.5).

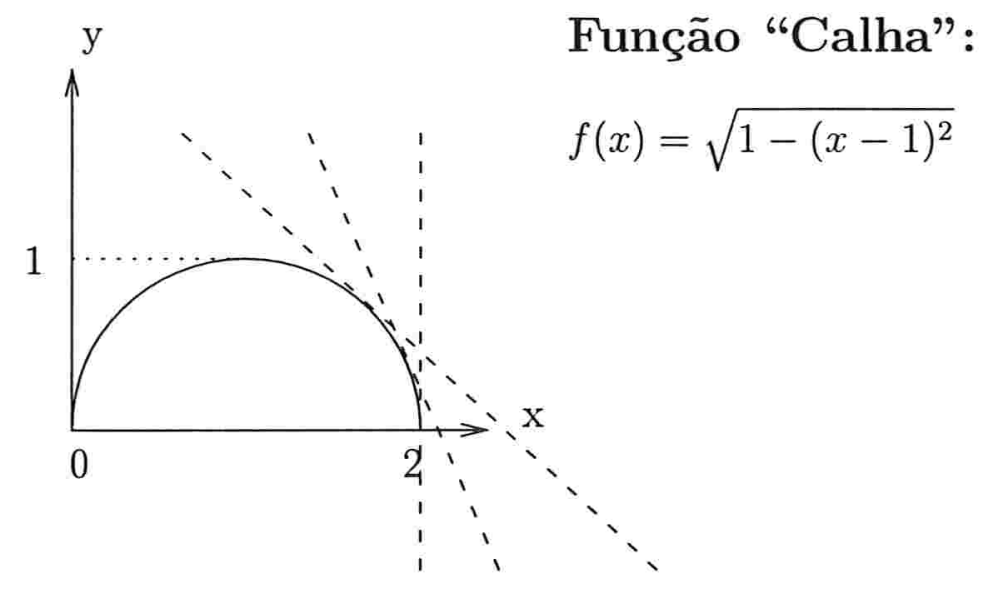

\subsubsection{Técnica de linearizações externas para o cálculo de $D^{*}(\cdot)$}

Em [Humes 88] e [Humes 92] podemos encontrar a descrição de um método para calcular $D^{*}(f)$ que utiliza linearizações externas, como descrevemos a seguir.

Note que, dado $f \in \mathbb{R}_{+}^{m}$, computar $D^{*}(f)$ equivale a resolver o problema

$$
(C A) \begin{cases}\text { minimizar } & D(c) \\ \text { s.a. } & c \in C_{f}\end{cases}
$$

conhecido como designação de capacidades (Capacity Assignment).

Como vimos na proposição (2.14) a solução para este problema admite uma expressão fechada no caso de custos lineares. Esta expressão é

$$
c_{e}^{*}(f)=f_{e}+\frac{\sqrt{f_{e} / l_{e}} \sum_{k \in E} \sqrt{l_{k} f_{k}}}{\hat{q} T_{M A X}}, \forall e \in E ;
$$

Este fato pode ser aproveitado como descrito em [Humes 88] e [Humes 91] para resolver o (CA) com $D(c)=\sum_{e \in E} l_{e} c_{e}^{\alpha}$ e $\alpha \in(0,1]$. O método baseia-se em linearizações da função objetivo, da seguinte forma: 
Algorítmo 2 (Cálculo de $D^{*}(f)$ )

1. Encontre um $c^{0}$ viável para o (CA), por exemplo, tomando

$$
c_{e}^{0}=f_{e}\left(\frac{m}{\hat{q} T_{M A X}}+1\right) .
$$

Faça $k=0$.

2. Resolva o problema

$$
(C A)_{k} \begin{cases}\text { minimizar } & \sum_{e \in E}\left[l_{e}\left(c_{e}^{k}\right)^{\alpha}+l_{e}\left(c_{e}^{k}\right)^{\alpha-1}\left(c_{e}-c_{e}^{k}\right)\right] \\ \text { s.a. } & c \in C_{f}\end{cases}
$$

3. Chame a solução encontrada de $c^{k+1}$, faça $k \longleftarrow k+1$ e volte ao passo 2.

Como termos constantes na expressão a ser minimizada no passo 2 podem ser eliminados, queremos na realidade em $(C A)_{k}$ minimizar a expressão $\sum_{e \in E} l_{e}\left(c_{e}^{k}\right)^{\alpha-1} c_{e}$, e aplicando a expressão que já temos para o caso linear temos que a solução ótima do $(C A)_{k}$ é dada por

$$
c_{e}^{k+1}=f_{e}+\frac{1}{\hat{q} T_{M A X}} \sqrt{\frac{f_{e}}{l_{e}\left(c_{e}^{k}\right)^{\alpha-1}}} \sum_{h \in E} \sqrt{f_{h} l_{h}\left(c_{h}^{k}\right)^{\alpha-1}} .
$$

A sequência $\left\{c^{k}\right\}_{k \in \mathbb{N}}$ gerada é compacta (está em um compacto) e converge para o ótimo do (CA) [Humes 91]. Destacamos neste método a extrema eficiência e simplicidade de implementação, quando comparado com o método de Newton-Raphson para a solução do sistema não-linear dado pelas equações de Kuhn-Tucker correspondentes ao (CA). Destacamos também o fato deste método trabalhar apenas com 1 linearização a cada passo, sendo que geralmente os métodos de linearização trabalham com $m+1$ linearizações anteriores a cada passo.

\subsection{Os Algoritmos de Tǔ̆-Zwart e Falk-Hoffman}

Considere o problema

Definição 2.20 (Problema Geral de Programação Côncava) Encontre

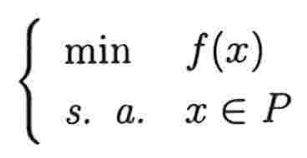

onde $f(\cdot)$ é uma função côncava e $P$ é um poliedro.

Lembramos que se o problema acima possui uma solução ótima, então existe um vértice que é minimizador da função $f$ (Proposição (1.21)). O que torna este problema difícil é a presença 
de mínimos locais que não são globais: podemos construir exemplos em que todos os vértices de $P$ são mínimos locais, mas apenas um deles é o mínimo global. Problemas com esta propriedade originaram a área de estudo chamada de Otimização Global, sendo Programação Côncava uma sub-área desta. Estudaremos neste capítulo dois métodos de resolução do problema (2.20), um devido a Tŭ e Zwart, outro devido a Falk e Hoffman.

\subsubsection{Tŭ-Zwart}

A primeira proposta para resolver o problema geral de Programação Côncava foi feita por [Tuı̆ 64]. Algum tempo depois [Zwart 74] mostrou que o algoritmo de Tŭ poderia não convergir em alguns exemplos, e propôs um algoritmo muito parecido, embora desenvolvido independentemente de Tǔ̆, cuja convergência finita foi provada.

Estes dois algoritmos têm como ponto central um procedimento de corte de certas regiões do poliedro nas quais o valor da função objetivo é certamente pior do que o valor atualmente conhecido. São os chamados cortes de concavidade. Chamamos a atenção no sentido de evitar a confusão entre estes cortes dos poliedros e os cortes em grafos, definidos em (1.37). Para introduzi-los, precisamos da seguinte definição:

Definição 2.21 (Vértices mínimos locais em relação aos seus vizinhos) Seja $P \subset \mathbb{R}^{n}$ um poliedro e $\bar{v} \in V(P)$. Seja $f: X \longrightarrow \mathbb{R}$ uma função quasicôncava, onde $P \subset X$. Dizemos que $v$ é um mínimo local em relação aos seus vizinhos (em relação à função objetivo $f(\cdot))$ se $f(\bar{v}) \leqq \min \{f(v) \mid v$ é vizinho de $\bar{v}\}$, e se $f(\bar{v})=\min \{f(x) \mid x \in B\}$, para toda aresta ilimitada $B$ saindo de $\bar{v}$.

Note que a definição acima é equivalente a dizer que $\bar{v}$ minimiza $f(\cdot)$ no conjunto dado pela união das arestas saindo de $\bar{v}$.

Neste trabalho o conceito de mínimo local no seu sentido usual não será mais utilizado, e freqüentemente diremos apenas que $\bar{v} \in V(P)$ é um mínimo local, no sentido acima definido.

Definição 2.22 (Extensão côncava) Seja $f: X \longrightarrow \mathbb{R}^{q}$ uma função côncava, e $Y$ tal que $X \subset Y$. Dizemos que $g: Y \longrightarrow \mathbb{R}^{q}$ é uma extensão côncava de $f$ em $Y$ se $g$ é côncava, $g(x)=f(x), \forall x \in X$, e $g$ é maximal em relação a estas propriedades.

É importante notar que, embora [Tư̆ 64] afirme que toda função côncava possui uma extensão côncava, existem funções cuja "extensão côncava" tem valores em $\overline{\mathbb{R}}$, como no caso da função "calha" do final da seção anterior, para $Y=\mathbb{R}$.

Assumimos então que a função $f$ está definida em todo o $\mathbb{R}^{n}$ (do contrário tomamos uma extensão côncava de $f$ ).

Definimos então os cortes de concavidade: 
Definição 2.23 (Corte de Concavidade - Corte de Tư̌) Seja $P \subset \mathbb{R}^{n}$ um poliedro nãodegenerado de dimensão $n$ e $\bar{v} \in V(P)$ um mínimo local. Sejam $d^{1}, d^{2}, \ldots, d^{n}$ as direções de arestas saindo de $\bar{v}$. Para cada direção $d^{k}$ tome

$$
\begin{aligned}
\theta_{k} & =\arg \max \left\{\theta \mid \theta \geqq 0, \text { e } f\left(\bar{v}+\theta d^{k}\right) \geqq f(\bar{v})\right\} \\
x^{k} & =\bar{v}+\theta_{k} d^{k}
\end{aligned}
$$

(se $d^{k}$ for ilimitada, escolha $x^{k}=\bar{v}+\theta d^{k} \operatorname{com} \theta>0$ um ponto arbitrariamente distante de $\bar{v})$. Os $n$ pontos acima $\left\{x^{1}, x^{2}, \ldots, x^{n}\right\}$ definem um único semi-espaço $\{\langle a, x\rangle \leqq b\}$ que contém todos os vértices vizinhos de $\bar{v}$, satisfazendo $\left\langle a, x^{2}\right\rangle=b$ e $\langle a, \vec{v}\rangle<b$. Como $f(x) \geqq f(\bar{v})$, $\forall x \in P \cap\{\langle a, x\rangle \leqq b\}$, segue que

$$
\min \{f(x) \mid x \in P\}=\min \{f(\bar{v}), \min \{f(x) \mid x \in\{\langle a, x\rangle \geqq b\} \cap P\}\}
$$

Chamamos à restrição $\{\langle a, x\rangle \geqq b\}$ o corte de concavidade ou corte de Tŭ gerado por $\bar{v}$.

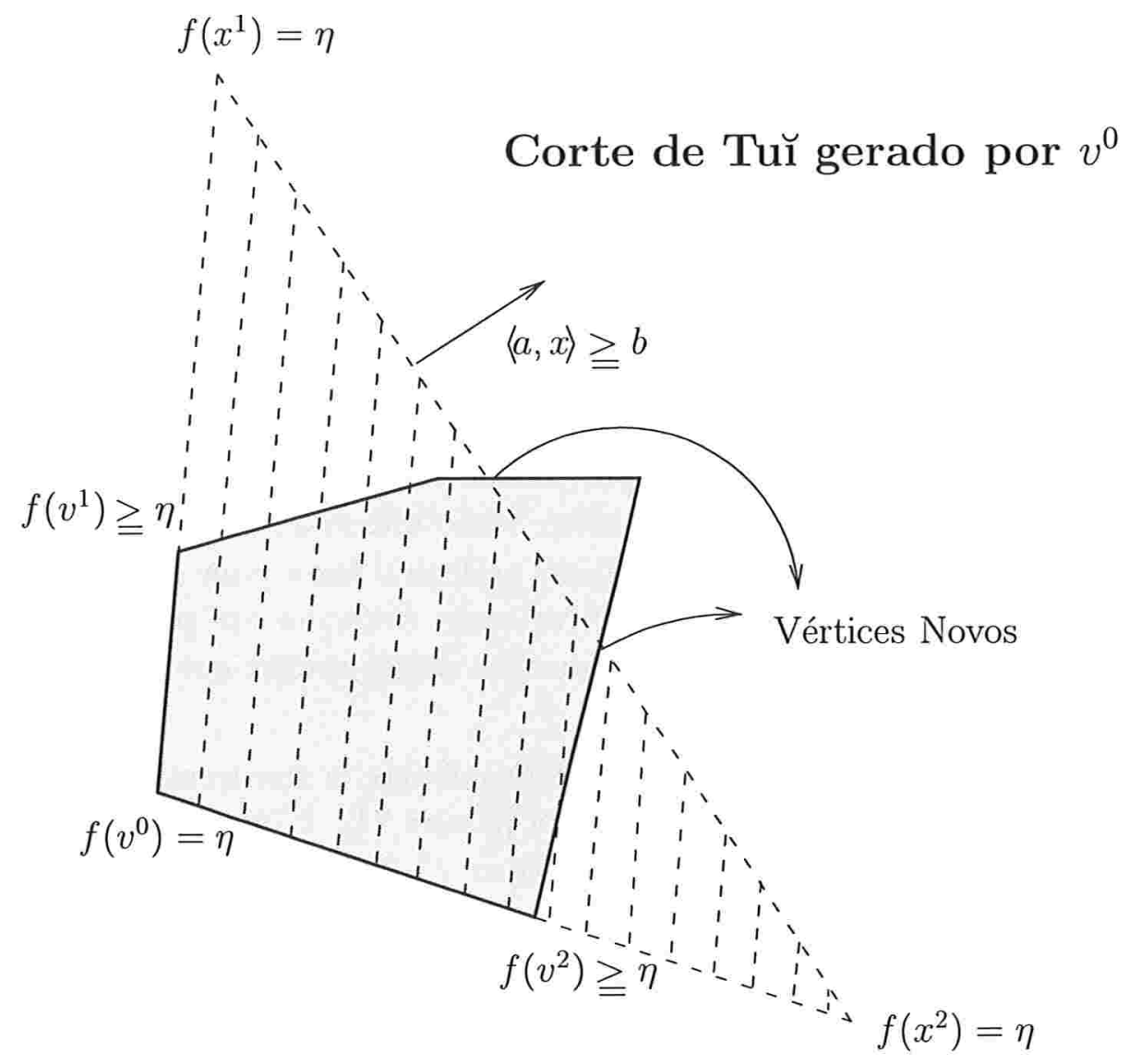

Os algoritmos de Tŭ e Zwart, em suas formulações originais, utilizam os cortes de concavidade para definir uma família de subproblemas de tal forma que a solução do problema original é a 
melhor dentre as soluções dos subproblemas. É claro que cada um destes subproblemas, introduzindo novos cortes de concavidade, criarão sub-subproblemas; Tŭ não prova qualquer limitação no número de subproblemas introduzidos, e Zwart prova apenas que estes são em número finito, não fornecendo qualquer limitação em função do tamanho do problema. Uma hipótese fundamental para a validade desta prova é de que o poliedro seja limitado.

Optamos por utilizar uma variante destes algoritmos, que é enunciada pelos dois autores (em [Tuй 64] e [Zwart 74]):

Algorítmo 3 (Tǔ̆-Zwart) Seja $P=\{x \mid A x \leqq b\} \subset \mathbb{R}^{n}$ um poliedro limitado nãodegenerado de dimensão $n$, e $f: \mathbb{R}^{n} \longrightarrow \mathbb{R}$ uma função côncava. Seja $\varepsilon>0$.

1. Faça Valor Ótimo $\longleftarrow \infty$.

2. Encontre um mínimo local $\bar{v} \in V(P) ; \quad S e \quad f(\bar{v}) \quad<$ Valor Ótimo, faça Valor Ótimo $\longleftarrow f(\bar{v})$, e Ótimo $\longleftarrow \bar{v}$.

3. Determine o corte de concavidade $\{\langle a, x\rangle \geqq b\}$ a partir de $\bar{v} ;$ Resolva o problema $\theta=\max \langle a, x\rangle$ no poliedro $P$.

4. $S e \theta \leqq b+\varepsilon$ pare!

5. Se $\theta>b+\varepsilon$, Faça $P \longleftarrow P \cap\{\langle a, x\rangle \geqq b+\varepsilon\}$, e volte para o passo 2.

Note que a hipótese de não-degenerescência torna o cálculo do corte de Tuŭ bem simples, ou seja, determinar qual o hiperplano que passa pelos $n$ pontos determinados na direção das arestas. O cálculo destes pontos é um problema de otimização uni-dimensional, para o qual vários métodos numéricos podem ser aplicados (ver por exemplo [Luenberger 73]). Deve-se notar que a prova de [Zwart 74] supõe que as atualizações de $P$ no passo 5 não introduzem degenerescência.

Notamos também que no passo 3 do algoritmo podemos fazer com que o corte de Tul̆ seja melhorado, fazendo com que as $n$ buscas uni-direcionais avancem até pontos $x^{k}$ com valor da função objetivo maior ou igual a Valor Ótimo, ou seja, exigir apenas que $f\left(x^{k}\right) \geqq$ Valor ótimo ao invés de $f\left(x^{k}\right) \geqq f(\bar{v})$.

Esta variante tem a vantagem de não gerar subproblemas, e sua terminação em um número finito de cortes (para $\varepsilon>0$ ) também foi indicada por [Zwart 74]. É claro que para $\varepsilon>0$ genérico, o passo 5 poderá eventualmente cortar a solução ótima $f^{*}$ (se acontecer de $b<\left\langle c, f^{*}\right\rangle\langle b+\varepsilon$ ); nisto o algorítmo depende de uma boa escolha de $\varepsilon$, que deverá ser feita caso-a-caso.

\subsubsection{Falk-Hoffman}

Uma abordagem diferente para a solução do problema (2.20) foi proposta por [Falk \& Hoffman 76]. Esta utiliza o conceito de envelope convexo de uma função: 
Definição 2.24 (Envelope convexo) Seja $f: X \longrightarrow \mathbb{R}^{n}$ uma função qualquer. O envelope convexo de $f$ em $T \subset X$ é uma função convexa $g:[T] \longrightarrow \mathbb{R}^{n}$ tal que $g(x) \leqq f(x), \forall x \in T$ e que é maximal no seguinte sentido: se $h$ é uma função convexa qualquer definida em $[T]$ tal que $h(x) \leqq f(x), \forall x \in T$, então $h(x) \leqq g(x), \forall x \in[T]$.

Os resultados a seguir podem ser encontrados em [Falk \& Hoffman 76]:

Proposição 2.25 (Condição necessária de otimalidade para programação côncava) Se $g$ é o envelope convexo de $f$ no conjunto $P$ de pontos viáveis de (2.20), então um minimizador global para este problema é tambem um minimizador de $g$ sobre $P$.

Proposição 2.26 (Envelope convexo de $f$ sobre $P$ ) Seja $P$ um poliedro limitado tal que $V(P)=\left\{v^{0}, v^{1}, \ldots, v^{k}\right\}$. O envelope convexo de uma função côncava $f$ sobre $P$ pode ser expresso como

$$
\begin{aligned}
g(x)=\min & \sum_{i=0}^{k} f\left(v^{i}\right) \alpha_{i} \\
\text { s. } a . & \sum_{i=0}^{k} v^{i} \alpha_{i}=x \\
& \sum_{i=0}^{k} \alpha_{i}=1 \\
& \alpha \geqq 0 .
\end{aligned}
$$

O algorítmo de Falk-Hoffman trabalha com uma seqüência $\left\{P^{k}\right\}_{k=0}^{l}$ de aproximações do poliedro original, sendo que cada $P^{k}$ é descrito por um subconjunto das facetas do poliedro original.

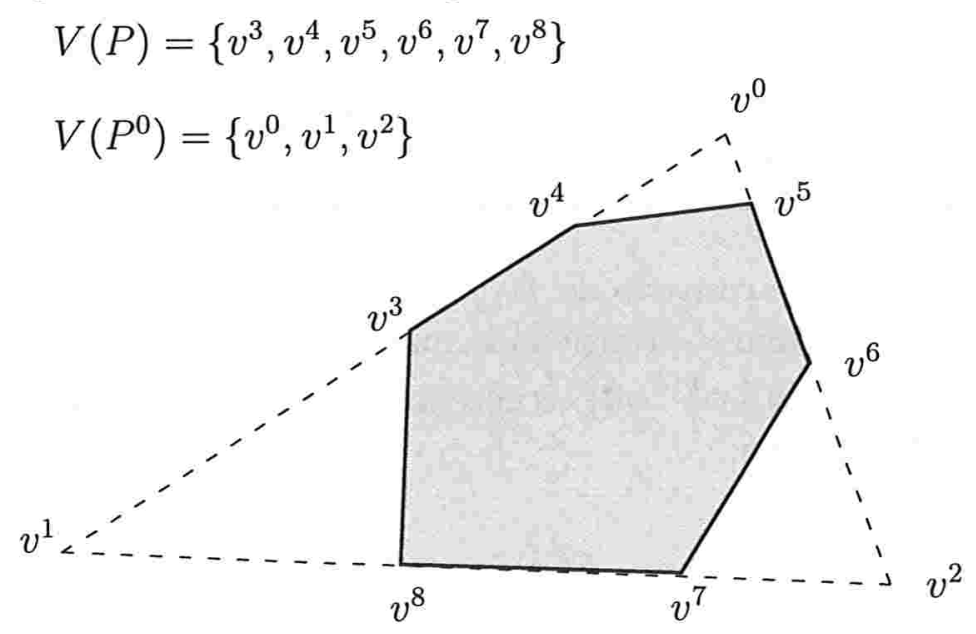

\section{Aproximações externas do poliedro utilizadas por Falk-Hoffman}


Algorítmo 4 (Falk-Hoffman) Seja $P=\{x \mid A x \leqq b\} \subset \mathbb{R}^{n}$ um poliedro limitado nãodegenerado, e $f: \mathbb{R}^{n} \longrightarrow \mathbb{R}$ uma função côncava.

1. Encontre um poliedro $P^{0}$ limitado e formado apenas por restrições do poliedro original, tal que $P \subset P^{0}$, e identifique todos os seus vértices. Seja $V^{0}=V\left(P^{0}\right)$.

2. Resolva o problema

$$
\left(Q^{k}\right) \begin{cases}\min & \sum_{v^{j} \in V^{k}} \alpha_{j} f\left(v^{j}\right) \\ s . a . & \sum_{v^{j} \in V^{k}} A v^{j} \alpha_{j} \leqq b \\ & \sum_{v^{j} \in V^{k}} \alpha_{j}=1 \\ & \alpha \geqq 0 .\end{cases}
$$

Sendo $\alpha^{k}$ sua solução, faça $x^{k}=\sum_{v^{j} \in V^{k}} \alpha_{j}^{k} v^{j}$ e seja $A^{k}=\left\{j \mid \alpha_{j}^{k}>0\right\}$ o conjunto de indices dos vértices realmente utilizados na representação de $x^{k}$.

3. Se $v^{j} \in P, \forall j \in A^{k}$, pare! $A$ solução ótima é $v^{*}=\operatorname{argmin}\left\{f\left(v^{j}\right) \mid v^{j} \in V^{k}\right\}$.

4. Caso contrário, existe algum $v^{j} \in V^{k}$ tal que $\alpha_{j}^{k}>0$ e $v^{j} \notin P$. Escolha alguma restrição do poliedro original violada por $v^{j}$ e faça $P^{k+1}$ ser o poliedro obtido de $P^{k}$ pela inclusão desta restrição. Calcule o conjunto $V^{k+1}$ de vértices do novo poliedro, e vá para o passo 2.

O critério de parada no passo 2 do algorítmo é justificado pela seguinte proposição:

Proposição 2.27 (Critério de parada de Falk-Hoffman) Seja $x^{k}=\sum_{v^{j} \in V^{k}} \alpha_{j}^{k} v^{j}$ a solução do problema $\left(Q^{k}\right)$ no passo 1 , e suponha que $v^{j} \in P$, para todos os $\alpha_{j}^{k}>0$. Então

$$
v^{*}=\arg \min \left\{f\left(v^{j}\right) \mid \alpha_{j}^{k}>0\right\}
$$

é um mínimo global para o problema (2.20).

Prova : Seja $F^{k}(\cdot)$ o envelope convexo de $f(\cdot)$ tomado em $P^{k} \supset P$, como descrito em (2.26). O envelope convexo tem a propriedade que $F^{k}(x) \leqq f(x)$, para todo $x \in P^{k}$. Utilizando o conjunto $A^{k}=\left\{j \mid \alpha_{j}^{k}>0\right\}$, como no algorítmo, e lembrando que $x^{k}$ é a solução ótima de $Q^{k}$, temos que:

$$
\begin{aligned}
F^{k}\left(x^{k}\right) & =F^{k}\left(\sum_{j \in A^{k}} \alpha_{J}^{k} v^{j}\right) \\
& =\sum_{j \in A^{k}} \alpha_{j}^{k} f\left(v^{j}\right) \\
& \geqq \min \left\{f\left(v^{j}\right) \mid j \in A^{k}\right\} \\
\geqq & \min \left\{F\left(v^{j}\right) \mid j \in A^{k}\right\} \\
\geqq & F^{k}\left(x^{k}\right) .
\end{aligned}
$$


E conseqüentemente $F^{k}\left(x^{k}\right)=\min \left\{f\left(v^{j} \mid j \in A^{k}\right\}=f\left(v^{*}\right)\right.$. Como $v^{*} \in P$ e $f\left(v^{*}\right)=F^{k}\left(x^{k}\right) \leqq F^{k}(x) \leqq f(x)$, para todo $x \in P^{k} \supset P$, temos que $v^{*}$ é solução ótima para o problema.

O procedimento descrito em (4) é finito, pois a cada passo é introduzida uma nova restrição do problema original. O número de linhas dos subproblemas e do problema original diferem de uma restrição, e o número de colunas nos subproblemas é limitado pelo número de vértices do problema original.

Embora nosso objetivo principal nesta dissertação seja abordar a solução do (CFA) pelo método de Tŭ-Zwart, voltaremos a comentar a possibilidade de aplicação de Falk-Hoffman para o (CFA) na seção (3.3.2).

\subsection{Problemas de Aplicabilidade de Tǔ̆-Zwart ao (CFA)}

Como vimos na seção anterior, uma das primeiras exigências do método de Tŭ-Zwart sobre o conjunto de pontos viáveis é que ele seja um poliedro limitado, não-degenerado da forma $A x \leqq b$, de dimensão $n$. Dos poliedros que vimos na seção $(2.2 .2)$, somente $\vec{F}^{r s}$ possui uma estrutura explícita da forma $A x=b$, tendo conseqüentemente interior vazio. Os outros poliedros são caracterizados por projeções e somas dos poliedros $\vec{F}^{r s}$.

A não-degenerescência e o interior não-vazio são hipóteses que garantem a facilidade de determinar um plano de corte para o poliedro. Poderíamos criar estratégias alternativas para a determinação do plano de corte sem estas hipóteses, desde que fôssemos capazes de determinar todos os vizinhos de um dado vértice no poliedro (ver seção (4.2)).

Um exemplo de poliedro com interior vazio para o qual não é dificil calcular o plano de corte de Tǔ é o poliedro canônico (1.9) não-degenerado. Seja $P=\left\{x \in \mathbb{R}^{n} \mid A x=b, x \geqq 0\right\}$ e $v$ uma solução básica viável de $P$, associada à base $B$; para simplificar a notação, assuma $B=\{1, \ldots, m\}$. Então temos exatamente $n-m$ direções de arestas saindo de $v$ (dadas em (1.34)):

$$
d^{k}=\left[\begin{array}{c}
-A_{B}^{-1} A^{k} \\
0 \\
\vdots \\
1 \\
0 \\
\vdots \\
0
\end{array}\right]
$$

para $k \notin B$, onde 1 aparece na k-ésima coordenada. Se $\theta_{k}$ são os múltiplos positivos destas direções escolhidos pelo corte do Tŭ, então a inequação

$$
\sum_{k \notin B} \frac{1}{\theta_{k}} x_{k} \geqq 1
$$

define este corte. Note que $\sum_{k \notin B} \frac{1}{\theta_{k}} v_{k}=0$, e que os pontos da forma $x^{k}=v+\theta_{k} d^{k}$ satisfazem a restrição com igualdade. Mais ainda, se $d^{k}$ é uma direção ilimitada de crescimento da função, 
então podemos substituir $\frac{1}{\theta_{k}}$ na expressão acima por 0, evitanto gerar restrições mal-condicionadas numericamente. Este plano de corte é proposto por [Tŭ 64] como uma modificação do algorítmo (3) para o caso de um poliedro canônico não-degenerado.

Se uma solução básica viável $v$ de um poliedro canônico é degenerada, não é verdade que as direções definidas em (1.34) incluem todas as direções de arestas saindo de $v$. O exemplo seguinte ilustra esta afirmação: se $P^{\prime}$ é dado por

$$
P^{\prime}=\left\{x \in \mathbb{R}^{3} \mid \begin{array}{ccccc}
x_{1} & & & \leqq \\
& x_{2} & & \leqq \\
& & x_{3} & \leqq & 1 \\
x_{1} & +x_{2} & +x_{3} & \leqq & 1 \\
& & x & \geqq
\end{array}\right\}
$$

o poliedro canônico associado é da forma $P=\left\{x \in \mathbb{R}^{7} \mid A x+I x^{r}=b, x \geqq 0\right\}$, e tem 4 restrições. A partir do vértice $v=(1,1,1)^{t}$ podemos completar a base com qualquer uma das variáveis residuais, e para cada base encontraremos apenas 2 direções viáveis associadas a variáveis nãobásicas. No entanto $v$ tem 4 vizinhos, $(0,1,0)^{t},(0,1,1)^{t},(1,0,0)^{t}$ e $(1,0,1)^{t}$.

Este é também um bom exemplo para desassociar o fenômeno de degenerescência da presença de restrições supérfluas no sistema, interpretação errada porém com certa divulgação.

Veremos adiante (4.2) que é possível iniciar o algoritmo de Tŭ-Zwart para o (CFA) com um poliedro não-degenerado limitado dado por um sistema de $n^{2}$ equações e $(2 m+1) n$ variáveis. Porém à medida que vão sendo introduzidas novas equações (cortes de Tŭ̌) poderemos eventualmente atingir um sistema degenerado. Algumas maneiras de lidar com este problema são apresentadas na seção (4.2).

A exigência do poliedro ser limitado pode ser facilmente satisfeita no nosso problema, pois a função objetivo é crescente; isto significa por um lado que o problema tem solução ótima e por outro que se $v \leqq M$ para todo vértice $v$, não é interessante procurar uma solução entre os fluxos que não satisfazem $f \leqq M$. Como veremos na seção (3.3), existem algumas limitações naturais para os vértices do poliedro. Desta forma, poderemos introduzir uma restrição do tipo $\|f\|_{1} \leqq\|M\|_{1}$ (que é linear), tornando o poliedro limitado.

Já a necessidade de extensão da função côncava para todo o $\mathbb{R}^{m}$ é impossibilitada pelo mau comportamento da derivada da $D^{*}(\cdot)$ quando algum $f_{e}$ tende a 0 (ver proposição (2.19)). A única extensão côncava da $D^{*}(\cdot)$ fora do ortante positivo $\mathbb{R}_{+}^{m}$ seria portanto fazer $D^{*}(f)=-\infty$, $\forall f \notin \mathbb{R}_{+}^{m}$, o que introduz dificuldades como descontinuidade na fronteira. Trabalharemos então sempre no ortante positivo: ao explorar as direções de arestas a partir de um vértice nos restringiremos ao conjunto original de definição da $D^{*}(\cdot)$. 


\section{Capítulo 3}

\section{Estudo do Poliedro de Fluxos Multicomodidade}

Neste capítulo estudaremos a hierarquia de poliedros apresentada na seção (2.2.2). Nosso objetivo final é estarmos aptos a aplicar uma estratégia do tipo Tǔ̆-Zwart ao poliedro dos fluxos multicomodidade $\mathcal{F}$, e para isso iremos estabelecer uma condição necessária para os vértices deste poliedro, bem como uma estratégia de cômputo das arestas a partir de um vértice qualquer.

Uma noção importante é a de grafo suporte de um fluxo, que intuitivamente pode ser visto como o grafo, cujas arestas estão associadas a componentes não-nulas daquele fluxo.

Definição 3.1 (Grafo Suporte) Definimos $o$ grafo suporte de $f^{r s} \in \vec{F}^{r s}$ por $\vec{G}_{f}^{r s}=\left(V, \vec{E}^{\prime}\right)$ onde $\vec{E}^{\prime}=\left\{e \in \vec{E} \mid f_{e}^{r s}>0\right\}$.

Se $f^{r}=\sum_{s \neq r} f^{r s}$ definimos $\vec{G}_{f}^{r}=\left(V, \vec{E}^{\prime}\right)$ onde $\vec{E}^{\prime}=\left\{e \in \vec{E} \mid f_{e}^{r}>0\right\}$. Também usaremos $\vec{G}_{f}^{r s}$ para denotar os suportes das parcelas de $f^{r}$.

Se $f=\sum_{r \in V} f^{r}=\sum_{r \in V} \sum_{s \neq r} f^{r s} \in \overrightarrow{\mathcal{F}}$ definimos $\vec{G}_{f}=\left(V, \vec{E}^{\prime}\right)$ onde $\vec{E}^{\prime}=\left\{e \in \vec{E} \mid f_{e}>0\right\}$. Também usaremos $\vec{G}_{f}^{r}$ e $\vec{G}_{f}^{r s}$ para denotar os suportes das parcelas de $f^{r}$.

Analogamente definimos o suporte para os fluxos nos poliedros projetados, $F^{r s}$, $F^{r}$ e $\mathcal{F}$, denotando-os por $G_{f}^{r s}, G_{f}^{r} e G_{f}$.

\subsection{Fluxos com Uma Origem e Um Destino: $\vec{F}^{r s}$}

Vimos na seção (2.2.2) a definição do poliedro $\vec{F}^{r s}$. Estabeleceremos agora alguns resultados: 
Proposição 3.2 (Representação canônica de $\vec{F}^{r s}$ ) $O$ poliedro $\vec{F}^{r s}$ pode ser representado por

$$
\vec{F}^{r s}=\left\{\left(f_{k l}^{r s}\right) \in \mathbb{R}_{+}^{2 m} \mid \sum_{(k, l) \in \delta^{+}(k)} f_{k l}^{r s}-\sum_{(l, k) \in \delta^{-}(k)} f_{l k}^{r s}=b_{k}, k=1, \ldots, n-1\right\},
$$

onde

$$
b_{k}=\left\{\begin{aligned}
q_{r s} & \text { se } k=r \\
-q_{r s} & \text { se } k=s \\
0 & \text { se } k \neq r, s
\end{aligned}\right.
$$

Prova : O resultado é imediato a partir da definição (2.2), notando que para quaisquer $r$ e $s$, a última equação (associada ao nó rotulado como $k=n$ ) pode ser obtida somando-se todas as anteriores e trocando o sinal do resultado.

Proposição 3.3 (Caracterização de vértices e do cone de $\vec{F}^{r s}$ ) O conjunto de vértices de $\vec{F}^{r s}$ é dado por

$$
V=\left\{f^{r s} \in \vec{F}^{r s} \mid \vec{G}_{f}^{r s} \text { é um caminho dirigido de } r \text { a } s\right\} \neq \emptyset ;
$$

O cone associado ao poliedro $\vec{F}^{r s}$ é

$$
C=\left\{h \in \mathbb{R}_{+}^{2 m} \mid h=\sum_{i=1}^{k} \lambda_{i} h^{i}, \lambda \geqq 0 e h^{i} \in C^{\prime}\right\},
$$

onde

$$
C^{\prime}=\left\{h \in \mathbb{R}_{+}^{2 m} \mid \vec{G}_{h} \text { é um ciclo dirigido em } \vec{G}\right\}
$$

Prova: Da proposição (1.39) (usando $b_{r}=q_{r s}, b_{s}=-q_{r s}$, e $b_{j}=0, \forall j \neq r, s$ ), e (1.15) sabemos que $V\left(\vec{F}^{r s}\right) \subset V$, e $C\left(\vec{F}^{r s}\right) \subset C$.

Para notar que todo caminho corresponde a um vértice, seja $f^{r s} \in \vec{F}^{r s}$ tal que $G_{f}^{r s}$ é um caminho de $r$ a $s$. Basta ver que existe um vetor $a$, dado por

$$
a_{e}= \begin{cases}0 & \text { se } f_{e}^{r s}>0 \\ 1 & \text { caso contrário }\end{cases}
$$

tal que $f^{r s}$ é o único minimizador da função $\langle a, x\rangle$, logo pela proposição (1.31) temos que $f^{r s} \in V\left(\vec{F}^{r s}\right)$.

Note que $V\left(\vec{F}^{r s}\right) \neq \emptyset$ pois $G$ é conexo.

A inclusão $C \subset C\left(\vec{F}^{r s}\right)$ é imediata, pois os elementos de $C^{\prime}$ são circulações, e o conjunto das circulações formam um cone convexo. 
Note que a hipótese $q_{r s}>0$ é fundamental para a associação entre vértices e fluxos cujo suporte é um caminho dirigido, pois um suporte que é um caminho dirigido determina unicamente o fluxo associado. O caso $q_{r s}=0$ faria com que $V\left(\vec{F}^{r s}\right)=\{0\}$.

Podemos a partir da proposição (3.3) estabelecer uma limitação para os vértices de $\vec{F}^{r s}$ :

Proposição 3.4 (Limitação para os vértices de $\vec{F}^{r s}$ ) Cada vértice $v^{r s} \in V\left(\vec{F}^{r s}\right)$ satisfaz

$$
\sum_{e \in \vec{E}} v_{e}^{r s} \leqq(n-1) q_{r s}
$$

Prova : Basta notar que qualquer caminho de $r$ a $s$ tem no máximo $n-1$ arcos, e um vértice $v^{r s}$ associado ao caminho $\Pi^{r s}$ transporta $q_{r s}$ unidades de fluxo em cada um dos arco deste caminho.

\subsubsection{Descrição de $F^{r s}$ por Cortes}

Lembremos que a definição de $F^{r s}(2.5)$ utiliza uma projeção que consiste em somar as quantidades de fluxo que passam nos arcos $(k, l)$ e $(l, k)$. Esta projeção é uma transformação linear, o que nos permite utilizar o resultado (1.19) para a caracterização de vértices e raios do cone. Antes porém precisamos enunciar a seguinte definição:

Definição 3.5 ( $q_{r s}$-caminho) Dizemos que um fluxo $f^{r s} \in F^{r s}$ é um $q_{r s}$-caminho se $G_{f}^{r s}$ é um caminho de $r$ a s passando pelas arestas $e_{0}, e_{1}, \ldots, e_{k}$ e $f_{e_{i}}^{r s}=q_{r s}, \forall i=1, \ldots, k$.

É fácil notar que os $q_{r s}$-caminhos acima definidos estão em correspondência biunívoca com os vértices do poliedro $\vec{F}^{r s}$. Note que esta definição pressupõe a hipótese $q_{r s}>0$.

Proposição 3.6 (Caracterização de vértices e do cone de $F^{r s}$ ) $O$ conjunto de vértices de $F^{r s}$ é dado por

$$
V\left(F^{r s}\right)=\left\{f^{r s} \in F^{r s} \mid f^{r s} \text { é um } q_{r s} \text {-caminho }\right\} \neq \emptyset
$$

$O$ cone associado a $F^{r s}$ é $C\left(F^{r s}\right)=\mathbb{R}_{+}^{m}$, e portanto $\operatorname{dim}\left(F^{r s}\right)=m$.

Prova : Notemos inicialmente que, usando (1.19), temos que $C\left(F^{r s}\right)=\mathbb{R}_{+}^{m}$. Esta conclusão vem do fato que cada ciclo $i \longrightarrow j \longrightarrow i$, onde $h_{i j}=h_{j i}=\delta>0$ está no cone de $\vec{F}^{r s}$, e a projeção deste ciclo é $i-j \operatorname{com} h_{i j}=2 \delta$.

Mais ainda, a projeção de qualquer vértice de $\vec{F}^{r s}$ é um $q_{r s}$-caminho. Usando novamente (1.19) concluimos que

$$
V\left(F^{r s}\right) \subset\left\{f^{r s} \in F^{r s} \mid f^{r s} \text { é um } q_{r s} \text {-caminho }\right\} .
$$


Para verificar que todos os $q_{r s}$-caminhos são vértices de $F^{r s}$, basta notar que estes são solução ótima única de $\min \langle a, x\rangle$ neste poliedro, onde

$$
a_{e}= \begin{cases}1, & \text { se } e \text { está no } q_{r s} \text {-caminho; } \\ n, & \text { para os outros arcos. }\end{cases}
$$

Note ainda que $V\left(F^{r s}\right) \neq \emptyset$ pois $G$ é conexo.

Da caracterização (3.6) segue um interessante corolário:

Corolário 3.7 (Integralidade dos vértices de $F^{r s}$ ) Suponha que $q_{r s} \in \mathbb{N}$. Então todo vértice $f$ de $F^{r s}$ satisfaz $f \in \mathbb{N}^{m}$.

Note que os vértices de $\vec{F}^{r s}$ e $F^{r s}$ estão em correspondência biunívoca, portanto podemos tratar os fluxos $f^{r s} \in V\left(F^{r s}\right)$ como fluxos orientados (caminhos orientados) sempre que isto for conveniente.

Gostaríamos de poder obter também para este poliedro projetado uma representação da forma $A x \leqq b$. Com o uso da proposição (1.40) podemos obter o seguinte resultado:

Proposição 3.8 (Representação de $F^{r s}$ por Cortes) Sejam $S_{1}, S_{2}, \ldots, S_{k}$ todos os $s u b$ conjuntos de $V$ tais que $r \in S_{i}, s \in V \backslash S_{i}$ e $\delta\left(S_{i}\right)$ é um corte minimal no grafo $G$. Então

$$
F^{r s}=\left\{x \in \mathbb{R}_{+}^{m} \mid \sum_{e \in \delta\left(S_{i}\right)} x_{e} \geqq q_{r s}, i=1, \ldots, k\right\} ;
$$

Mais ainda, todas as desigualdades acima definem facetas do poliedro $F^{r s}$.

Prova : Seja $x \in F^{r s}$, e seja $f^{r s} \in \vec{F}^{r s}$ tal que $x$ é a projeção de $f^{r s}$. Então para cada $S_{i}$, somando as equações de (3.2) para $j \in S_{i}$, vemos que $f^{r s}$ satisfaz, para $i=1, \ldots, k$ :

$$
\sum_{e \in \delta^{+}\left(S_{i}\right)} f_{e}^{r s}-\sum_{e \in \delta^{-}\left(S_{i}\right)} f_{e}^{r s}=q_{r s}
$$

Então, para cada $i=1, \ldots, k$ :

$$
\begin{aligned}
\sum_{e \in \delta\left(S_{i}\right)} x_{e} & =\sum_{e \in \delta^{+}\left(S_{i}\right)} f_{e}^{r s}+\sum_{e \in \delta^{-}\left(S_{i}\right)} f_{e}^{r s} \\
& \geqq \sum_{e \in \delta^{+}\left(S_{i}\right)} f_{e}^{r s}-\sum_{e \in \delta^{-}}\left(S_{i}\right) f_{e}^{r s} \\
& =q_{r s} .
\end{aligned}
$$

Para provar a inclusão contrária, seja $x \in \mathbb{R}_{+}^{m}$ satisfazendo cada uma das restrições associadas a $S_{i}$, para $i=1, \ldots, k$. Considere o vetor $b$ dado por $b_{r}=q_{r s}, b_{s}=-q_{r s}$, e 
$b_{j}=0, \forall j \neq r, s$. Considere o grafo dirigido $\vec{G}$ com capacidades nos arcos dadas por $u_{k l}=u_{l k}=x_{e}$, se $e=\{k, l\}$. Então $u$ satisfaz

$$
\sum_{e \in \delta^{+}\left(S_{i}\right)} u_{e} \geqq q_{r s}=\sum_{j \in S_{i}} b_{j}
$$

e aplicando (1.40) existe um fluxo $f^{r s} \in \vec{F}^{r s}$ tal que $f_{k l}^{r s} \leqq u_{k l}, \forall(k, l) \in \vec{E}$. Se existe algum ciclo unitário em $f^{r s}$, isto é, se existe algum par $(k, l)$ tal que $f_{k l}^{r s}>0$ e $f_{l k}^{r s}>0$, podemos subtrair $\delta=\min \left\{f_{k l}^{r s}, f_{l k}^{r s}\right\}$ de ambas as variáveis; após a eliminação de todos os possíveis ciclos unitários, teremos que $f_{k l}^{r s}+f_{l k}^{r s} \leqq x_{e}, \forall e=\{k, l\}$, de onde a projeção $p\left(f^{r s}\right)$ de $f^{r s}$ satisfará $p\left(f^{r s}\right) \leqq x$, e lembrando que $C\left(F^{r s}\right)=\mathbb{R}_{+}^{m}$, temos que $x \in F^{r s}$.

Para concluir que cada uma destas desigualdades é uma faceta, mostraremos que para cada $i$ existem $m+1$ pontos do poliedro $x^{0}, \ldots, x^{m}$ que satisfazem a restrição associada a $S_{i}$ com igualdade e tais que $\left\{x^{1}-x^{0}, \ldots, x^{m}-x^{0}\right\}$ é L.I., de onde concluiremos que $F^{r s} \cap\left\{\sum_{e \in \delta\left(S_{i}\right)} x_{e} \geqq q_{r s}\right\}$ tem dimensão $m$.

Tome um $i$ qualquer, e seja $\delta\left(S_{i}\right)=\left\{e_{0}, \ldots, e_{k}\right\}$. Para cada uma das arestas $e_{j}$, escolha um caminho qualquer de $r$ a $s$ passando por $e_{j}$ (se tal caminho não existisse, o corte $S_{i}$ não seria minimal); chame de $x^{j}$ o $q_{r s}$-caminho associado. Até agora temos $k$ pontos que satisfazem a restrição de $S_{i}$ com igualdade. Para cada aresta $e_{j} \notin \delta\left(S_{i}\right)$, podemos construir os pontos da forma $x^{j}=x^{0}+M e_{j}$ (abusando a notação e identificando a aresta $e_{j}$ com a direção $j$ da base canônica do $\mathbb{R}^{m}$, que é um raio do cone $C\left(F^{r s}\right)$ ), escolhendo um $M>0$ suficientemente grande de forma que $\left\{x^{0}, \ldots, x^{m}\right\}$ seja L.I. Cada um destes pontos satisfaz a restrição associada a $S_{i}$ com igualdade, de onde segue a tese.

\subsection{Fluxos com Uma Origem e Vários Destinos: $\vec{F}^{r}$}

Analogamente ao que foi feito na seção anterior, começaremos com alguns resultados em relação ao poliedro $\vec{F}^{r}$.

Proposição 3.9 (Representação canônica de $\vec{F}^{r}$ ) O poliedro $\vec{F}^{r}$ pode ser representado por

$$
\vec{F}^{r}=\left\{\left(f_{k l}^{r}\right) \in \mathbb{R}_{+}^{2 m} \mid \sum_{(k, l) \in \delta^{+}(k)} f_{k l}^{r}-\sum_{(l, k) \in \delta^{-}(k)} f_{l k}^{r}=b_{k}, k=1, \ldots, n-1\right\},
$$

onde

$$
b_{k}=\left\{\begin{aligned}
\sum_{i \in V, i \neq r} q_{r i} & \text { se } k=r \\
-q_{r k} & \text { se } k \neq r
\end{aligned}\right.
$$

Este sistema será representado a partir de agora como $\mathcal{J} f=b^{r}$. 
Prova : Notemos inicialmente que $\vec{F}^{r}=\sum_{s \neq r} \vec{F}^{r s}$, a partir da definição (2.3). A partir das representações canônicas dos poliedros $\vec{F}^{r s}$, é claro que

$$
\vec{F}^{r} \subset\left\{\left(f_{k l}^{r}\right) \in \mathbb{R}_{+}^{2 m} \mid \sum_{(k, l) \in \delta^{+}(k)} f_{k l}^{r}-\sum_{(l, k) \in \delta^{-}(k)} f_{l k}^{r}=b_{k}, k=1, \ldots, n-1\right\},
$$

pois a matriz do lado esquerdo é igual para todos os poliedros, e o lado direito de $\vec{F}^{r}$ é a soma dos lados direitos de $\vec{F}^{r s}$.

Para a outra inclusão, seja $f^{r}$ satisfazendo todas as restrições do enunciado. Pela proposição (1.39), existem $n_{i} q_{r i}$-caminhos $\Pi_{j}^{r i}$ de $r$ a $i$ (para cada $i$ tal que $b_{i}<0$ ) e $l$ ciclos dirigidos $C^{k}$ tais que

$$
f^{r}=\sum_{i \neq r} \sum_{j=1}^{n_{i}} \alpha_{j}^{i} \Pi_{j}^{r i}+\sum_{k=1}^{l} \beta_{k} C^{k}
$$

$\operatorname{com} \alpha_{j}^{i}, \beta_{k} \geqq 0, \sum_{j=1}^{n_{i}} \alpha_{j}^{i}=-b_{i}, \forall i \neq r$. Basta então notar que $\sum_{j=1}^{n_{i}} \alpha_{j}^{i} \Pi_{j}^{r i} \in \vec{F}^{r i}$ para cada $i$, e que $\sum_{k=1}^{l} \beta_{k} C^{k} \in C\left(\vec{F}^{r i}\right)$ para todo $i$, de onde $f^{r} \in \vec{F}^{r}$.

Antes de prosseguirmos, uma definição:

Definição 3.10 (Árvore dirigida com raiz) Uma árvore dirigida com raiz $r$ é uma árvore em $\vec{G}$ com a seguinte propriedade: para cada nó $k \neq r$ as arestas em $\vec{G}$ do caminho entre $r$ e $k$ formam um caminho dirigido de $r$ para $k$.

Proposição 3.11 (Caracterização de vértices e do cone de $\vec{F}^{r}$ ) O conjunto de vértices de $\vec{F}^{r}$ é dado por

$$
V\left(\vec{F}^{r}\right)=\left\{f^{r} \in \vec{F}^{r} \mid \vec{G}_{f}^{r} \text { é uma árvore dirigida com raiz } r\right\} \neq \emptyset ;
$$

O cone associado ao poliedro $\vec{F}^{r}$ é

$$
C\left(\vec{F}^{r}\right)=\left\{h \in \mathbb{R}_{+}^{2 m} \mid h=\sum_{i=1}^{k} \lambda_{k} h^{k}, \lambda \geqq 0, e h^{k} \in C^{\prime}\right\}
$$

onde

$$
C^{\prime}=\left\{h \in \mathbb{R}_{+}^{2 m} \mid G_{h} \text { é um ciclo dirigido em } \vec{G}\right\} \text {. }
$$

Prova : A prova é análoga à de (3.3), usando $b_{r}=\sum_{i \in V, i \neq r} q_{r i}$, e $b_{j}=-q_{r j}, \forall j \neq r$. Para notar que toda árvore dirigida com raiz $r$ corresponde a um vértice, dada $f^{r} \in \vec{F}^{r}$ uma árvore dirigida com raiz $r$, basta tomar $a$, dado por

$$
a_{e}= \begin{cases}0 & \text { se } f_{e}^{r}>0 \\ 1 & \text { caso contrário }\end{cases}
$$


e aplicar (1.31).

A conclusão $V\left(\vec{F}^{r}\right) \neq \emptyset$ é imediata, pois $G$ é conexo.

Uma conseqüência imediata é:

Corolário 3.12 (Integralidade dos vértices de $\vec{F}^{r}$ ) Suponha que $q_{r s} \in \mathbb{N}$ para todo $s \neq r$. Então todo vértice $f$ de $\vec{F}^{r}$ satisfaz $f \in \mathbb{N}^{2 m}$.

Novamente a hipótese $q_{r s}>0, \forall s \neq r$ é crucial para "traduzir" o conceito de vértice de $\vec{F}^{r}$ em função dos suportes $G_{f}^{r}$. Note que se algum $q_{r s}$ fosse 0, então todas as soluções básicas do sistema $\mathcal{J} f=b^{r}$ tais que $s$ não aparece no interior de um caminho, seriam degeneradas:

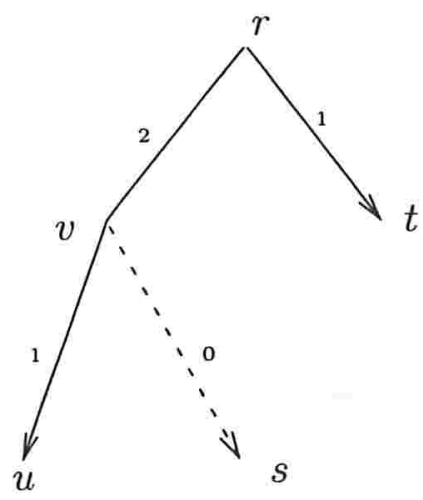

$$
\begin{aligned}
& q_{r s}=0 \\
& q_{r i}=1, \forall i \neq r, s
\end{aligned}
$$

\section{Exemplo de vértice degenerado $\left(q_{r s}=0\right)$}

Podemos a partir da proposição (3.11) estabelecer uma limitação para os vértices de $\vec{F}^{r}$ :

Proposição 3.13 (Limitação para os vértices de $\vec{F}^{r}$ ) Cada vértice $v^{r} \in V\left(\vec{F}^{r}\right)$ satisfaz

$$
\sum_{e \in \vec{E}} v_{e}^{r} \leqq(n-1) \sum_{s \neq r} q_{r s}
$$

Prova : Basta notar que qualquer qualquer árvore com raiz $r$ tem no máximo $n-1$ arcos, e um vértice $v^{r}$ associado a esta árvore transporta no máximo $\sum_{s \neq r} q_{r s}$ unidades de fluxo em cada um dos arcos deta árvore.

\subsubsection{Descrição de $F^{r}$ por Cortes}

Novamente a definição de $F^{r}(2.6)$ utiliza a projeção que consiste em somar as quantidades de fluxo que passam nos $\operatorname{arcos}(k, l)$ e $(l, k)$. Seguindo os passos da seção (3.1.1), forneceremos uma caracterização de vértices e raios do cone de $F^{r}$, utilizando (1.19). 
Definição 3.14 ( $q_{r}$-árvore) Dizemos que um fluxo $f^{r} \in F^{r}$ é uma $q_{r}$-árvore se $G_{f}^{r}$ é uma árvore e se vale a seguinte propriedade: removendo uma aresta qualquer e da árvore, e indicando por $K$ o conjunto de nós na componente conexa que não contém $r$, temos que $f_{e}^{r}=\sum_{k \in K} q_{r k}$.

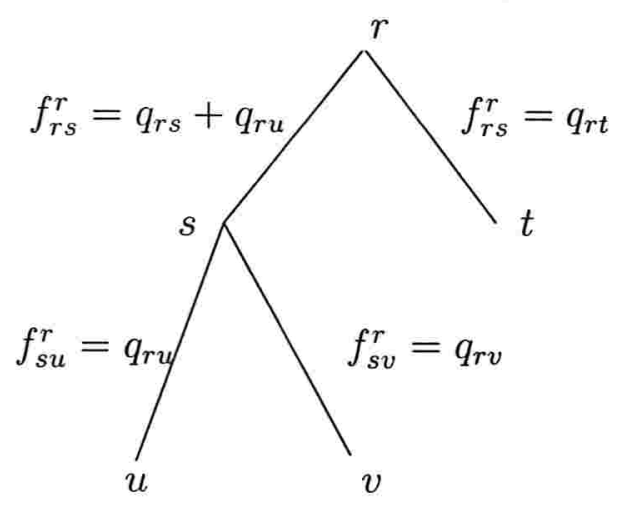

\section{Exemplo de $q_{r}$-árvore} $\vec{F}^{r s}$.

É fácil notar que as $q_{r}$-árvores estão em correspondência biunívoca com os vértices do poliedro

Proposição 3.15 (Caracterização de vértices e do cone de $F^{r}$ ) O conjunto de vértices de $F^{r}$ é dado por

$$
V\left(F^{r}\right)=\left\{f^{r} \in F^{r} \mid f^{r} \text { é uma } q_{r} \text {-árvore }\right\} \neq \emptyset ;
$$

$O$ cone associado a $F^{r}$ é $C\left(F^{r}\right)=\mathbb{R}_{+}^{m}$, e portanto $\operatorname{dim}\left(F^{r s}\right)=m$.

Prova : Notemos inicialmente que, usando (1.19), temos que $C\left(F^{r}\right)=\mathbb{R}_{+}^{m}$. O cone é igual ao do poliedro $F^{r s}$, pois $C\left(\vec{F}^{r}\right)=C\left(\vec{F}^{r s}\right)$.

Mais ainda, a projeção de qualquer vértice de $\vec{F}^{r}$ é uma $q_{r}$-árvore. Usando novamente (1.19) concluimos que

$$
V\left(F^{r}\right) \subset\left\{f^{r} \in F^{r} \mid f^{r} \text { é uma } q_{r} \text {-árvore }\right\} .
$$

Para verificar que todos as $q_{r s}$-árvores são vértices de $F^{r}$, basta notar que estas são solução ótima única de $\min \langle a, x\rangle$ neste poliedro, onde $a_{e}=1$ se $e$ está na $q_{r}$-árvore e $a_{e}=n$, para os outros arcos.

A conclusão $V\left(F^{r}\right) \neq \emptyset$ é imediata, pois $G$ é conexo.

Deste resultado segue imediatamente que:

Corolário 3.16 (Integralidade dos vértices de $F^{r}$ ) Suponha que $q_{\text {rs }} \in \mathbb{N}$ para todo $s \neq r$. Então todo vértice $f$ de $F^{r}$ satisfaz $f \in \mathbb{N}^{m}$. 
Note que os vértices de $\vec{F}^{r}$ e $F^{r}$ estão em correspondência biunívoca, portanto podemos tratar os fluxos $f^{r} \in V\left(F^{r}\right)$ como fluxos orientados (árvores orientadas) sempre que isto for conveniente.

Também para este poliedro projetado podemos obter uma representação da forma $A x \leqq b$, de maneira análoga à empregada em (3.8).

Proposição 3.17 (Representação de $F^{r}$ por Cortes) Sejam $S_{1}, S_{2}, \ldots, S_{k}$ todos os subconjuntos de $V$ tais que $r \in S_{i}, S_{i} \neq V$ e $\delta\left(S_{i}\right)$ é um corte minimal no grafo $G$. Então

$$
F^{r}=\left\{x \in \mathbb{R}_{+}^{m} \mid \sum_{e \in \delta\left(S_{i}\right)} x_{e} \geqq \sum_{s \in V \backslash S_{i}} q_{r s}, i=1, \ldots, k\right\}
$$

Mais ainda, todas as desigualdades acima definem facetas do poliedro $F^{r}$.

Prova : Seja $x \in F^{r}$, e seja $f^{r} \in \vec{F}^{r}$ tal que $x$ é a projeção de $f^{r}$. Então para cada $S_{i}$, somando as equações de (3.9) para $j \in S_{i}$, vemos que $f^{r}$ satisfaz, para $i=1, \ldots, k$ :

$$
\sum_{e \in \delta^{+}\left(S_{i}\right)} f_{e}^{r}-\sum_{e \in \delta^{-}\left(S_{i}\right)} f_{e}^{r}=\sum_{s \in V \backslash S_{i}} q_{r s}
$$

Então, para cada $i=1, \ldots, k$ :

$$
\begin{aligned}
\sum_{e \in \delta\left(S_{i}\right)} x_{e} & =\sum_{e \in \delta^{+}\left(S_{i}\right)} f_{e}^{r}+\sum_{e \in \delta^{-}\left(S_{i}\right)} f_{e}^{r} \\
& \geqq \sum_{e \in \delta^{+}\left(S_{i}\right)} f_{e}^{r}-\sum_{e \in \delta^{-}\left(S_{i}\right)} f_{e}^{r} \\
& =\sum_{s \in V \backslash S_{i}} q_{r s} .
\end{aligned}
$$

Para provar a inclusão contrária, seja $x \in \mathbb{R}_{+}^{m}$ satisfazendo cada uma das restrições associadas a $S_{i}$, para $i=1, \ldots, k$. Considere o vetor $b$ de (3.9) e considere o grafo dirigido $\vec{G}$ com capacidades nos arcos dadas por $u_{k l}=u_{l k}=x_{e}$, se $e=\{k, l\}$. Então $u$ satisfaz

$$
\sum_{e \in \delta^{+}\left(S_{i}\right)} u_{e} \geqq \sum_{s \in V \backslash S_{i}} q_{r s}=\sum_{j \in S_{i}} b_{j}
$$

e aplicando (1.40) existe um fluxo $f^{r} \in \vec{F}^{r}$ tal que $f_{k l}^{r} \leqq u_{k l}, \forall(k, l) \in \vec{E}$. Subtraindo todos os ciclos unitários da mesma maneira que foi feito na prova de (3.8), teremos que $f_{k l}^{r}+f_{l k}^{r} \leqq x_{e}, \forall e=\{k, l\}$, de onde a projeção $p\left(f^{r}\right)$ de $f^{r}$ satisfará $p\left(f^{r}\right) \leqq x$, e lembrando que $C\left(F^{r}\right)=\mathbb{R}_{+}^{m}$, temos que $x \in F^{r}$.

Para concluir que cada uma destas desigualdades é uma faceta, como em (3.8), mostraremos que para cada $i$ existem $m+1$ pontos do poliedro $x^{0}, \ldots, x^{m}$ que satisfazem a restrição associada a $S_{i}$ com igualdade e tais que $\left\{x^{1}-x^{0}, \ldots, x^{m}-x^{0}\right\}$ é L.I.

Tome um $i$ qualquer, e seja $\delta\left(S_{i}\right)=\left\{e_{0}, \ldots, e_{k}\right\}$. Para cada uma das arestas $e_{j}$, escolha uma árvore qualquer que passe em $e_{j}$ mas não passe nas outras arestas do corte (se tal árvore não existisse, o corte $S_{i}$ não seria minimal); chame de $x^{j}$ a $q_{r}$-árvore associada. Até agora temos $k$ pontos que satisfazem a restrição de $S_{i}$ com igualdade. Para cada aresta $e_{j} \notin \delta\left(S_{i}\right)$, podemos construir os pontos da forma $x^{j}=x^{0}+M e_{j}$, escolhendo um $M>0$ suficientemente grande de forma que $\left\{x^{0}, \ldots, x^{m}\right\}$ seja L.I. Cada um destes pontos satisfaz a restrição associada a $S_{i}$ com igualdade, de onde segue a tese. 


\subsection{Fluxos com Várias Origens e Destinos: $\overrightarrow{\mathcal{F}}$ e $\mathcal{F}$}

Quando consideramos fluxos multicomodidade, algumas dificuldades naturalmente aparecem. A proposição (1.39) se aplica apenas a fluxos com respeito $a b$, onde $b$ é um vetor com a propriedade de que apenas um $b_{r}$ é estritamente positivo ( $r$ é a origem do fluxo). Crucial na demonstração daquela proposição era o fato de que, para qualquer fluxo $f_{k l}^{r}=\sum_{s \neq r} f_{k l}^{r s}$ sabíamos que a origem de todas as parcelas deste fluxo era um único nó $r$, mesmo que o valor de cada parcela não fosse conhecido a priori (como nas definições (3.5) e (3.14)).

A abordagem por projeção, utilizando a proposição (1.19) não nos permite concluir qualquer propriedade especial dos suportes para vértices de $\overrightarrow{\mathcal{F}}$. Como veremos adiante, $f \in \overrightarrow{\mathcal{F}}$ pode ser vértice mesmo tendo suporte $\vec{G}_{f} \equiv \vec{G}$.

No entanto conhecemos a forma canônica do poliedro $\vec{F}^{1} \times \vec{F}^{2} \times \cdots \times \vec{F}^{n}$, o produto cartesiano dos poliedros $\vec{F}^{r}$; esta representação é conseqüência imediata da proposição (1.18), e será usada na descrição do algoritmo de Tư̆-Zwart aplicado ao (CFA), seção (4.2).

Proposição 3.18 (Representação canônica de $\vec{F}^{1} \times \vec{F}^{2} \times \cdots \times \vec{F}^{n}$ )

$O$ poliedro $\vec{F}^{1} \times \vec{F}^{2} \times \cdots \times \vec{F}^{n}$ pode ser representado por

$$
\left\{\left(f^{1}, f^{2}, \ldots, f^{n}\right) \in \mathbb{R}_{+}^{2 m n} \mid \begin{array}{ccccc}
\mathcal{J} f^{1} & & & & \\
& \mathcal{J} f^{2} & & b^{1} \\
& & & b^{2} \\
& \ddots & \vdots & \vdots \\
& & \mathcal{J} f^{n} & = & b^{n} \\
& \left(f^{1}, f^{2}, \ldots, f^{n}\right)^{t} & \geqq
\end{array}\right\}
$$

onde a matriz $\mathcal{J}$ foi definida em (3.9) e $b^{r}$ é o lado direito da forma canônica de $\vec{F}^{r}$, isto é,

$$
b_{k}^{r}=\left\{\begin{aligned}
\sum_{s \neq r} q_{r s} & \text { se } k=r \\
-q_{r s} & \text { se } k \neq r .
\end{aligned}\right.
$$

Com o auxílio da proposição (1.18) podemos trivialmente afirmar:

Proposição 3.19 (Caracterização de vértices e do cone de $\vec{F}^{1} \times \vec{F}^{2} \times \cdots \times \vec{F}^{n}$ ) $O$ conjunto de vértices de $\vec{F}^{1} \times \vec{F}^{2} \times \cdots \times \vec{F}^{n}$ é dado por

$$
\left\{\left(f^{1}, f^{2}, \ldots, f^{n}\right) \mid f^{r} \in \vec{F}^{r} \text { e } \vec{G}_{f}^{r} \text { é uma árvore dirigida com raiz } r \text {, para } r \in V\right\} \text {. }
$$

O cone associado a este poliedro é

$$
C\left(\vec{F}^{1} \times \cdots \times \vec{F}^{n}\right)=C\left(\vec{F}^{1}\right) \times \cdots \times C\left(\vec{F}^{n}\right) .
$$


Resgatando a discussão sobre limitações para os vértices do poliedro $\vec{F}^{1} \times \vec{F}^{2} \times \cdots \times \vec{F}^{n}$, de (3.4) e (3.13), temos automaticamente que, se $\left(f^{1}, \ldots, f^{n}\right)$ é um vértice deste poliedro, então

$$
\sum_{e \in \vec{E}} f_{e}^{r} \leqq(n-1) \sum_{s \neq r} q_{r s}, \forall r \in V
$$

Além disso, podemos afirmar:

Proposição 3.20 (Limitação para os vértices de $\left.\vec{F}^{1} \times \vec{F}^{2} \times \cdots \times \vec{F}^{n}\right)$ Se $\left(f^{1}, \ldots, f^{n}\right)$ é um vértice do poliedro $\vec{F}^{1} \times \vec{F}^{2} \times \cdots \times \vec{F}^{n}$, então

$$
\begin{gathered}
\sum_{e \in \vec{E}} f_{e}^{r} \leqq(n-1) \sum_{s \neq r} q_{r s}, \forall r \in V . \\
\sum_{r \in V} \sum_{e \in \vec{E}} f_{e}^{r} \leqq m \sum_{r \in V} \sum_{s \neq r} q_{r s} .
\end{gathered}
$$

Prova : A primeira afirmação é conseqüência de (3.13). Para a segunda, basta notar que cada $f^{r}$ é uma árvore. Cada arco do grafo estará presente, no máximo, em todos os caminhos $\vec{G}_{f}^{r s}$, de onde

$$
\sum_{r \in V}\left(f_{k l}^{r}+f_{l k}^{r} \leqq \sum_{r \in V} \sum_{s \neq r} q_{r s}\right.
$$

note que não poderia haver um arco tal que $f_{k l}^{r}>0$ e $f_{l k}^{r}>0$, pois $f^{r}$ é uma árvore. Somando-se a desigualdade para todos os $\operatorname{arcos}\{k, l\}$ não-dirigidos, vem a desigualdade do enunciado.

Note que a proposição anterior fornece duas maneiras de "limitar" o poliedro $\vec{F}^{1} \times \vec{F}^{2} \times \cdots \times \vec{F}^{n}$. A primeira é incluir todas as desigualdades

$$
\sum_{e \in \vec{E}} f_{e}^{r} \leqq(n-1) \sum_{s \neq r} q_{r s}
$$

para cada $r \in V$. A outra é incluir apenas uma desigualdade:

$$
\sum_{r \in V} \sum_{e \in \vec{E}} f_{e}^{r} \leqq m \sum_{r \in V} \sum_{s \neq r} q_{r s}
$$

Esta desigualdade, porém, apresenta a possibilidade do coeficiente do lado direito ser muito grande; as conseqüências disto serão discutidas na seção sobre a implementação do algorítmo de Tŭ-Zwart para o poliedro $\vec{F}^{1} \times \vec{F}^{2} \times \cdots \times \vec{F}^{n}$, na seção (4.2).

Com relação ao poliedro $\overrightarrow{\mathcal{F}}$, uma conseqüência interessante de (1.19) é:

Corolário 3.21 (Integralidade dos vértices de $\overrightarrow{\mathcal{F}}$ ) Suponha que $q_{r s} \in \mathbb{N}$ para todo par $(r, s)$. Então todo vértice $f$ de $\overrightarrow{\mathcal{F}}$ satisfaz $f \in \mathbb{N}^{2 m}$. 
Prova : Se $f \in V(\overrightarrow{\mathcal{F}})$, então por (1.19) existem $\left\{f^{r}\right\}_{r \in V}$ vértices em $\vec{F}^{r}$, tais que $f=\sum_{r \in V} f^{r}$. Mas por (3.12), cada $f^{r} \in \mathbb{N}^{2 m}$, logo $f \in \mathbb{N}^{2 m}$.

Para $\overrightarrow{\mathcal{F}}$, podemos estabelecer uma condição necessária para que $f \in V(\overrightarrow{\mathcal{F}})$, além de podermos trivialmente caracterizar o cone $C(\overrightarrow{\mathcal{F}})$ :

Proposição 3.22 (Condição necessária para $f \in V(\overrightarrow{\mathcal{F}})$ e cone de $\overrightarrow{\mathcal{F}})$ Se $f \in V(\overrightarrow{\mathcal{F}})$, então para quaisquer $\left\{f^{r}\right\}_{r \in V}$ com $f^{r} \in \vec{F}^{r}$ tais que $f=\sum_{r \in V} f^{r}$, temos que $f^{r} \in V\left(\vec{F}^{r}\right)$. $O$ cone de $\overrightarrow{\mathcal{F}}$ é dado por

$$
C(\overrightarrow{\mathcal{F}})=C\left(\vec{F}^{r}\right)=C\left(\vec{F}^{r s}\right), \forall r \in V, \forall s \neq r
$$

Prova : Sejam $\left\{f^{r}\right\}_{r \in V}$ com $f^{r} \in \vec{F}^{r}$ tais que $f=\sum_{r \in V} f^{r}$ e, sem perda de generalidade, suponha por contradição que $f^{1}$ não é vértice no poliedro $\vec{F}^{1}$; então existem $\hat{f}^{1} \in F^{r}$ e $\tilde{f}^{1} \in \vec{F}^{r}$, com $\hat{f}^{1} \neq \tilde{f}^{1}$ e tais que $f^{1}=\frac{1}{2} \hat{f}^{1}+\frac{1}{2} \tilde{f}^{1}$.

Conseqüentemente,

$$
f=\frac{1}{2}\left(\hat{f}^{1}+f^{2}+\cdots+f^{n}\right)+\frac{1}{2}\left(\tilde{f}^{1}+f^{2}+\cdots+f^{n}\right),
$$

onde $\left(\hat{f}^{1}+f^{2}+\cdots+f^{n}\right) \neq\left(\tilde{f}^{1}+f^{2}+\cdots+f^{n}\right)$, o que não pode ocorrer já que $f$ é vértice de $\overrightarrow{\mathcal{F}}$.

O argumento para o cone é o mesmo de (3.3) e (3.11), seguindo imediatamente da proposição (1.19).

Note que o resultado (1.19) nos permite concluir se $f \in V(\overrightarrow{\mathcal{F}})$ então existem $\left\{f^{r}\right\}_{r \in V}$ vértices, tais que $f=\sum_{r \in V} f^{r}$. A proposição (3.22) afirma que quaisquer $\left\{f^{r}\right\}_{r \in V}$ tais que $f=\sum_{r \in V} f^{r}$ são vértices nos poliedros $\vec{F}^{r}$. Em geral não podemos afirmar que $f \in \overrightarrow{\mathcal{F}}$ tem somente uma decomposição em fluxos $\left\{f^{r}\right\}_{r \in V}$, ou em outros termos, que a pré-imagem de $f$ em relação à projeção que leva $\vec{F}^{1} \times \vec{F}^{2} \times \cdots \times \vec{F}^{n}$ em $\mathcal{F}$ se reduz a um ponto. Mas para $f \in V(\mathcal{F})$ ou $f \in V(\overrightarrow{\mathcal{F}})$ esta afirmação é verdadeira, como veremos em (3.32).

Observamos que a volta de (3.22) não é válida. O fluxo associado às árvores

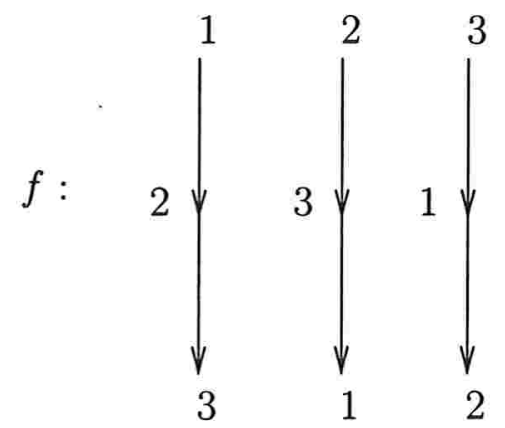

na topologia 


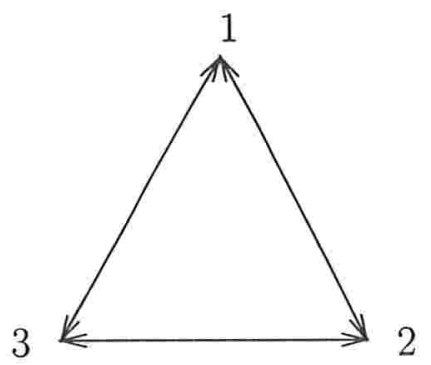

não é um vértice de $\overrightarrow{\mathcal{F}}$, pois $f=\frac{1}{3} f^{1}+\frac{1}{3} f^{2}+\frac{1}{3} f^{3}+h$ onde

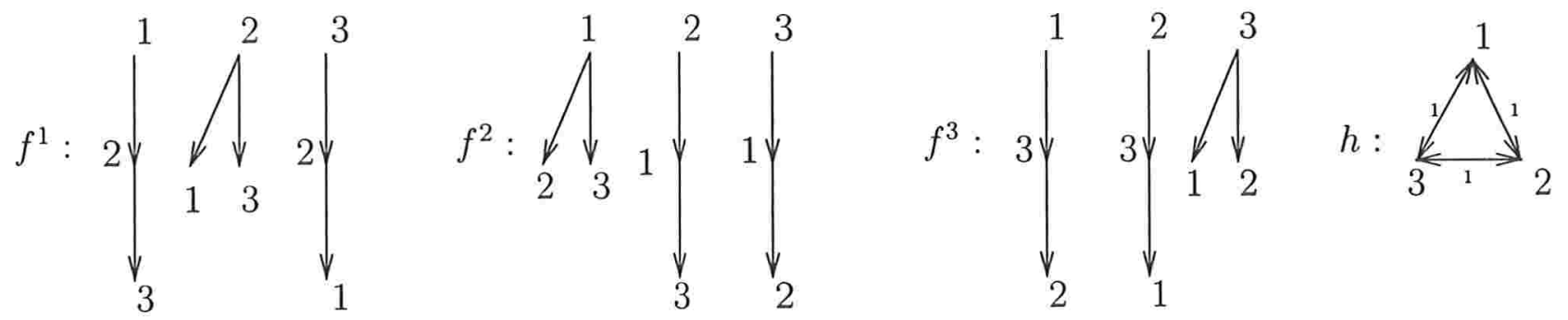

e $h \in C(\overrightarrow{\mathcal{F}})$.

Buscando uma melhor caracterização de $V(\overrightarrow{\mathcal{F}})$, que está associado (por (3.22)) a $n$ árvores, é natural procurar impôr relações entre estas árvores. Dada a interpretação de vértice como minimizador único de custos associados aos fluxos nos arcos, foi natural introduzir o conceito de fluxos coerentes:

Definição 3.23 (Fluxos coerentes em $\overrightarrow{\mathcal{F}}$ ) Um fluxo $f=\sum_{r \in V} f^{r} \in \overrightarrow{\mathcal{F}}$ é um fluxo coerente se cada $f^{r}$ é um vértice de $\vec{F}^{r}$ e para qualquer par de nós $(i, j)$ todos os caminhos dirigidos de $i$ a $j$ nas árvores $f^{1}, f^{2}, \ldots, f^{n}$ são idênticos, e todo caminho dirigido de $j$ a $i$ é o inverso do caminho de $i$ a $j$. Graficamente:
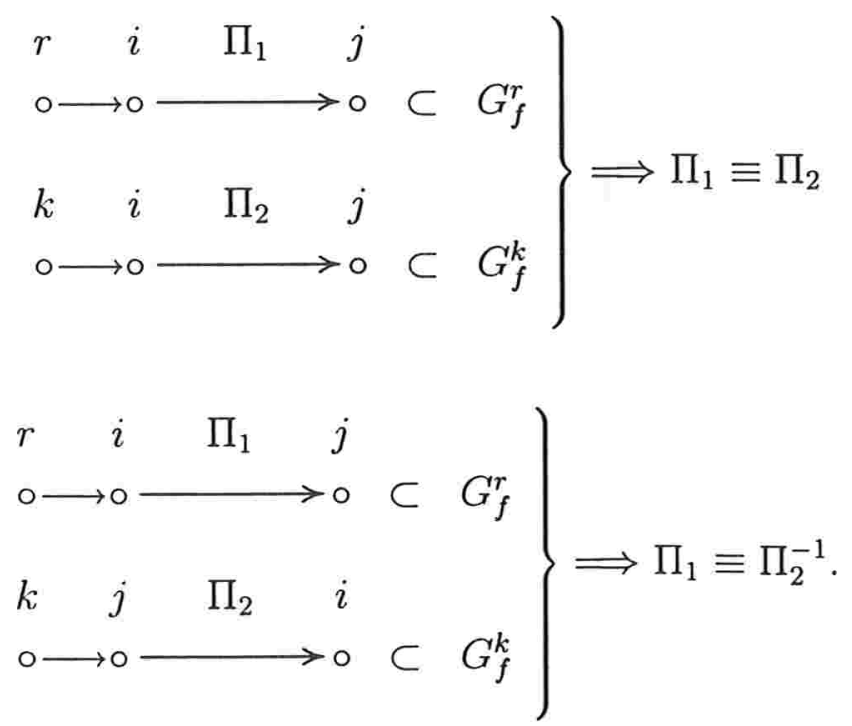
Note que a definição de fluxo coerente depende da representação, ou decomposição, do fluxo $f$ nas parcelas $f^{r}$.

Como todos os $q_{r s}$ são estritamente positivos, a cada fluxo coerente podemos associar um conjunto de caminhos $\Pi_{f}^{r s}$, cada um representando o único caminho "permitido" entre $i$ e $j$; além disso, se $G_{f}$ é o suporte de um fluxo coerente, e $(i, j)$ é um arco de $G_{f}$, então o caminho $\Pi_{f}^{i j}$ é

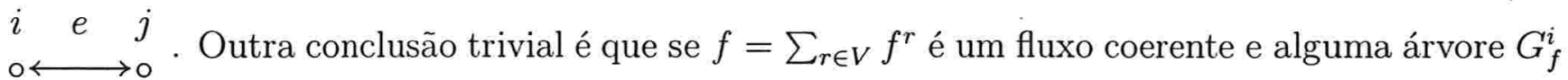
se reduz a um caminho dirigido começando em $i$, então todas as outras árvores utilizarão somente os arcos deste caminho ou os arcos reversos.

Note-se que o exemplo que motivou a última definição corresponde a um fluxo não-coerente. Tal fato é decorrência de:

Proposição 3.24 (Vértices de $\overrightarrow{\mathcal{F}}$ são fluxos coerentes) Se $f \in V(\overrightarrow{\mathcal{F}})$, então qualquer decomposição $f=\sum_{r \in V} f^{r}$ onde $f^{r} \in \vec{F}^{r}, \forall r$ é um fluxo coerente.

Prova : Se $f=\sum_{r \in V} f^{r} \operatorname{com} f^{r} \in \vec{F}^{r}, \forall r$ e $f \in V(\overrightarrow{\mathcal{F}})$, segue de (3.22) que cada $f^{r}$ é um vértice de $\vec{F}^{r}$, ou em outros termos que cada suporte $G_{f}^{r}$ é uma árvore dirigida com raiz $r$.

Portanto se $f=\sum_{r \in V} f^{r}$ não é um fluxo coerente então existe um par de nós $(i, j)$ e um par de árvores $\left(G_{f}^{r}, G_{f}^{s}\right)$ tais que

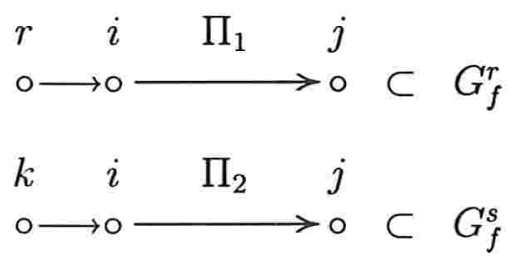

com $\Pi_{1} \neq \Pi_{2}$ (o caso em que um dos caminhos é de $i$ para $j$ e o outro é de $j$ para $i$ é análogo). Desta maneira, tomando $\varepsilon=\min \left\{q_{r j}, q_{s j}\right\}$, podemos definir os fluxos

$$
x_{e}^{r}=\left\{\begin{array}{ll}
f_{e}^{r}-\varepsilon & \forall e \in \Pi_{1} / \Pi_{2} \\
f_{e}^{r}+\varepsilon & \forall e \in \Pi_{2} / \Pi_{1} \\
f_{e}^{r} & \text { caso contrário, }
\end{array} \quad\left(x^{k}\right)=\left(f^{k}\right), \forall k \neq r\right.
$$

$\mathrm{e}$

$$
y_{e}^{s}=\left\{\begin{array}{ll}
f_{e}^{s}+\varepsilon & \forall e \in \Pi_{1} / \Pi_{2} \\
f_{e}^{s}-\varepsilon & \forall e \in \Pi_{2} / \Pi_{1} \\
f_{e}^{s} & \text { caso contrário. }
\end{array} \quad\left(y^{k}\right)=\left(f^{k}\right), \forall k \neq s\right.
$$

Note que $x=\sum_{k \in V} x^{k} \in \overrightarrow{\mathcal{F}}$ e $y=\sum_{k \in V} y^{k} \neq f \in \overrightarrow{\mathcal{F}}$. Como os caminhos $\Pi_{1}$ e $\Pi_{2}$ são diferentes, temos que $x \neq f$ e $y \neq f$, porém $f=\frac{1}{2} x+\frac{1}{2} y$, contradizendo o fato de $f$ ser vértice de $\overrightarrow{\mathcal{F}}$.

Isto conclui a prova. 
Infelizmente a condição necessária apresentada em (3.24) não é suficiente. Para verificar esta afirmação, incluímos na página seguinte um contra-exemplo.

Os fluxos $x$ e $y$ deste contra-exemplo são vértices do poliedro $\overrightarrow{\mathcal{F}}$ associado, como pôde ser verificado pela enumeração completa de $V(\overrightarrow{\mathcal{F}})$ (através da enumeração completa de todos os fluxos coerentes e eliminação daqueles que são combinação convexa dos outros). Isso mostra que um vértice pode ter como suporte o grafo $\vec{G}$.

No entanto chama a atenção o fato de existirem muito menos fluxos coerentes, e portanto muito menos vértices em $\overrightarrow{\mathcal{F}}$, do que vértices em $\vec{F}^{1} \times \vec{F}^{2} \times \cdots \times \vec{F}^{n}$, pois qualquer combinação de árvores dirigidas com raizes $1, \ldots, n$ está associada a um vértice deste poliedro, enquanto somente aquelas $n$-uplas de árvores dirigidas com a propriedade de coerência são eventuais "candidatas" a vértices de $\overrightarrow{\mathcal{F}}$.

O poliedro $\mathcal{F}$ "herda" várias das propriedades de $\overrightarrow{\mathcal{F}}$ por projeção:

Proposição 3.25 (Condição necessária para $f \in V(\mathcal{F})$ e cone de $\mathcal{F}$ ) Se $f \in V(\mathcal{F})$, então para quaisquer $\left\{f^{r}\right\}_{r \in V}$ com $f^{r} \in F^{r}$ tais que $f=\sum_{i \in V} f^{r}$, temos que $f^{r} \in V\left(F^{r}\right)$.

$O$ cone de $\mathcal{F}$ é dado por

$$
C(\mathcal{F})=C\left(F^{r}\right)=C\left(F^{r s}\right)=\mathbb{R}_{+}^{m}, \forall r \in V, \forall s \neq r .
$$

Prova : Idêntica à de (3.22).

Corolário 3.26 (Integralidade dos vértices de $\mathcal{F}$ ) Suponha que $q_{\text {rs }} \in \mathbb{N}$ para todo par $(r, s)$. Então todo vértice $f$ de $\mathcal{F}$ satisfaz $f \in \mathbb{N}^{m}$.

As noções de caminhos dirigidos descendentes nas árvores $f^{r} \in V\left(\vec{F}^{r}\right)$, e a definição de fluxos (dirigidos) coerentes, são adaptadas no caso não-dirigido como segue:

Definição 3.27 (Caminhos válidos) Seja $f^{r} \in V\left(F^{r}\right)$ uma $q_{r}$-árvore. Dizemos que um caminho de $i$ a $j$ é válido em $f^{r}$ se este caminho é um subgrafo de $G_{f}^{r}$, e se ou o caminho de $r$ a $i$ em $G_{f}^{r}$ passa por $j$, ou o caminho de $r$ a $j$ em $G_{f}^{r}$ passa por $i$.

Em outros termos, os caminhos válidos em $f^{r}$ correspondem aos caminhos dirigidos da árvore $\bar{f}^{r} \in \vec{F}^{r}$ associada a $f^{r}$.

Definição 3.28 (Fluxos coerentes em $\mathcal{F}$ ) Um fluxo $f=\sum_{r \in V} f^{r} \in \mathcal{F}$ é um fluxo coerente se cada $f^{r}$ é um vértice de $F^{r}$ e para qualquer par de nós (i,j) todos os caminhos válidos de $i$ a $j$ nas $q_{r}$-árvores $f^{1}, f^{2}, \ldots, f^{n}$ são idênticos.

Novamente poderemos associar a cada fluxo coerente um conjunto de caminhos (não-dirigidos) $\Pi_{f}^{r s}$ representando os caminhos válidos que aparecem nas $q_{r}$-árvores. 


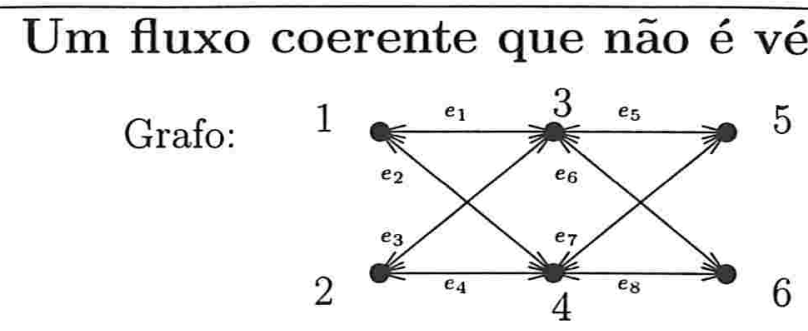

Ordem das arestas: $(13,14,23,24,35,36,45,46,31,41,32,42,53,63,54,64)$

Demandas: $q_{r s}=1, \forall r, s, r \neq s$

$$
\mathbf{z}=\frac{1}{2} x+\frac{1}{2} y
$$

Fluxo coerente $x=(2,3,2,3,1,4,4,3,2,3,2,3,1,4,4,3)^{t}$ :
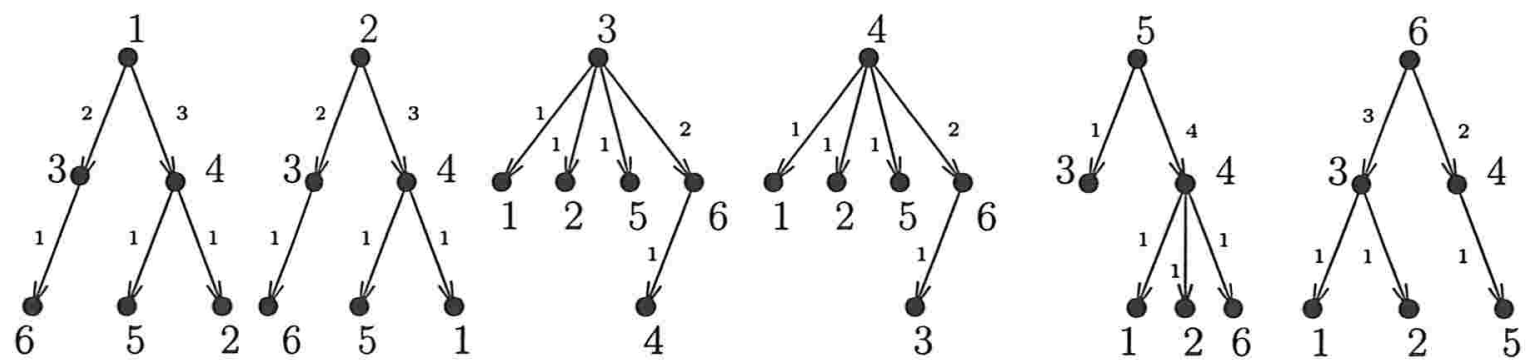

Fluxo coerente $y=(2,3,2,3,3,2,2,5,2,3,2,3,3,2,2,5)^{t}$ :
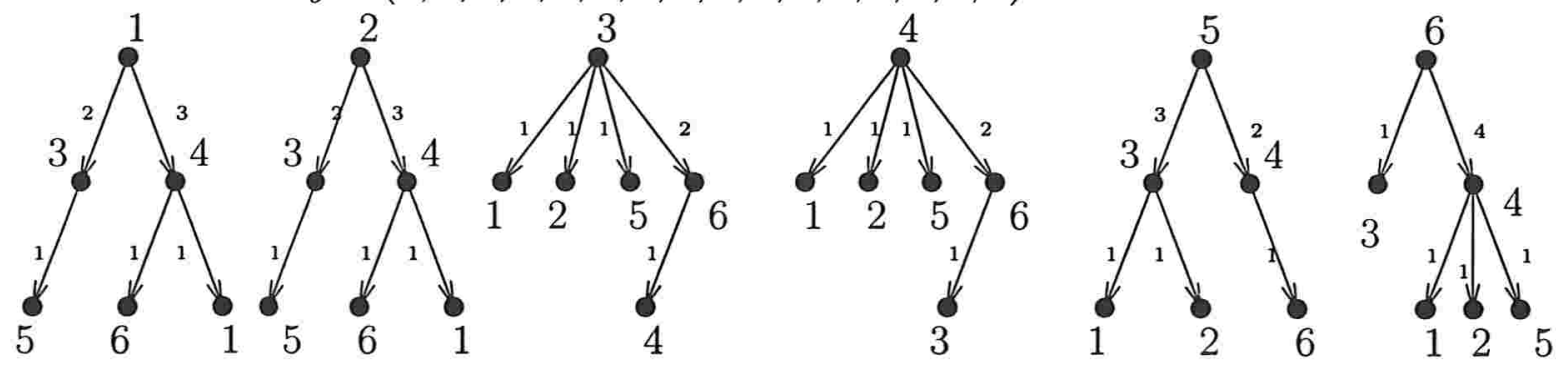

Fluxo coerente $z=(2,3,2,3,2,3,3,4,2,3,2,3,2,3,3,4)^{t}$ :
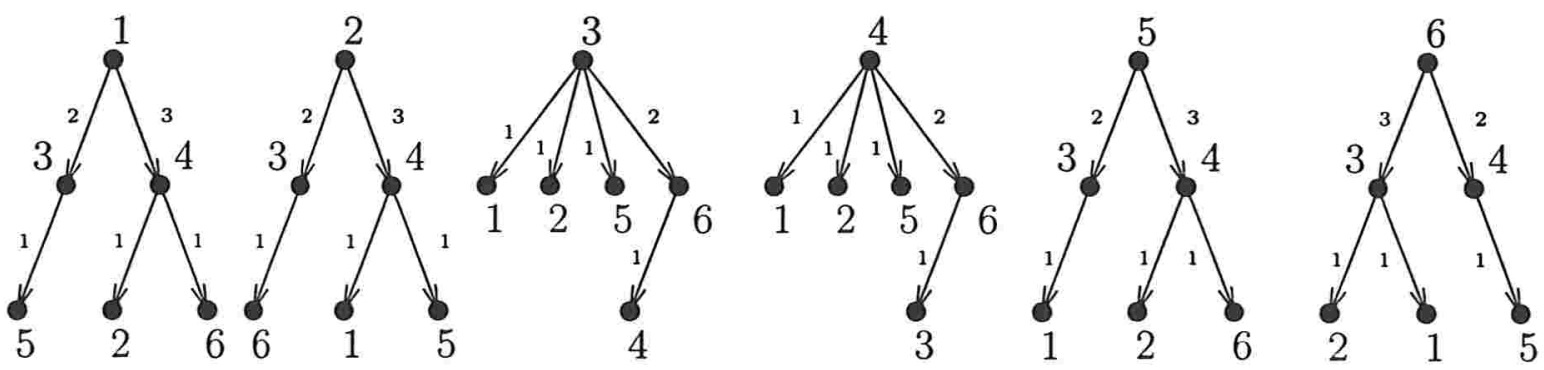
Proposição 3.29 (Vértices de $\mathcal{F}$ são fluxos coerentes) Se $f \in V(\mathcal{F})$, então qualquer decomposição $f=\sum_{r \in V} f^{r}$ onde $f^{r} \in F^{r}, \forall r$ é um fluxo coerente.

Prova : Análoga à de (3.24).

O mesmo exemplo apresentado anteriormente para o poliedro $\overrightarrow{\mathcal{F}}$ também serve para mostrar que o fato de $f \in \mathcal{F}$ ser um fluxo coerente não é condição suficiente para estabelecer que $f \in V(\mathcal{F})$.

Note que o resultado (3.29) permite-nos uma importante conclusão em relação ao (CFA):

Teorema 3.30 ((CFA) restrito aos fluxos coerentes) O problema (CFA) definido em (2.12) é equivalente a

$$
(C F A)\left\{\begin{array}{cc}
\min & D^{*}(f) \\
\text { s.a. } & f \in[F C]
\end{array}\right.
$$

onde $F C=\left\{f=\sum_{r \in V} f^{r} \in \mathcal{F} \mid f=\sum_{r \in V} f^{r}\right.$ é um fluxo coerente $\}$.

O resultado seguinte fornece duas caracterizações de vértices para $\mathcal{F}$, uma análoga a (1.31), e outra associada a um sistema alternativo (vide (1.27)):

Teorema 3.31 (Equivalências para $f \in V(\mathcal{F})$ ) Seja $f=\sum_{r \in V} f^{r} \in \mathcal{F}$ um fluxo coerente e denote por $\Pi_{0}^{i j}=\Pi_{f^{r}}^{i j}$. Denote ainda por $\Pi_{k}^{i j}, k=1, \ldots, k_{i j}$ todos os outros caminhos possiveis de $i$ a $j \mathrm{em} G$. As seguintes asserções são equivalentes:

1. f é vértice de $\mathcal{F}$.

2. $\exists a \in \mathbb{R}^{m}, a>0:\left\langle a, \Pi_{0}^{i j}\right\rangle\left\langle\left\langle a, \Pi_{k}^{i j}\right\rangle, \forall i, j, \forall k=1,2, \ldots, k_{i j}\right.$.

3. $\nexists u \geq 0:\left[\begin{array}{cccc} & \vdots & \vdots & \vdots \\ \Pi_{0}^{i j}-\Pi_{1}^{i j} \vdots \Pi_{0}^{i j}-\Pi_{2}^{i j \vdots} & \cdots & \vdots \\ \vdots & \vdots & \vdots & \vdots \\ \vdots & \vdots & \vdots\end{array}\right]\left[\begin{array}{c}u_{1}^{i j} \\ \vdots \\ u_{k_{i j}}^{i j} \\ \cdots \\ \forall i, j\end{array}\right] \geqq 0$.

Prova : Por conveniência, considere $\Pi^{i j} \in\{0,1\}^{m}$.

$(1 \Longleftrightarrow 2)$ Note que $f$ é vértice de $\mathcal{F} \Longleftrightarrow \exists c:\langle a, f\rangle\langle\langle a, x\rangle, \forall x \in \mathcal{F} /\{f\}$. Lembrando que $\mathcal{C}(\mathcal{F})=\mathbb{R}_{+}^{m}$, e tomando $x=f+d_{e}$, para cada $e \in E$, onde $\left\{d_{e}\right\}_{e \in E}$ representa a base canônica do $\mathbb{R}^{m}$, temos a seguinte conclusão:

$\exists a \in \mathbb{R}^{m}:\langle a, f\rangle<\langle a, x\rangle, \forall x \in \mathcal{F} /\{f\} \Longrightarrow \exists a \in \mathbb{R}^{m}, a>0:\langle a, f\rangle\langle\langle a, x\rangle, \forall x \in \mathcal{F} /\{f\}$. 
Para cada par $(i, j)$ e para cada $k=1, \ldots, k_{i j}$, seja $\varepsilon=q_{i j}>0$ e construa $x$ de tal forma que

$$
x_{e}= \begin{cases}f_{e}-\varepsilon & \forall e \in \Pi_{0}^{i j} / \Pi_{k}^{i j} \\ f_{e}+\varepsilon & \forall e \in \Pi_{k}^{i j} / \Pi_{0}^{i j} \\ f_{e} & \text { caso contrário. }\end{cases}
$$

Então $\langle a, f\rangle\left\langle\langle a, x\rangle\right.$ implica $\left\langle a, \Pi_{0}^{i j}\right\rangle\left\langle\left\langle a, \Pi_{k}^{i j}\right\rangle\right.$; fazendo isso para todos os pares $(i, j) \mathrm{e}$ $\forall k=1,2, \ldots, k_{i j}$ provamos que

$$
f \text { é vértice de } \mathcal{F} \Longrightarrow \exists a \in \mathbb{R}^{m}, a>0:\left\langle a, \Pi_{0}^{i j}\right\rangle\left\langle\left\langle a, \Pi_{k}^{i j}\right\rangle, \forall i, j, \forall k=1,2, \ldots, k_{i j} .\right.
$$

Isso prova $(1 \Longrightarrow 2)$. A volta é trivial pois se $x \neq f$ então pelo menos para um par $(i, j)$ o fluxo $x$ utiliza algum caminho diferente de $\Pi_{0}^{i j}$, de onde se conclui que $\langle a, f\rangle\langle\langle a, x\rangle$. Isto basta, por (1.31), para mostrar que $f \in V(\mathcal{F})$.

$(2 \Longleftrightarrow 3)$ Notando que o sistema (2) pode ser reescrito como

$$
\exists a>0:\left[\begin{array}{c}
\left(\Pi_{0}^{i j}-\Pi_{1}^{i j}\right)^{t} \\
\left(\Pi_{0}^{i j}-\Pi_{2}^{i j}\right)^{t} \\
\vdots \\
\left(\Pi_{0}^{i j}-\Pi_{k_{i j}}^{i j}\right)^{t} \\
\forall i j
\end{array}\right] a<0
$$

basta aplicar o teorema de alternativa de Gordan (1.27) e tem-se o resultado.

A condição 2 afirma que $f$ é vértice de $\mathcal{F}$ se, e somente se existe uma métrica, ou um conjunto de pesos positivos nas arestas para os quais os caminhos $\Pi_{0}^{i j}$ são todos estritamente mais curtos que quaisquer outros caminhos de $i$ a $j$.

Já a condição 3 admite a seguinte interpretação: cada coluna $\Pi_{k}^{i j}-\Pi_{0}^{i j}$ corresponde a um desvio de fluxo do caminho $\Pi_{0}^{i j}$ para o caminho $\Pi_{k}^{i j}$. Portanto para um fluxo coerente $f$ que é vértice não poderá haver uma seqüência não-vazia de desvios de fluxo de tal forma que o fluxo $f^{\prime}$ obtido pelos desvios de fluxo satisfaça $f^{\prime} \leq f$.

Um importante corolário de (3.31) é

Corolário 3.32 (Pré-imagem de $f \in V(\mathcal{F})$ ) Seja $\pi: \mathbb{R}^{2 m n} \longrightarrow \mathbb{R}^{m}$ a projeção tal que

$$
\mathcal{F}=\pi\left(\vec{F}^{1} \times \cdots \times \vec{F}^{n}\right) .
$$

Então dado $f=\sum_{r \in V} f^{r} \in V(\mathcal{F})$, a pré-imagem de $f$ é um conjunto unitário, dado por

$$
\pi^{-1}(f)=\left\{\left(f^{1}, \ldots, f^{n}\right)^{t}\right\}
$$

Em outros termos, a decomposição de $f$ em fluxos $f^{r s}$ tais que $f=\sum_{r \in V} \sum_{s \neq r} f^{r s}$ é única.

Prova : Imediata da demonstração de (2) $\Longrightarrow(1)$ em (3.31). 
Usando o teorema (3.31), vemos que em algumas situações especiais podemos garantir que um determinado fluxo coerente é um vértice de $\mathcal{F}$. Por exemplo:

Proposição 3.33 (Árvores são vértices de $\mathcal{F}$ ) Se $f=\sum_{r \in V} f^{r} \in \mathcal{F}$ é um fluxo coerente e $G_{f}$ é uma árvore, então $f \in V(\mathcal{F})$.

Prova : Esta afirmação pode ser demonstrada de várias formas. Verificaremos que $f$ satisfaz a condição 2 da proposição (3.31): considere o vetor $a$ dado por

$$
a_{e}= \begin{cases}1 & \text { se } f_{e}>0 \\ n & \text { caso contrário }\end{cases}
$$

Note que em qualquer caminho $\Pi_{k}^{i j}$ de $i$ a $j$ diferente de $\Pi_{0}^{i j}$ existe pelo menos um arco que não está na árvore $G_{f}$, e o custo neste arco, por contrução, é $n$. Como o caminho $\Pi_{0}^{i j}$ tem no máximo $n-1$ arcos, temos

$$
\left\langle a, \Pi_{k}^{i j}\right\rangle \geqq n>n-1 \geqq\left\langle a, \Pi_{0}^{i j}\right\rangle
$$

para todo caminho $\Pi_{k}^{i j}$ diferente de $\Pi_{0}^{i j}$, e para todo par $(i, j)$. Pela proposição (3.31), $f$ é um vértice de $\mathcal{F}$.

\subsubsection{Cômputo das arestas em $\mathcal{F}$}

Lembremos que o algorítmo de Tư̆-Zwart prevê que sejamos capazes de calcular o conjunto de arestas saindo de um vértice de $\mathcal{F}$. Conhecemos já uma caracterização necessária para estes vértices, o que nos permitiu concluir (3.30). Nesta seção, detalharemos um procedimento para o cômputo de um conjunto de direções viáveis saindo de um fluxo coerente $f$, e que no caso de $f \in V(\mathcal{F})$ coincide com as arestas saindo de $f$.

Com o auxílio do programa PORTA (Thomas Christof \& Andreas Löbel, disponível em ftp no endereço ftp://ftp.zib.de/pub/mathprog/polyth/porta/index.html) para análise de poliedros, pudemos constatar que qualquer caracterização de adjacências dos vértices de $\mathcal{F}$ não poderia basear-se somente nas árvores $f^{1}, \ldots, f^{n}$ associadas a estes. A estrutura de adjacências do poliedro $\mathcal{F}$ varia em função também das demandas $q_{r s}$. Para exemplificar, considere os fluxos coerentes dados pelas árvores
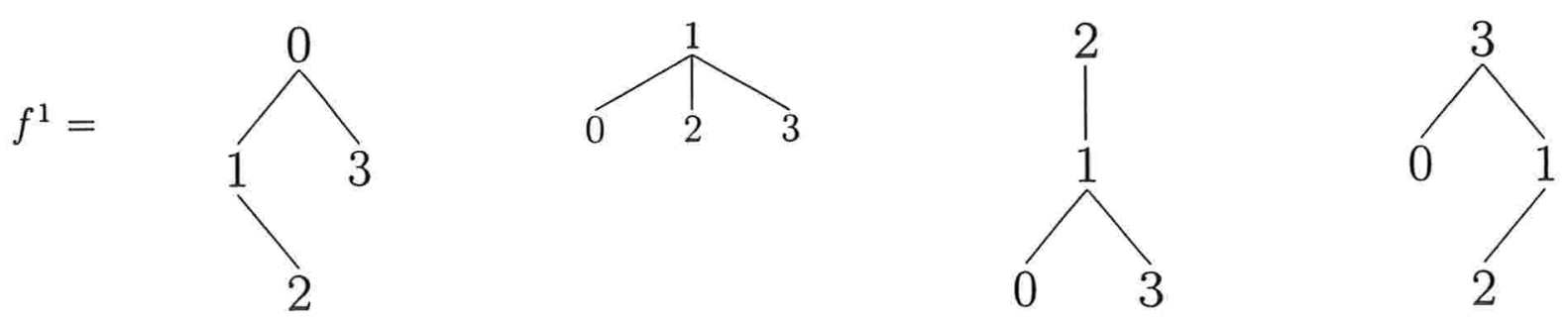

e 

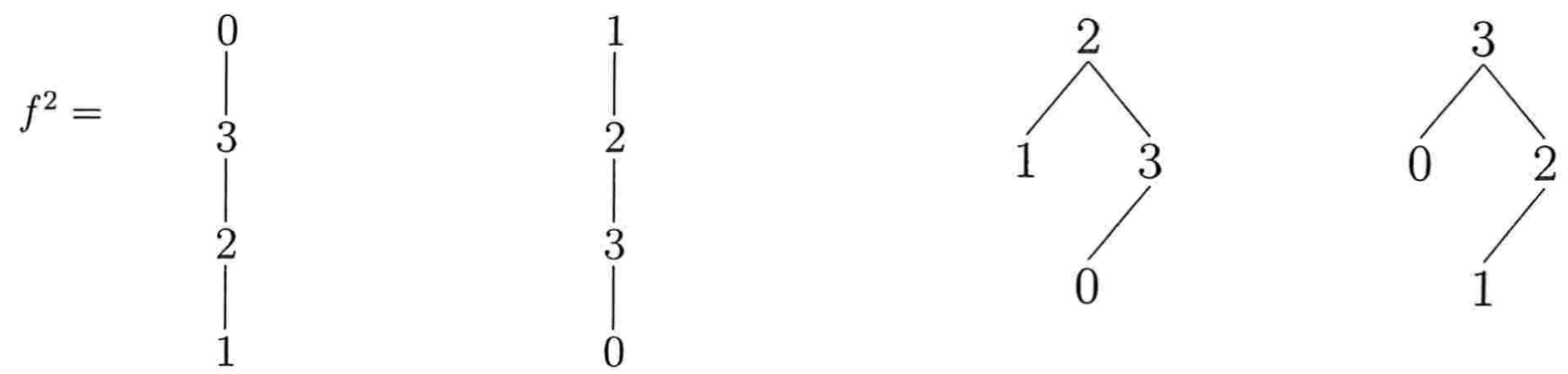

imersos no grafo

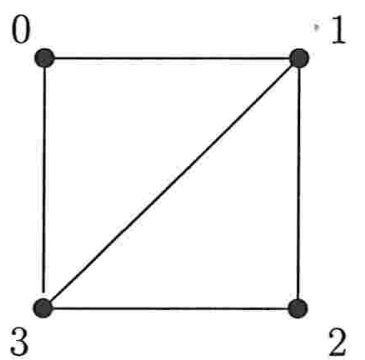

Estes fluxos são vértices do poliedro $\mathcal{F}$ de fluxos multicomodidade associado, e são adjacentes para $q_{01}=2, q_{r s}=1, \forall(r, s) \neq(0,1)$ (poliedro $P^{1}$ ), mas não são adjacentes para $q_{r s}=1, \forall r, s, r \neq s$ (poliedro $P^{2}$ ). Isto pode ser confirmado através das representações destes poliedros:

$P^{1}:\left\{\begin{array}{lllllll}x_{1} & & & +x_{4} & +x_{5} & \geqq 9 \\ & x_{2} & +x_{3} & +x_{4} & & \geqq 8 \\ x_{1} & +x_{2} & & & & \geqq 7 \\ & & x_{3} & & +x_{5} & \geqq 6 \\ x_{1} & & +x_{3} & +x_{4} & & \geqq 7 \\ & & & & +x_{4} & +x_{5} & \geqq 6 \\ & & & & x & \geqq 0\end{array}\right.$

$P^{2}:\left\{\begin{array}{lllllll}x_{1} & & & & +x_{4} & +x_{5} & \geqq 8 \\ & x_{2} & +x_{3} & +x_{4} & & \geqq 8 \\ x_{1} & +x_{2} & & & & \geqq 6 \\ & & x_{3} & & +x_{5} & \geqq 6 \\ x_{1} & & +x_{3} & +x_{4} & & \geqq 6 \\ & x_{2} & & +x_{4} & +x_{5} & \geqq & \\ & & & & x & \geqq & \\ & & & & & \end{array}\right.$

Nesta seção, desenvolvemos um método, que se mostrou eficiente, para o cálculo das direções de arestas saindo de um vértice $v \in \mathcal{F}$, a partir do método "inocente" de cômputo de arestas (algoritmo (1)).

Seja $f=\sum_{r \in V} f^{r} \in \mathcal{F}$ um fluxo qualquer, não necessariamente coerente, mas tal que $f^{r} \in V\left(F^{r}\right), \forall r \in V$. Vamos considerar todas as diferenças possíveis da forma $g-f$ onde $g=\sum_{r \in V} g^{r}$ é um fluxo diferente de $f$, também com a propriedade de que $g^{r} \in V\left(F^{r}\right), \forall r \in V$. Estas diferenças podem ser escritas como

$$
\begin{aligned}
g-f & =\sum_{r \in V} g^{r}-f^{r} \\
& =\sum_{r \in V} \sum_{s \neq r} g^{r s}-f^{r s},
\end{aligned}
$$

onde os termos $g^{r s}$ e $f^{r s}$ são $q_{r s^{-}}$-caminhos. Cada parcela $g^{r s}-f^{r s}$ é o que se chama um desvio de fluxo; vamos mostrar que estas parcelas são sempre combinações lineares positivas de desvios elementares e direções do cone: 
Definição 3.34 (Desvios elementares a partir de $f^{r} \in V\left(F^{r}\right)$ ) Seja $(i, j) \in \vec{E}$ um arco orientado, tal que $\{i, j\} \notin G_{f}^{r}$. Sejam $\Pi_{f}^{r i}$ e $\Pi_{f}^{r j}$ os caminhos válidos (em $G_{f}^{r}$ ) de $r$ a $i$ e de $r$ a $j$, respectivamente. Para simplificar a notação, assuma que $\Pi_{f}^{r i}, \Pi_{f}^{r j} \in\{0,1\}^{m}$. Definimos o desvio elementar em $G_{f}^{r}$ associado a $(i, j)$ como o vetor

$$
\Delta_{f r}^{i j}=\left[\begin{array}{c}
0 \\
\vdots \\
0 \\
1 \\
0 \\
\vdots \\
0
\end{array}\right]+\Pi_{f}^{r i}-\Pi_{f}^{r j},
$$

onde o vetor $w=(0, \ldots, 1, \ldots, 0)^{t}$ tem componente $w_{\{i, j\}}=1$.

Note que o vetor $\Delta_{f^{r}}^{i j}$ corresponde a uma variação do fluxo em $f^{r}$, transferindo parte do fluxo do caminho $\Pi_{f}^{r j}$ para o caminho $\Pi_{f}^{r i}$, acrescido do arco $(i, j)$.

Um desvio elementar a partir da $q_{r}$-árvore $f^{r}$ é obtido pela inclusão de exatamente um arco externo a esta $q_{r}$-árvore, como ilustrado a seguir:

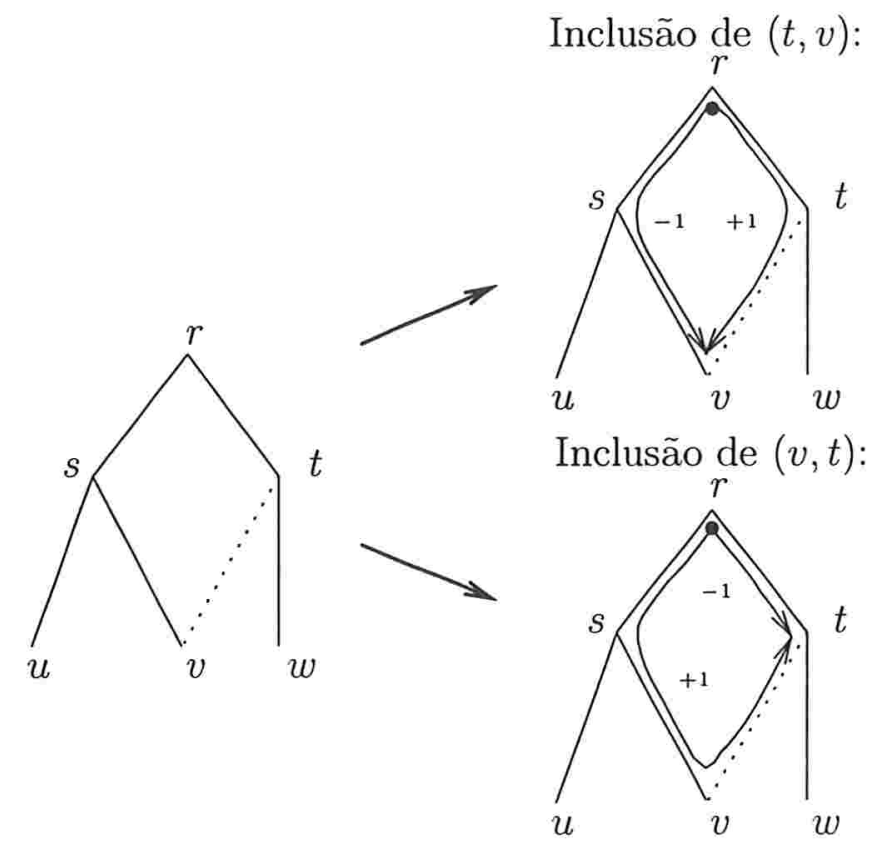

Desvios elementares associados ao arco $\{v, t\}$ 
Proposição 3.35 (Descrição da diferença $g^{r s}-f^{r s}$ ) Sejam $f^{r s}, g^{r s} \in V\left(F^{r s}\right)$. Podemos escrever

$$
g^{r s}-f^{r s}=\sum_{e \in \Pi_{g}^{r s}} \Delta^{e},
$$

onde $e=(i, j)$ são os arcos dirigidos associados $a \Pi_{g}^{r s}, e$

$$
\Delta^{e}=\left[\begin{array}{c}
0 \\
\vdots \\
0 \\
1 \\
0 \\
\vdots \\
0
\end{array}\right]+\Pi_{f}^{r i}-\Pi_{f}^{r j}
$$

como em (3.34).

Para um arco $e=\{i, j\} \notin G_{f}^{r}$, o vetor $\Delta^{e}$ corresponde ao desvio elementar em $G_{f}^{r}$ associado $a(i, j)$, como definido em (3.34). Se $\{i, j\}$ aparece em $G_{f}^{r}$, no sentido $(i, j)$, então $\Delta^{e}=0$. $E$ se $\{i, j\}$ aparece em $G_{f}^{r}$ no sentido $(j, i)$, então $\Delta^{e}$ é um raio extremal do cone $C(\mathcal{F})$.

Prova: Seja $\left(r=i_{0}, i_{1}, \ldots, i_{k}=s\right)$ o caminho $\Pi_{g}^{r s}$. Calculando $\sum_{e \in \Pi_{g}^{r s}} \Delta^{e}$ iremos obter a seguinte soma telescópica:

$$
\begin{aligned}
\sum_{e \in \Pi_{g}^{r s}} \Delta^{e} & =\sum_{j=0}^{k-1}\left[\begin{array}{c}
0 \\
\vdots \\
0 \\
1 \\
0 \\
\vdots \\
0
\end{array}\right]+\Pi_{f}^{\left.r i_{j}, i_{j+1}\right\}}-\Pi_{f}^{r i_{j+1}} \\
& =\Pi_{f}^{r i_{0}}-\Pi_{f}^{r i_{k}}+\sum_{j=0}^{k-1}\left[\begin{array}{c}
0 \\
\vdots \\
0 \\
1< \\
0 \\
\vdots \\
0
\end{array}\right]{ }^{\left\{i_{j}, i_{j+1}\right\}} \\
& =\Pi_{f}^{r r}-\Pi_{f}^{r s}+\Pi_{g}^{r s} \\
& =g^{r s}-f^{r s} .
\end{aligned}
$$

Note que o vetor $\Delta^{e}$ definido no enunciado pode ser visto informalmente como o desvio de fluxo da figura a seguir: 


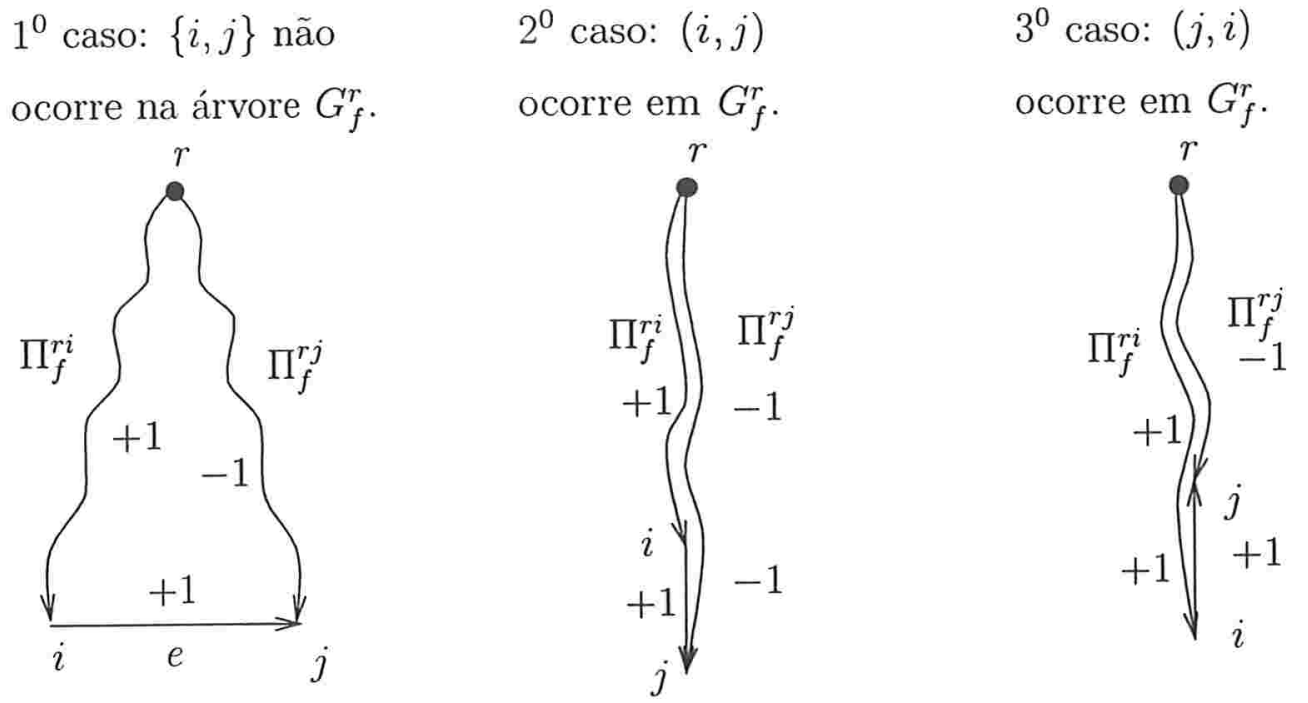

Desvio $\Delta^{e}$ associado ao arco $e=(i, j)$ do caminho $\Pi_{g}^{r s}$

É fácil ver que, no caso de $(i, j)$ ocorrer na $q_{r}$-árvore $G_{f}^{r}$, então o caminho $\Pi_{f}^{r j}$ será exatamente o caminho $\Pi_{f}^{r i}$ acrescido do arco $(i, j)$, e neste caso

$$
\left.\Delta^{e}=\left[\begin{array}{c}
0 \\
\vdots \\
0 \\
1 \\
0 \\
\vdots \\
0
\end{array}\right]+\Pi_{f}^{r i}-\Pi_{f}^{r j}=\left[\begin{array}{c}
0 \\
\vdots \\
0 \\
1 \\
0 \\
\vdots \\
0
\end{array}\right]\left\{\begin{array}{c}
\{i, j\} \\
\vdots \\
0 \\
1 \\
0 \\
\vdots \\
0
\end{array}\right]\right\}^{\{i, j\}}=0
$$

No caso de $(j, i)$ ocorrer em $G_{f}^{r}$, então $\Pi_{f}^{r i}$ será exatamente o caminho $\Pi_{f}^{r j}$ acrescido do $\operatorname{arco}(j, i)$, e conseqüentemente,

$$
\Delta^{e}=\left[\begin{array}{c}
0 \\
\vdots \\
0 \\
1 \\
0 \\
\vdots \\
0
\end{array}\right]+\prod_{f}^{r i}-\Pi_{f}^{r j}=\left[\begin{array}{c}
0 \\
\vdots \\
0 \\
1 \\
0 \\
\vdots \\
0
\end{array}\right]+\left[\begin{array}{c}
\{i, j\} \\
\vdots \\
0 \\
1 \\
0 \\
\vdots \\
0
\end{array}\right]=\left[\begin{array}{c}
0 \\
\{i, j\} \\
\vdots \\
0 \\
2 \\
0 \\
\vdots \\
0
\end{array}\right] \underbrace{\{i, j\}}
$$

Isto conclui a prova.

A partir desta proposição, sabemos que para quaisquer fluxos $f$ e $g$, tais que $f^{r}, g^{r} \in V\left(F^{r}\right)$, $\forall r \in V$, temos que $g-f=\sum_{i=1}^{L} \Delta^{e}+h$, onde $h \in C(\mathcal{F})$ e $\Delta^{e}$ são desvios elementares nas diversas 
árvores $f^{1}, \ldots, f^{n}$. O número máximo de desvios elementares em cada uma das árvores $f^{r}$ é dado pelo número de arcos que não estão em $f^{r}$, nos dois sentidos; portanto para cada árvore $f^{r}$ teremos no máximo $2(m-(n-1))=2 m-2 n+2$ desvios elementares; Conseqüentemente, o total de desvios elementares que podem ser gerados a partir de $f$ é, no máximo, $2 m n-2 n^{2}+2 n$.

Corolário 3.36 (Aproximação cônica a partir de $f \in \mathcal{F}$ ) Seja $f \quad \in \quad \mathcal{F}$, tal que $f^{r} \in V\left(F^{r}\right), \forall r \in V$, e sejam $\left\{\Delta^{k}\right\}_{k=1}^{L}$ todos os desvios elementares gerados pela inclusão de algum arco $(i, j)$ em uma das $q_{r}$-árvores $G_{f}^{r}$; sejam $\left\{h^{j}\right\}_{j=1}^{m}$ os raios extremais de $C(\mathcal{F})$. Então o conjunto

$$
D=\left\{\Delta^{k}\right\}_{k=1}^{L} \bigcup\left\{h^{j}\right\}_{j=1}^{m}
$$

fornece uma aproximação cônica do poliedro $\mathcal{F}$ a partir de $f$; isto é, para todo ponto $x \in \mathcal{F}$, existem $\beta \geqq 0$ e $\eta \geqq 0$ tais que

$$
x=f+\sum_{k=1}^{L} \beta_{k} \Delta^{k}+\sum_{j=1}^{m} \eta_{j} h^{j} .
$$

Prova: Se $x \in \mathcal{F}=[V(\mathcal{F})]+C(\mathcal{F})$, então existem $h \in C(\mathcal{F})$ e $\lambda \geqq 0$ tais que $\sum_{i=1}^{p} \lambda_{i}=1$ e $x=\sum_{i=1}^{p} \lambda_{i} v^{i}+h$, onde $\left\{v^{i}\right\}_{i=1}^{p}$ são vértices de $\mathcal{F}$. Logo $x=f+\sum_{i=1}^{p} \lambda_{i}\left(v^{i}-f\right)+h$.

Por (3.22) sabemos que cada $v^{i}$ é tal que $\left(v^{i}\right)^{r} \in V\left(F^{r}\right), \forall r \in V$, e por (3.35) sabemos que as diferenças $\left(v^{i}-f\right)$ podem ser escritas como combinações lineares positivas de desvios elementares gerados a partir de $f$. Além disso, $h=\sum_{j=1}^{m} \eta_{j} h^{j}$. Logo

$$
x=f+\sum_{k=1}^{L} \beta_{k} \Delta^{k}+\sum_{j=1}^{m} \eta_{j} h^{j}
$$

onde $\beta \geqq 0, \eta \geqq 0$ e $\Delta^{k}$ são desvios elementares gerados a partir das árvores $f^{1}, \ldots, f^{n}$.

Note que até agora a única hipótese sobre $f=\sum_{r \in V} f^{r}$ é que cada $f^{r}$ seja um vértice de $F^{r}$; $f$ não precisa ser vértice de $\mathcal{F}$, e na verdade $f$ não precisa nem ser um fluxo coerente.

Podemos eliminar as direções positivamente linearmente dependentes descritas em (3.36), até formarmos um conjunto de direções positivamente linearmente independentes e que fornecem uma aproximação cônica de $\mathcal{F}$ a partir de $f$. Se $f$ é um vértice, então o conjunto gerado é o das direções de arestas saindo de $f$, por (1.33). 
Algorítmo 5 (Cômputo de arestas saindo de $f \in V(\mathcal{F})$ )

1. Inicialize o conjunto $X$ com todas as direções dadas por desvios elementares a partir de $f$, bem como todos os $m$ raios extremais do cone. Denote $X=\left\{d^{1}, \ldots, d^{k}\right\}$.

2. Para cada direção di verifique se o sistema

$$
d^{i}=\sum_{j \neq i} \beta_{j} d^{j}, \beta \geqq 0
$$

admite alguma solução; Em caso positivo, faça $X \longleftarrow X \backslash\left\{d^{i}\right\}$.

Este método devolve em $X$ o conjunto de todas as direções de arestas saindo de $f$.

No passo 2 pode ser utilizado a fase 0 do simplex. No caso do simplex tabular, podem ser eliminadas várias direções positivamente linearmente dependentes em apenas uma execução da fase 0; basta observar que, no tableau ótimo, todas as variáveis não-básicas que indicam ilimitação (isto é, para as quais $A_{B}^{-1} A^{j} \geqq 0$ ) estão associadas a direções $d^{j}$ que também se escrevem como combinação linear positiva das direções associadas às variáveis básicas.

Note que o número de direções geradas na inicialização de (5) pode ser reduzido utilizando o fato de $f$ ser um fluxo coerente, e o conceito de raiz de um desvio elementar:

Definição 3.37 (Raiz de um desvio elementar) Seja $f^{r} \in V\left(F^{r}\right)$ e $\Delta$ o desvio elementar em $G_{f}^{r}$ associado a $(i, j)$. Definimos a raiz do ciclo elementar $\Delta$, denotada por $r_{\Delta}$, como o último nó comum aos caminhos $\Pi_{f}^{r i} e \Pi_{f}^{r j}$.

Note que se os caminhos $\Pi_{f}^{r i}$ e $\Pi_{f}^{r j}$ são disjuntos nas arestas, então $r_{\Delta}=r$.

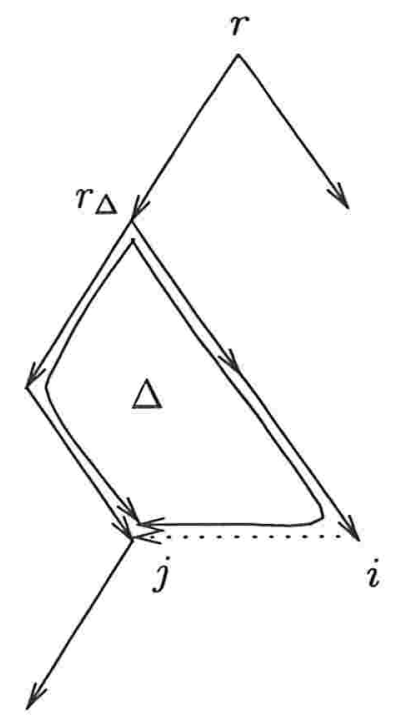

\section{Raiz do desvio elementar $\Delta$}


Proposição 3.38 (Inicialização do algorítmo (5)) Seja $f=\sum_{r \in V} f^{r} \in \mathcal{F}$ um fluxo coerente. No passo 1 do algorítmo (5), podemos inicializar o conjunto $X$ apenas com os desvios elementares em $G_{f}^{r}$ com raiz $r_{\Delta}=r$.

Prova : Suponha que na introdução do arco $(i, j)$ na árvore $G_{f}^{r}$ o desvio elementar $\Delta$ gerado não inclua a raiz $r$. Neste caso poderemos identificar uma raiz $r_{c}$ do ciclo, isto é, o primeiro nó comum aos caminhos $\Pi_{f}^{r i}$ e $\Pi_{f}^{r j}$; pela coerência sabemos que o mesmo ciclo será identificado na árvore de raiz $r_{\Delta}$, ou seja, na árvore $G_{f}^{r_{\Delta}}$ pela inclusão do mesmo arco $(i, j)$. Isto prova o enunciado.

Note que o algorítmo (5) nos permite enunciar:

Proposição 3.39 (Direções de arestas no poliedro $\mathcal{F}$ ) Todas as direções de arestas em $\mathcal{F}$ correspondem ou a desvios elementares ou a raios extremais do cone $C(\mathcal{F})$. Todas têm a forma geral

$$
\left[\begin{array}{c}
0 \\
\vdots \\
+\Delta \\
\vdots \\
-\Delta \\
\vdots \\
0
\end{array}\right] \text { ou }\left[\begin{array}{c}
0 \\
\vdots \\
0 \\
1 \\
0 \\
\vdots \\
0
\end{array}\right]
$$

\subsubsection{Descrição de $\mathcal{F}$ por Cortes}

Nesta seção fornecemos uma descrição parcial do conjunto das facetas de $\mathcal{F}$, dadas por desigualdades associadas a cortes minimais do grafo $G$, análogas às das proposições (3.8) e (3.17). A descrição dada pelos cortes não é completa, como veremos no exemplo do final desta seção.

Proposição 3.40 (Representação parcial de $\mathcal{F}$ por cortes) Sejam $S_{1}, S_{2}, \ldots, S_{k}$ todos os subconjuntos de $V$ tais que $\emptyset \neq S_{i} \neq V$ e $\delta\left(S_{i}\right)$ é um corte minimal no grafo $G$. Então

$$
\mathcal{F} \subset\left\{x \in \mathbb{R}_{+}^{m} \mid \sum_{e \in \delta\left(S_{i}\right)} x_{e} \geqq \sum_{r \in S_{i},} \sum_{s \in V \backslash S_{i}} q_{r s}+q_{s r}, i=1, \ldots, k\right\} ;
$$

Mais ainda, todas as desigualdades acima definem facetas do poliedro $\mathcal{F}$.

Prova : Seja $x \in \mathcal{F}$, e sejam $f^{r} \in \vec{F}^{r}$, para $r \in V$ tais que $x$ é a projeção de $\sum_{r \in V} f^{r}$. Então para cada $f^{r}$ e cada $i$ temos duas possibilidades. Se $r \in S_{i}$, então somando as equações de (3.9) para $j \in S_{i}$, vemos que $f^{r}$ satisfaz:

$$
\sum_{e \in \delta^{+}\left(S_{i}\right)} f_{e}^{r}-\sum_{e \in \delta^{-}\left(S_{i}\right)} f_{e}^{r}=\sum_{s \in V \backslash S_{i}} q_{r s}
$$


se por outro lado $r \in V \backslash S_{i}$ então $f^{r}$ satisfaz:

$$
\sum_{e \in \delta^{+}\left(V \backslash S_{i}\right)} f_{e}^{r}-\sum_{e \in \delta^{-}\left(V \backslash S_{i}\right)} f_{e}^{r}=\sum_{s \in S_{i}} q_{r s}
$$

Então, para cada $i=1, \ldots, k$ :

$$
\begin{aligned}
\sum_{e \in \delta\left(S_{i}\right)} x_{e}= & \sum_{r \in V} \sum_{e \in \delta^{+}\left(S_{i}\right)} f_{e}^{r}+\sum_{e \in \delta^{-}\left(S_{i}\right)} f_{e}^{r} \\
\geqq & \sum_{r \in S_{i}}\left(\sum_{e \in \delta^{+}\left(S_{i}\right)} f_{e}^{r}-\sum_{e \in \delta^{-}\left(S_{i}\right)} f_{e}^{r}\right) \\
& +\sum_{r \in V \backslash S_{i}}\left(\sum_{e \in \delta^{+}\left(V \backslash S_{i}\right)} f_{e}^{r}-\sum_{e \in \delta^{-}\left(V \backslash S_{i}\right)} f_{e}^{r}\right) \\
= & \sum_{r \in S_{i}} \sum_{s \in V \backslash S_{i}} q_{r s}+\sum_{r \in V \backslash S_{i}} \sum_{s \in S_{i}} q_{r s} \\
= & \sum_{r \in S_{i}} \sum_{s \in V \backslash S_{i}} q_{r s}+q_{s r} .
\end{aligned}
$$

Para concluir que cada uma destas desigualdades é uma faceta, como em (3.17), mostraremos que para cada $i$ existem $m$ pontos do poliedro $x^{0}, \ldots, x^{m}$ que satisfazem a restrição associada a $S_{i}$ com igualdade e tais que $\left\{x^{1}-x^{0}, \ldots, x^{m}-x^{0}\right\}$ é L.I.

Tome um $i$ qualquer, e seja $\delta\left(S_{i}\right)=\left\{e_{0}, \ldots, e_{k}\right\}$. Para cada uma das arestas $e_{j}$, escolha uma árvore qualquer que passe em $e_{j}$ mas não passe nas outras arestas do corte (se tal árvore não existisse, o corte $S_{i}$ não seria minimal); chame de $x^{j}$ o fluxo multicomodidade correspondente a enviar todas as mensagens pelos arcos desta árvore. Até agora temos $k$ pontos que satisfazem a restrição de $S_{i}$ com igualdade. Para cada aresta $e_{j} \notin \delta\left(S_{i}\right)$, podemos construir os pontos da forma $x^{j}=x^{0}+M e_{j}$, escolhendo um $M>0$ suficientemente grande de forma que $\left\{x^{0}, \ldots, x^{m}\right\}$ seja L.I. Cada um destes pontos satisfaz a restrição associada a $S_{i}$ com igualdade, como pode ser facilmente verificado, completando a prova.

Novamente com o auxílio do programa PORTA, pudemos analisar as facetas de alguns poliedros $\mathcal{F}$ para grafos de até 8 nós. Através de uma enumeração explícita de todos os fluxos coerentes e conhecendo a priori os raios extremais de $C(\mathcal{F})$, fornecemos ao programa uma descrição do poliedro da forma $[U]+C$, que é transformada então em uma representação minimal da forma $A x \leq b$, onde cada desigualdade define uma faceta (como o poliedro tem dimensão $m$, qualquer representação minimal só possui facetas (1.36)).

O exemplo seguinte mostra que uma faceta de $\mathcal{F}$ pode estar associada a um corte que não é minimal. Sendo $G$ o grafo

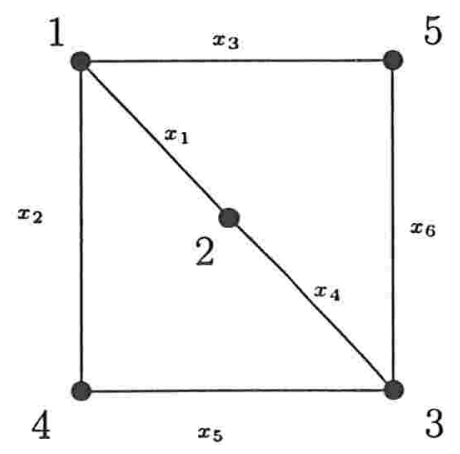


e $q_{r s}=1, \forall r, s, r \neq s$, obtivemos a seguinte descrição das facetas:

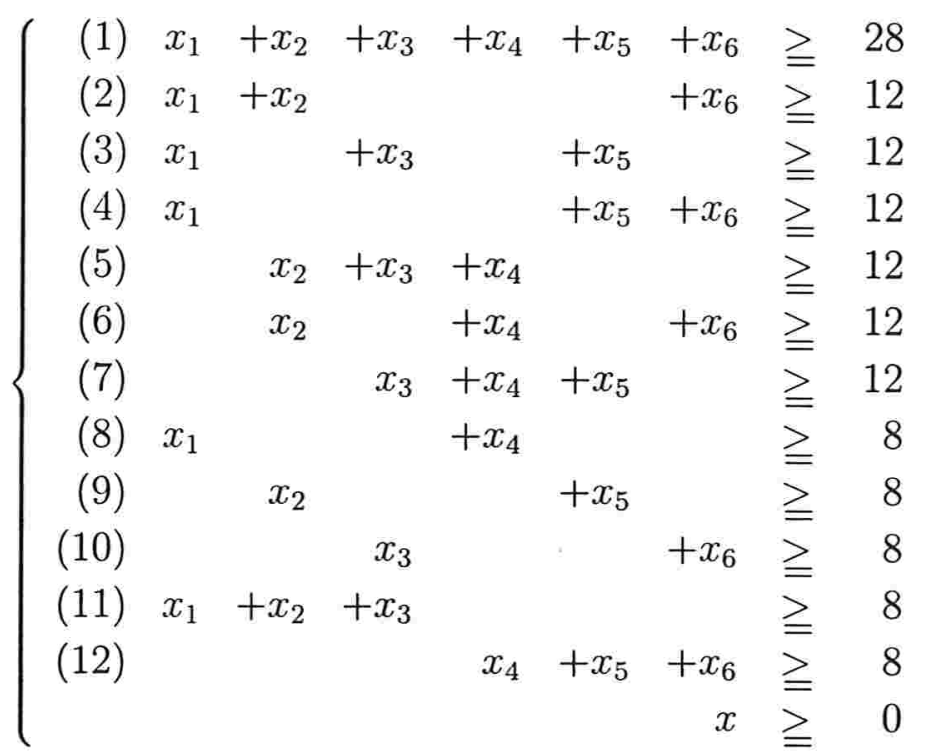

Ilustramos a seguir alguns os cortes associados a algumas destas facetas. Note que o corte associado à faceta (1) não é minimal:

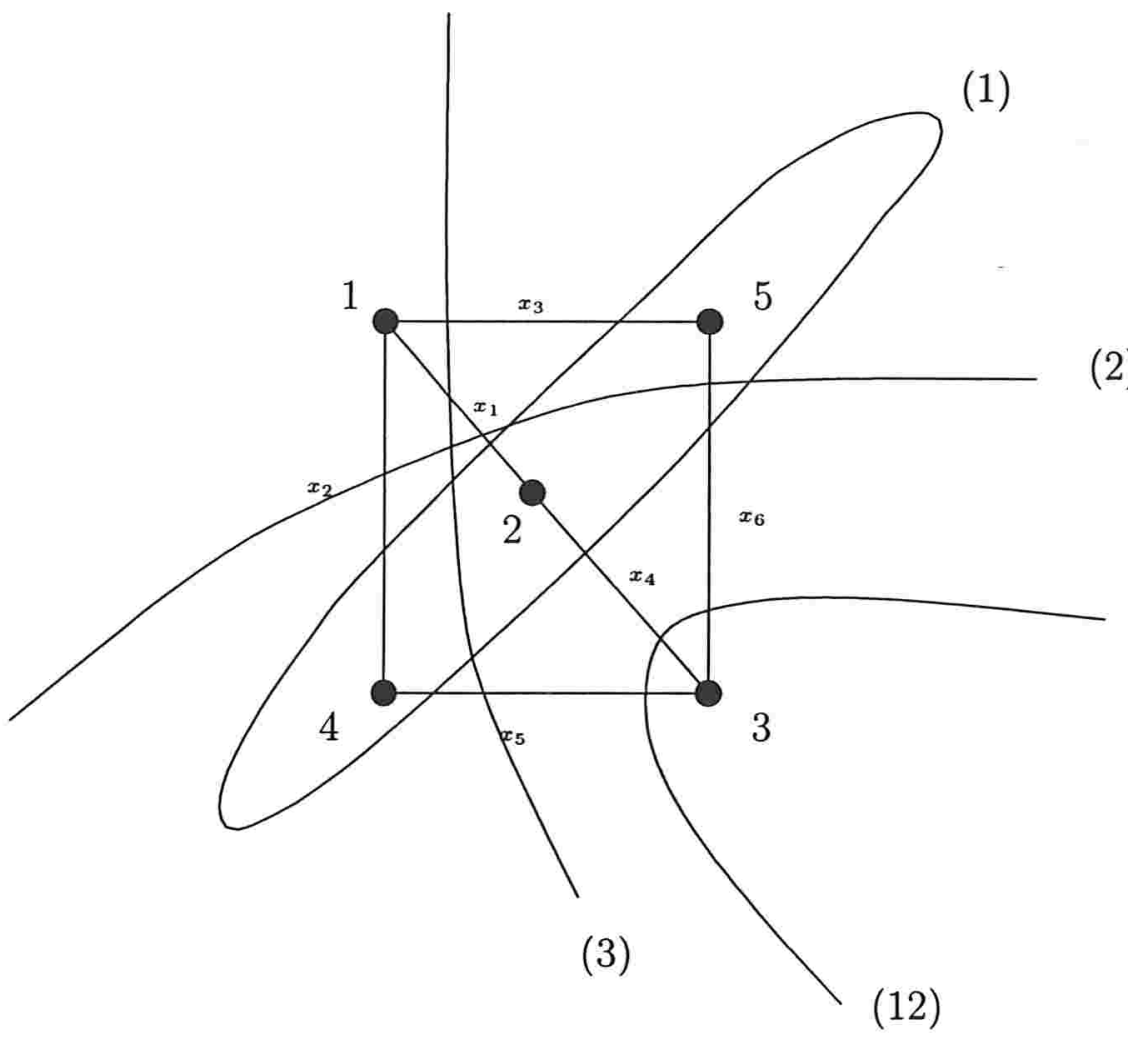

Nem mesmo é verdade que todas as facetas correspodem a cortes no grafo $G$. Sendo $G$ o grafo 


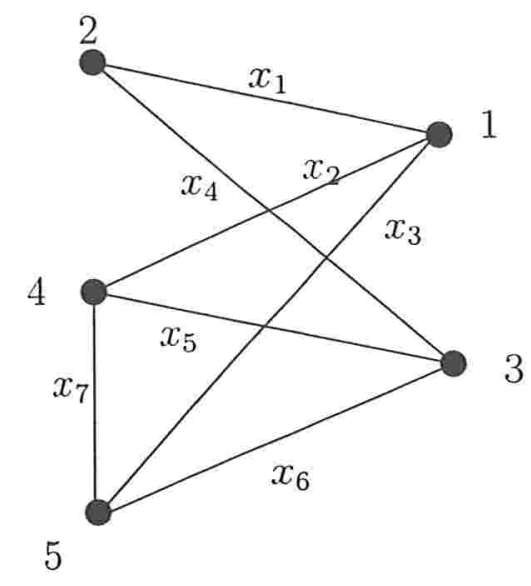

e $q_{r s}=1, \forall r, s, r \neq s$, a primeira desigualdade do sistema:

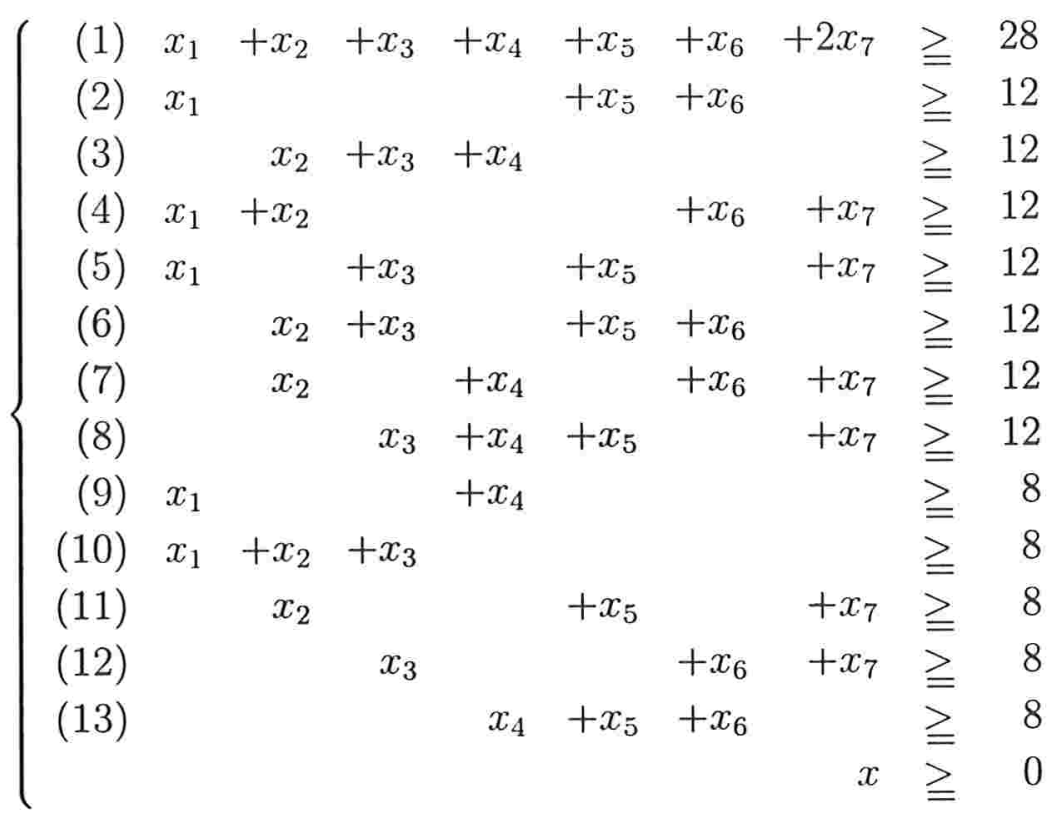

não corresponde a um corte (o coeficiente de $x_{7}$ é 2).

No entanto o poliedro de cortes definido em (3.40) certamente contém $\mathcal{F}$, e além disso é descrito por facetas de $\mathcal{F}$, o que poderia ser aproveitado em alguma estratégia do tipo FalkHoffman (algorítmo (4)); veja o capítulo (5). 


\section{Capítulo 4}

\section{Algorítmos e Heurísticas}

Neste capítulo apresentaremos duas abordagens heurísticas para a solução do (CFA) utilizando a estrutura do poliedro $\mathcal{F}$ (seção (3.3)), bem como o algoritmo de Tŭ̌-Zwart (3) aplicado ao poliedro $\vec{F}^{1} \times \vec{F}^{2} \times \cdots \times \vec{F}^{n}$, enfatizando as diferenças de aplicação em relação à formulação dada em (3). Em seguida discutiremos alguns resultados de testes computacionais efetuados, comparando as heurísticas e apontando algumas dificuldades práticas de implementação do algoritmo descrito na seção (4.2). Todos os códigos referentes às heurísticas e ao algorítmo podem ser obtidos por ftp anônimo em ftp://ftp.ime.usp.br/pub/mqz/

\subsection{Heurísticas Baseadas em Tŭ-Zwart}

A idéia básica das heurísticas apresentadas nesta seção, é utilizar o conjunto finito de fluxos coerentes. Pela proposição (3.29) sabemos que qualquer vértice de $\mathcal{F}$ será um fluxo coerente, e restringimos portanto o (CFA) à formulação dada pelo teorema (3.30):

$$
(C F A)\left\{\begin{array}{cc}
\min & D^{*}(f) \\
\text { s.a. } & f \in[F C]
\end{array}\right.
$$

onde $F C=\left\{f=\sum_{r \in V} f^{r} \in \mathcal{F} \mid f=\sum_{r \in V} f^{r}\right.$ é um fluxo coerente $\}$.

Note que a partir de um fluxo coerente $f$, temos um método para o cômputo de direções que saem deste fluxo, e fornecem uma aproximação cônica do poliedro a partir de $f$ (algoritmo (5)). Se $f$ é um vértice, este método calcula o conjunto de todas as arestas saindo de $f$.

Os novos vértices introduzidos pelos cortes de Tư̆ não possuem em geral uma caracterização tão simples quanto a dos fluxos coerentes. Uma abordagem heurística possível é simplesmente ignorar a existência destes vértices novos, já que sempre existirá um fluxo coerente que é minimo global para o (CFA) (proposição (1.21)). Introduzimos os cortes de Tŭ̌, e exploramos apenas fluxos coerentes que obedeçam aos cortes já introduzidos. Esta é, em linhas gerais a descrição da heurística (1) para o (CFA). Antes de apresentar a heurística, um resultado fundamental:

Proposição 4.1 (Mínimos locais são vértices) Se $f=\sum_{r \in V} f^{r} \in \mathcal{F}$ é um fluxo, com $f^{r} \in V\left(F^{r}\right)$ para cada $r \in V$, e $f$ é mínimo local em relação às direções geradas em (5), então $f \in V(\mathcal{F})$. 
Prova : Lembremos que as $p$ direções $d^{k}$ geradas em (5) fornecem uma aproximação cônica de $\mathcal{F}$ a partir de $f$.

Suponha por contradição que $f \notin V(\mathcal{F})$. Então podemos escrever, para as direções $d^{k}$

$$
\sum_{k=1}^{p} \beta_{k} d^{k}=0
$$

onde $\beta \geq 0$. Mas $f$ é mínimo local em relação às direções $d^{k}$, ou seja, $D^{*}(f)<D^{*}\left(x^{k}\right)$ onde $x^{k}=f+\theta^{k} d^{k}$ como construídos em (2.21); disto concluímos que

$$
D^{*}(f) \leqq \min \left\{D^{*}\left(x^{k}\right) \mid k=1, \ldots, p\right\}
$$

Porém pela expressão $\sum_{k=1}^{p} \beta_{k} d^{k}=0, \operatorname{com} \beta \geq 0$, e pela quasiconcavidade estrita da função $D^{*}(\cdot)$ (2.18), podemos escrever

$$
D^{*}(f)>\min \left\{D^{*}\left(x^{k}\right) \mid k=1, \ldots, p\right\},
$$

já que $f$ pode ser escrito como $f \in\left[\left\{x^{k}\right\}_{k=1}^{p}\right]$, pela construção dos pontos $x^{k}$. Isto conclui a prova.

Heurística 1 (Heurística (1) para o (CFA)) Seja $\varepsilon>0$ fixado.

1. Encontre um fluxo coerente inicial $f^{0}$. Faça Valor Ótimo $=D^{*}\left(f^{0}\right)$, Ótimo $=f^{0}$, Restrições $=\emptyset$ e $i=0$

2. Encontre um mínimo local z a partir de $f^{i}$. Se isto não for possível, pare! Caso contrário, este minimo local é um vértice de $\mathcal{F}$ (proposição (4.1)). Se o valor de $D^{*}(z)$ é menor do que Valor Ótimo, atualize Valor Ótimo e Ótimo.

3. Gere as direçôes saindo de z (através do algoritmo (5)), e calcule o corte de Tŭ gerado por $z$ (2.23). Seja $\langle a, x\rangle \geqq b$ este corte.

4. Maximize a função $\langle a, x\rangle$, percorrendo somente fluxos que respeitem todas as restrições do conjunto Restrições.

5. Se a solução ótima $x^{*}$ do passo 4 tem valor $\left\langle a, x^{*}\right\rangle>b+\varepsilon$, então faça Restrições $\longrightarrow$ Restrições $\cup\{\langle a, x\rangle \geqq b\}, f^{i+1}=x^{*}, i \longrightarrow i+1$, e volte para o passo 2. Caso contrário, pare!

Agora explicaremos em detalhe cada um dos passos desta heurística.

O primeiro passo consiste em encontrar um fluxo coerente inicial. Isso pode ser feito encontrando-se uma árvore geradora do grafo $G$, e enviando todos os fluxos através dos caminhos desta árvore. Este fluxo, além de coerente, corresponde a um vértice de $\mathcal{F}$, por (3.33). 
No segundo passo, encontrar um mínimo local a partir de $f^{i}$ significa percorrer um conjunto de fluxos coerentes através das direções construídas em (5), até encontrar um fluxo coerente, a partir do qual todas as direções construídas levam a fluxos com valor de $D^{*}(\cdot)$ superior a Valor ótimo, ou são direções de ilimitação de $\mathcal{F}$ (neste caso, direções da base canônica do $\mathbb{R}^{m}$ ). Voltaremos a abordar em detalhe o problema de andar a partir de um fluxo coererente em uma direção dada pelo algorítmo (5).

As buscas uni-direcionais para determinar o plano de corte de Tul̆, seriam normalmente feitas com técnicas utilizando informações da derivada de $D^{*}(\cdot)$, mas por causa do efeito "calha" mencionado na seção (2.3), optamos por uma estratégia de busca binária aliada ao método da falsa posição:

Algorítmo 6 (Buscas unidirecionais) Seja $f=\sum_{r \in V} f^{r} \in V(\mathcal{F})$ um mínimo local, e $d^{k}$ uma direção gerada pelo algorítmo (5). Seja $\varepsilon>0$, e considere todas as relações $(\geqq, \leqq e=)$ a seguir aproximadas, com precisão dada por $\varepsilon$.

1. Se $d^{k} \in C(\mathcal{F})$, então $D^{*}(\cdot)$ é crescente na semi-reta $\left\{f+\theta d^{k} \mid \theta \geqq 0\right\}$. Devolva $\theta_{k}=\infty$.

2. Se $d^{k} \notin C(\mathcal{F})$, determine

$$
\theta_{k}=\arg \max \left\{\theta \mid \theta \geqq 0 \text { e } D^{*}\left(f+\theta d^{k}\right) \geqq D^{*}(f)\right\} .
$$

Faça $x^{k}=f+\theta_{k} d^{k}$.

3. Se $D^{*}\left(x^{k}\right) \geqq$ Valor Ótimo, devolva $\theta_{k}$.

4. Caso contrário, $D^{*}\left(x^{k}\right)<$ Valor Ótimo. Por busca binária, encontraremos $\alpha$ e $\beta$ tais que $D^{*}\left(f+\alpha d^{k}\right)>$ Valor Ótimo e $D^{*}\left(f+\beta d^{k}\right)<$ Valor Ótimo. Faça $\alpha=0$ e $\beta=\theta_{k}$.

5. Calcule $\eta=\frac{\alpha+\beta}{2}$. Se $D^{*}\left(f+\eta d^{k}\right)<$ Valor Ótimo, faça $\beta=\eta$ e volte para o passo 5 . Se $D^{*}\left(f+\eta d^{k}\right)=$ Valor Ótimo, devolva $\theta_{k}=\eta$. Se $D^{*}\left(f+\eta d^{k}\right)>$ Valor ótimo, faça $\alpha=\eta$.

6. Temos agora $D^{*}\left(f+\alpha d^{k}\right)>$ Valor Ótimo e $D^{*}\left(f+\beta d^{k}\right)<$ Valor ótimo. Pelo método da falsa posição, encontraremos $\eta$ tal que $D^{*}\left(f+\eta d^{k}\right)=$ Valor ótimo.

7. Calcule

$$
\eta=\alpha+\left(D^{*}\left(f+\alpha d^{k}\right)-\text { Valor Ótimo }\right) \frac{\beta-\alpha}{D^{*}\left(f+\alpha d^{k}\right)-D^{*}\left(f+\beta d^{k}\right)}
$$

8. Se $D^{*}\left(f+\eta d^{k}\right)>$ Valor Ótimo, faça $\alpha=\eta$ e volte ao passo $\%$.

9. Devolva $\theta_{k}=\eta$.

As técnicas de busca binária e da falsa posição são ilustradas a seguir. 

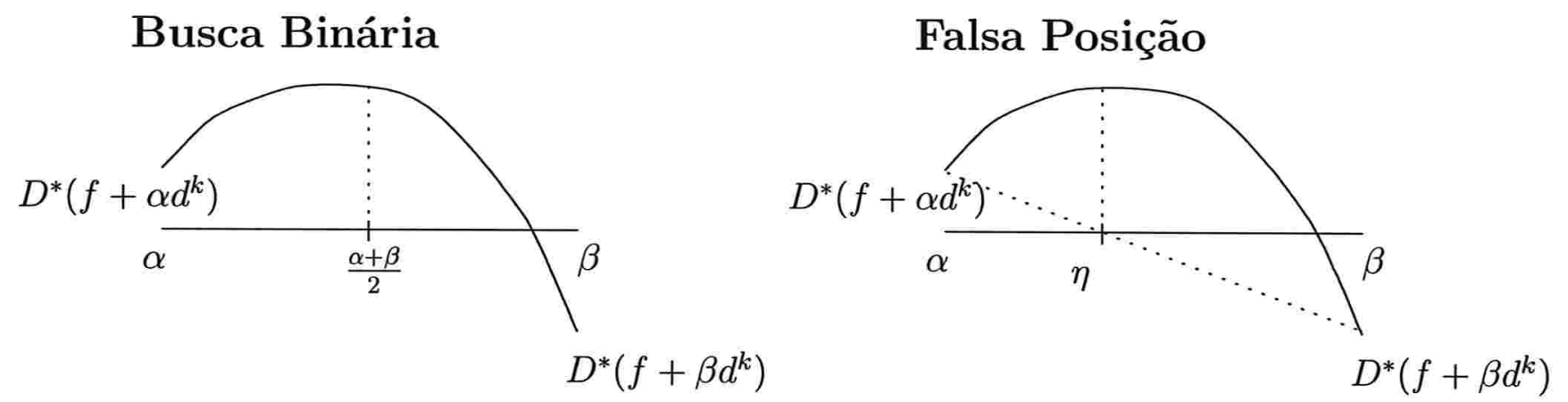

Lembremos que se a direção $d^{k}$ é dada por um raio do cone, então $D^{*}(\cdot)$ é estritamente crescente nesta direção. Isto significa que não precisaremos fazer buscas uni-direcionais, bem como procurar fluxos coerentes, nestas direções:

\section{Proposição 4.2 (Coeficiente do corte de Tŭ para $d^{k} \in C(\mathcal{F})$ )}

Seja $f=\sum_{r \in V} f^{r} \in V(\mathcal{F})$ um mínimo local, e $d^{k} \in C(\mathcal{F})$ a k-ésima direção da base canônica do $\mathbb{R}^{m}$. Se $d^{k}$ é uma direção de aresta saindo de $f$, então o algorítmo (6) para $\left(f, d^{k}\right)$ retorna $\theta_{k}=\infty$, e podemos anular o coeficiente $a_{k}$ do corte de Tǔ procurado.

Em outros termos, o corte de Tu乞̆ pode ser feito paralelamente à direção $d^{k}$ de ilimitação da função.

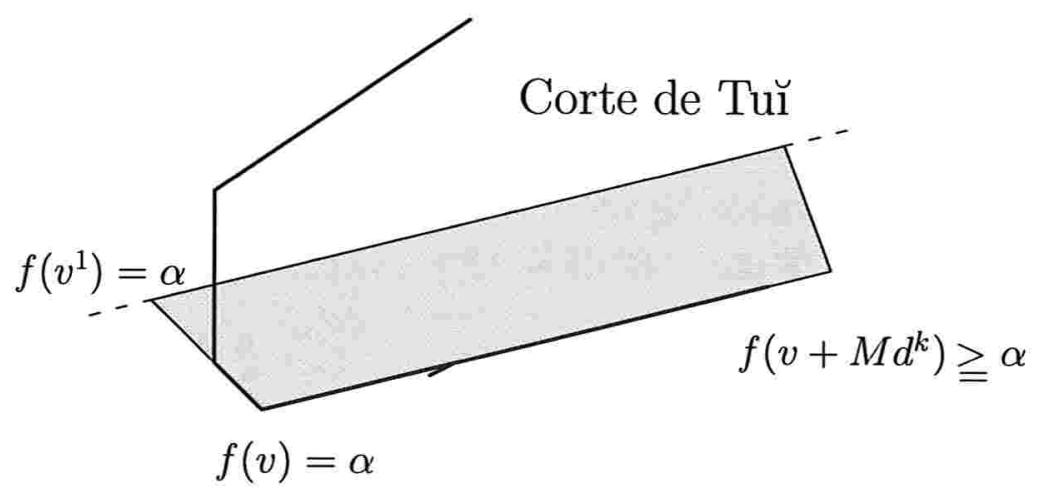

Prova : Sabemos que $d^{k} \in C(\mathcal{F})$. Idealmente, queremos que o corte de Tul elimine todos os pontos da forma $x^{k}=f+\theta_{k} d^{k}$, para $\theta_{k} \geqq 0$. Seja $\langle a, x\rangle \geqq b$ um candidato a corte de Tŭ. Queremos então que

$$
\left\langle a, x^{k}\right\rangle=\langle a, f\rangle+\theta_{k}\left\langle a, d^{k}\right\rangle=\langle a, f\rangle+\theta_{k} a_{k}<b, \forall \theta_{k} \geqq 0,
$$

ou seja, $a_{k}=0$.

Este é exatamente o corte "limite", ao fazer $\theta_{k} \longrightarrow \infty$ na expressão $x^{k}=f+\theta_{k} d^{k}$.

Esta é uma maneira eficiente de introduzir o corte de Tǔ̆ paralelamente ao raio extremal, evitando mal-condicionamento numérico (ao se escolher um ponto "arbitrariamente distante" nesta direção, como recomendado por [Tuĭ 64]).

Se $d^{k}$ é um desvio elementar associado à inclusão do arco $(i, j)$ na árvore $G_{f}^{r}$, a atualização da árvore $G_{f}^{r}$ (e portanto da parcela $f^{r}$ do fluxo $f$ ) nesta direção será dado por 
Definição 4.3 (Atualização de $f^{r}$ : caso normal)

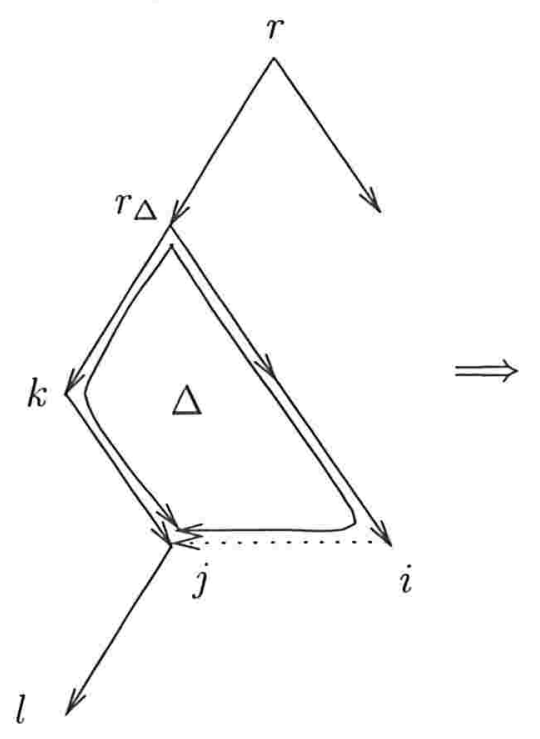

Atualização de $G_{f}^{r}$

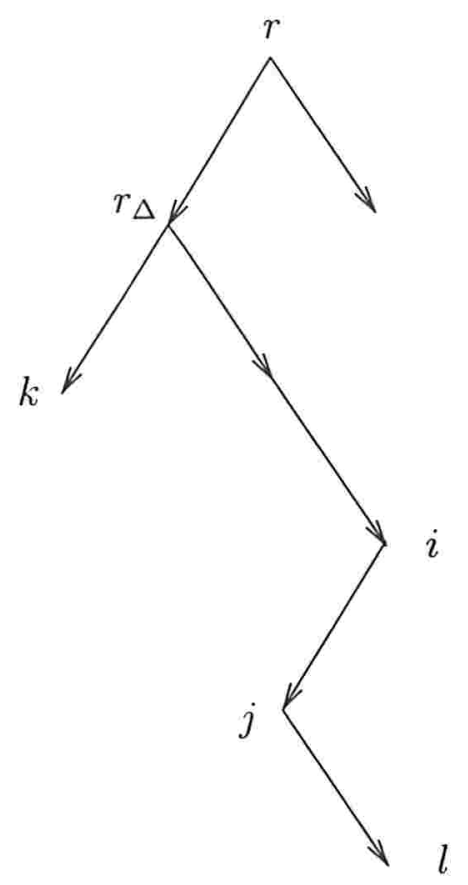

Definição 4.4 (Atualização de $f^{s}$ : caso problemático)

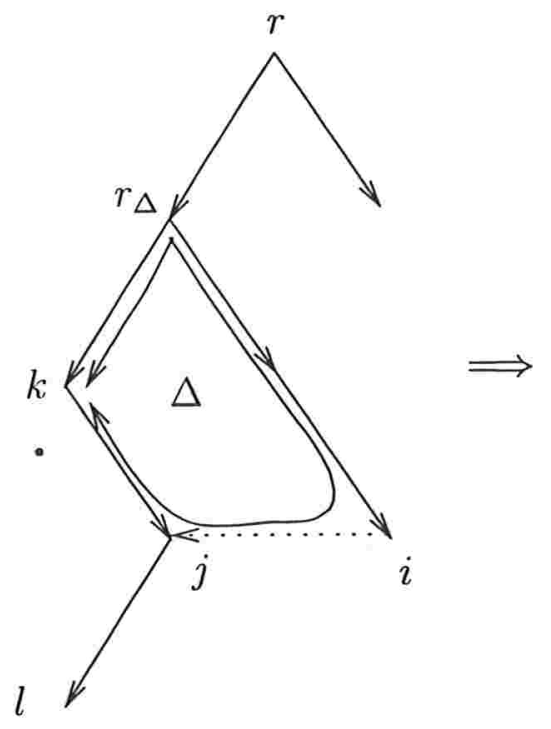

Atualização de $G_{f}^{s}$ :

caso problemático

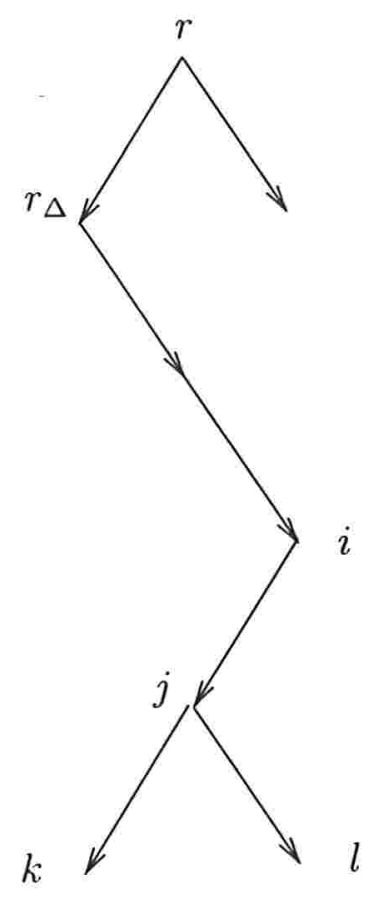

As atualização das outras árvores $G_{f}^{s}$, para $s \neq r$ poderá ser feita sempre que $d^{k}$ for um desvio 
elementar também em relação à árvore $G_{f}^{s}$, e neste caso a atualização de $G_{f}^{s}$ e $f^{s}$ será também análoga ao da figura acima. Pode acontecer, porém, que a direção $d^{k}$ seja uma direção viável em relação a um dos fluxos $f^{s}$, mas $d^{k}$ não é desvio elementar em relação à arvore $G_{f}^{s}$. Neste caso, poderíamos atualizar a árvore $f^{s}$ como na definição (4.4).

Esta atualização (descrita em (4.4)), em geral não causa uma variação de fluxo na direção $d^{k}$, mas em uma outra direção, que depende do arco que é eliminado em (4.4). Basta notar que no último exemplo, o fluxo em $\left\{r_{\Delta}, k\right\}$ sofrerá uma variação de $-q_{r k}-q_{r j}-q_{r l}$, enquanto o fluxo no arco $\{k, j\}$ sofrerá uma variação de $-q_{r j}-q_{r l}+q_{r k}$, e conseqüentemente a direção de atualização não corresponderá a um desvio elementar (proposição (3.39)).

Este processo de atualização não garante sequer que o fluxo atualizado dado pelas árvores $G_{f^{\prime}}^{1}, \ldots, G_{f^{\prime}}^{n}$ seja coerente. O exemplo a seguir ilustra uma aplicação deste procedimento que leva um fluxo coerente a um não-coerente:

\section{Atualização de um fluxo coerente pode não ser coerente:}
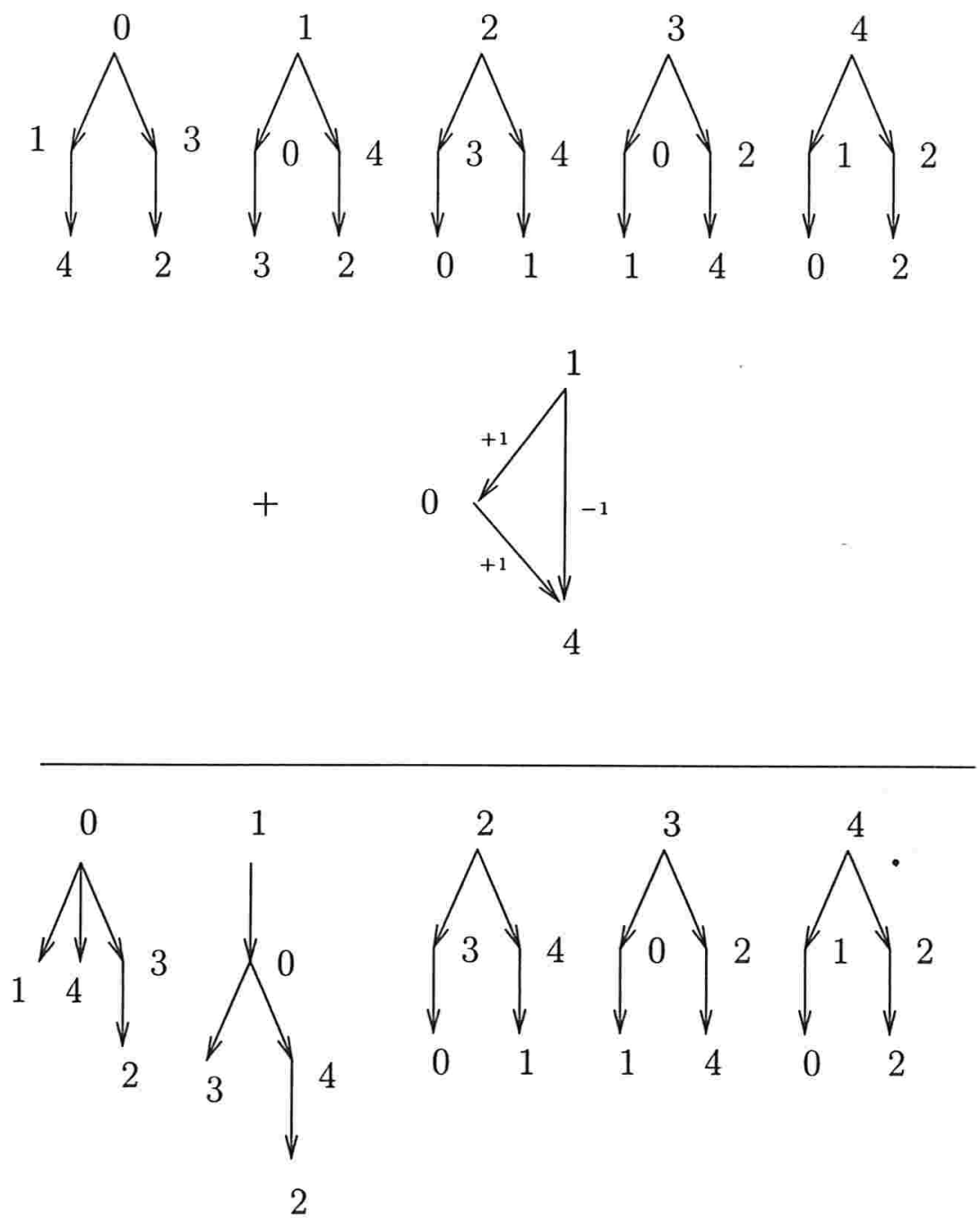

Porém o fluxo obtido sempre corresponde a um vértice do poliedro $\vec{F}^{1} \times \vec{F}^{2} \times \cdots \times \vec{F}^{n}$, e a partir dele ainda podemos construir direções que formam aproximações cônicas do poliedro; e 
continuamos a ter a garantia de que todo mínimo local é um vértice, ainda que este mínimo local tenha sido atingido através de fluxos não-coerentes (proposição (4.1)).

Nos testes computacionais realizados, para todo fluxo coerente e para toda direção $d^{k}$ gerada por (5), este fenômeno não ocorreu; isto é, a partir de um fluxo coerente, a aplicação do procedimento descrito acima sempre gerou outro fluxo coerente. Note que nosso exemplo problemático utiliza uma direção elementar "interior", que é eliminada pelo passo 2 do algoritmo (5):

Direção interior: $d^{k}=\Delta^{e}+h$.

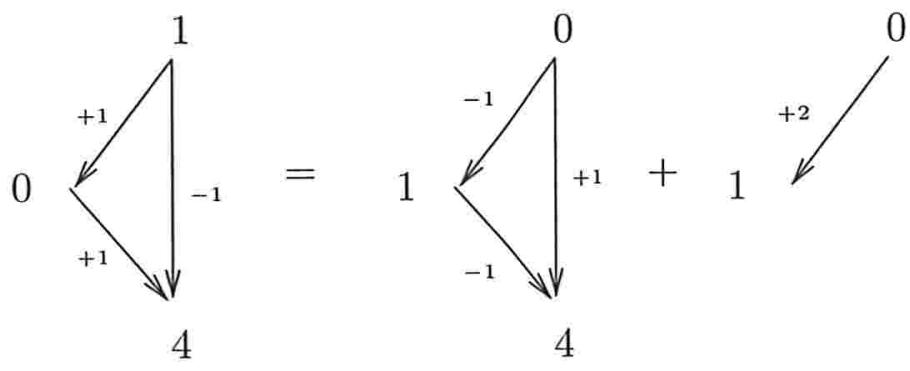

Infelizmente, não conhecemos um exemplo em que este fenômeno ocorra com uma direção devolvida pelo algorítmo (5), nem mesmo um argumento teórico que justifique nossa experiência computacional.

Um pseudo-argumento no caso de $f \in V(\mathcal{F})$ é que as direções $d^{k}$ dadas por (5) são direções de arestas saindo de $f$, e conseqüentemente, nas direções associadas a desvios elementares, é certo que existe um vértice diferente de $f$ naquela direção. O problema formal é que, entre dois vértices, poderia existir um fluxo coerente que não é vértice, como na figura abaixo:

\section{Fluxos coerentes alinhados:}

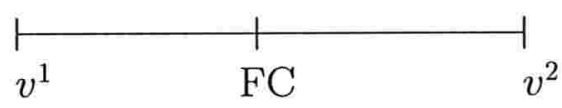

Entendemos, portanto, que esta heurística, da maneira como foi definida, e com os resultados teóricos que dispomos, poderá passar por vértices de $\vec{F}^{1} \times \vec{F}^{2} \times \cdots \times \vec{F}^{n}$ que não são nem mesmo fluxos coerentes.

O passo 4 da heurística (1) pode ser implementado como segue: para cada direção $d^{k}$ a partir de um fluxo $f$, verificamos se a atualização correspondente a esta direção (como definida em (4.3) e (4.4)) deixará de obedecer a alguma restrição introduzida pelos cortes de Tul̆ anteriores. Neste caso, $d^{k}$ não será contabilizada como uma direção viável. Porém, para a identificação de um mínimo local, é necessário que em todas as direções $d^{k}$ saindo de $f$, os pontos $x^{k}$ para o corte de Tŭ tenham valor $D^{*}\left(x^{k}\right)$ superior a $D^{*}(f)$, mesmo nas direções que se tornaram inviáveis.

Esta especificação é a causa do principal ponto fraco da heurística (1). Dependendo da ordem em que os cortes de Tŭ são introduzidos, poderemos atingir um fluxo $f$ que não é mínimo local, mas que também não possui nenhuma aresta viável associada a um fluxo $f^{\prime} \operatorname{com} D^{*}\left(f^{\prime}\right)<D^{*}(f)$. por causa dos cortes de Tư̆. Neste caso, a heurística pára, mesmo que muitos fluxos coerentes ainda não tenham sido visitados. Diríamos que o fluxo coerente ficou "ilhado": 


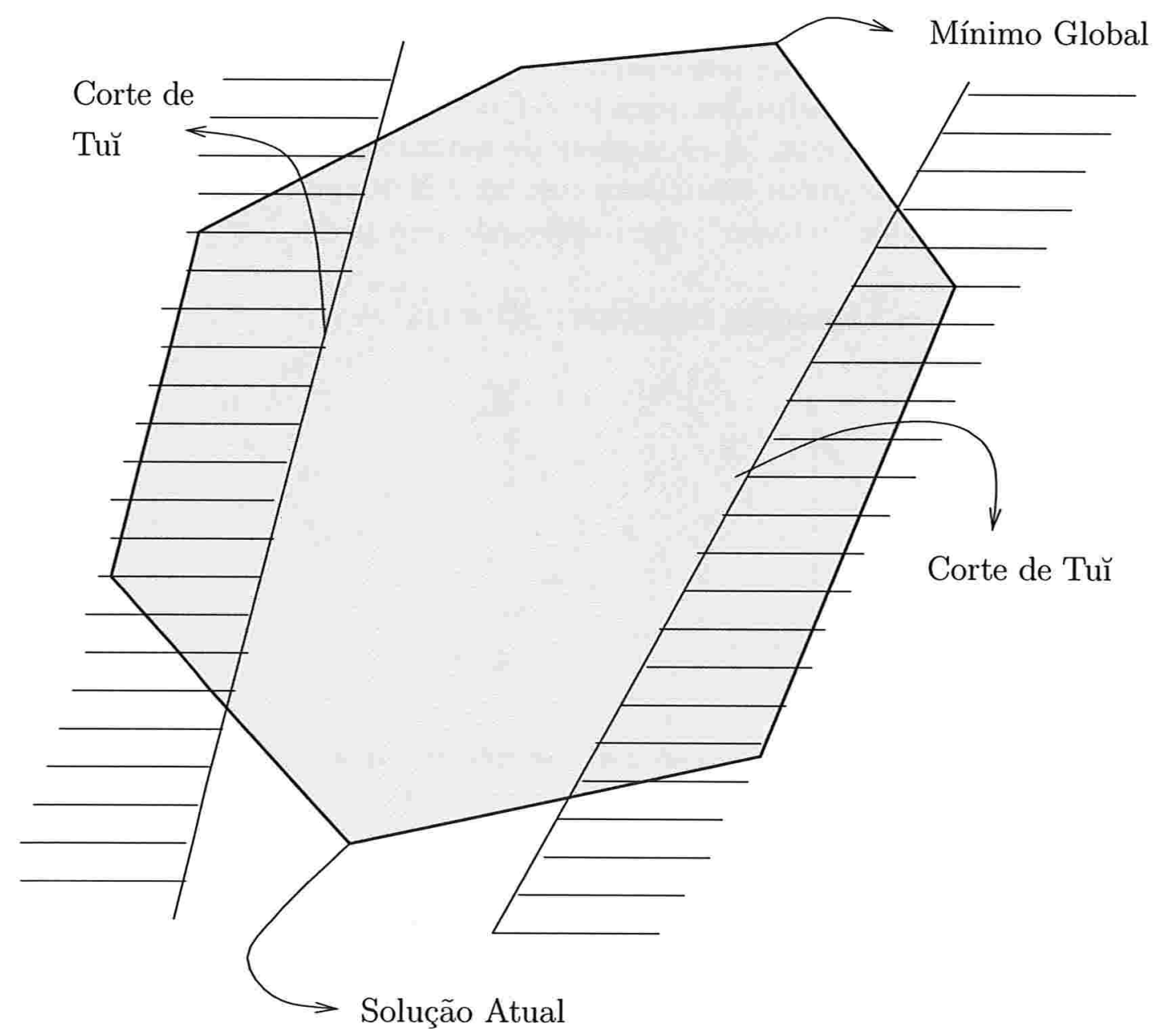

Situação de isolamento nas heurísticas (1) e (2).

A heurística (2) surgiu justamente de uma tentativa de prevenir, ou mais corretamente falando adiar, este estado de isolamento. Nesta heurística, a menos do cálculo do plano de corte de Tuŭ, são utilizadas todas as direções da inicialização do algoritmo (5). As direções que seriam eliminadas no passo 2 do algorítmo (5), correspondem a direções viáveis que apontam para o interior do poliedro; as atualizações nestas direções, como definidas em (4.3) e (4.4), não preservam coerência, como pudemos observar nos testes computacionais realizados. Esta heurística, portanto, permite que a seqüência de fluxos gerados não descreva um "caminho pelas arestas" do poliedro; isto a torna menos "rígida" em relação à heurística (1). 
Heurística 2 (Heurística (2) para o (CFA)) Seja $\varepsilon>0$ fixado.

1. Encontre um fluxo coerente inicial $f^{0}$. Faça Valor Ótimo $=D^{*}\left(f^{0}\right)$, Ótimo $=f^{0}$, Restrições $=\emptyset e i=0$

2. Encontre um mínimo local $z$ a partir de $f^{i}$, utilizando como direções de pesquisa todas as direçôes da inicialização do algoritmo (5). Se isto não for possivel, pare! Caso contrário, este minimo local é um vértice de $\mathcal{F}$ (proposição (4.1)). Se o valor de $D^{*}(z)$ é menor do que Valor Ótimo, atualize Valor Ótimo e Ótimo.

3. Gere as direções de arestas a partir de•z através do algoritmo (5), e calcule o corte de Tŭ gerado por $z$ (2.23), como descrito anteriormente, em particular, ignorando a inviabilidade de direções em relação aos cortes de Tuน̆ já introduzidos. Seja $\langle a, x\rangle \geqq b$ este novo corte.

4. Maximize a função $\langle a, x\rangle$, percorrendo somente fluxos que respeitem todas as restrições do conjunto Restrições, utilizando como direções de pesquisa todas as direções da inicialização do algoritmo (5).

5. Se a solução ótima $x^{*}$ do passo 4 tem valor $\left\langle a, x^{*}\right\rangle>b+\varepsilon$, então faça Restrições $\longrightarrow$ Restrições $\cup\left\{\langle a, x\rangle \geqq b, f^{i+1}=f^{*}, i \longrightarrow i+1\right.$, e volte para o passo 2 . Caso contrário, pare!

Note que a própria crença de que a heurística (2) realmente abra mais possibilidades de busca para o minimizador global, não possui qualquer embasamento formal. Na seção (4.3), mostraremos os testes realizados, nos quais a heurística (2) foi sempre mais bem sucedida do que a heurística (1).

Enfatizamos que uma grande virtude destas heurísticas é o fato de não gerarem novos vértices no poliedro, e conseqüentemente, não estarem sujeitas a problemas de instabilidade numérica provenientes de cortes mal-condicionados (por exemplo, um corte com uma coordenada igual a $10^{-7}$ e uma outra igual a $2^{56}$ ). No caso de demandas inteiras, todos os fluxos visitados serão inteiros (corolário (3.21)), e portanto a única possível fonte de imprecisão numérica será o cálculo da função $D^{*}(\cdot)$ (algoritmo (2)).

\subsection{Tǔ̆-Zwart Aplicado a $\vec{F}^{1} \times \vec{F}^{2} \times \cdots \times \vec{F}^{n}$}

Antes de mais nada, é fundamental estabelecer que a implementação do algoritmo de Tŭ̌-Zwart para o poliedro $\vec{F}^{1} \times \vec{F}^{2} \times \cdots \times \vec{F}^{n}$ teve por objetivo principal fornecer uma medida comparativa da performance (não só de tempo, mas também de acuidade da solução encontrada) para as duas heurísticas. Para a maioria dos exemplos já tínhamos sido capazes de enumerar todo o conjunto $V(\mathcal{F})$, portanto sabíamos a priori a relação entre as soluções obtidas pelas heurísticas e os valores ótimos dos respectivos problemas. 
O poliedro $\vec{F}^{1} \times \vec{F}^{2} \times \cdots \times \vec{F}^{n}$, como foi visto na proposição (3.18) é dado pelo sistema

$$
\left\{\begin{array}{rrrr}
\mathcal{J} f^{1} & & & =b^{1} \\
\mathcal{J} f^{2} & & =b^{2} \\
& & \vdots & \vdots \\
& & \mathcal{J} f^{n} & =b^{n} \\
\left(f^{1}, f^{2}, \ldots, f^{n}\right)^{t} & \geqq 0 .
\end{array}\right.
$$

O algorítmo de Tŭ̌-Zwart exige que o poliedro seja limitado; podemos usar umas das limitações que a proposição (3.20) fornece:

$$
\sum_{e \in \vec{E}} f_{e}^{r} \leqq(n-1) \sum_{s \neq r} q_{r s}, \forall r \in V
$$

ou

$$
\sum_{r \in V} \sum_{e \in \vec{E}} f_{e}^{r} \leqq m \sum_{r \in V} \sum_{s \neq r} q_{r s}
$$

A introdução do primeiro conjunto de restrições, além de prevenir possíveis problemas numéricos (no caso do valor $m \sum_{r \in V} \sum_{s \neq r} q_{r s}$ ser muito grande), possibilita que cada um dos poliedros:

$$
P^{r}=\left\{f^{r} \mid \mathcal{J} f^{r}=b^{r}, f^{r} \geqq 0 \text { e } \sum_{e \in \vec{E}} f_{e}^{r} \leqq(n-1) \sum_{s \neq r} q_{r s}\right\}
$$

seja limitado, o que poderia simplificar qualquer estratégia de decomposição.

A introdução de cortes de Tŭ faria do sistema anterior um novo sistema da forma

$$
\left\{\begin{array}{cccc}
\mathcal{J} f^{1} & & & \\
& \mathcal{J} f^{2} & & b^{1} \\
& & & b^{2} \\
& \ddots & \vdots & \vdots \\
\mathcal{J} f^{n} & & b^{n} \\
L^{1} f^{1}+L^{2} f^{2} \ldots \quad+L^{n} f^{n}+M x^{r} & = & b^{n+1} \\
& \left(f^{1}, f^{2}, \ldots, f^{n}, x^{r}\right)^{t} \geqq 0 . &
\end{array}\right.
$$

Tal configuração sugere imediatamente a tentativa de aplicação do método de Dantzig-Wolfe (seção (1.2.2)); devemos lembrar, porém, que aquele era um método para a solução de problemas de programação linear, e depende fortemente que a função objetivo seja linear (ver, por exemplo, o teorema (1.29)).

O algoritmo de Tuй-Zwart possui, de fato, um subproblema linear. Para este subproblema, Dantzig-Wolfe seria provavelmente a melhor opção de implementação. Porém, os outros passos do algorítmo trabalham com propriedades geométricas do poliedro envolvido. No nosso caso, trabalharíamos com o poliedro definido na forma acima, ou com o Poliedro de Dantzig-Wolfe associado, no espaço das variáveis $\lambda_{k_{r}}^{r}$ onde $r \in V$ e $k_{r}$ é o número total de vértices e de raios extremais associados ao poliedro $\vec{F}^{r}$ ?

A resposta imediata é que o poliedro de Dantzig-Wolfe é inapropriado para o algoritmo de Tư̆-Zwart, pois este precisa explorar, a cada passo, o conjunto de todas as direções que saem de 
um vértice. Note que o número de direções para o poliedro de Dantzig-Wolfe será da ordem do produto $k_{1} k_{2} \cdots k_{n}$. Note que Dantzig-Wolfe aproveita a estrutura linear da função objetivo, para providenciar uma estratégia inteligente de geração de colunas, de tal forma que nunca precisamos conhecer todo o sistema linear do poliedro de Dantzig-Wolfe.

A única opção plausível, portanto, para a implementação de Tu1̆-Zwart utilizando este poliedro, é alternar entre o poliedro de Dantzig-Wolfe para o passo de programação linear do algoritmo de Tŭ, e o poliedro original para todos os demais passos.

Um problema que surge com esta abordagem, é o de relacionar os vértices do poliedro original com os vértices do poliedro de Dantzig-Wolfe. Não seria razoável, após a introdução do corte de Tư̆, ignorar o conhecimento do vértice viável atual, para aplicar Dantzig-Wolfe com inicialização por fase 0. E se existir a possibilidade da solução ótima para Dantzig-Wolfe não corresponder a um vértice do poliedro original, isto é, corresponder a um fluxo no interior relativo da face ótima do poliedro original, teríamos que acrescentar uma rotina para encontrar um vértice ótimo a partir de um não-vértice ótimo.

Entendemos, portanto, que o algoritmo de Dantzig-Wolfe não teria um impacto considerável no algoritmo de Tư̆, se utilizado apenas no passo linear, com todos os cuidados para a alternância entre os dois poliedros. Somente um estudo mais cuidadoso da relação entre a geometria do poliedro original e a do poliedro de Dantzig-Wolfe, poderia dizer se tais possibilidades descritas no parágrafo anterior são fictícias ou não, e a partir daí elaborar uma interação consciente e coerente entre Dantzig-Wolfe e Tŭ-Zwart.

Nossa abordagem para o algoritmo de Tŭ-Zwart prevê, portanto, a aplicação ao poliedro $\vec{F}^{1} \times \vec{F}^{2} \times \cdots \times \vec{F}^{n}$ descrito acima.

Note que para esta aplicação, todos os elementos de implementação necessários já foram discutidos. A inicialização pode ser feita por simplex fase 0 . A exploração de vértices vizinhos já foi caracterizada para o caso não-degenerado (proposição (1.34)), assim como a determinação do corte de Tư̆ (seção (2.5)).

Para o caso de aparecimento de um vértice degenerado, poderíamos aplicar um método de perturbações (como descrito, por exemplo, em [Bazaraa \& Jarvis 77], [Dantzig 63], [Luenberger 73]). Assim como no caso de Dantzig-Wolfe, alguma investigação seria necessária para garantir que na implementação das perturbações, pudéssemos gerar todas as direções saindo do vértice degenerado. Frisamos que esta é uma condição sine qua non para o algoritmo de Tuı̆-Zwart.

Outra estratégia é tentar introduzir um corte, que elimine apenas o vértice $v$ degenerado, sem eliminar nenhum outro vértice. Um corte com esta propriedade pode ser obtido pela expressão

$$
\sum_{j \notin B} x_{j}+\sum_{j \in B: v_{j}=0} x_{j} \geqq \varepsilon
$$

onde $B$ é uma base associada ao vértice degenerado, e $\varepsilon$ é um número positivo arbitrariamente pequeno. É claro que para um $\varepsilon$ arbitrário,existe a possibilidade de que este corte elimine o mínimo global para o problema, antes que este tenha sido visitado pelo algorítmo.

Observamos, nos exemplos computacionais, que o corte de Tư̆ na sua forma original pode introduzir degenerescência, e mesmo após a introdução dos cortes acima mencionados, pudemos encontrar degenerescência. Sobre bases empíricas somennte, verificamos que perturbar a restrição de Tŭ antes de introduzi-la no sistema emgeral reduzia o número de ocorrências de degenerescência 
drasticamente. A restrição de Tŭ ficaria portanto

$$
\sum_{i \notin B} \frac{1}{\theta_{k}} x_{k} \geqq 1+\varepsilon
$$

onde $\theta^{k}$ são os múltiplos convenientes das direções de arestas, como explicado na seção (2.5).

Nossa implementação para o algoritmo de Tŭ-Zwart aplicado ao (CFA), com as observações acima, teve resultados muito ruins. Em todos os casos o algoritmo parou por instabilidade numérica, antes de esgotar o poliedro, isto é, antes que um dos subproblemas lineares tivessem acusado que a sua solução ótima não atravessa o corte de Tŭ. Em três dos seis exemplos para os quais o algoritmo foi testado, o mínimo global não foi encontrado. Chamamos a atenção para um comentário de [Zwart 74] a respeito desta versão do algorítmo, reportando também grande ineficiência na implementação; Zwart advoga a vantagem da implementação do método original (ver comentário no início da seção (2.4.1)), que não escolhemos como algorítmo de trabalho pela aparente enorme quantidade de subproblemas gerados em cada passo.

\subsection{Resultados Computacionais e Análise Comparativa}

Nossa implementação para as heurísticas usa como interface de entrada três arquivos; um contém a descrição do grafo, outro a descrição das demandas, e o último contém os coeficientes da função custo $D(\cdot)(2.8)$. Os parâmetros $T_{\text {MAX }}$ e o expoente $\alpha$ da função custo são passados pela linha de comando.

O problema associado ao grafo

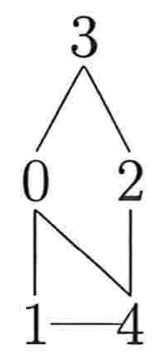

com demandas $q_{r s}=1, \forall r, s, r \neq s$ e função custo dada por

$$
D(c)=\sum_{e=1}^{6} \sqrt{c_{e}}
$$

é representado pelos arquivos: 


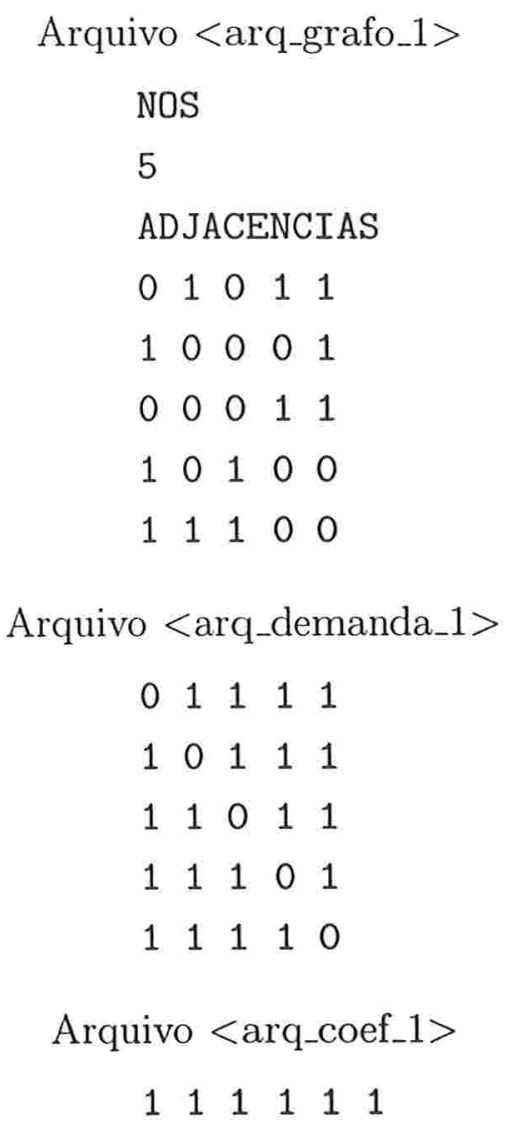

Uma das opções de saída do programa é um relatório, utilizando a linguagem $\mathrm{AT}_{\mathrm{E} X 2 \mathrm{e}}$, que inclui os desenhos das árvores $G_{f}^{1}, \ldots, G_{f}^{n}$. Para exemplificar, incluímos um destes relatórios completos, na próxima página.

Neste relatório, as únicas alterações manuais são a média de fluxos coerentes cortados por restrição (última linha da tabela), e o valor ótimo do problema; estes dados são gerados por um outro programa, que faz a enumeração completa dos fluxos coerentes no poliedro $\mathcal{F}$. O poliedro deste exemplo, imerso no $\mathbb{R}^{1} 0$, tem 556 fluxos coerentes.

Explicaremos a seguir cada uma das estatísticas que foram extraídas dos testes. Estas compõem a tabela no início do relatório.

A função <mínimo_local > corresponde ao passo 2 das heurísticas (1) e (2). Para esta função, foram contabilizados o número de chamadas (isto é, o número de mínimos locais encontrados), a média do número de fluxos pesquisados em cada chamada, e o número médio de direções elementares saindo de cada fluxo (ou seja, não contabilizando os raios extremais do cone).

A função <corta_poliedro> corresponde ao cálculo do corte de Tul̆ e à solução do problema linear associado. Para esta, contabilizamos o número de chamadas (que corresponde ao número de cortes introduzidos), a média do número de pontos $x^{k}$ utilizados no cálculo da restrição (lembremos que os raios extremais não são utilizados no cálculo da restrição, por (4.2)), a média do número de fluxos pesquisados por chamada, e a média de direções não-triviais pesquisadas por fluxo.

A função <ca > corresponde ao cálculo da função $D^{*}(\cdot)$; o número de chamadas contabilizado tem um significado pouco claro para a análise de performance da heurística, por causa das buscas uni-direcionais efetuadas. 
Exemplo de relatório gerado pelas heurísticas (1) e (2)

\begin{tabular}{|c|c|c|}
\hline \multicolumn{3}{|c|}{ HEURISTICA 2} \\
\hline Função <mínimo_local> & $\begin{array}{l}\text { \# de chamadas } \\
\text { \# de fluxos pesquisados (média) } \\
\text { \# de direções pesquisadas (por fluxo) }\end{array}$ & $\begin{array}{cc}: & 9 \\
: & 4.56 \\
: & 6.90\end{array}$ \\
\hline Função <corta_poliedro> & $\begin{array}{l}\text { \# de chamadas } \\
\text { \# de pontos para o cálculo da restrição } \\
\text { \# de fluxos pesquisados (média) } \\
\text { \# de direções pesquisadas (por fluxo) }\end{array}$ & $\begin{array}{cc}: & 8 \\
: & 3.00 \\
: & 3.38 \\
: & 1.11\end{array}$ \\
\hline Função <ca > & \# de chamadas & $: 790$ \\
\hline Fase 0 do simplex & \# de chamadas (por fluxo) & $: 29.11$ \\
\hline
\end{tabular}

Parâmetros Do Problema: $\alpha=0.50, T_{\mathrm{MAX}}=1.00, q=\left[\begin{array}{llllllll}0.00 & 1.00 & 1.00 & 1.00 & 1.00 & 1.00 & 1.00 & 1.00 \\ 1.00 & 0.00 & 1.00 & 1.00 & 1.00 & 1.00 & 1.00 & 1.00 \\ 1.00 & 1.00 & 0.00 & 1.00 & 1.00 & 1.00 & 1.00 & 1.00 \\ 1.00 & 1.00 & 1.00 & 0.00 & 1.00 & 1.00 & 1.00 & 1.00 \\ 1.00 & 1.00 & 1.00 & 1.00 & 0.00 & 1.00 & 1.00 & 1.00 \\ 1.00 & 1.00 & 1.00 & 1.00 & 1.00 & 0.00 & 1.00 & 1.00 \\ 1.00 & 1.00 & 1.00 & 1.00 & 1.00 & 1.00 & 0.00 & 1.00 \\ 1.00 & 1.00 & 1.00 & 1.00 & 1.00 & 1.00 & 1.00 & 0.00\end{array}\right]$, e

$D(c)=\sum_{i=1}^{10} l_{i} c_{i}^{\alpha}=1.00 c_{03}^{0.50}+1.00 c_{12}^{0.50}+1.00 c_{13}^{0.50}+1.00 c_{24}^{0.50}+1.00 c_{25}^{0.50}+1.00 c_{34}^{0.50}+1.00 c_{35}^{0.50}+1.00 c_{47}^{0.50}+1.00 c_{56}^{0.50}+1.00 c_{57}^{0.50}$.

Roteamento Associado À Solução Encontrada:
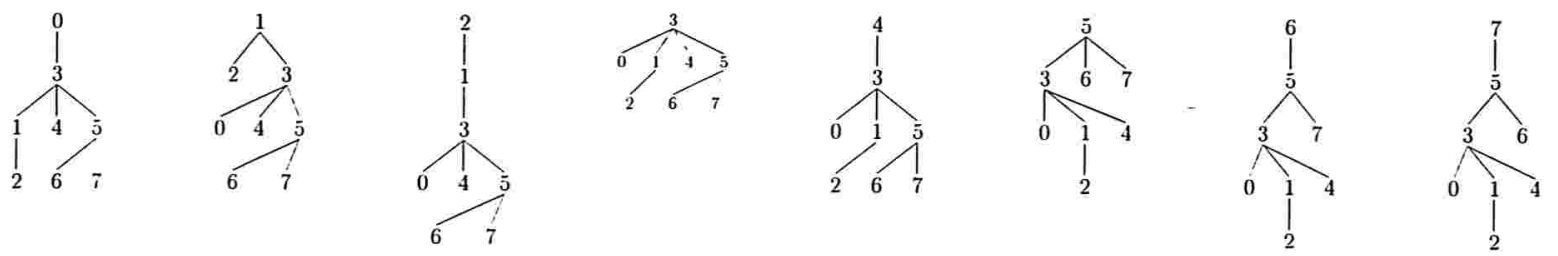

Fluxo Total Projetado:

$$
f=\left[\begin{array}{cc}
14.00 & 0 \leftrightarrow 3 \\
14.00 & 1 \leftrightarrow 2 \\
24.00 & 1 \leftrightarrow 3 \\
0.00 & 2 \leftrightarrow 4 \\
0.00 & 2 \leftrightarrow 5 \\
14.00 & 3 \leftrightarrow 4 \\
30.00 & 3 \leftrightarrow 5 \\
0.00 & 4 \leftrightarrow 7 \\
14.00 & 5 \leftrightarrow 6 \\
14.00 & 5 \leftrightarrow 7
\end{array}\right]
$$

Melhor Valor Encontrado:

$$
D^{*}(f)=30.83811
$$

Valor ótimo para o problema: 29.80151

Tempo Gasto : $0 \mathrm{~m} 1.176 \mathrm{~s}$ 
O número de chamadas da fase 0 do simplex, corresponde ao número de vezes que este procedimento foi utilizado para testar se uma dada direção $d^{k}$ era positivamente linearmente independente das outras (passo 2 do algorítmo (5)).

A média de fluxos coerentes cortados por restrição é uma das medidas mais interessantes de performance do algoritmo, pois dá uma idéia da "profundidade" média dos cortes.

Os testes realizados utilizaram os problemas descritos no final desta seção. Além da especificação destes problemas, incluímos os roteamentos associados às melhores soluções obtidas pelas heurísticas. Os resultados dos testes foram organizados nas tabelas a seguir.

\begin{tabular}{|c|c|c|c|c|c|c|c|c|c|c|c|c|c|}
\hline \multicolumn{14}{|c|}{ Heurística 1} \\
\hline \multirow[b]{2}{*}{$\begin{array}{l}0 \\
\frac{0}{0} \\
\frac{0}{0} \\
3 \\
0\end{array}$} & \multirow[b]{2}{*}{ 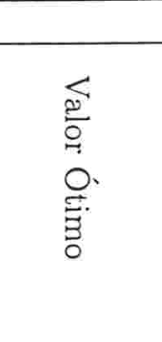 } & \multicolumn{3}{|c|}{$<$ mínimo_local $>$} & \multicolumn{4}{|c|}{$\langle$ corta_poliedro $>$} & \multirow[b]{2}{*}{$\hat{\widehat{\delta}}$} & \multirow[b]{2}{*}{$\begin{array}{l}\hat{A} \\
\hat{\mathscr{E}_{0}} \\
\hat{0} \\
\hat{V}\end{array}$} & \multirow[b]{2}{*}{ 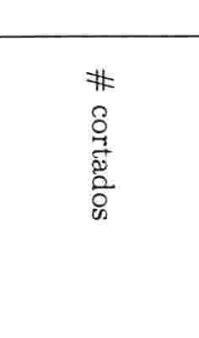 } & \multirow[b]{2}{*}{ 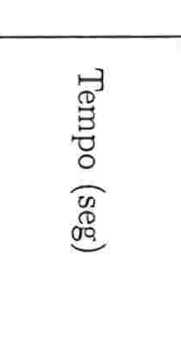 } & \multirow[b]{2}{*}{ 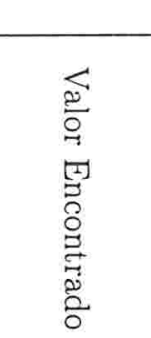 } \\
\hline & & 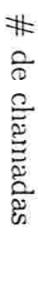 & $\begin{array}{l}\# \\
\# \\
\stackrel{0}{0} \\
\overrightarrow{\vec{Z}} \\
\overrightarrow{0} \\
0\end{array}$ & 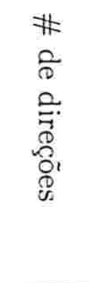 & 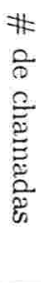 & 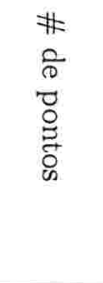 & 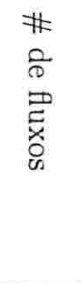 & 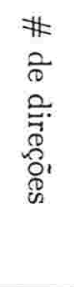 & & & & & \\
\hline 1 & 13.08759 & 7 & 2.00 & 5.14 & 7 & 2.57 & 2.71 & 1.42 & 354 & 15.46 & 9.29 & $0.41 \mathrm{~s}$ & 13.08759 \\
\hline 2 & 35.26519 & 3 & 1.33 & 6.00 & 2 & 2.00 & 3.50 & 1.60 & 90 & 16.00 & 12.00 & $0.19 \mathrm{~s}$ & 35.26519 \\
\hline 3 & 19.63334 & 7 & 2.00 & 5.14 & 7 & 2.57 & 2.71 & 1.42 & 383 & 15.46 & 8.43 & $0.41 \mathrm{~s}$ & 19.63334 \\
\hline 4 & 53.07584 & 3 & 1.33 & 6.00 & 2 & 2.00 & 3.50 & 1.60 & 98 & 16.00 & 12.00 & $0.17 \mathrm{~s}$ & 53.07584 \\
\hline 5 & 29.80151 & 5 & 2.60 & 8.46 & 4 & 3.00 & 3.00 & 1.12 & 313 & 26.95 & 26.75 & $1.956 \mathrm{~s}$ & 30.83811 \\
\hline 6 & 29.80151 & 7 & 2.71 & 10.00 & 6 & 5.00 & 3.50 & 1.07 & 673 & 40.65 & 845.00 & $7.996 \mathrm{~s}$ & 29.80151 \\
\hline 7 & $27.78039 ?$ & 2 & 3.00 & 17.50 & 1 & 21.00 & 5.00 & 1.00 & 430 & 136.80 & $>201414.00$ & $2 \mathrm{~m} 26.125 \mathrm{~s}$ & 27.78039 \\
\hline
\end{tabular}

\begin{tabular}{|c|c|c|c|c|c|c|c|c|c|c|c|c|c|}
\hline \multicolumn{14}{|c|}{ Heurística 2} \\
\hline \multirow[b]{2}{*}{ 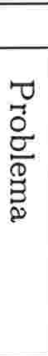 } & \multirow[b]{2}{*}{ 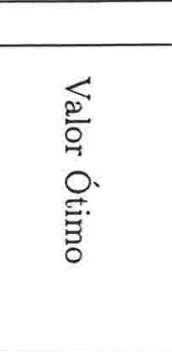 } & \multicolumn{3}{|c|}{$<$ mínimo_local $>$} & \multicolumn{4}{|c|}{$<$ corta_poliedro $>$} & \multirow[b]{2}{*}{$\hat{\hat{\wp}}$} & \multirow[b]{2}{*}{ 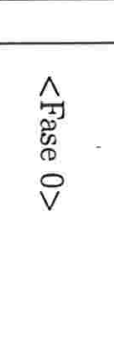 } & \multirow[b]{2}{*}{ 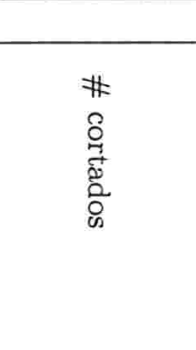 } & \multirow[b]{2}{*}{$\begin{array}{l}\hat{\theta} \\
3 \\
3 \\
0 \\
0 \\
00 \\
\mathbb{0} \\
0\end{array}$} & \multirow[b]{2}{*}{$\begin{array}{l}\stackrel{0}{\circ} \\
\stackrel{0}{0} \\
\stackrel{0}{0} \\
\stackrel{0}{0} \\
\stackrel{0}{0}\end{array}$} \\
\hline & & 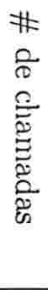 & 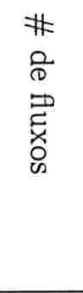 & 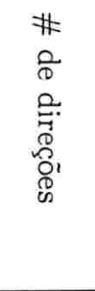 & 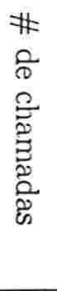 & $\begin{array}{l}\# \\
0 \\
0 \\
0 \\
0 \\
0 \\
0 \\
0 \\
0\end{array}$ & $\begin{array}{l}\# \\
0 \\
0 \\
\stackrel{0}{E} \\
\hat{0} \\
0\end{array}$ & 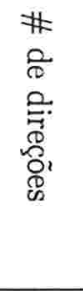 & & & & & \\
\hline 1 & 13.08759 & 10 & 3.00 & 6.60 & 9 & 2.44 & 2.67 & 1.20 & 612 & 18.20 & 8.11 & $0.33 \mathrm{~s}$ & 13.08759 \\
\hline 2 & 35.26519 & 5 & 2.20 & 8.00 & 4 & 2.00 & 2.00 & 1.50 & 249 & 18.40 & 11.50 & $0.17 \mathrm{~s}$ & 35.26519 \\
\hline 3 & 19.63334 & 9 & 2.78 & 6.84 & 8 & 2.50 & 2.62 & 1.69 & 579 & 18.89 & 7.75 & $0.29 \mathrm{~s}$ & 19.63334 \\
\hline 4 & 53.07584 & 5 & 2.20 & 8.00 & 4 & 2.00 & 2.00 & 1.50 & 264 & 18.40 & 10.25 & $0.17 \mathrm{~s}$ & 53.07584 \\
\hline 5 & 29.80151 & 9 & 4.56 & 6.90 & 8 & 3.00 & 3.38 & 1.11 & 790 & 29.11 & 36.00 & $1.176 \mathrm{~s}$ & 30.83811 \\
\hline 6 & 29.80151 & 11 & 5.00 & 10.11 & 10 & 5.00 & 3.50 & 1.04 & 1505 & 43.82 & 1613.60 & $3.476 \mathrm{~s}$ & 29.80151 \\
\hline 7 & $27.78039 ?$ & 2 & 1.00 & 89.00 & 1 & 21.00 & 2.00 & 1.00 & 550 & 117.00 & $>201414.00$ & $19.946 \mathrm{~s}$ & 27.78039 \\
\hline
\end{tabular}

O primeiro fato que chama a atenção, é que para todas as soluções obtidas, $G_{f}$ é uma árvore. Isto nos motiva a procurar possíveis relações entre fluxos dados por árvores (que são vértices, pela proposição (3.33)), e mínimos locais (definição (2.21)).

Notamos também destas duas tabelas, que os tempos de execução para a heurística (2) foram menores dos que os tempos da heurística (1), em todos os casos testados. Além disso, a solução obtida pelas duas heurísticas em todos os casos foi a mesma.

A medida associada ao número de fluxos coerentes eliminados por cada corte de Tŭ pode ser vista percentualmente. Para os quatro primeiros problemas, cada corte de Tŭ eliminou, no 
mínimo, $19.78 \%$ de todos os fluxos coerentes no poliedro associado. Para o problema 5 este índice é de $4.81 \%$, e para o problema $6,1.10 \%$. Para o problema 7 não conhecemos o total de fluxos coerentes, e portanto não pudemos obter esta medida. Note que estes índices se referem ao total de fluxos coerentes; uma medida mais interessante seria o número de fluxos coerentes eliminados pelo $i$-ésimo corte de Tư̆, que ainda não havia sido eliminado nos cortes $1, \ldots, i-1$.

Definição 4.5 ((CFA) : Problema 1)

Grafo:

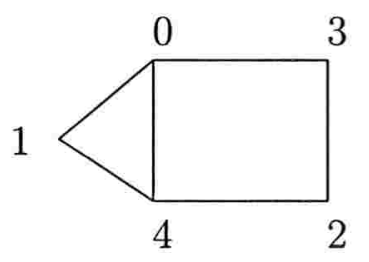

Parâmetros Do Problema: $\alpha=0.50, T_{M A X}=1.00, q_{r s}=1, \forall r \neq s, e$

$$
D(c)=\sum_{i=1}^{6} l_{i} c_{i}^{\alpha}=1.00 c_{01}^{0.50}+1.00 c_{03}^{0.50}+1.00 c_{04}^{0.50}+1.00 c_{14}^{0.50}+1.00 c_{23}^{0.50}+1.00 c_{24}^{0.50} .
$$

O poliedro $\mathcal{F} \subset \mathbb{R}^{6}$ tem 41 fluxos coerentes.

Roteamento Associado À Solução Encontrada:
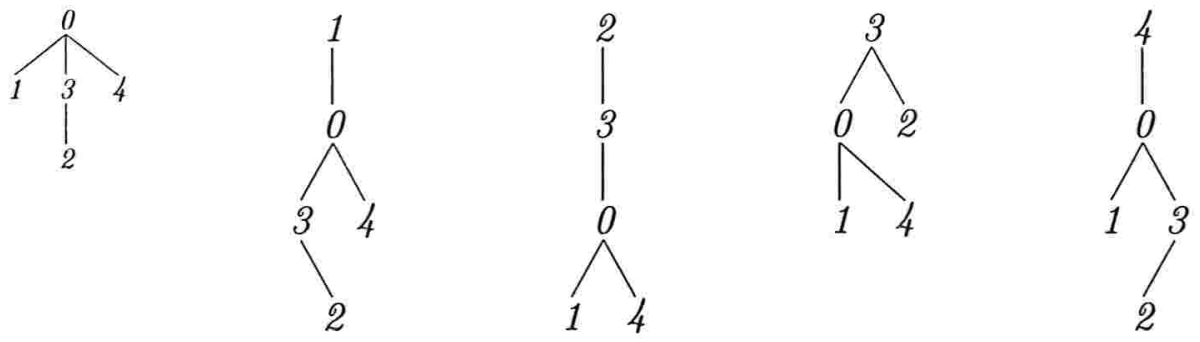

Fluxo Total Projetado:

$$
f=\left[\begin{array}{cc}
8.00 & 0 \leftrightarrow 1 \\
12.00 & 0 \leftrightarrow 3 \\
8.00 & 0 \leftrightarrow 4 \\
0.00 & 1 \leftrightarrow 4 \\
8.00 & 2 \leftrightarrow 3 \\
0.00 & 2 \leftrightarrow 4
\end{array}\right]
$$


Definição 4.6 ((CFA) : Problema 2) $G=$ grafo do problema (4.5).

Parâmetros Do Problema: os mesmos de (4.5).

$$
D(c)=\sum_{i=1}^{6} l_{i} c_{i}^{\alpha}=1.00 c_{01}^{0.50}+2.00 c_{03}^{0.50}+3.00 c_{04}^{0.50}+4.00 c_{14}^{0.50}+5.00 c_{23}^{0.50}+6.00 c_{24}^{0.50} .
$$

O poliedro $\mathcal{F} \subset \mathbb{R}^{6}$ tem 41 fluxos coerentes.

Roteamento Associado À Soluçâo Encontrada: idêntico ao de (4.5).

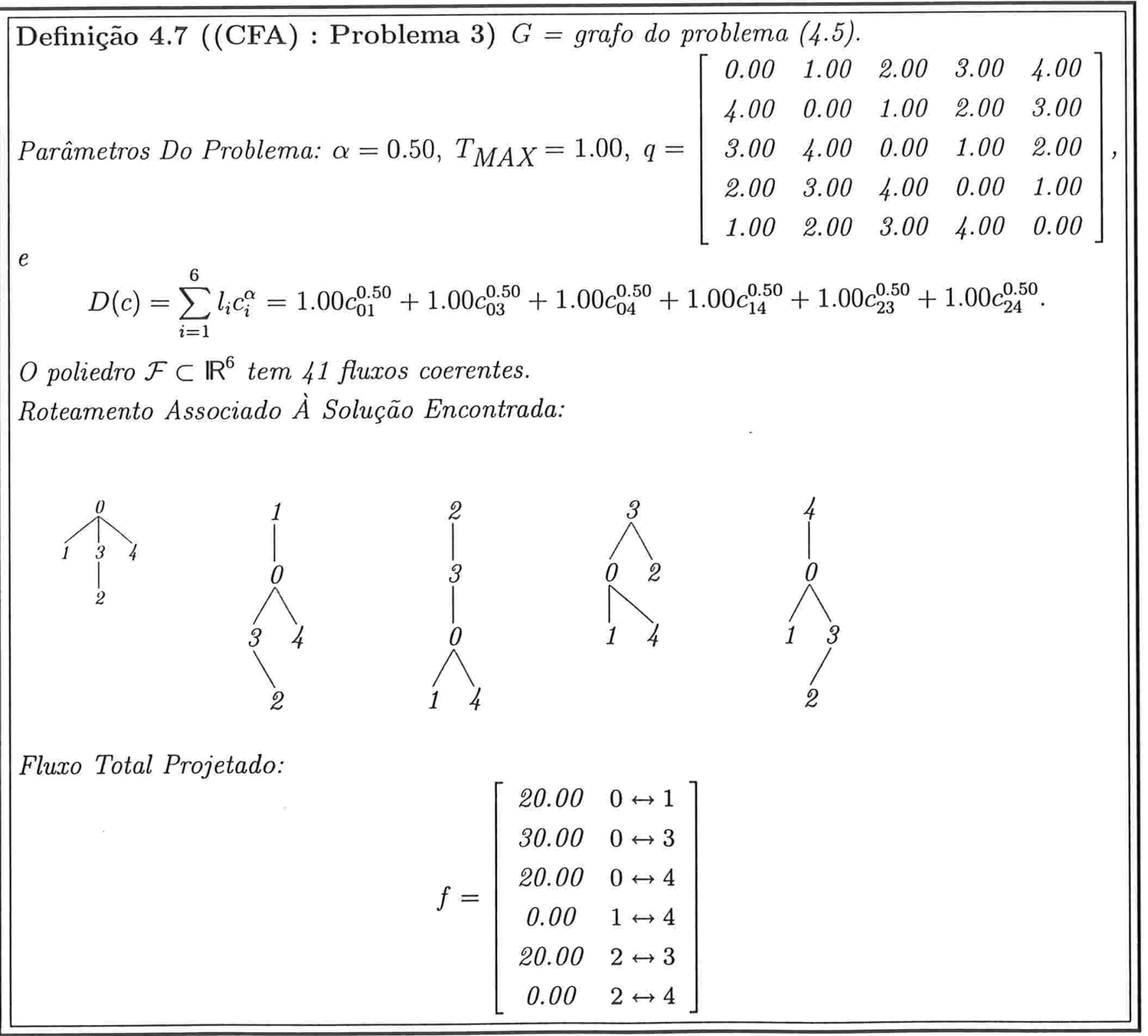


Definição 4.8 ((CFA) : Problema 4) $G=$ grafo do problema (4.5).

Parâmetros Do Problema: os mesmos de (4.7).

$$
D(c)=\sum_{i=1}^{6} l_{i} c_{i}^{\alpha}=1.00 c_{01}^{0.50}+2.00 c_{03}^{0.50}+3.00 c_{04}^{0.50}+4.00 c_{14}^{0.50}+5.00 c_{23}^{0.50}+6.00 c_{24}^{0.50} .
$$

$O$ poliedro $\mathcal{F} \subset \mathbb{R}^{6}$ tem 41 fluxos coerentes.

Roteamento Associado À Solução Encontrada: idêntico ao de (4.7). 
Definição 4.9 ((CFA) : Problema 5)

Grafo:

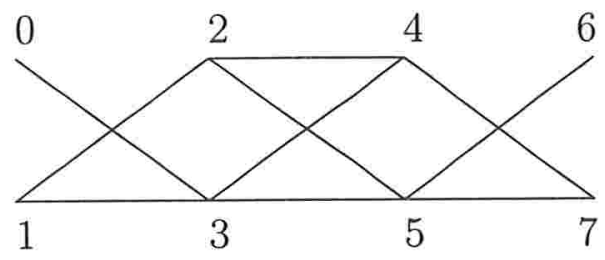

Parâmetros Do Problema: $\alpha=0.50, T_{M A X}=1.00, q_{r s}=1, \forall r \neq s, e$

$$
D(c)=\sum_{i=1}^{10} 1.00 c_{i}^{\alpha} .
$$

O poliedro $\mathcal{F} \subset \mathbb{R}^{10}$ tem 556 fluxos coerentes.

Roteamento Associado À Solução Encontrada:
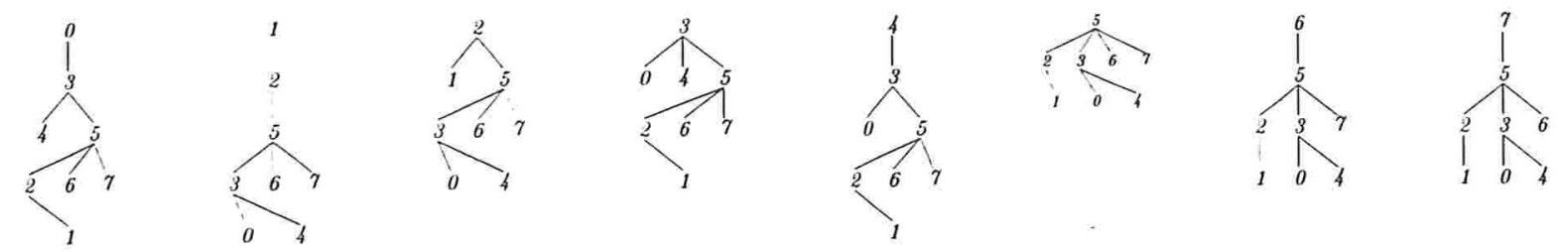

Fluxo Total Projetado:

$$
f=\left[\begin{array}{cc}
14.00 & 0 \leftrightarrow 3 \\
14.00 & 1 \leftrightarrow 2 \\
0.00 & 1 \leftrightarrow 3 \\
0.00 & 2 \leftrightarrow 4 \\
24.00 & 2 \leftrightarrow 5 \\
14.00 & 3 \leftrightarrow 4 \\
30.00 & 3 \leftrightarrow 5 \\
0.00 & 4 \leftrightarrow 7 \\
14.00 & 5 \leftrightarrow 6 \\
14.00 & 5 \leftrightarrow 7
\end{array}\right]
$$


Definição 4.10 ((CFA) : Problema 6)

Grafo:

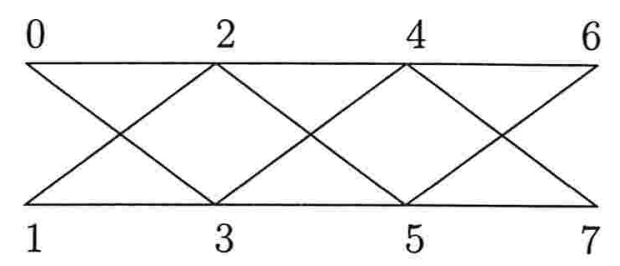

Parâmetros Do Problema: os mesmos de (4.9).

$$
D(c)=\sum_{i=1}^{12} 1.00 c_{i}^{\alpha} .
$$

O poliedro $\mathcal{F} \subset \mathbb{R}^{12}$ tem 76912 fluxos coerentes.

Roteamento Associado À Solução Encontrada:
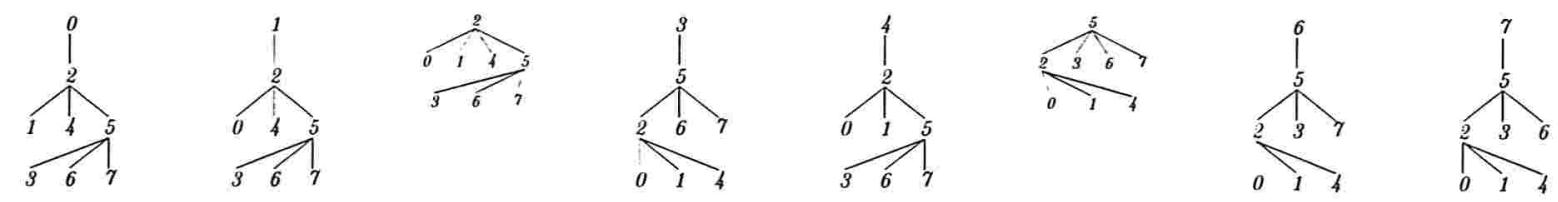

Fluxo Total Projetado:

$$
f=\left[\begin{array}{cc}
14.00 & 0 \leftrightarrow 2 \\
0.00 & 0 \leftrightarrow 3 \\
14.00 & 1 \leftrightarrow 2 \\
0.00 & 1 \leftrightarrow 3 \\
14.00 & 2 \leftrightarrow 4 \\
32.00 & 2 \leftrightarrow 5 \\
0.00 & 3 \leftrightarrow 4 \\
14.00 & 3 \leftrightarrow 5 \\
0.00 & 4 \leftrightarrow 6 \\
0.00 & 4 \leftrightarrow 7 \\
14.00 & 5 \leftrightarrow 6 \\
14.00 & 5 \leftrightarrow 7
\end{array}\right]
$$


Definição 4.11 ((CFA) : Problema 7) Grafo $=K_{8}$.

Parâmetros Do Problema: os mesmos de (4.9).

$$
D(c)=\sum_{i=1}^{28} 1.00 c_{i}^{\alpha} .
$$

$O$ poliedro $\mathcal{F} \subset \mathbb{R}^{28}$ tem mais de 215096 fluxos coerentes.

Roteamento Associado À Solução Encontrada:<smiles>C1CCCCC1</smiles><smiles>CC(C)C</smiles><smiles>CC(C)C</smiles><smiles>CCCCC</smiles><smiles>CCCCCCC</smiles><smiles>CCC(C)C</smiles><smiles>CC(C)=C(C)C</smiles><smiles>CC=C(C)C</smiles>

Fluxo Total Projetado:

$$
\begin{aligned}
& {\left[\begin{array}{ll}
14.00 & 0-1
\end{array}\right]} \\
& 14.00 \quad 0 \multimap 2 \\
& 14.00 \quad 0 \multimap 3 \\
& 14.00 \quad 0-4 \\
& 14.00 \quad 0 \rightarrow 5 \\
& 14.00 \quad 0 \div 6 \\
& 14.00 \quad 0 \leftarrow 7 \\
& 0.00 \quad 1-2 \\
& 0.00 \quad 1 \div 3 \\
& 0.00 \quad 1 \multimap 4 \\
& 0.00 \quad 1-5 \\
& 0.00 \quad 1 \rightarrow 6 \\
& 0.00 \quad 1-7 \\
& 0.00 \quad 2 \rightarrow 3 \\
& 0.00 \quad 2 \leftarrow 4 \\
& 0.00 \quad 2-5 \\
& 0.00 \quad 2 \leftrightarrow 6 \\
& 0.00 \quad 2-7 \\
& 0.00 \quad 3 \multimap 4 \\
& 0.00 \quad 3 \rightarrow 5 \\
& 0.00 \quad 3 \rightarrow 6 \\
& 0.00 \quad 3 \rightarrow 7 \\
& 0.00 \quad 4 \multimap 5 \\
& 0.00 \quad 4 \div 6 \\
& 0.00 \quad 4-7
\end{aligned}
$$




\section{Capítulo 5}

\section{Conclusões e Trabalho Futuro}

Os resultados aparentemente bons das duas heurísticas, e as dificuldades encontradas na implementação do algorítmo no poliedro de alta dimensão, nos motivam naturalmente a procurar novas melhorias para as heurísticas e para a implementação do algorítmo.

Para as primeiras, seria muito interessante pesquisar estratégias para vencer o impasse do critério de parada; não sabemos a priori se a heurística parou e todos os vértices do poliedro original foram realmente cortados, ou se o vértice ótimo ficou perdido em alguma região "inatingível" do poliedro, por causa das restrições dos cortes de Tǔ̆. Além disso, no que diz respeito à atualização de fluxos coerentes nas direções geradas pelo algoritmo (5), é natural perguntar se no caso da atualização de um vértice na direção de uma das arestas saindo dele, é possível garantir que a atualização descrita na seção (4.1) preserva coerência.

Para o algoritmo, tentar aplicar técnicas mais sofisticadas de controle numérico, como normalização das colunas, ou ainda pesquisar mais a fundo a possibilidade de interação entre a técnicas de Dantzig-Wolfe e de perturbações e o algorítmo de Tŭ-Zwart, seriam possíveis abordagens. Outra possibilidade é a implementação do método original de [Zwart 74], cujos experimentos computacionais descritos pelo próprio autor tiveram melhores resultados.

Em relação ao poliedro $\mathcal{F}$, a pergunta que nos parece mais interessante é: qual a condição "mínima" que precisamos impôr a um fluxo coerente para que este seja um vértice?

A caracterização completa das facetas de $\mathcal{F}$ seria outra tentativa interessante. Sabemos que o algoritmo de Falk-Hoffman (algorítmo (4)) utiliza aproximações externas do poliedro, dadas por subconjuntos das facetas de $\mathcal{F}$. Ele pode então ser visto como um algoritmo de "geração de restrições", assim como Dantzig-Wolfe pode ser visto como um "gerador de colunas". Para o poliedro $\mathcal{F}$ o número total de restrições é em geral alto, mesmo o subconjunto de restrições que obtivemos em (3.40); basta ver que no grafo completo qualquer corte é minimal, e o número de cortes é da ordem de $2^{n}$. Conhecendo-se então o conjunto de todas as facetas, poderíamos procurar um critério para a geração dos cortes na ordem necessária para Falk-Hoffman. 


\section{Referências Bibliográficas}

[Ahuja, Magnanti \& Orlin 93]

[Bazaraa \& Jarvis 77]

[Benson 85]

[Bertsekas \& Tsitsiklis 89]

[Bezerra 84]

[Billera \& Sturmfels 92]

[Bretthauer 94]

[Dantzig 63]

[Dantzig \& Wolfe 60]

[Emelichev, Kovalev \& Kravtsov 84]
AHUJA, R. K.; MAGNANTI, T. L.; ORLIN, J. B.; Network Flows: Theory, Algoriths and Applications. Prentice-Hall, 1993.

BAZARAA, M. S.; JARVIS, J. J; Linear Programming and Network Flows. John Wiley \& Sons, 1977.

BENSON, H. P.; A Finite Algorithm for Concave Minimization Over a Polyhedron. Naval Research Logistics Quarterly, 32:165-177, 1985.

BERTSEKAS, D. P.; TSITSIKLIS, J. N.; Parallel and Distributed Computation - Numerical Methods. Prentice Hall, 1989.

BEZERRA, J. R. M.; Sobre problemas de otimização de redes de computadores. São Paulo, 1984. 233p. Dissertação (Mestrado) - IME-USP.

BILLERA, L. J.; STURMFELS, B.; Fiber Polytopes. Annals of Mathematics, 135:527-549, 1992.

BRETTHaUeR, K. M.; A Penalty for Concave Minimization Derived from the Tuy Cutting Plane. Naval Research Logistics, 41:455-463, 1994.

DANTZIG, G. B.; Linear Programming and Extensions. Princeton University Press, 1963.

DANTZIG, G. B.; WOLFE, P.; Decomposition Principle for Linear Programs. Operations Research, 8(1):101-111, 1960.

EMELICHEV, V. A.; KOVALEV, M. M.; KRAVTSOV, M. K.; Polytopes, Graphs and Optimization. Cambridge University Press, 1984. 
[Falk \& Hoffman 76]

[Ferreira \& Wakabayashi 96]

[Frata, Gerla \& Kleinrock 73]

[Ford \& Fulkerson 62]

[Geoffrion 70]

[Geoffrion 71]

[Gerla 73]

[Hoffman 81]

[Horst, Pardalos \& Thoai 95]

[Horst \& Thoai 89]

[Horst, Thoai \& Benson 91]

[Hu 63]

[Humes 78]
FALK, J. E.; HOFFMAN, K. R.; A Successive Underestimation Method for Concave Minimization Problems. Math. Of Operations Research, 1(3):251-259, 1976.

FERREIRA, C. E.; WAKABAYASHI, Y.; Combinatória Poliédrica e Planos-de-Corte Faciais. In.: 10a Escola de Computação, Campinas, 1996.

FRATTA, L.; GERLA, M.; KLEINROCK, L.; The flow deviation method: an approach to store-and-forward communication network design. Network, 3(2):97-133, 1973.

FORD, L. R.; FULKERSON, D. R.; Flows in Networks. Princeton University Press, 1962.

GEOFFRION, A. M.; Elements of large-scale mathematical programming. Management Science, 16(11):652-691, 1970.

GEOFFRION, A. M.; Duality in nonlinear programming: a simplified applications-oriented development. SIAM Review, 13(1):1-37, 1971.

GERLA, M.; The Design of Store-and-forward Networks for Computer Networks. Tese (doutorado), University of California, Los Angeles, 1973.

HOFFMAN, K. L.; A Method for Globally Minimizing Concave Functions Over Convex Sets. Math. Programming, 20:22-32, 1981.

HORST, R.; PARDALOS, P. M.; THOAI, N. V; Introduction to Global Optimization. Kluwer Academic Press, 1995.

HORST, R.; THOAI, N. V.; Modification, Implementation and Comparison of Three Algorithms for Globally Solving Linearly Constrained Concave Minimization Problems. Computing, 42:271-289, 1989.

HORST, R.; THOAI, N. V.; BENSON, H. P.; Concave Minimization via Conical Partitions and Polyhedral Outer Approximation. Math. Programming, 50:259-274, 1991.

HU, T. C.; Multi-commodity Flows. Operations Research, 11(3):344-360, 1963.

HUMES JR., C.; Problemas de grande porte em programação matemática. In: REUNIÃO DE MATEMÁTICA APLICADA, 1, Rio de Janeiro, 1978, 80p. Atas. IBM do Brasil. 
[Humes 88]

[Humes 89]

[Humes 91]

[Humes 92]

[Humes \& Humes 86]

[Humes \& Humes 91]

[Humes \& Ruggiero 84]

[Jackson 57]

[Kleinrock 76]

[Lin 76]

[Lomonosov 85]

[Luenberger 73]
HUMES JR., C.; Tópicos de otimização e redes de computadores. São Paulo, 1988, 109p. Tese (Livre-Docência) IME - Universidade de São Paulo.

HUMES JR., C.; Método de Designação de Fluxos e Capacidades: Versão Finita. Relatório Técnico do IME-USP, Dez/1989.

HUMES JR., C.; Some Results on Outer Linearizations in the Presence of Concavity. Pesquisa Operacional, 11(1):1-9, 1991.

HUMES JR., C.; A projection-feasible directions method for the continuous capacity and flow assignment. Matemática Aplicada e Computacional, 11(2), 1992. Birkhaueser-Boston.

HUMES JR., C.; HUMES, A. F. P. C.; Programação Linear - Um Primeiro Curso. In: IX Congresso Nacional de Matemática Aplicada e Computacional - SBMAC, Brasília, 1986.

HUMES, A. F. P. C.; HUMES JR., C.; A note on some results on outer linearizations in the presence of concavity. Pesquisa Operacional, 11(2):75-78, 1991.

HUMES JR., C. e RUGGIERO, N.V.; A recursive design method for centralized computer networks. In: COTLE, R.W.; KELMENSON, M.L.; KORTE, B.; (eds.) Mathematical Programming. Amsterdam, North-Holland, 1984. p.185-194.

JACKSON, J. R. ; Network of Waiting Lines. Operations Research, 5(4): 518-521, 1957.

KLEINROCK, L.; Queuing Systems. Vols I \& II. New York, John Wiley, 1975-1976, 2v.

LIN, J. G.; Maximal Vectors and Multiobjective Optimization. In: Journal of Optimization Theory and Applications, 18(1):41-64, 1976.

LOMONOSOV, M. V.; Combinatorial Approaches to Multiflow Problems. In.:Discrete Applied Mathematics, Vol 11(1), 1985.

LUEMBERGER, D. G.; Introduction to Linear and Nonlinear Programming. Addison-Wesley, 1973. 
[Magnanti \& Wolsey 95]

[Mangasarian 69]

[Pardalos \& Rosen 86]

[Pardalos \& Rosen 87]

[Rosen 83]

[Tanenbaum 89]

[Tuĭ 64]

[Tuй, Thieu \& Thai 85]

[White 93]

[Zangwill 68]

[Ziegler 95]

[Zwart 74]
MAGNANTI, T. L.; WOLSEY, L. A.; Optimal Trees. In: Handbooks in OR \& MS, Vol 7, Ch. 9. Elsevier Science B. V., 1995.

MANGASARIAN, O. L. ; Nonlinear Programming, McGraw-Hill, New York, 1969.

PARDALOS, P. M.; ROSEN, J. B.; Methods for Global Concave Minimization: A Bibliographic Survey. SIAM Review, 28(3):367-379, 1986.

PARDALOS, P. M.; ROSEN, J. B.; Constrained Global Optimization: Algorithms and Applications. Lecture Notes in Computer Science, 268. Springer-Verlag Berlin, 1987.

ROSEN, J. B.; Global Minimization of a Linearly Constrained Concave Function by Partition of Feasible Domain. Mathematics of Operations Research, 8(2):215-230, 1983.

TANENBAUM, A. S. ; Computer Networks. 2nd ed. Prentice-Hall, 1989.

TUĬ, H.; Concave Programming Under Linear Constraints. Soviet Math. Dokl., 5:1437-1440, 1964.

TUI, H.; THIEU, T. V.; THAI, NG. Q.; A Conical Algorithm for Globally Minimizing a Concave Function Over a Closed Convex Set. Mathematics of Operations Research, 10(3):498-514, 1985.

WHITE, D. J.; Extension of the Frank-Wolfe Algorithm to Concave Nondifferentiable Objective Functions. JOTA, 78(2):283-301, 1993.

ZANGWILL, W. I.; Minimum Concave Cost Flows in Certain Networks. Management Science, 14(7):429-450, 1968.

ZIEGLER, G. M.; Lectures on Polytopes. Springer-Verlag, 1995.

ZWART, P. B.; Global Maximization of a Convex Function with Linear Inequality Constraints. Operations Research, 22:602-609, 1974. 